University of Louisville

ThinkIR: The University of Louisville's Institutional Repository

Electronic Theses and Dissertations

$12-2011$

\title{
Conformational dynamics leading to activation of the transglutaminase factor XIII.
}

Richard Tatum Woofter

University of Louisville

Follow this and additional works at: https://ir.library.louisville.edu/etd

\section{Recommended Citation}

Woofter, Richard Tatum, "Conformational dynamics leading to activation of the transglutaminase factor XIII." (2011). Electronic Theses and Dissertations. Paper 1591.

https://doi.org/10.18297/etd/1591

This Doctoral Dissertation is brought to you for free and open access by ThinkIR: The University of Louisville's Institutional Repository. It has been accepted for inclusion in Electronic Theses and Dissertations by an authorized administrator of ThinkIR: The University of Louisville's Institutional Repository. This title appears here courtesy of the author, who has retained all other copyrights. For more information, please contact thinkir@louisville.edu. 


\title{
CONFORMATIONAL DYNAMICS LEADING TO ACTIVATION OF THE
}

TRANSGLUTAMINASE FACTOR XIII

\author{
By \\ Richard Tatum Woofter II \\ B.S., Biochemistry, College of Charleston, 2000 \\ B.S., Chemistry, College of Charleston, 2001
}

\author{
A Dissertation \\ Submitted to the Faculty of the \\ College of Arts and Sciences of the University of Louisville \\ In Partial Fulfillment of the Requirements \\ For the Degree of \\ Doctor of Philosophy \\ Department of Chemistry \\ University of Louisville \\ Louisville, Ky \\ December 2011
}


Copyright 2011 by Richard Tatum Woofter II

All rights reserved 
CONFORMATIONAL DYNAMICS LEADING TO ACTIVATION OF THE TRANSGLUTAMINASE FACTOR XIII

By

Richard Tatum Woofter II

B.S., Biochemistry, College of Charleston, 2000

B.S., Chemistry, College of Charleston, 2001

Dissertation approved on

December 16, 2011

by the following Dissertation Committee:

Dissertation Director, Muriel C Maurer 


\section{DEDICATION}

This dissertation is dedicated to all the love and support of my family. First and foremost, graduate school would not have been possible without the loving support of my beautiful wife Molly. I am truly fortunate to have fallen in love with my best friend. Thank you for all of your assistance/love/support throughout this journey.

Denise, I did not know my own capacity to love until you were brought into this world in 2005. You are a mere 6 years old and I admire your wisdom and drive to learn. I look forward to watching you become a "veterinarian Princess."

Isaac, where do I start? You have brought nothing but smiles since you were born in 2007. You have an amazing ability to make people laugh. You are now 4 years old and your imagination is unstoppable.

Norah, you came into this family ready to play. You joined our family in 2009 and there is nothing that slows you down. Your manners and thoughtfulness are second to none.

I love you guys... You are my Life.

Lastly, I have to thank the folks that managed to keep me alive from $0-18$ years of age. I like to think that the genetics of an English teacher mixed with a computer programmer/machinist make for the best scientists. Thanks for all the help throughout the years from diagramming sentences to coaching soccer/baseball/... to just being parents. 


\section{ACKNOWLEDGEMENTS}

There are those people that come into your life and make an impression that is not easily forgotten. Dr. Muriel Maurer is one of those people. Her love and dedication to science is something to be admired. I am personally indebted to her for her patience in training me and guiding me over the past 4 years. I look forward to maintaining close contact with her as I feel she is not just my graduate advisor, but also my friend.

The NMR spectra presented in this dissertation would not have been possible without Dr. Richard Wittebort's assistance. He was extremely patient with Dr. Maurer and I as we were learning how to effectively use NMRPIPE and SPARKY. I would also like to thank the rest of my committee Dr. Mueller, Dr. D'Souza, and Dr. Zhang. I am indebted to you for your comments throughout the proposal and research process.

In the early days, I had the wonderful opportunity to learn HDX under Dr. Mike Sabo, one of Dr. Maurer's previous students. In the few months that I was able to interact with Mike I gained the utmost respect for his knowledge of not only HDX coupled with MALDI-TOF-MS, but also NMR. He was truly a gift to have in the lab.

The TG2 project would have not been possible without the wonderful assistance of Dr. Michael Merchant and Daniel Wilkey in the Clinical Proteomics 
Center at the University of Louisville. The use of their AB 4700 MALDI-TOF-MS allowed for efficient identification of all peptic peptides utilized in the TG2 study and increased our sequence coverage for FXIII-A.

My colleagues in the lab, Madhavi A. Jadhav, Marina Malovichko and Prakash G. Doiphode, I have enjoyed learning with you. As the only American student in the lab I was felt fortunate to have the opportunity to learn more about the Russian and Indian culture. Some of my favorite memeories from graduate school are working with you guys in the lab.

I need to thank all my CGSA colleagues. Through the organization of graduate student events, we have had many priceless opportunities. Dr. Mike Nantz, "Ricky Bobby" would like to thank you for being a cornerstone in my early years, Thanks you. Dr. Rich, Thank you for all the wonderful memories from the GEMS program. The experience of teaching $7^{\text {th }}$ grade science is irreplaceable. Lastly, the Chemistry Department at the University of Louisville now holds a special place in my heart and I look forward to continuing my relationship with the Department of Chemistry faculty and staff. 


\section{ABSTRACT \\ CONFORMATIONAL DYNAMICS LEADING TO ACTIVATION OF THE TRANSGLUTAMINASE FACTOR XIII}

Richard Tatum Woofter II

16 December 2011

One of the last events that occurs during blood coagulation, a process taken for granted on a daily basis, involves Factor XIII (FXIII) cross-linking fibrin monomers to form an insoluble clot. In plasma, FXIII- $A_{2}$ is not active and exists as the heterotetramer FXIII- $A_{2} B_{2}$. Through the utilization of hydrogen deuterium exchange (HDX) coupled with Matrix Assisted Laser Desorption ionization - Time of Flight - Mass Spectrometry (MALDI-TOF-MS), it was determined that FXIII-A $A_{2}$ becomes nearly uniformly protected when bound to FXIII- $B_{2}$ and the FXIII-A $A_{2} \beta$-barrels play a major role in heterotetramer formation.

After dissociation from FXIII-B $B_{2}$ FXIII- $A_{2}$ has the ability to become activated in the presence of $\mathrm{Ca}^{2+}$. The regions/residues of $\mathrm{FXIII-A} \mathrm{Ca}_{2}^{2+}$ affects during activation were identified using HDX. It is debated whether FXIII-A undergoes an open conformation during activation. Transglutaminase 2 (TG2) has been observed crystallographically in an open conformation. HDX was utilized to compare the conformational dynamics of Transglutaminase 2 in solution to that of FXIII-A. The increase in exposure between the catalytic core and $\beta$-barrels of TG2 yields evidence of an open conformation. A structural 
comparison of FXIII-A $A_{2}$ and TG2 identified steric hinderance within the $A_{2}$ dimer that could thwart a similar conformational change.

Once activated physiologically, FXIII-A is solely responsible for forming the cross-links between fibrin monomers. The $\alpha \mathrm{C}(233-425)$ region of fibrin contains three reactive GIn residues and acts as a substrate for FXIII. Fibrin $a \mathrm{C}$ $(233-425)$ was expressed and its structure investigated via ${ }^{15} \mathrm{~N}-\mathrm{HSQC}$ when in solution with FXIII-A

The integral role of FXIII-A $A_{2}$ in the coagulation cascade leads to a dire need for investigating its conformational dynamics during activation. The research herein provides a stronger knowledge of FXIII-A $A_{2}$ structural changes during activation and outlines FXIII- $A_{2}$ interactions with $B_{2}$ and the fibrin $a C$ domain. This progress in understanding FXIII-A $A_{2}$ dynamics could lead to improved treatments for excessive bleeding and thrombosis. 
TABLE OF CONTENTS

Page

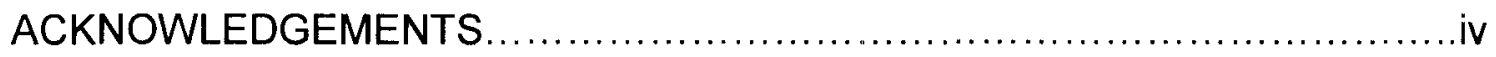

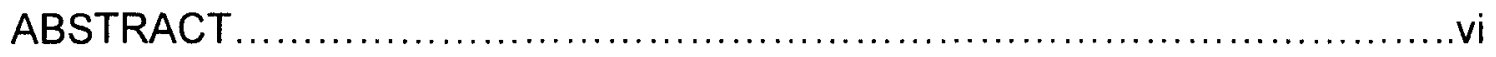

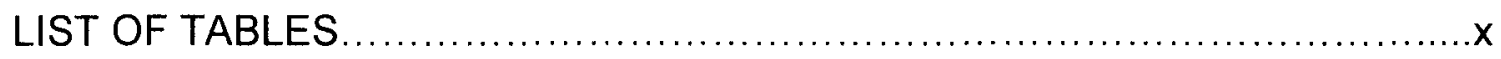

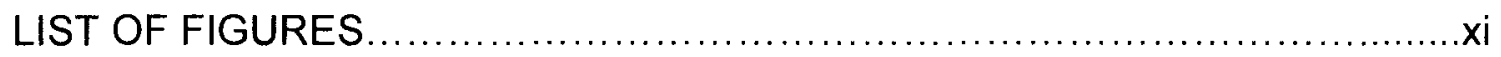

CHAPTER

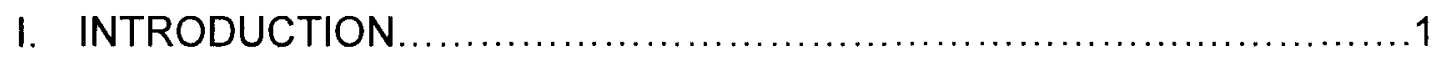

II. ROLE OF CALCIUM IN THE CONFORMATIONAL DYNAMICS OF

FACTOR XIII ACTIVATION EXAMINED BY

HYDROGEN/DEUTERIUM EXCHANGE COUPLED WITH MALDI-

TOF MS

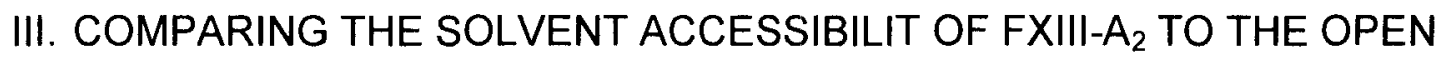
AND CLOSED FORM OF TRANSGLUTAMINASE 2.

IV. UTILIZATION OF HDX TECHNOLOGY TO ELUCIDATE THE FXIII $A_{2}$ RESIDUES CRUCIAL FOR HETEROTETRAMER FORMATION WITH

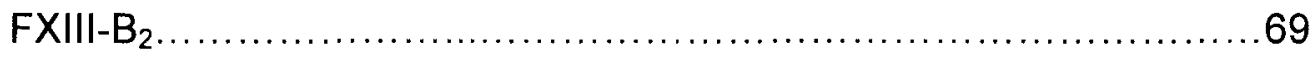

V. DEVELOPMENT OF A FIBRINOGEN ALPHA-C EXPRESSION SYSTEM UTILIZING MINIMAL MEDIA AND SUBSEQUENT ALPHAC STRUCTURAL ANALYSIS VIA NMR. 
VI. CONCLUSIONS AND FUTURE DIRECTIONS

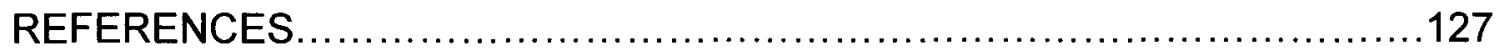

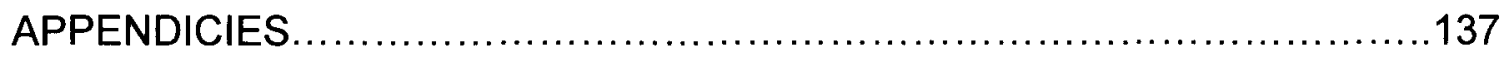

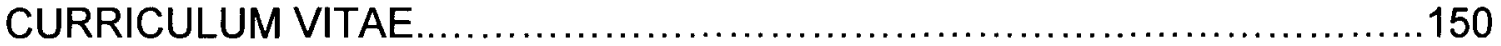




\section{LIST OF TABLES}

Table 1: Changes in Percent Deuteration: FXIII with $1 \mathrm{mM}$ Metal Relative to

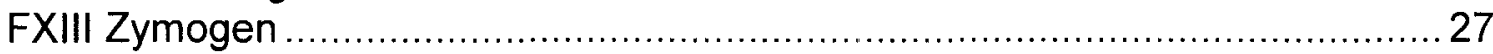

Table 2: Changes in Percent Deuteration: Nonproteolytically Activated FXIII Relative to FXIII Zymogen ....................................................................... 31

Table 3: Changes in Percent Deuteration: Active TG2 and Inhibited TG2 relative

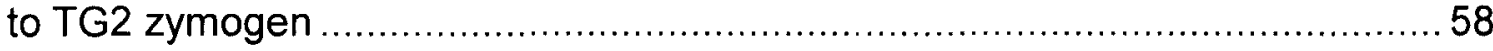

Table 4: Changes in Percent Deuteration: $F X I I I-A_{2} B_{2}$ relative to $F X I I I-A_{2}$ zymogen 80

Table 5: Components of $1 \mathrm{~L} 5 \times \mathrm{M} 9$ salts and $1 \times$ concentration.......................98

Table 6: Recipe for M9 minimal media. .................................................... 99

Table 7: The protease inhibitors utilized during purification of recombinant protein 


\section{LIST OF FIGURES}

Figure 1: The conventional $Y$-shaped cascade model of coagulation. .............. 2

Figure 2: The Cell-Based Model of Hemostasis. ........................................... 4

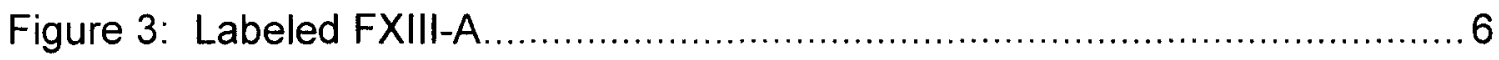

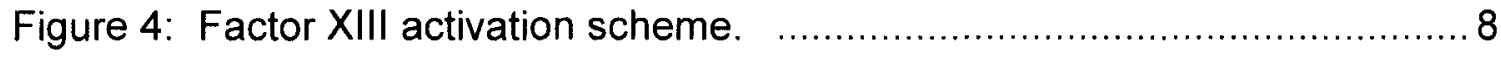

Figure 5: Schematic describing the processes involved in hydrogen-deuterium exchange coupled with MALDI-TOF-MS ................................................ 12

Figure 6: The FXIII A zymogen 2.1 $\AA$ crystal structure ............................... 17

Figure 7: FXIII peptic peptides observed by MALDI-MS …............................2 24

Figure 8: The number of deuterons incorporated for FXIII under the following conditions: FXIII zymogen, $1 \mathrm{mM} \mathrm{Ca}^{2+}, 1 \mathrm{mM} \mathrm{Mg}^{2+}, 1 \mathrm{mM} \mathrm{Ba}^{2+}$ and $1 \mathrm{mM} \mathrm{Cu}^{2+} 26$

Figure 9: TGase activity of nonproteolytically activated FXIII. .........................30

Figure 10: The number of deuterons incorporated for activated FXIII. ............. 33

Figure 11: Illustration of the regions within the catalytic core and the $\mathrm{Ca}^{2+}$ binding

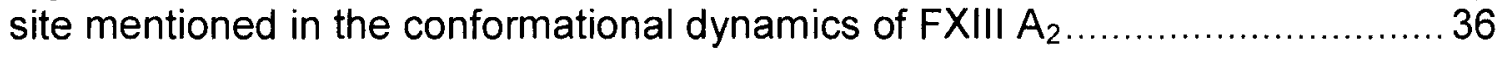

Figure 12: An illustration of all peptic peptides which yield sequence coverage along the dimer interface.

Figure 13: Illustration of the substrate recognition regions and dimer interface mentioned in the conformational dynamics of FXIII $A_{2}$

Figure 14: Illustration of the activation peptide and its intimate contact with the opposing FXIII-A monomer

Figure 15: Structure of GDP-Bound and Inhibitor-Bound TG2 and FXIII-A 2 Dimer.

Figure 16: K9 DON Inhibition of FXIIla. 
Figure 17: TG2 peptic peptides observed by MALDI-TOF-MS 55

Figure 18: A plot of $\%$ inhibition vs the log of TG-DON concentration. 56

Figure 19: The number of deuterons incorporated at $10 \mathrm{~min}$ for TG2 under the following conditions: zymogenic TG2, $2 \mathrm{mM} \mathrm{Ca}^{2+}, 2 \mathrm{mM} \mathrm{Ca}^{2+}$ with TG-DON. .. 59

Figure 20: Overlay comparison of the TG2 $\beta$-Sandwich domain in the open and closed conformations. 62

Figure 21: Overlay comparison of TG2 in the open and closed conformations. 63

Figure 22: Illustration of the intimate connection between the catalytic triad and peptides $342-354$ and $370-378$. 64

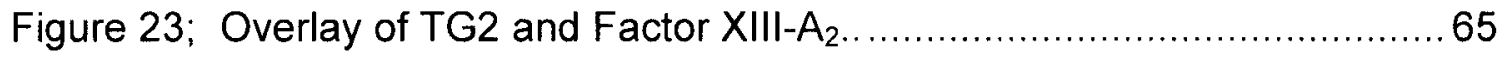

Figure 24: Overlay of FXIII-A $A_{2}$ and the open conformation of TG2 ...............66

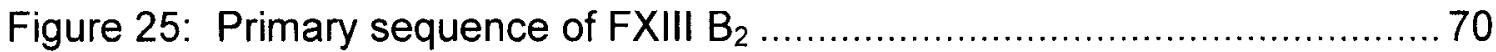

Figure 26: Cartoon model of FXIII B homodimer.......................................... 71

Figure 27: Representative spectra for FXIII-A2, FXIII-B ${ }_{2}$ and FXIII- $A_{2} B_{2}$ following

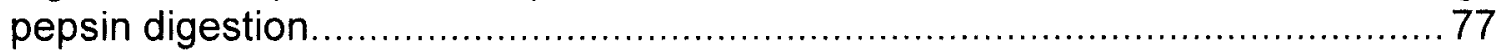

Figure 28: FXIII peptic peptides observed by MALDI-TOF-MS ....................... 78

Figure 29: Native PAGE illustrating the formation of FXIII- $A_{2} B_{2}$. Molecular

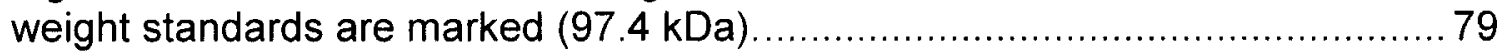

Figure 30: The number of deuterons incorporated for $F X I I I-A_{2}$ and $F X I I I-A_{2} B_{2} \ldots 81$

Figure 31: Illustration of FXIII-A $\mathrm{A}_{2} \mathrm{~B}_{2} \mathrm{HDX}$ sequence coverage. ...................... 84

Figure 32: Representative FXIII-A $\mathrm{A}_{2}$ HDX Spectra..................................... 85

Figure 33: $\beta 2$-glycoprotein I - Representative sushi domain........................8 87

Figure 34: Schematic of fibrionogen and the dissociation of the $\mathrm{aC}$ domain when

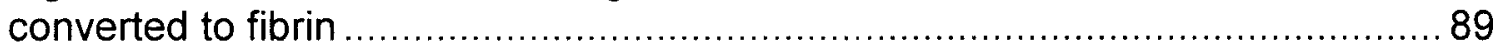

Figure 35: aC Domain (233-425) PAGE confirmation...................................... 91

Figure 36: Representative tripeptide displaying the magnetization transfer during

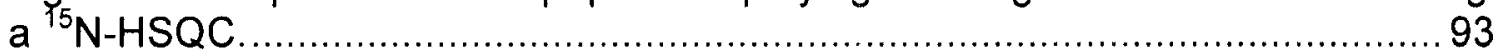


Figure 37: Visualization of aC (233-425) purification following expression in BL21 Gold E. coli cells.

Figure 38: Map of pGEX-6P-1 vector.

Figure 39: Alignment of $3^{\prime}$ primer sequence data and $\alpha C(233-425)$ gene sequence

Figure 40: Representative GST specific Western Blot.

Figure 41: Representative SDS-PAGE and western blot confirming proper aC $(233-425)$ purification and GST cleavage

Figure 42: ${ }^{15} \mathrm{~N}-\mathrm{HSQC}$ spectra of ${ }^{15} \mathrm{~N}-\alpha \mathrm{C}(233-425)$ conducted on an $800 \mathrm{MHz}$ Varian NMR at $25^{\circ} \mathrm{C}$.

Figure 43: ${ }^{15} \mathrm{~N}-\mathrm{HSQC}$ spectra of ${ }^{15} \mathrm{~N}-\mathrm{aC}(233-425)$ in the presence of $\mathrm{FXIII-A_{2 }}$ conducted at $25^{\circ} \mathrm{C}$ on an $800 \mathrm{MHz}$ Varian NMR.

Figure 44: Theoretical E. coli growth curve ............................................. 115

Figure 45: Representative growth curves for BL21 gold (DE3) cells grown in LB broth and minimal media.

Figure 46: ${ }^{15} \mathrm{~N}-\mathrm{HSQC}$ spectra of ${ }^{15} \mathrm{~N}-\alpha \mathrm{C}(233-425)$ overlaid on the spectra for ${ }^{15} \mathrm{~N}-a \mathrm{C}(233-425)$ in the presence of $\mathrm{FXIII-A_{2 }}$. 


\section{CHAPTER I}

\section{INTRODUCTION}

\section{Blood Coagulation Overview}

The highway system that transports nutrients to all cells within the body and aids in eliminating cellular waste is referred to as the circulatory system. The circulatory system involves many organ systems working in harmony with one another. The organ that acts as the carrier for all the nutrients, amino acids, hormones and cellular waste is blood (1). If there is a breach in the vasculature there needs to be processes in place which quickly and efficiently close the breach while not allowing for any disturbance in the flow of blood (nutrients) to cells throughout the body. Haemostasis ('hemo' = blood + 'stasis' = stagnation), the process of stopping blood flow in damaged tissue, starts with vasoconstriction to slow the flow of blood to the damaged area. This physiological process can also be assisted by external pressure on wounded tissue. Following vasoconstriction, platelets are recruited to the damaged tissue to form a plug. As the platelets aggregate, tissue factors signal the coagulation cascade which ultimately concludes in a soluble fibrin clot being crosslinked by factor XIII (FXIII) to form an insoluble clot (1). 
Historically it was believed

that coagulation was initiated after

an injury when blood was exposed

to air. It was not until the mid- $19^{\text {th }}$

century before it was proposed that

a specific component in the blood

initiated coagulation (2). Nearly a

century later in 1964, the "Cascade"

or "Waterfall" model was released

by two independent groups (3-4).

These models were developed

under the premise of defining the

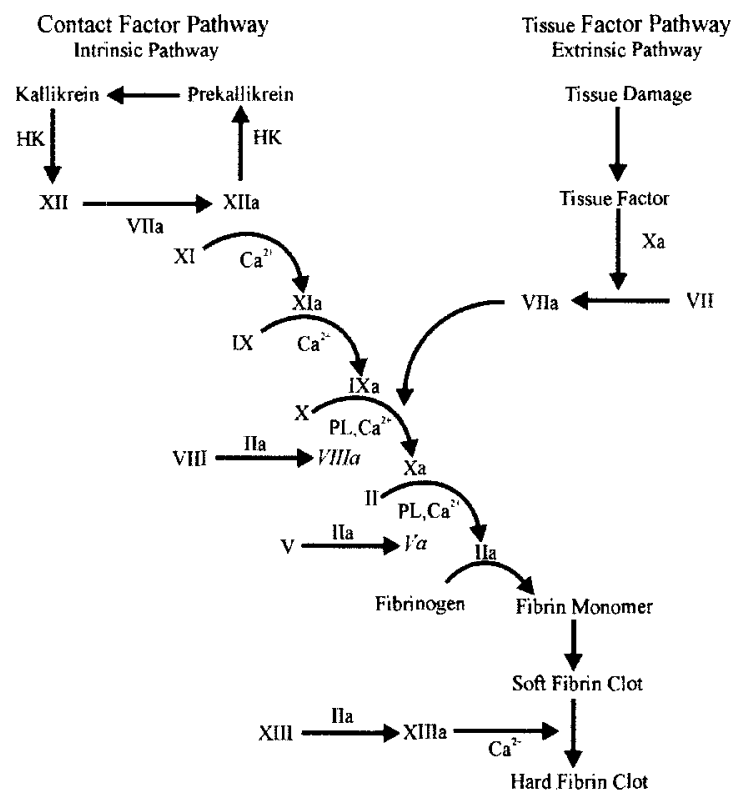

Figure 1: The conventional Y-shaped cascade model of coagulation. This model initiates via the Intrinsic (contact factor) pathway or the extrinsic (tissue factor) pathway. This diagram was adapted from a poster distributed by Enzyme Research Laboratories, South Bend, IN.

identity and function of the procoagulant enzymes. The early models involved the familiar Y-shaped scheme (Figure 1 or for more detail Appendix A) where the "intrinsic" and "extrinsic" pathways converged in a common pathway with the production of Factor Xa After several decades of discoveries, questions started to arise concerning the independent nature of the intrinsic and extrinsic pathways. One such question being, why do patients with a deficiency of FVIII and FIX (see appendix B for coagulation enzyme abbreviations) in the "intrinsic" pathway contract hemophilia A and B respectively when they still have an intact "extrinsic" pathway(5). Hoffman and Monroe have since worked to develop a model in which coagulation is mediated by the cell surfaces where they occur. They propose a model that incorporates three overlapping stages: 1- initiation, 
occurring on tissue factor baring cells; 2-amplification, occurs when platelets and cofactors become activated prior to thrombin production; and 3- propagation, where large amounts of thrombin are generated on the platelet surface (6). Initiation

Tissue factor (TF) is an integral membrane protein that plays the role of initiator in coagulation in vivo. As a membrane bound protein, TF remains localized to the cell in which it was synthesized and these cells are generally located outside the vasculature (7). Once there is a breach in the vessel, blood will come into contact with the TF-bearing extravascular cells. FVII is easily activated by coagulation and non-coagulation proteases and rapidly binds to cellular TF. The FVIla-TF complex then goes on to further activate more FVII as well as both FX and FIX. The FX that is activated by the FVIIA-TF complex is quickly degraded if it leaves the cell surface, but when it remains within the confines of the forming thrombus, it can combine with FVa to produce thrombin which will aid in activating platelets and FVIII during amplification (8).

\section{Amplification}

The next step is to amplify the coagulation response that was just initiated. The damage to the endothelium that initiated the response also allows platelets to come in contact with TF-bearing cells. The thrombin that was produced during the last stages of initiation is then utilized to enhance platelet adhesion and activation (9). Thrombin also assists in preparing for procoagulant activity by activating FV, FVIII, and FXI (8). 


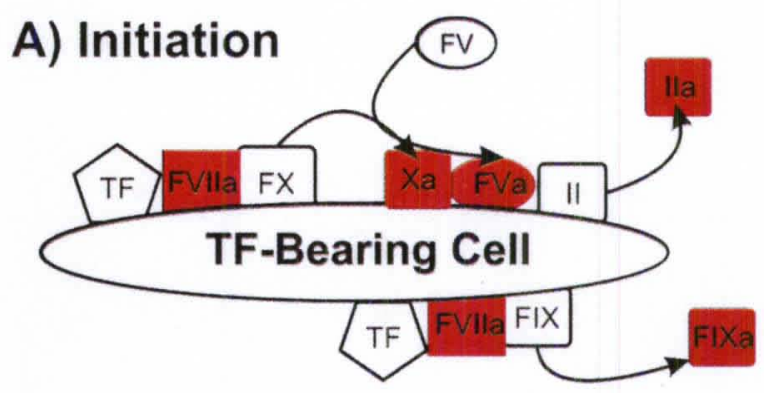

B) Amplification

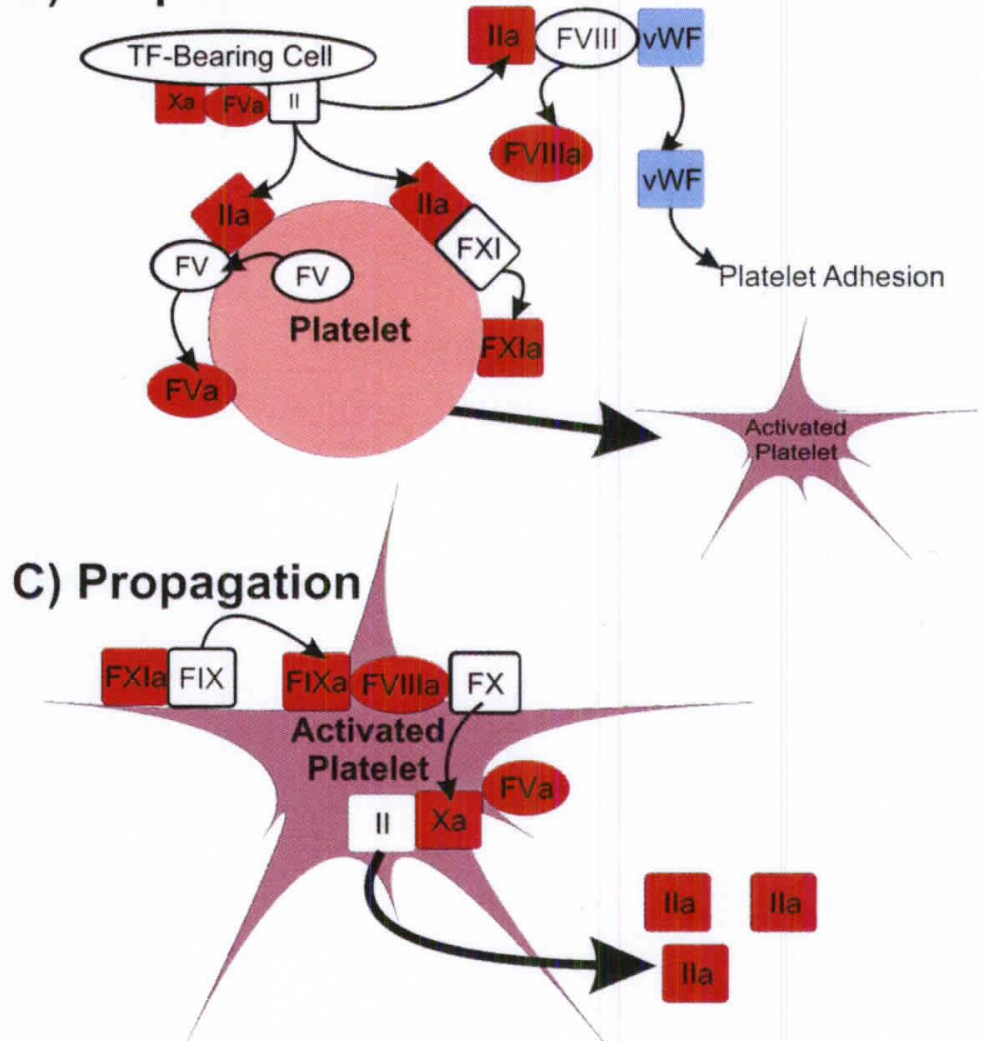

Figure 2: The Cell-Based Model of Hemostasis. In the cell based model, several cell surfaces are utilized in during the series of events that lead to a fibrin clot. (A) During the Initiation phase TF-bearing cells become exposed to plasma after injury and FIXa and Ila are released and diffuse to the platelet surface. (B) During Amplification the Ila released on the TF-bearing surface serves to activate the platelets and FXI as well as release vWF yielding FVIIla. (C) During Propagation several of the enzymes that were activated earlier assemble on the surface of the activated platelet. FIXa/FVIla form the tenase which aid in FXa formation. $\mathrm{FXa/FVa}$ then form prothrombinase which results in the burst of Ila. Ila then goes on to cleave fibrinogen and activate FXIII which crosslinks the fibrin to form an insoluble clot. Figure adapted from cell-based hemostasis models $(2,6)$.

Thrombin continues its action by activating platelets through the utilization of their protease-activated receptors (PAR) (10). FV is released from platelets and subsequently activated by thrombin. Some of the thrombin remains on the surface of the platelet and is available for cleaving other coagulation enzymes. von Willebrand factor (VWF)/FVIII circulate together and when bound to the 
platelet surface, thrombin activates the FVIII through the release of VWF (11). The amplification process has now produced activated platelets with activated cofactors such as FVa, FVIIla and FXla on their surfaces; therefore, propagation is ready to commence.

Propagation

The FIXa and FVIIla that were activated during initiation and propagation respectively are now poised to form the intrinsic tenase complex on the surface of activated platelets. Once FIXa diffuses from the TF-bearing cell to the platelet these complexes begin to form. In addition to FIXa diffusing from the neighboring TF-bearing cell, it can also be activated on the platelet surface by FXla (12). The tenase complexes then continue the coagulation processes by activating FX on the platelet surface where it is poised to complex with FVa. These FXa/FVa "prothrombinase" complexes convert II to lla generating a lla burst (6).

The lla generated during initiation, amplification and propagation is further utilized in clot stabilization. Fibrinogen is a $340 \mathrm{kDa}$ protein that consists of three chains ( $A \alpha, B \beta$ and $y)$ forming the homodimer $(A \alpha B \beta \gamma)_{2}$. Thrombin converts fibrinogen to fibrin through the cleavage of fibrinopeptides $A$ and $B$. Fibrin then non-covalently self associates in the formation of a soft clot (13). The soluble fibrin clot is then stabilized through $Y$-glutamyl- $\varepsilon$-lysinyl cross-links formed between the fibrin $\gamma-\gamma$ and $\gamma-\alpha$ chains by lla-activated FXIII (14). Thrombin activated FXIII further stabilizes and protects the growing fibrin network from lysis 
by cross-linking it to thrombin-activatable fibrinolysis inhibitor (TAFI) and $\alpha_{2}$ antiplasmin $\left(\alpha_{2} A P\right)(15-17)$.

The process of haemostasis has been broken down into three overlapping stages: Initiation, Amplification, and Propagation. Of the many enzymes and cofactors involved, the research presented here focuses FXIII. The use of techniques such as HDX coupled with MALDI-TOF-MS aim to shed light into the illusive FXIII conformational changes.

Transglutaminase Factor XIII

FXIII- $A_{2}$ has gained much

interest over the last couple

decades due to its presence in the

cytoplasm of several different cell

types: macrophages,

megakaryocytes, and connective tissue histiocytes to name a few (19-21). Factor XIII plays an

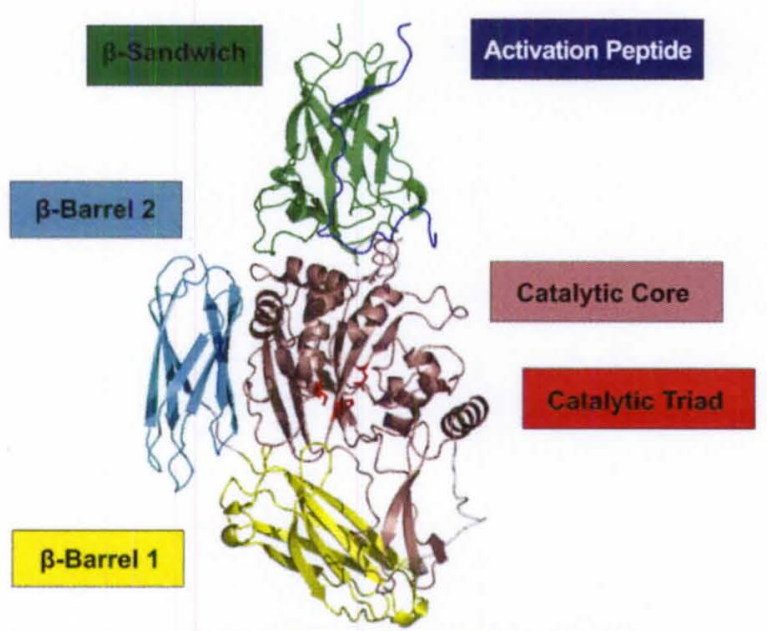

Figure 3: Labeled FXIII-A (1FIE) This figure was made using PyMol(18).

integral role in the coagulation cascade, and there is much need to investigate the conditions that affect activation/activity as well as the structural dynamics of the enzyme during activation. A better understanding of the structural changes around the active site of FXIII during activation, as well as how it interacts with the other enzymes and proteins involved in the coagulation cascade, could lead to more targeted therapeutic agents for treatment of excessive bleeding, thrombosis, and atherosclerosis. 
The action of Factor XIII was first reported in the early 1920s when Barkan and Gaspar observed that fibrin clots formed in the presence of $\mathrm{Ca}^{2+}$ were insoluble in weak bases (22). Over 25 years later in a letter to Nature, Lorand reported that a protein was responsible for the formation of insoluble clots because the "fibrin stabilizing factor" was thermolabile, nondialyzable and required $\mathrm{Ca}^{2+}(23)$. Then after the acknowledgement of fibrin stabilizing factor as a clotting factor in 1963 by the International Committee on Blood Clotting Factors, it became factor XIII (24). It soon became obvious that FXIII was essential for stabilizing the non-covalent soft clots formed in blood coagulation after thrombin cleaves the A and B fibrinopeptides from fibrinogen.

Factor XIII is a protransglutaminase in plasma with a tetrameric structure consisting of two $A$ subunits and two $B$ subunits $\left(F X I I I-A_{2} B_{2}\right)$. The details of FXIII-B will be further discussed in chapter IV, but briefly, all FXIII-A in plasma is found bound to FXIII-B as a FXIII- $\mathrm{A}_{2} \mathrm{~B}_{2}$ heterotetramer with an appearant $\mathrm{Kd}$ of $25 \mathrm{nM}(25)$. In platelets, FXIII-A $A_{2}$ is found without the $B_{2}$ dimer and is referred to as cellular FXIII (26). The $\sim 83 \mathrm{kDa}$ factor XIII-A has been successfully expressed and the recombinant form, $\mathrm{rFXIII}$, has been subsequently crystallized (27). The crystal structure illustrates the well-defined and sequentially folded domains: the activation peptide (1-37), the $\beta$-sandwich (38-184), the catalytic core (185-515), $\beta$-barrel 1 (516-628), and $\beta$-barrel 2 (629-730) (Figure 3). 


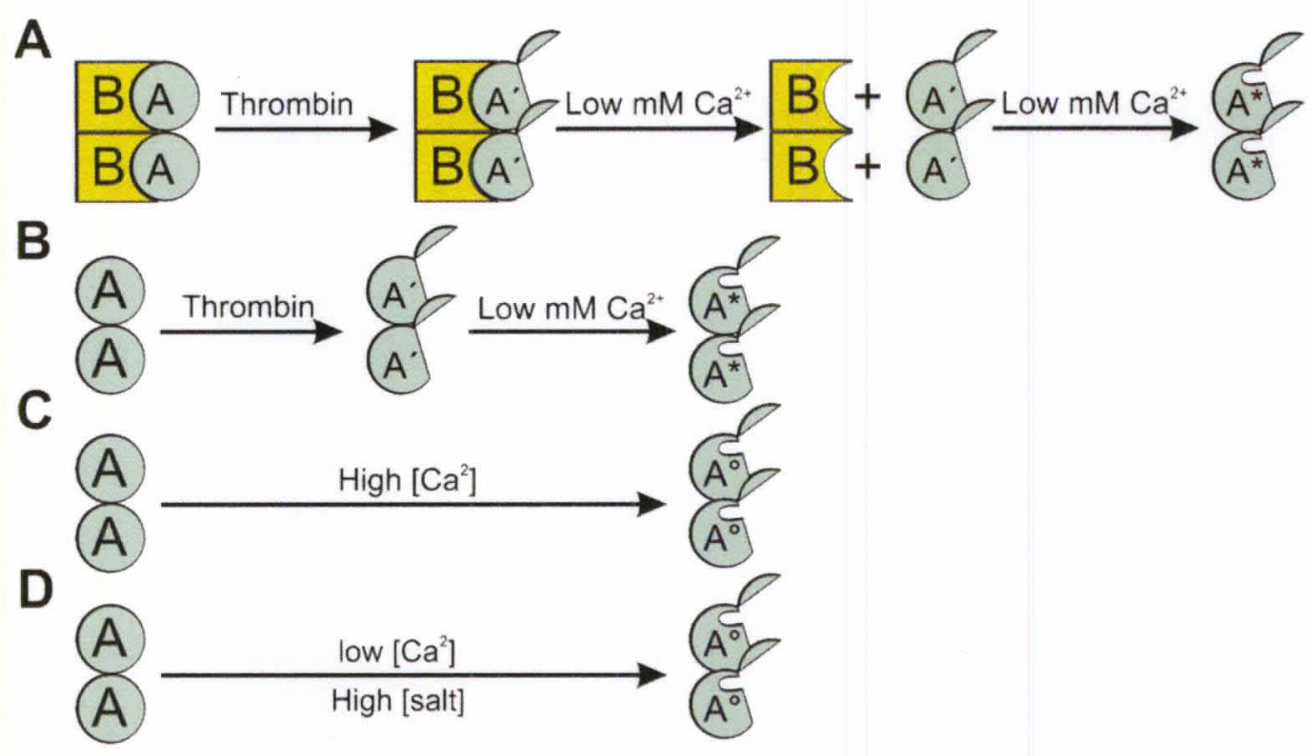

Figure 4: Factor XIII activation scheme. A) Plasma FXIII activation. Intact FXIII- $\mathrm{A}_{2} \mathrm{~B}_{2}$, thrombin cleaved FXIII-A', $\mathrm{Ca}^{2+}$ induced dissociation of FXIII-A $A_{2}$ and FXIII-B $B_{2}$ yeilding active FXIII-A* (FXIIIalla $)$. B - D) Cellular FXIII-A $A_{2}$ activation. B) Thrombin proteolytic activated FXIII-A $A_{2}$, thrombin cleaved FXIII-A' and fully active FXIII-A ( $\mathrm{FXIII}^{\text {Ila }}$ ) in the presence of low $\mathrm{MM} \mathrm{Ca}^{2+}$. C) FXIII-A $\mathrm{A}_{2}$ nonproteolytic activation in the

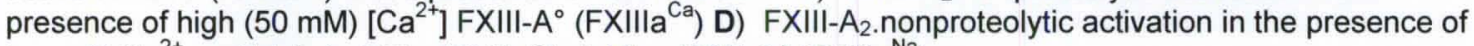
low $\mathrm{mM} \mathrm{Ca}^{2+}$ and high $(>150 \mathrm{mM}) \mathrm{NaCl}$ yielding $\mathrm{FXIII}^{\circ} \mathrm{A}^{\circ}\left(\mathrm{FXIIIa}^{\mathrm{Na}}\right)$.

When FXIII is activated physiologically (Figure 4), thrombin (Ila) cleaves the N-terminal activation peptide (residues 1-37). Following activation peptide cleavage, both low $\mathrm{mM} \mathrm{Ca}^{2+}$ and the C-terminal portion of the fibrin $\alpha$-chain assists in the dissociation of the two B subunits (28-32). Once the FXIII-A is free from FXIII-B $B_{2}$ it is active (FXIIIIIIa). Factor XIII exists as a dimer and the active site consists of a catalytic triad $\left(\mathrm{C}^{314}, \mathrm{H}^{373}\right.$ and $\left.\mathrm{D}^{396}\right)$. The catalytic $\mathrm{C} 314$ has an apparent $p K_{a}$ of 6.0 , similar to that of a histidine imidazole (33). The hydroxyl oxygen of Y560 forms a hydrogen bond with C314 (27). This H-bonding interaction is indicative of a thiolate - imidazolium pair (34). The catalytic sulfur anion is stabilized at physiological $\mathrm{pH}$ through the $\mathrm{H}$-bonding with $\mathrm{Y} 560$ and ionpair interactions with the $\mathrm{H} 373$ imidazolium $(27,34-35)$. 
Dimer formation is important for the stability of FXIII-A. The dimer interface covers $2280 \AA^{2}$ and there is a $10.3-14.4 \mathrm{kcal} / \mathrm{mol}$ decrease in its free energy upon dimerization (36). When in the presence of a suitable acyl-donor glutamine containing substrate, C314 of FXIIla forms a thioester bond and ammonia is released. The acyl-donor substrate is then covalently linked to a primary amine (lysine) acyl-acceptor forming $y$-glutamyl- $\varepsilon$-lysine cross-links between the a- and $y$-chains of fibrin which stabilizes the growing clot. In addition to clot stabilization, FXIII also assists in protecting the clot from plasmin degradation via crosslinks between the Aa-chain of fibrin and $\alpha_{2}$ antiplasmin(15, $17,37)$.

FXIII can also be non-proteolytically activated (Figure $4 \mathrm{C}$ and D) when the $\mathrm{Ca}^{2+}$ concentration is greater than $50 \mathrm{mM}\left(\mathrm{FXIIIa}^{\mathrm{Ca}}\right)$ (38-39). Under these non-physiological conditions the activation peptide is not cleaved and the enzyme shows activity. Another nonproteolytic activation mechanism has been demonstrated that is more physiologically relevant. When the factor XIII-A subunits are in the presence of $150 \mathrm{mM} \mathrm{Na}^{+}$with low $\mathrm{mM} \mathrm{Ca}^{2+}\left(\mathrm{FXIII}^{\mathrm{Na}}\right)$, albeit slow, transglutaminase activity is observed (40). When haemostasis concludes, one means that FXIII-A $A_{2}$ can be rendered inactive is through cleavage at the secondary cleavage site (K513-S514).

Hydorgen Deuterium Exchange (HDX) Coupled with Mass Spectrometry

The major theme underlying this research is establishing the conformational dynamics of FXIII-A in solution. The FXIII-A $A_{2}$ crystal structures provided to date for zymogen (27), FXIIIalla $(41)$, and FXIIla ${ }^{\mathrm{Ca}}$ (42) display no 
significant RMSD between the activated and unactivated forms. The active site is occluded in all crystal structures so an active conformation has yet to be observed. In an effort to gain a better understanding of FXIII-A during activation, HDX has been utilized in several studies (43-46). HDX has proved to be a worthy method of isolating structural changes to defined regions of the molecule

Proteins in solution are not static and in fact are naturally moving, flexing, and possibly even opening and closing. These movements termed, conformational dynamics, are difficult to monitor and impossible to see via crystallography. One benefit of HDX in the field of protein dynamics is the needed sample size. A typical HDX sample contains $12 \mu \mathrm{l}$ of $30-50 \mu \mathrm{M}$ protein whereas NMR would require approximately $350 \mu \mathrm{l}$ and preferably $>100 \mu \mathrm{M}$ protein. At pH 7.0 exposed protein backbone amide hydrogens exchange with $\mathrm{D}_{2} \mathrm{O}$ within $1-10$ seconds (47). Within the interior of the protein, exchange is limited because there is not only restricted access but also nearly all amide hydrogens are involved in hydrogen bonds. The $\mathrm{H}$-bonding protects the hydrogen from exchange (48). When running HDX at physiological $\mathrm{pH}$, basecatalyzed exchange is the dominant mechanism of hydrogen exchange. This occurs in the presence of a catalyst (hydroxide) and a new hydrogen (water) (47). 
The rate of hydrogen

exchange in an unfolded protein is $F_{H} \underset{k_{-1}}{\stackrel{k_{1}}{\rightleftharpoons}} U_{H} \underset{\mathrm{D}_{2} \mathrm{O}}{\stackrel{k_{2}}{\rightleftharpoons}} U_{D} \underset{k_{1}}{\stackrel{k_{-1}}{\rightleftharpoons}} F_{D}$ dependent upon several factors.

In Equation 1, $\mathrm{U}$ and $\mathrm{F}$ represent

Equation 1: Rate Constants for hydrogen-deuterium exchange where $F$ and $U$ represent folded and unfolded protein respectively (47).

the unfolded and folded protein respectively; therefore, hydrogen-deuterium exchange is not only dependent upon $\left(k_{2}\right)$, but also the $k_{1}$ and $k_{-1}$ (the unfolding and folding of the protein). The exchange kinetics are broken down into two pathways, EX1 and EX2 kinetics. When $k_{2} \gg k_{-1}$ EX1 kinetics ensue. Under EX1 kinetics, there is an unfolding event and the residues involved are exchanged prior to refolding. This is very rarely seen naturally, without denaturants. Therefore, EX2 kinetics typically prevail where the protein is quickly exchanging between two states and the refolding rate is faster than the exchange rate $\left(k_{-1}>>k_{2}\right)$. Once hydrogen-deuterium exchange occurs, the half-life for amides increases to over an hour by lowering the $\mathrm{pH}$ to between 2 and 3 at $0{ }^{\circ} \mathrm{C}$ (48). This low $\mathrm{pH}$ - low temperature quench allows for protein digestion and mass spectrometric analysis. 


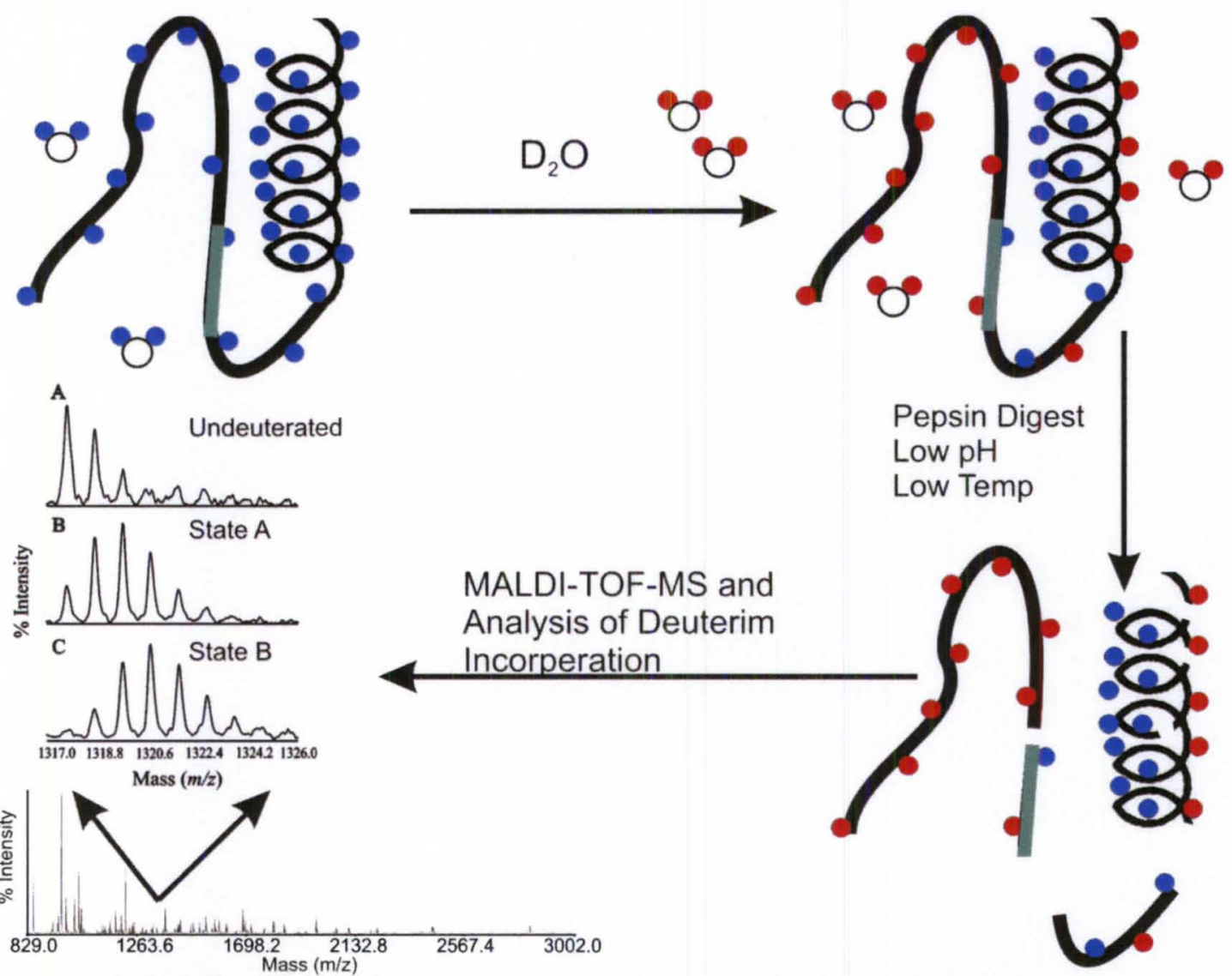

Figure 5: Schematic describing the processes involved in hydrogen-deuterium exchange coupled with MALDI-TOF-MS. The details are in-text.

The details of the method used for each HDX experiment are explained in the methods section of each respective chapter. Figure 5 is a pictorial representation to assist in visualizing the process. The starting material is a lyophilized protein containing hydrogens (blue circles) at all amide linkages. The protein is resuspended in $\mathrm{D}_{2} \mathrm{O}$, introducing the protein to a deuterium (red circles) environment. During the 10 minute room temperature incubation, the hydrogens are given time to exchange with the deuterons as described by the kinetic regime presented above. To quench the exchange, samples were placed on ice and trifluoroacetic acid was added dropping the $\mathrm{pH}$ to 2.2. The protease utilized was pepsin due to its ability to function at low $\mathrm{pH}$. After $10 \mathrm{~min}$, aliquots of the peptic digestion were quick-frozen in liquid nitrogen and stored cryogenically until analysis. 
Matrix was added to the sample spotted on MALDI plates for analysis. The spectra in Figure 5 are a representative FXIII- $\mathrm{A}_{2}$ pepsin digest. The three spectrum labeled A, B and C represent an undeuterated spectra (A), and two different states (B) and (C) where (C) displays the highest level of deuterium incorporation or exposure.

Hydrogen-deuterium exchange coupled with mass spectrometry provides an extremely powerful tool to analyze the conformational dynamics of proteins in solution. In the research presented HDX has successfully identified regions of FXIII that experience protection or exposure due to physiological $\mathrm{Ca}^{2+}$ concentrations, as well as after non-proteolytic activation. To approach the question of whether FXIII- $A_{2}$ adopts an open conformation when activated, FXIII$A_{2} \mathrm{HDX}$ was compared to transglutaminase 2 (TG2), an enzyme with the same domain structure as FXIII, in both an open and closed conformation. Lastly, the interface between FXIII-A $A_{2}$ and FXIII-B $B_{2}$ is not well defined and HDX has uncovered the regions of FXIII-A that become protected when in the heterotetramer conformation. 


\section{Research Goals}

FXIII- $A_{2}$ solution conformational dynamics is the basis of all research presented in this dissertation. The overarching goal is to gain a better understanding of the solution structure of FXIII-A and understand how it interacts with substrate. HDX was the primary method for studying FXIII-A conformational dynamics, but NMR was utilized in analyzing $\alpha \mathrm{C}(233-425)$ structure in solution with FXIII-A $A_{2}$ (described in chapter V).

In Chapter II, the FXIII-A $\mathrm{A}_{2}$ conformational dynamics journey starts by investigating the solution structure of FXIII-A in the presence of physiological $\mathrm{Ca}^{2+}(1 \mathrm{mM})(44)$. To determine whether the conformational changes seen in the presence of $\mathrm{Ca}^{2+}$ were $\mathrm{Ca}^{2+}$ specific, $\mathrm{Mg}^{2+}, \mathrm{Ba}^{2+}$ and $\mathrm{Cu}^{2+}$ were also investigated. Non-proteolytically $\mathrm{Ca}^{2+}$ activated $\mathrm{FXIIIa}{ }^{\mathrm{Ca}}$ was also analyzed by $\mathrm{HDX}$ and compared to FXIIIa $^{\mathrm{Na}}$. These HDX studies led to many new discoveries concerning the $\mathrm{Ca}^{2+}$ specific conformational dynamics of FXIII-A $\mathrm{A}_{2}$ both before ( 1 $\mathrm{mM} \mathrm{Ca}{ }^{2+}$ ) and after activation.

After Pinkas et al. reported that the transglutaminase TG2 could be trapped in an open conformation (49), HDX was utilized to determine if FXIII assumed a similar open conformation. Chapter III describes the conformational dynamics of TG2 in the activated state and also in the open state after inhibition with TG-DON. HDX has already been used to analyze FXIII-A $A_{2}$ in the activated 
state (Chapter II) and inhibited with the substrate peptide K9-Don (43). TG2 conformational dynamics have yet to be investigated by HDX and these analyses allow for a great comparison for FXIII-A to a known 'open' conformation.

The previous studies focused on the conformational dynamics of FXIII during the last phases of activation. Chapter IV took a step backward in the activation process to investigate the interaction between FXIII-A and FXIIIB-2. The FXIII- $A_{2} B_{2}$ heterotetramer plays a major role physiologically but the interface between the FXIII-A and FXIII-B subunits is not very well understood. HDX once again proved to be an invaluable tool in determining the regions of FXIII-A that become protected when bound to FXIII- $\mathrm{B}_{2}$.

When studying protein conformational dynamics, it is beneficial to establish an expression system for your protein of interest. The development of an expression system opens the doors to introducing mutations and/or the incorporation of isotopic labels for NMR analysis. The $\mathrm{aC}$ domain of fibrin is known to interact with FXIII- $A_{2} B_{2}$ and the $\alpha C(242-424)$ region aids in the dissociation of $F X I I I-A_{2} B_{2}(28)$. Chapter $V$ describes the expression system established and utilized to express ${ }^{15} \mathrm{~N}-\alpha \mathrm{C}(233-425)$. The ${ }^{15} \mathrm{~N}$-labeled $\alpha \mathrm{C}$ was then analyzed via ${ }^{15} \mathrm{~N}-\mathrm{HSQC}$ to determine if it adopted any compact structure when interacting with FXIII-A2. 
CHAPTER II

ROLE OF CALCIUM IN THE CONFORMATIONAL DYNAMICS OF FACTOR XIII ACTIVATION EXAMINED BY HYDROGEN/DEUTERIUM EXCHANGE COUPLED WITH MALDI-TOF MS

\section{Introduction}

Due to the critical role of FXIII in coagulation, there is a need to better understand the conditions that influence FXIII activation/activity as well as the structural dynamics of this enzyme system. Recombinant FXIII $A_{2}$ has been successfully crystallized (27), and the resultant structure exhibits well-defined and sequentially folded domains that include the activation peptide (1-37), the $\beta$ sandwich (38-184), the catalytic core (185-515), $\beta$-barrel 1 (516-628), and $\beta$ barrel 2 (629-730) Figure 6.

When FXIII is activated physiologically, thrombin (Ila) cleaves the $\mathrm{N}$ terminal activation peptide (residues 1-37). The presence of low $\mathrm{mM} \mathrm{Ca}^{2+}$ assists in the dissociation of the B subunits from plasma FXIII. In addition, $\mathrm{Ca}^{2+}$ promotes exposure of the catalytic C314 from both plasma and cellular FXIII resulting in an active $A_{2}$ dimer $\left(F X I I l a^{*}\right)(26,30,32)$. Like other transglutaminases (TGases), the active site of FXIII consists of a thiol-containing 
catalytic triad (C314, H373, and D396), but FXIII is unique in the fact that it exists as a dimer.

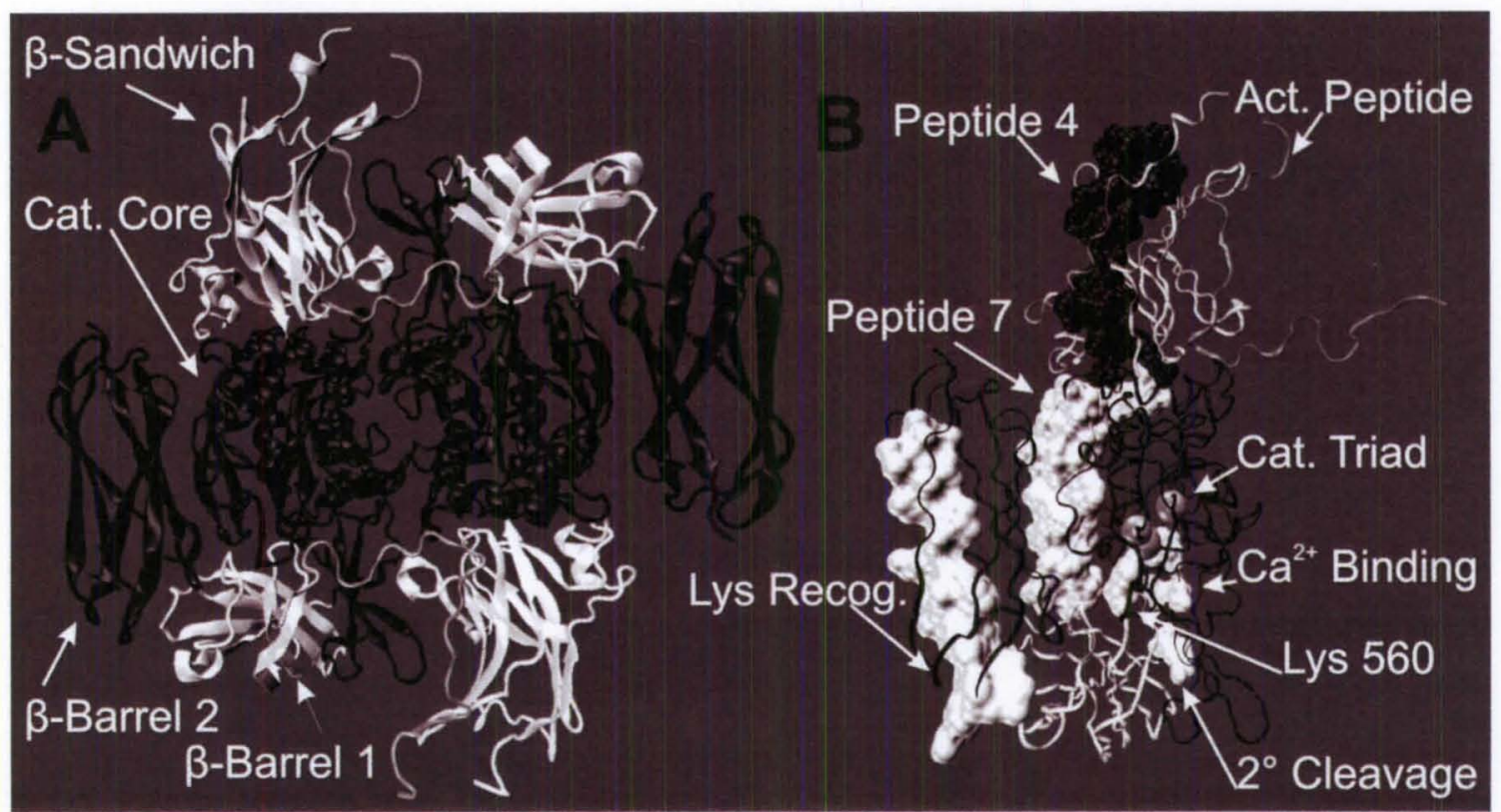

Figure 6: The FXIII $A_{2}$ zymogen $2.1 \AA$ crystal structure 1F13 (50). (A) A cartoon model to illustrate the intimate contact at the dimer interface with the domains being color coded (grey) activation peptide, (white) $\beta$-Sandwich, (black) catalytic core, (white) $\beta$-barrel 1 and (black) $\beta$-barrel 2. (B) Ribbon view of one FXIII-A monomer with the domains represented by the same colors as (A). The regions of interest are labeled: activation peptide (1-37), GIn recognition peptide 4 (72-97), GIn recognition peptide 7 (190-230), catalytic triad $(314,373$ and 396$)$, Tyrosine 560 , secondary cleavage site (513-514) and the proposed lysine recognition region (646-658). These figures were created using VMD (51).

In addition to lla proteolytic activation, FXIII $A_{2}$ can be non-proteolytically activated when the $\mathrm{Ca}^{2+}$ concentration is greater than $50 \mathrm{mM}\left(\mathrm{FXIIla}{ }^{\circ}\right.$ or $\mathrm{FXIIIa}{ }^{\mathrm{Ca}}$ ) (38-39). FXIII $A_{2}$ has also been observed in an activated state in the presence of $>150 \mathrm{mM} \mathrm{NaCl}$ and low $\mathrm{mM} \mathrm{Ca}^{2+}\left(\mathrm{FXIIla}^{\mathrm{Na}}\right)(40)$. A related form of nonproteolytic FXIII activation has been documented in platelets $(40,52-53)$. Thrombinstimulated platelets may exhibit increased $\mathrm{Ca}^{2+}$ levels that promote formation of an active intracellular FXIII species which has not been hydrolyzed at the R37G38 peptide bond. In plasma, these different forms of nonproteolytic activation are minimized by the presence of the $B_{2}$ subunits (54). Regardless of the mode of activation, FXIIla function can be hindered following hydrolysis at the 
secondary cleavage site (K513-S514) by thrombin (55-56). Occupation of a nearby site with $\mathrm{Ca}^{2+}$ and other select metals can help protect this secondary hydrolysis from occurring $(27,57)$.

Even though the catalytic C314 is only alkylated by iodoacetamide (IAA) after activation, $(33,58-59)$ the crystal structures for FXIIIalla and FXIIla ${ }^{\mathrm{Ca}}$ lack any major structural changes when compared to that of zymogen $(27,41-42)$. The Maurer lab has used MALDI-TOF mass spectrometry approaches coupled with hydrogen-deuterium exchange or chemical modification to probe FXIII conformation changes occurring in solution. During activation, portions of the FXIII catalytic core and the $A_{2}$ dimer interface were found to be more accessible to solvent $(45,60)$. By contrast, the addition of an inhibitory peptide with a glutamine isostere caused selected FXIII regions to become more protected from solvent (43). As expected from the secondary cleavage studies, $\mathrm{Ca}^{2+}$ binding hindered solvent exposure in a FXIII segment around 513-514. Inhibition at the active site could lead to further long-range decreases in HDX in this region.

A recent paper by Kristiansen and Andersen questions our use of borate buffer supplemented with $\mathrm{CaCl}_{2}$ for mass spectrometry based projects. Our HDX studies have not, however, been carried out under deactivated concentrations $\left(0.3 \mathrm{mM} \mathrm{CaCl}_{2}\right)$ as they presume, but at $1 \mathrm{mM} \mathrm{CaCl} 2$ following either proteolytic or nonproteolytic activation. According to their paper, FXIII $A_{2}$ that is activated nonproteolytically at $50 \mathrm{mM} \mathrm{CaCl}_{2}$ and then buffer exchanged into $1 \mathrm{mM} \mathrm{CaCl}_{2}$ maintains $90 \%$ of its enzyme activity (61). Moreover, many of the conformational events reported as being important for FXIII activation have been 
confirmed by HDX studies by Andersen and Faber using a buffer system of 200 $\mathrm{mM}$ Hepes, $150 \mathrm{mM} \mathrm{NaCl}$, and $50 \mathrm{mM} \mathrm{CaCl}_{2}$ (46).

The focus of our present HDX work was to further investigate the conformational changes that occur to FXIII due to monovalent and divalent cation binding. FXIII $\mathrm{A}_{2}$ was monitored in the presence of $\mathrm{Ca}^{2+}, \mathrm{Mg}^{2+}, \mathrm{Ba}^{2+}, \mathrm{Cu}^{2+}, \mathrm{Na}^{+}$, tetramethylammonium chloride $\left(\mathrm{TMAC}^{+}\right)$or ethylenediamine $\left(\mathrm{EDA}^{2+}\right)$ with concentrations ranging from physiological $\mathrm{Ca}^{2+}$ concentration, 1-2 $\mathrm{mM}$, up to 50$500 \mathrm{mM}$. On its own, the low $\mathrm{mM} \mathrm{Ca}^{2+}$ condition is unable to support FXIII activity. The HDX effects observed in this environment thus provide valuable information on early conformational changes needed in preparation for activation. Often these effects further increased as the $\mathrm{Ca}^{2+}$ concentration was raised to the levels required for nonproteolytic activation (50-500 mM). Several of the conformational changes observed with physiological $1 \mathrm{mM} \mathrm{Ca}^{2+}$ also occurred with the other divalent metals whereas other effects were distinct for a particular metal. In the current investigation, a novel method of nonproteolytic FXIII activation was characterized utilizing high concentrations of organic cations $\mathrm{TMAC}^{+}$or $\mathrm{EDA}^{2+}$. Knowledge gained from these different HDX studies will help identify the elusive structural roles of different divalent and monovalent cations. A greater understanding of the conformational dynamics of FXIII will aid in the development of new therapeutic strategies to control excessive bleeding, thrombosis, and/or atherosclerosis. 


\section{Materials and Methods}

Factor XIII Preparation and Synthetic Peptides

Recombinant human cellular factor XIII $A_{2}\left(F X I I I A_{2}\right)$ was generously provided by Dr. Paul Bishop (ZymoGenetics, Inc., Seattle, WA). After reconstituting the lyophilized FXIII in $18 \mathrm{M} \Omega$ deionized water, FXIII was buffer exchanged into $6.67 \mathrm{mM}$ borate at $\mathrm{pH}$ 8.3. The concentration of FXIII was determined on a Cary $100 \mathrm{UV} / \mathrm{vis}$ spectrophotometer. The absorbance was monitored at $280 \mathrm{~nm}$ and concentration calculated with the FXIII extinction coefficient of $1.49 \mathrm{ml} / \mathrm{mg} \mathrm{cm}$. Aliquots $(36 \mu \mathrm{l})$ of $16.7 \mu \mathrm{M}$ FXIII in $6.67 \mathrm{mM}$ borate were dried in a SpeedVac (Savant) and stored at $-70^{\circ} \mathrm{C}$ until future use.

The $\beta$ casein derived FXIII substrate peptide K9 (Ac-LGPGQSKVIG-OMe) was synthesized by Peptides International (Louisville, KY). K9 was reconstituted in $18 \mathrm{M} \Omega$ deionized water and the concentration was confirmed by quantitative amino acid analysis (AAA Service Laboratory, Inc., Boring, OR). Purity was assessed by HPLC and mass spectrometry approaches.

Transglutaminase Activity Assay

Factor XIII activity was determined using a modified version of the DadeBehring Berichrom Assay (62-63). Briefly, this assay utilizes a coupled reaction involving both FXIII and glutamate dehydrogenase (GDH). FXIIla reacts with the acyl-donor $\mathrm{K} 9$ releasing $\mathrm{NH}_{3}$ and the transglutaminase reaction concludes when the primary amine acyl-acceptor, glycine ethyl ester, forms an isopeptide bond with the $\mathrm{K} 9$ peptide. This transglutaminase activity is monitored via the 
conversion of $\alpha$-ketoglutarate and $\mathrm{NH}_{3}$ into glutamate in the presence of reducing equivalents of $\mathrm{NADH}$. The oxidation of $\mathrm{NADH}$ results in decreased absorbance at $340 \mathrm{~nm}$.

Dry FXIII aliquots were activated nonproteolytically in a total volume of 12 $\mu \mathrm{dH}_{2} \mathrm{O}$. The final working concentration was $50 \mu \mathrm{M} \mathrm{FXIII} \mathrm{in} 20 \mathrm{mM}$ Borate at $\mathrm{pH} 8.3$ with $50 \mathrm{mM} \mathrm{CaCl}_{2}, 50 \mathrm{mM} \mathrm{MgCl}_{2}$ with $2 \mathrm{mM} \mathrm{CaCl}_{2}, 50 \mathrm{mM} \mathrm{BaCl}_{2}$ with 2 $\mathrm{mM} \mathrm{CaCl}_{2}, 50 \mathrm{mM} \mathrm{CuCl}_{2}$ with $2 \mathrm{mM} \mathrm{CaCl}, 500 \mathrm{mM}$ ethylenediamine $\left(\mathrm{EDA}^{2+}\right.$ ) with $2 \mathrm{mM} \mathrm{CaCl}_{2}, 500 \mathrm{mM} \mathrm{NaCl}$ with $2 \mathrm{mM} \mathrm{CaCl}_{2}$, and $500 \mathrm{mM}$ tetramethylammonium chloride $\left(\mathrm{TMAC}^{+}\right)$with $2 \mathrm{mM} \mathrm{CaCl}$ ). FXIII was activated for $10 \mathrm{~min}$ at $37^{\circ} \mathrm{C}$ before adding $3 \mu \mathrm{l}$ to the assay. For each assay, the volume of activator reagent $(163 \mu \mathrm{l})$ and detector reagent $(250 \mu \mathrm{l})$ as well as the concentration of FXIII $(300 \mathrm{nM})$ and $\mathrm{K} 9(500 \mu \mathrm{M})$ were held constant (64). The assay contents were placed in a Cary $100 \mathrm{UV} / \mathrm{vis}$ spectrophotometer for $2 \mathrm{~min}$ at $37^{\circ} \mathrm{C}$ for equilibration before the $\mathrm{K} 9$ substrate peptide was added. After introducing the K9 peptide, the oxidation of $\mathrm{NADH}$ was monitored for 25 minutes at $340 \mathrm{~nm}$. Due to the coupled nature of this FXIII activity assay, there is a brief delay before consumption of NADH can be detected. The FXIII transglutaminase activity was later determined by the steepest part of the slope which correlates with enzyme catalyzed velocity ( $\Delta \mathrm{abs} / \mathrm{min}$ ). The final divalent and monovalent cation concentrations in the assays ( 0.3-3 mM) did not interfere with $\mathrm{GDH}$ reactivity. Using the molar absorptivity value for $\operatorname{NADH}\left(6220 \mathrm{M}^{-1} \mathrm{~cm}^{-1}\right.$ at $\left.340 \mathrm{~nm}\right)$, the transglutaminase based velocities were converted into $(\mu \mathrm{M} / \mathrm{min})$ and the final velocity values compared. For the studies involving the novel cations EDA ${ }^{2+}$, 
$\mathrm{Na}^{+}$, and $\mathrm{TMAC}^{+}$, transglutaminase activity was compared relative to that of FXIII ${ }^{\mathrm{Ca}}$ with \% FXIII ${ }^{\mathrm{Ca}}$ Activity defined as (FXIIla activity / FXIII ${ }^{\mathrm{Ca}}$ ) $\times 100$.

Standard Deviations of the mean for three independent trials were calculated. HDX Experimentation

There were two different groups of HDX samples, one monitoring the effects of low mM concentrations of divalent metals and the other examining nonproteolytic activation. Both groups utilized the dry buffer exchanged FXIII aliquots. To probe the effects of low mM divalent metals, $0.6 \mu$ of $20 \mathrm{mM}$ metalchloride $\left(\mathrm{CaCl}_{2}, \mathrm{MgCl}_{2}, \mathrm{BaCl}_{2}\right.$ or $\left.\mathrm{CuCl}_{2}\right)$ was added to the dry $\mathrm{FXIII}$ aliquot and evaporated to dryness in the SpeedVac and stored at $-70^{\circ} \mathrm{C}$. The following HDX protocol was then adapted from methodology established in the Komives laboratory (65-66). The dry FXIII aliquot was allowed to reach room temp before $12 \mu \mathrm{l}$ of $99.996 \% \mathrm{D}_{2} \mathrm{O}$ (Cambridge Isotope Laboratories) was added yielding a final working concentration of $50 \mu \mathrm{M}$ FXIII and $1 \mathrm{mM} \mathrm{Ca}^{2+}, \mathrm{Mg}^{2+}, \mathrm{Ba}^{2+}$ or $\mathrm{Cu}^{2+}$ in $20 \mathrm{mM}$ Borate at $\mathrm{pH}$ 8.3. The samples were incubated at room temperature for 10 minutes before the HDX was quenched by adding $120 \mu \mathrm{l}$ of chilled $0.1 \%$ TFA at $\mathrm{pH}$ 2.5. The quenched reaction was then immediately transferred to a tube containing activated pepsin bound to $6 \%$ agarose (Thermo Scientific, Rockford, IL). Pepsin digestion occurred for 10 min on ice. Following digestion, the reaction mix was centrifuged for $30 \mathrm{sec}$ to separate the FXIII digest from the pepsin beads. Three $8.2 \mu \mathrm{l}$ aliquots were immediately frozen in liquid $\mathrm{N}_{2}$ and each reaction condition was performed three times. 
When investigating nonproteolytically activated FXIII, aliquots were prepared as described previously. Divalent and monovalent cations $(<3 \mu \mathrm{l})$ were added to the dry FXIII aliquot for each of the activation conditions $\left(50 \mathrm{mM} \mathrm{Ca}^{2+}\right.$, $500 \mathrm{mM}$ ethylenediamine (EDA) with $2 \mathrm{mM} \mathrm{Ca}^{2+}, 500 \mathrm{mM} \mathrm{Na}^{+}$with $2 \mathrm{mM} \mathrm{Ca}^{2+}$, and $500 \mathrm{mM}$ tetramethylammonium chloride $\left(\mathrm{TMAC}^{+}\right)$with $\left.2 \mathrm{mM} \mathrm{Ca}^{2+}\right)$ and evaporated to dryness in a SpeedVac and stored at $-70^{\circ} \mathrm{C}$. After allowing the sample to reach room temperature, $12 \mu \mathrm{l} 99.996 \% \mathrm{D}_{2} \mathrm{O}$ was added and the $\mathrm{HDX}$ occurred at $37^{\circ} \mathrm{C}$ for $10 \mathrm{~min}$. The activated FXIII was digested and quenched as stated above.

HDX Analysis

A FXIII(a) HDX aliquot was thawed at room temperature and immediately mixed with an equal volume of $10 \mathrm{mg} / \mathrm{ml} \mathrm{a-cyano-hydroxycinnamic} \mathrm{acid} \mathrm{matrix}$ (a-CHCA) (Aldrich) in 1:1:1 ethanol/ $\mathrm{CH}_{3} \mathrm{CN} / 0.1 \% \mathrm{TFA}$ at $\mathrm{pH} 2.2$, and $0.5 \mu \mathrm{l}$ was spotted on a chilled MALDI plate. The sample spot was then quickly dried by placing the MALDI plate into a SpeedVac. The plate was then immediately inserted into the MALDI-TOF-MS (Voyager DE-Pro, Applied Biosystems). By limiting time for this procedure to $<5 \mathrm{~min}, \mathrm{HDX}$ back exchange is kept at a minimum. Spectra were collected in reflector mode with 256 shots/spectrum. All peptides in the peptic digest were previously identified by Brian T. Turner, Jr. (45) and/or confirmed by MS/MS analysis on an Applied Biosystems 4700. The MS/MS peptide identifications were performed using Applied Biosystems GPS software which utilized the Mascot database. Peptide identification was confirmed through observing the same peptide sequence in multiple independent 
digests, with an ion score preferably above 20 (peptic peptides Appendix C). After digestion by pepsin, the FXIII $A_{2}$ peptides identified represent $40 \%$

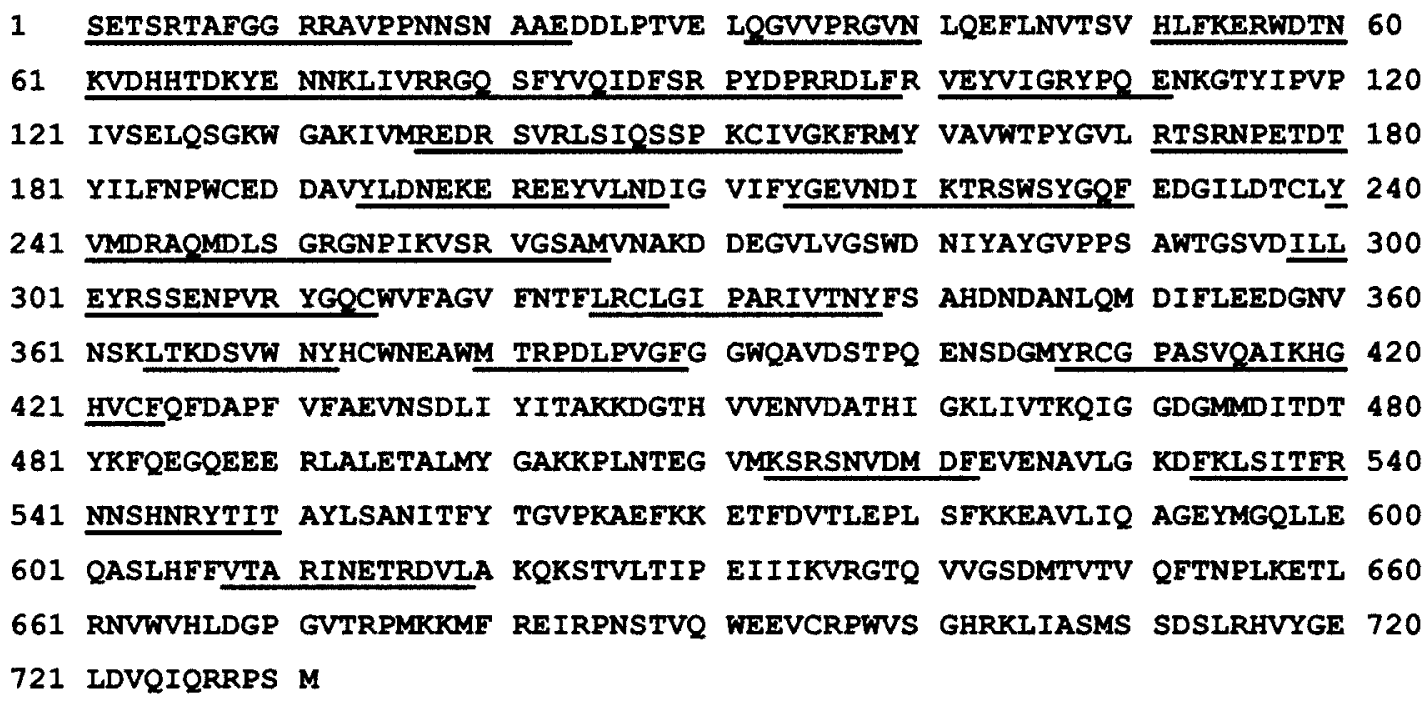

Figure 7: FXIII peptides observed by MALDI-MS after 10 minute pepsin digestion are underlined. The underlined peptides represent $40 \%$ coverage.

coverage (Figure 7). These peptides focus on key FXIII regions found within the $\beta$-sandwich, the catalytic core, and the $\beta$-barrel 1 region. Additional studies with acid dependent type XIII protease did not significantly improve sequence coverage. All the MALDI spectra derived from pepsin digests were analyzed using Data Explorer (Applied Biosystems) and calibrated using two singly protonated reference peptides; monoisotopic mass $850.4787 \mathrm{Da}$ (residues 535541) and quadraisotopic mass of 1375.7097 Da (residues 220-230).

Deuterium incorporation for each isotopic cluster was quantified as described by Sabo et al. (67). To determine the change in deuteration for FXIII under different conditions the percent deuteration for each peptide was calculated using equation 1 : 
*difference $=\frac{\left(D-D_{F X 1 L}\right)}{D_{\max }} \times 100$

where $D_{F X I I I}$ is the amount of deuterium incorporated in FXIII zymogen, $D$ is the amount of deuterium incorporation in FXIII under different conditions and $D_{\max }$ is the theoretical maximum amount of deuterium incorporation for the given peptide under these conditions. The theoretical maximum depends on the final percentage of $\mathrm{D}_{2} \mathrm{O}$ under quench conditions (4.5\%) and accounts for all exchangeable backbone amide protons as well as a fraction of $\mathrm{N}$-terminal, $\mathrm{C}$ terminal and side chain exchangeable protons. In accordance with previous HDX data analysis, percent differences greater than $4.5 \%$ are considered significant $(45,67-69), 3-4.5 \%$ is moderate and $<3 \%$ is modest. 


\section{Results}

1 mM Divalent Metals and FXIII - HDX-MS

Human plasma contains 1-2 mM calcium, therefore the solvent accessibility of FXIII zymogen (without metals) was compared to that of FXIII in the presence of $1 \mathrm{mM} \mathrm{Ca}^{2+}, \mathrm{Mg}^{2+}, \mathrm{Ba}^{2+}$ or $\mathrm{Cu}^{2+}$. All studies would thus be carried out in the presence of a physiological cation concentration environment. Prior knowledge about these divalent cations includes the following derived from studies carried out at concentrations from 0.1 to $10 \mathrm{mM}^{\mathrm{M}} \mathrm{Mg}^{2+}$ is smaller in size than $\mathrm{Ca}^{2+}$ and does not promote the conformation-based fluorescence changes that are observed upon $\mathrm{Ca}^{2+}$ binding to FXIII (70). $\mathrm{Mg}^{2+}$ also does not support

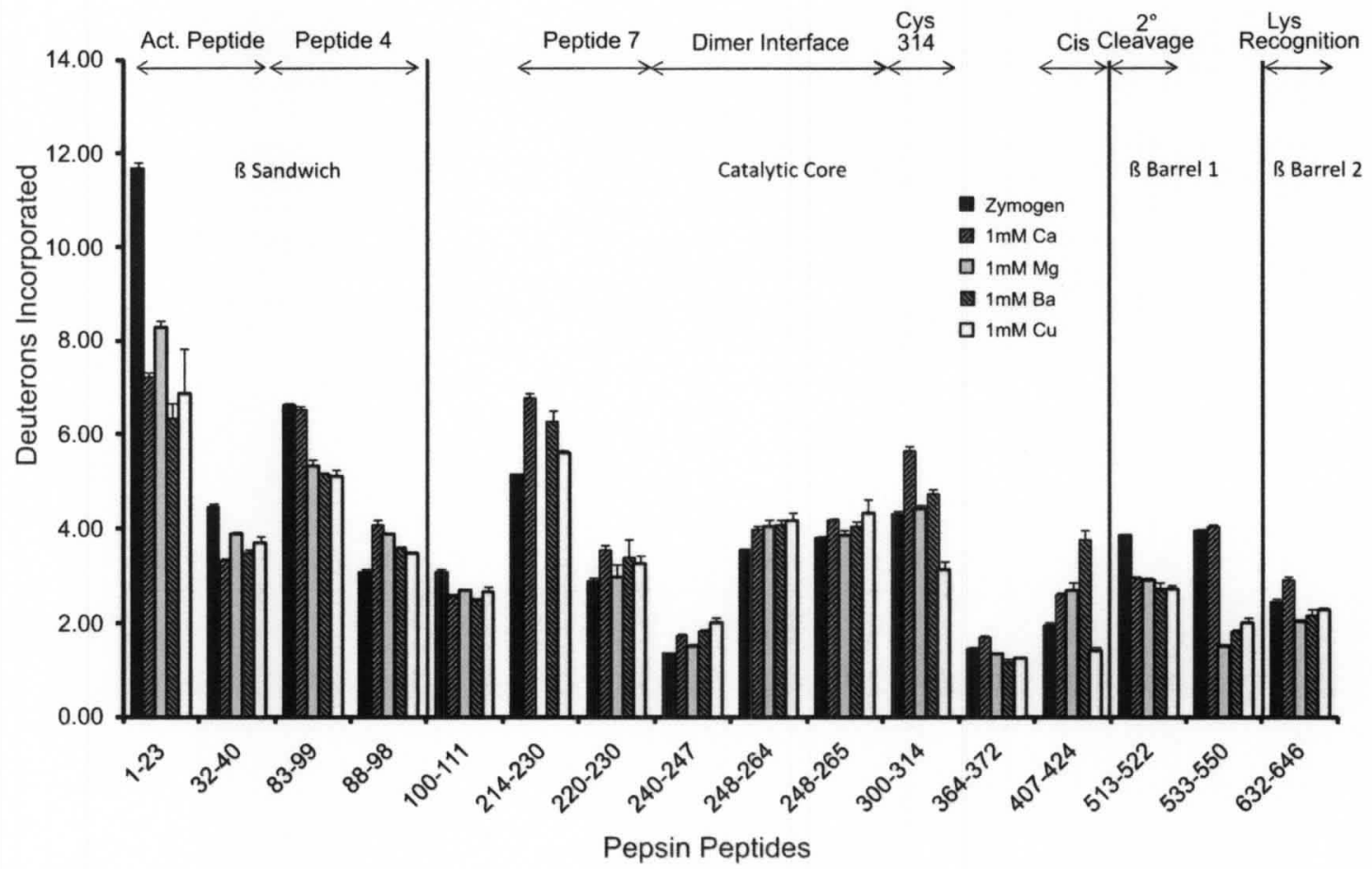

Figure 8: The number of deuterons incorporated at $10 \mathrm{~min}$ for FXIII under the following conditions zymogenic (no metal) FXIII (black), $1 \mathrm{mM} \mathrm{Ca}^{2+}$ (up diagonal), $1 \mathrm{mM} \mathrm{Mg}^{2+}$ (grey), $1 \mathrm{mM} \mathrm{Ba}^{2+}$ (down diagonal) and $1 \mathrm{mM} \mathrm{Cu}^{2+}$ (white). The top of the graph illustrates regions of interest in FXIII. Error bars represent the standard deviation of the mean for 3 independent trials. 
FXIIIalla activity but does protect against thrombin-catalyzed proteolysis at the secondary cleavage site $(57,70)$. Larger in size than $\mathrm{Ca}^{2+}, \mathrm{Ba}^{2+}$ exhibits no protection against secondary cleavage and modest ability to support FXIIIalla activity $(33,57) . \mathrm{Cu}^{2+}$ is a transition metal that is closer in size to $\mathrm{Ca}^{2+}$ and exerts no protection of the K513-S514 site but can support some FXIIIalla activity (33, $57)$.

For the current conformational studies on FXIII $A_{2}$, Table 1 displays changes in deuterium incorporation of FXIII zymogen compared to FXIII-(Ca, Mg,

Table 1: Changes in Percent Deuteration: FXIII with $1 \mathrm{mM}$ Metal Relative to FXIII Zymogen ${ }^{a}$.

\begin{tabular}{|c|c|c|c|c|c|}
\hline Residues & $\begin{array}{l}\text { Theo } \\
D_{\max } b\end{array}$ & $1 \mathrm{mM} \mathrm{Ca}^{2+}$ & $1 \mathrm{mM} \mathrm{Mg} \mathrm{Mg}^{2+}$ & $1 \mathrm{mM} \mathrm{Ba}^{2+}$ & $1 \mathrm{mM} \mathrm{Cu}^{2+}$ \\
\hline $1-23$ & 23.3 & $-19.1^{c}$ & -14.6 & -22.9 & -20.7 \\
\hline $32-40$ & 8.9 & -12.7 & -6.6 & -10.9 & -8.5 \\
\hline $83-99$ & 23.2 & -0.4 & -5.6 & -6.3 & -6.5 \\
\hline $88-98$ & 10.9 & 9.2 & 7.3 & 4.6 & 3.5 \\
\hline $100-111$ & 11.8 & -4.1 & -3.4 & -5.0 & -3.6 \\
\hline $214-230$ & 18.8 & 8.8 & $\mathrm{n} / \mathrm{a}^{d}$ & 6.2 & 2.5 \\
\hline $220-230$ & 12.3 & 5.6 & 0.7 & 4.3 & 3.1 \\
\hline $240-247$ & 7.5 & 5.3 & 2.2 & 6.3 & 8.7 \\
\hline $248-264$ & 15.9 & 2.9 & 3.3 & 3.6 & 4.0 \\
\hline $248-265$ & 18.8 & 2.1 & 0.3 & 1.4 & 2.8 \\
\hline $300-314$ & 15.8 & 8.7 & 0.8 & 3.0 & -7.4 \\
\hline $364-372$ & 8.4 & 3.5 & -1.0 & -2.9 & -2.3 \\
\hline $407-424$ & 18.8 & 3.6 & 4.0 & 9.7 & -2.8 \\
\hline $513-522$ & 9.7 & -9.0 & -9.7 & -11.3 & -11.6 \\
\hline $533-550$ & 20.1 & 0.5 & -6.0 & -6.4 & -6.8 \\
\hline $632-646$ & 14.7 & 3.2 & -2.8 & -1.9 & -1.1 \\
\hline \multicolumn{6}{|c|}{ 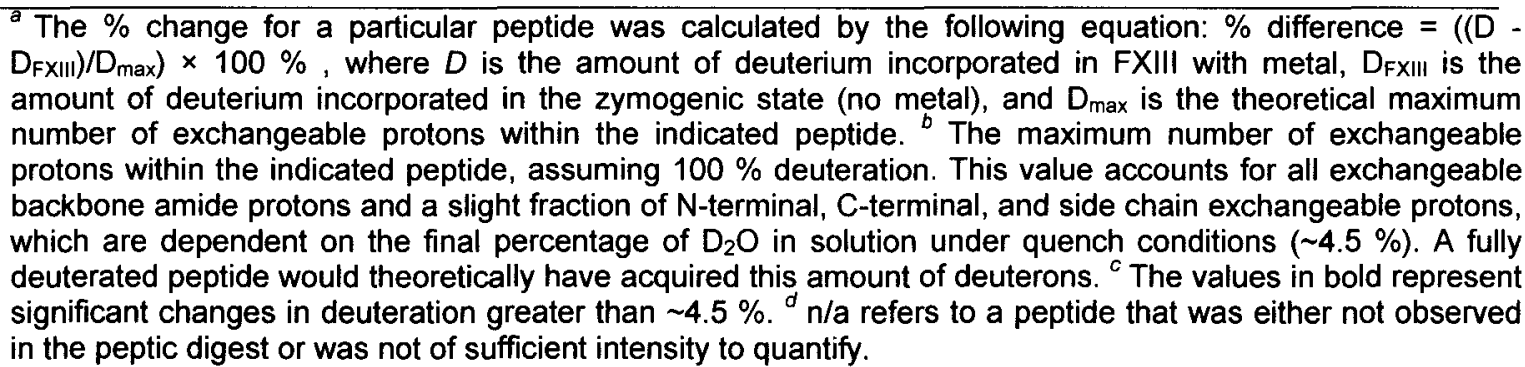 } \\
\hline
\end{tabular}


$\mathrm{Ba}$ and $\mathrm{Cu}$ ). The total deuterium incorporated for each pepsin-derived peptide is displayed in Figure 8. Note that the top of the chart highlights the different FXIII regions where the peptides reside.

Within the $\beta$ sandwich domain, the two main regions of interest are the activation peptide (1-37) and the putative Gln substrate recognition site (72-97), also known as peptide 4. After 10 minutes of HDX, the activation peptide became more protected from solvent relative to its values in the zymogen state of FXIII. Such protection was observed for all the metals tested and ranged from $6.6 \%$ for FXIII-Mg to $-22.9 \%$ for FXIII-Ba. The peptide 4 segment (71), is represented by the pepsin derived peptides $83-99$ and 88-98. For peptide 83-99, changes in deuteration ranged from $-0.4 \%$ for FXIII-Ca to $-6.5 \%$ for FXIII-Cu. By contrast, the region $88-98$ became more exposed in the presence of all metals ranging from $3.5 \%$ for $\mathrm{FXIII-Cu}$ to $9.2 \%$ for $\mathrm{FXIII-Ca}$. The different responses for $83-99$ versus $88-98$ suggest that the amide protons from residues $83-87$ and/or 99 may be responsible for the HDX protection observed for 83-99 in the presence of divalent metals (Figure 8). Further support for the importance of residue 99 comes from the observation that the next FXIII segment 100-111 exhibits solvent protection in the presence of all the cations tested.

The catalytic core contains several regions of interest. Peptide 7 (190230), another putative glutamine-substrate region (71), displayed increased deuterium incorporation for metals when compared to zymogen. FXIII-Ca was the most exposed for peptides $214-230$ and $220-230$ followed by FXIII-Ba, FXIII$\mathrm{Cu}$, and finally FXIII-Mg. The dimer interface between the two FXIII-A monomers 
(residues 240-265) was another region where increases in deuterium incorporation for all the metals was observed. Exposure within the dimer interface (240-247) for FXIII-(Ca, Mg, Ba and $\mathrm{Cu}$ ) when compared to zymogen was $5.3,2.2,6.3$, and $8.7 \%$ respectively. As with the peptide 7 region, FXIII-Mg exhibited the smallest effect. Interestingly, protection from solvent (-3.4 to $-5.0 \%)$ was observed for the residues $100-111$. The $\mathrm{C}$-terminal portion of this segment is located at the dimer interface; however, the more $\mathrm{N}$-terminal portion is found in a $\beta$-sandwich region.

The catalytic C314 is found within segment $300-314$ and the catalytic $\mathrm{H} 373$ is found one residue outside $364-372$. For the $364-372$ segment containing the $\mathrm{H} 373,1 \mathrm{mM} \mathrm{Ca}^{2+}$ showed a moderate $3.5 \%$ increase in HDX exposure whereas the other metals all showed a modest protection. By contrast, FXIII-Ca displayed considerable exposure (8.7\%) for 300-314 when compared to FXIII-(Mg and $\mathrm{Ba}$ ) with $0.8 \%$ and $3.0 \%$ exposure, respectively. FXIII-Cu was the only condition found to be protected in this region (-7.4\%). FXIII-Cu also became modestly protected in the region of $407-424$ which is N-terminal to the FXIII $\mathrm{Ca}^{2+}$ binding site $(-2.84 \%)$ and makes contacts across the dimer interface. FXIII-(Ca, Mg, and $\mathrm{Ba})$, however, displayed increases in deuterium incorporation for this segment. The $407-424$ segment may be influenced by a nearby nonprolyl cis peptide bond that is proposed to play a role in FXIII activation (50). The $\beta$-barrels contain the secondary cleavage site (513-514) and the lysine recognition site (646-658) (72-73). FXIII-(Ca, Mg, Ba and $\mathrm{Cu}$ ) all displayed protection within $513-522$ when compared to zymogen (-9.0 to $-11.6 \%)$. The 
nearby FXIII $533-550$ is not influenced by $1 \mathrm{mM}$ Ca whereas it is significantly solvent protected in FXIII-(Mg, Ba, and $\mathrm{Cu})$ at levels of -6.0 to $-6.8 \%$. Peptide 632-646 is on the $\mathrm{N}$-terminal end of the proposed lysine recognition site and is moderately exposed in FXIII-Ca (3.6\%) (73). All other conditions showed little if any protection in this region of $\beta$-barrel 2 .

TGase Activity of Nonproteolytically Activated FXIII

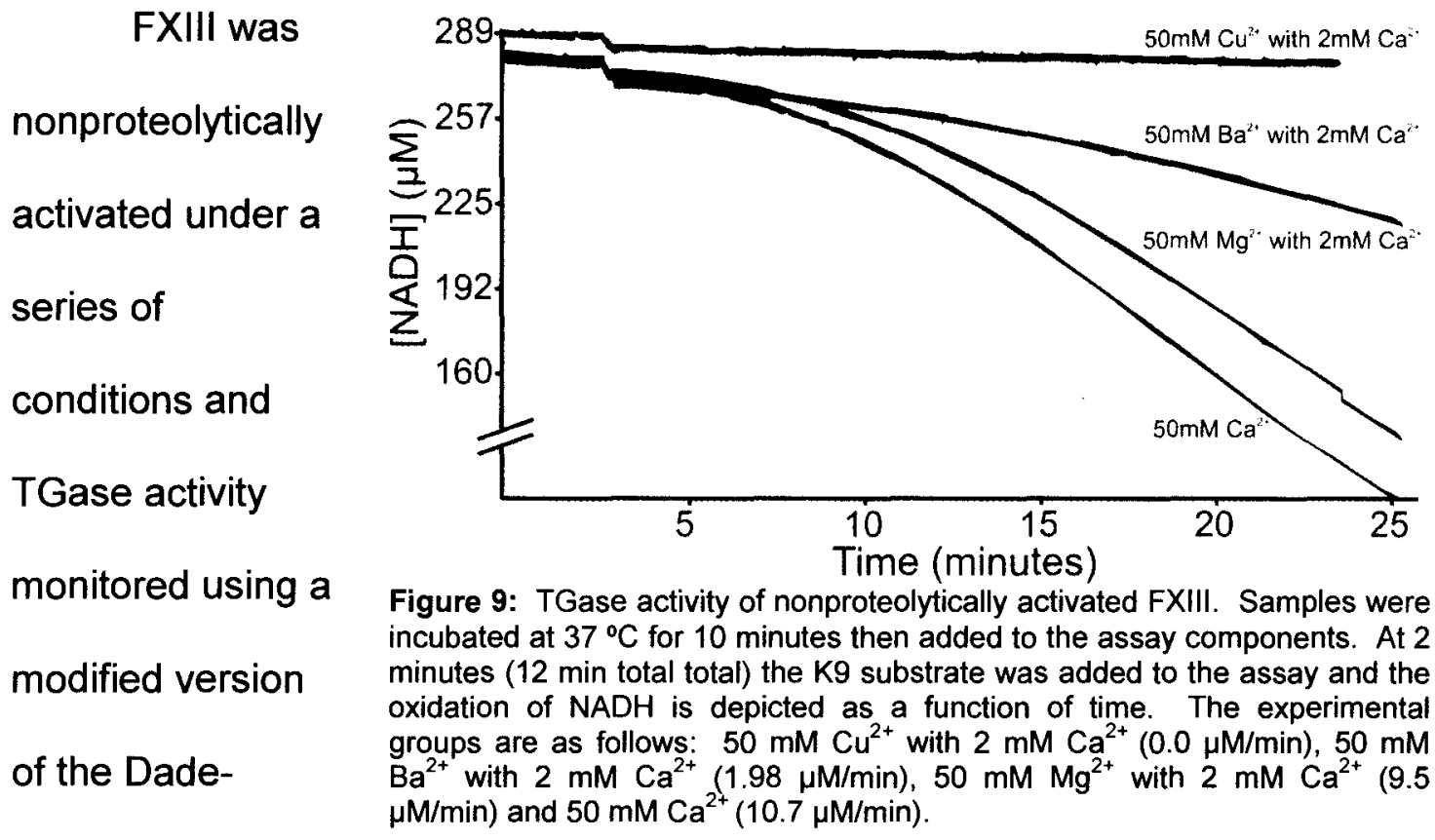

Behring

Berichrom assay (64). When incubated in the presence of several different divalent metals including $50 \mathrm{mM} \mathrm{Ca}^{2+}, \mathrm{Mg}^{2+}, \mathrm{Ba}^{2+}$ or $\mathrm{Cu}^{2+}$, only $\mathrm{Ca}^{2+}$ displayed activity (data not shown). After changing the activation conditions to include 2 $\mathrm{mM} \mathrm{Ca}{ }^{2+}$ with $50 \mathrm{mM} \mathrm{Mg}^{2+}, \mathrm{Ba}^{2+}$ or $\mathrm{Cu}^{2+}$, minor activity was observed in $\mathrm{Mg}^{2+}$ and $\mathrm{Ba}^{2+}$ but not $\mathrm{Cu}^{2+}$ (Figure 9). Factor XIII activity was then monitored using a series of other nonproteolytic techniques. FXIIIa ${ }^{\mathrm{Ca}}$ was compared to FXIII activated in the presence of $500 \mathrm{mM} \mathrm{EDA}^{2+}\left(\mathrm{FXIIla}^{\mathrm{EDA}}\right)$ with $2 \mathrm{mM} \mathrm{Ca}^{2+}, 500 \mathrm{mM}$ 
$\mathrm{Na}^{+}$with $2 \mathrm{mM} \mathrm{Ca}^{2+}\left(\mathrm{FXIIIa}^{\mathrm{Na}}\right)$ and $500 \mathrm{mM} \mathrm{TMAC}^{+}$with $2 \mathrm{mM} \mathrm{Ca}^{2+}\left(\mathrm{FXIIIa}^{\mathrm{TMAC}}\right)$. Using the slope ( $\mu \mathrm{M}$ NADH consumed / min) as an indicator of activity, EDA ${ }^{2+}$, $\mathrm{Na}^{+}$and $\mathrm{TMAC}^{+}$displayed an increase in FXIII activity of $291 \pm 27.7 \%, 156.7 \pm$ $16.3 \%$ and $194.8 \pm 5.5 \%$, respectively when compared to $\mathrm{FXIIIa}^{\mathrm{Ca}}$.

Nonproteolytically activated FXIIIa - HDX-MS

The conformational dynamics of nonproteolytically activated FXIII were also probed using HDX-MS. Due to peak suppression in the MALDI, it was not possible to quantify the deuterium incorporated into FXIIIa ${ }^{\text {EDA }}$ and FXIIIa ${ }^{\text {TMAC }}$. Table 2 thus displays changes in deuterium incorporation of FXIII zymogen compared to $\mathrm{FXIII}$ in $2 \mathrm{mM} \mathrm{Ca}^{2+}, \mathrm{FXIIIa}^{\mathrm{Ca}}$ and $\mathrm{FXIIla}{ }^{\mathrm{Na}}$. The total deuterium incorporated in each peptide is displayed in Figure 10.

Table 2: Changes in Percent Deuteration: Nonproteolytically Activated FXIII Relative to FXIII Zymogen ${ }^{a}$.

\begin{tabular}{|c|c|c|c|c|}
\hline Peptide & $\begin{array}{l}\text { Theo } \\
D_{\max }{ }^{b}\end{array}$ & $2 \mathrm{mM} \mathrm{Ca}{ }^{2+}$ & $50 \mathrm{mM} \mathrm{Ca}{ }^{2+}$ & $\begin{array}{l}50 \mathrm{mM} \mathrm{Na} \\
\text { with } 2 \mathrm{mM} \\
\mathrm{Ca}^{2+}\end{array}$ \\
\hline $1-23$ & 23.3 & $-16.3^{c}$ & -8.3 & $\mathrm{n} / \mathrm{a}$ \\
\hline $32-40$ & 8.9 & -12.1 & -12.7 & -12.7 \\
\hline $88-98$ & 10.9 & -0.9 & -1.9 & -1.3 \\
\hline $100-111$ & 11.8 & -4.5 & -6.2 & -5.9 \\
\hline $220-230$ & 12.3 & 9.3 & 13.2 & 17.1 \\
\hline $240-247$ & 7.5 & 7.2 & 18.2 & 19.8 \\
\hline $248-264$ & 15.9 & 1.4 & $\mathrm{n} / \mathrm{a}^{d}$ & 2.5 \\
\hline $248-265$ & 18.8 & 4.7 & 2.2 & 4.5 \\
\hline $300-314$ & 15.8 & 7.1 & 7.7 & 11.7 \\
\hline $407-424$ & 18.8 & 0.2 & -0.1 & -0.8 \\
\hline $513-522$ & 9.7 & 0.0 & 1.7 & 1.9 \\
\hline $533-550$ & 20.1 & 2.6 & 16.8 & $\mathrm{n} / \mathrm{a}$ \\
\hline \multicolumn{5}{|c|}{ 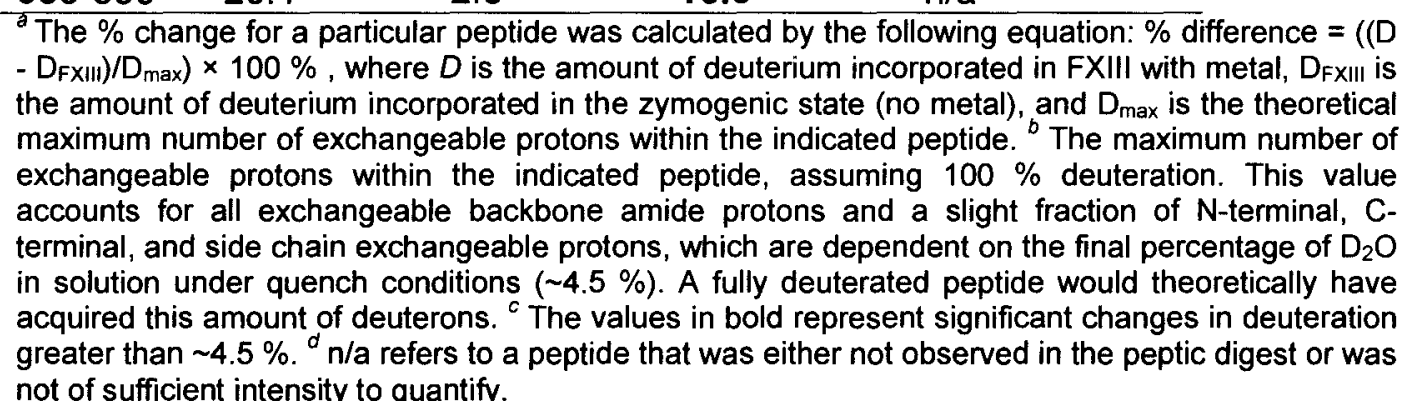 } \\
\hline
\end{tabular}


To ensure activity was achieved during HDX, the same activation conditions used to confirm nonproteolytic activity were utilized for HDX analysis, except $\mathrm{D}_{2} \mathrm{O}$ was substituted for $\mathrm{H}_{2} \mathrm{O}$. The extent of deuterium incorporation may vary relative to previous work since this $\mathrm{HDX}$ project was conducted at $37^{\circ} \mathrm{C}$. When activated in the presence of $50 \mathrm{mM} \mathrm{Ca}^{2+}$ or in $500 \mathrm{mM} \mathrm{Na}^{+}$with $2 \mathrm{mM}$ $\mathrm{Ca}^{2+}$, the $\beta$ sandwich activation peptide of $\mathrm{FXIIIa}{ }^{\mathrm{Ca}}$ and $\mathrm{FXIIIa}{ }^{\mathrm{Na}}$ was more protected than in FXIII zymogen. This effect was seen in the $-12.7 \%$ change found for residues $32-40$ in both FXIIIa ${ }^{\mathrm{Ca}}$ and FXIIIa ${ }^{\mathrm{Na}}$. Residues 100-111 fall within $4 \AA$ of the dimer interface and they too displayed protection of $-6.2 \%$ and $5.9 \%$ for $\mathrm{FXIIIa}^{\mathrm{Ca}}$ and $\mathrm{FXIIIa}{ }^{\mathrm{Na}}$, respectively when compared to zymogen.

Peptide 7 within the catalytic core became notably exposed when nonproteolytically activated leading to a $13.2 \%$ increase in deuterium incorporation for $\mathrm{FXIII}^{\mathrm{Ca}}$ and a $17.1 \%$ increase for $\mathrm{FXIIIa}{ }^{\mathrm{Na}}$ at residues $220-230$. The FXIII dimer interface (240-247) within the catalytic core experienced an 18.2 $\%\left(\right.$ FXIIIa $\left.{ }^{\mathrm{Ca}}\right)$ and $19.8 \%\left(\mathrm{FXIIIa}^{\mathrm{Na}}\right)$ increase in solvent accessibility when activated. Increased accessibility was also evident within the catalytic cysteine containing segment (300-314). This region changed $7.7 \%$ and $11.7 \%$ for $\mathrm{FXIIIa}^{\mathrm{Ca}}$ and FXIIIa ${ }^{\mathrm{Na}}$ respectively when compared to zymogen (Table 2). These various changes in solvent exposure were all greater than what could be observed under a more physiological 1-2 $\mathrm{mM} \mathrm{Ca}^{2+}$ concentration. Such results suggest that these two FXIII regions (peptide 7 and dimer interface) become more solvent accessible as the cation concentration is increased (Figure 5). In a $\mathrm{Na}^{+}$-containing environment, low $\mathrm{mM}$ calcium is required for activation. 
Events occurring within the $\beta$ barrels are also important to consider.

Unlike the studies done in the presence of $1 \mathrm{mM} \mathrm{Ca}^{2+}$, no substantial changes in solvent exposure were observed for $\beta$-barrel 1 residues 513-522 under the nonproteolytic activation conditions at $37^{\circ} \mathrm{C}$. By contrast, accessibility to solvent increased steadily from 2 to $50 \mathrm{mM} \mathrm{Ca}^{2+}$ for the neighboring segment $533-550$ (2.6 to $16.8 \%$ ). Within $\beta$-barrel 2, peptide $632-646$ is located $N$-terminal to the lysine recognition region (646-658) and exhibited an increased deuterium incorporation of $6.2 \%$ with FXIIIa ${ }^{\mathrm{Na}}$.

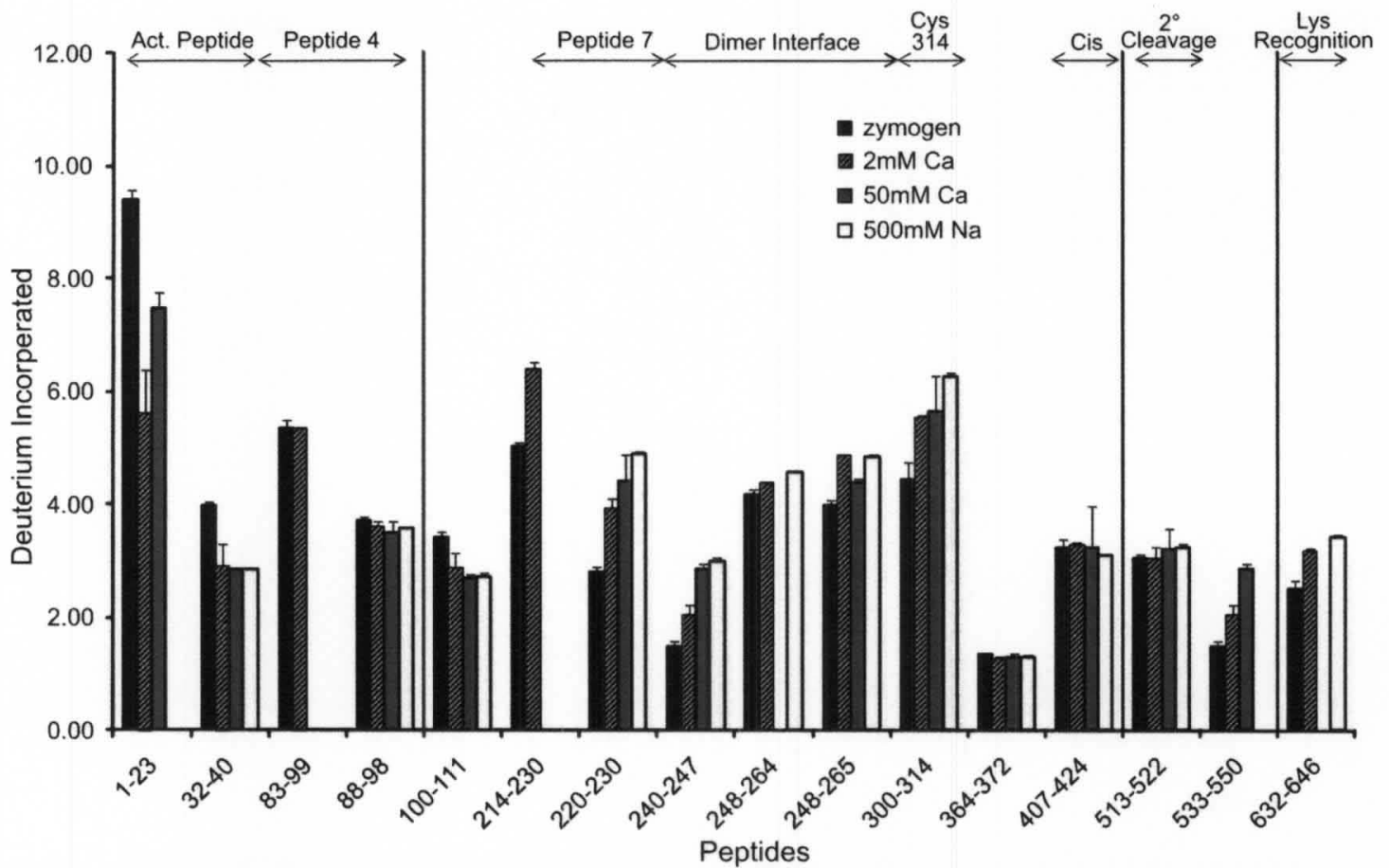

Figure 10: The number of deuterons incorporated after $10 \mathrm{~min}$ at $37^{\circ} \mathrm{C}$ for nonproteolytically activated FXIII. Deuterium exchange was monitored under the following conditions zymogenic (no metal) FXIII (black), $2 \mathrm{mM} \mathrm{Ca}^{2+}$ (up diagonal), FXIIla ${ }^{\mathrm{Ca}} 50 \mathrm{mM} \mathrm{Ca}^{2+}$ (grey), $\mathrm{FXIIIa}^{\mathrm{Na}} 500 \mathrm{mM} \mathrm{Na}^{+}$with $2 \mathrm{mM} \mathrm{Ca}^{2+}$ (whites). The top of the graph illustrates regions of interest in FXIII. Error bars represent the standard deviation of the mean for 3 independent trials. 


\section{Discussion}

Calcium plays a vital role in the activation and regulation of cellular $\left(A_{2}\right)$ and plasma $\left(A_{2} B_{2}\right)$ FXIII $(33,57-58,74)$. HDX provides an effective solutionbased approach for dissecting out how $\mathrm{Ca}^{2+}$ and other divalent and monovalent species $\left(\mathrm{Mg}^{2+}, \mathrm{Ba}^{2+}, \mathrm{Cu}^{2+}, \mathrm{Na}^{+}, \mathrm{EDA}^{2+}, \mathrm{TMAC}^{+}\right)$participate in the conformational dynamics of this transglutaminase.

Impact on $\mathrm{Ca}^{2+}$ binding site and surrounding regions

The FXIII ion binding helix (485-501) is located within $10 \AA$ of $\beta$-barrel 1 and also makes contact with the other FXIII-A monomer across the dimer interface $(42,55,75)$. See golden helix in Figure 11 . The primary calcium binding site involves FXIII residues Asn436, Asp438, Ala457, Glu485 and Glu490 (Figure 11 pink sticks) and site-directed mutagenesis studies have demonstrated that when the glutamate residues are removed, FXIII sensitivity to $\mathrm{Ca}^{2+}$ decreases $(27,55-56)$. According to Lewis et al., when $\mathrm{Ca}^{2+}$ concentrations are $<2.5 \mathrm{mM}$, there is one $\mathrm{Ca}^{2+}$ bound per FXIII-A, but there are up to 8 low affinity sites when the concentration is raised above that threshold (70). The presence of strong and weak $\mathrm{Ca}^{2+}$ binding sites has also been confirmed by ${ }^{43} \mathrm{Ca}$ NMR studies (76). Our studies to determine the conformational dynamics of FXIII $A_{2}$ in $1 \mathrm{mM}$ metal were performed to mimic physiological $\mathrm{Ca}^{2+}$ concentrations and to monitor events where a single $\mathrm{Ca}^{2+}$ should target the higher affinity metal site on FXIII. The other divalent metals were examined under these same $1 \mathrm{mM}$ concentration values. 
Although sequence coverage was not observed for the FXIII calcium binding site, $40 \%$ of the enzyme including a segment containing (513-514), a secondary cleavage site that is protected from hydrolysis in the presence of calcium was observed (57). This site of proteolytic degradation (red sticks) is located just 12 residues down from the ion binding helix (485-510). See Figure 11. All metals tested at $1 \mathrm{mM}$ concentration $\left(\mathrm{Ca}^{2+}, \mathrm{Mg}^{2+}, \mathrm{Ba}^{2+}\right.$, and $\left.\mathrm{Cu}^{2+}\right)$ demonstrated solvent protection around peptide 513-522. This finding was surprising considering that Mary et al. reported $\mathrm{Ba}^{2+}$ and $\mathrm{Cu}^{2+}$ provided no protection against $2^{\circ}$ cleavage (57).

Unlike the 513-522 region, $1 \mathrm{mM} \mathrm{Ca}^{2+}$ did not show any deuterium protection for the nearby 533-550 segment (Figure 11 purple) which is part of an anti-parallel $\beta$-sheet that runs adjacent to $513-522$. By contrast, the other divalent metals $\mathrm{Mg}^{2+}, \mathrm{Ba}^{2+}$ and $\mathrm{Cu}^{2+}$ all showed substantial protection within 533-550 when compared to FXIII in $1 \mathrm{mM} \mathrm{Ca}^{2+}$. The $\mathrm{HDX}$ results suggest that $\mathrm{Ba}^{2+}$ and $\mathrm{Cu}^{2+}$ exert a greater influence on 533-550 with additional effects on 513-522; however, these events are not sufficient to protect FXIII from proteolysis. $1 \mathrm{mM}$ $\mathrm{Ca}^{2+}$ may exert a more direct influence on the secondary cleavage site 513-514. The ability of $1 \mathrm{mM} \mathrm{Mg}^{2+}, \mathrm{Ba}^{2+}$ and $\mathrm{Cu}^{2+}$ to influence $\mathrm{FXIII}$ dynamics brings up the question of whether such divalent metals could support nonproteolyticallyderived TGase activity as already observed with $\mathrm{Ca}^{2+}$. Solutions containing 50 $\mathrm{mM} \mathrm{Mg}^{2+}$ or $\mathrm{Ba}^{2+}$, however, all required the added presence of $2 \mathrm{mM} \mathrm{Ca}^{2+}$ to produce FXIII activity following 10 minutes of nonproteolytic activation at $37^{\circ} \mathrm{C}$. Furthermore, their levels of enzymatic activity were all less than those for $50 \mathrm{mM}$ 
$\mathrm{Ca}^{2}$. By contrast, Factor XIII in $50 \mathrm{mM} \mathrm{Cu}^{2+}$, even in the presence of $2 \mathrm{mM} \mathrm{Ca}^{2+}$, was not able to yield TGase activity. From these studies, it is evident that a series of divalent metals can support FXIII activation and TGase activity but low $\mathrm{mM} \mathrm{Ca}{ }^{2+}$ is still needed to occupy what is likely the high affinity ion binding site on FXIII.

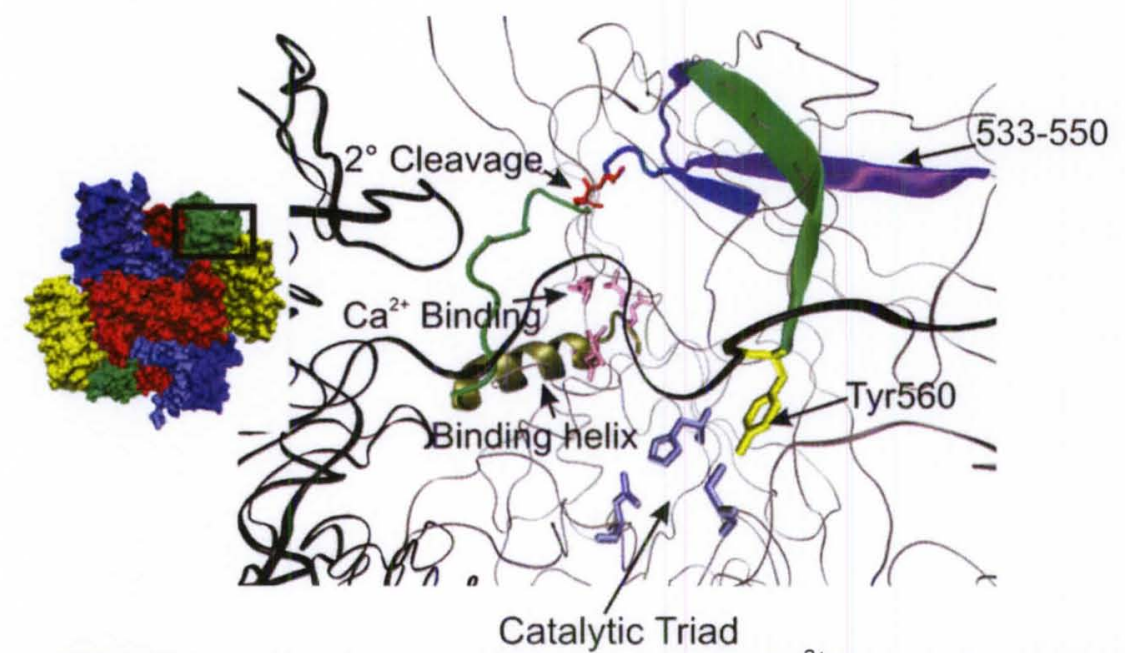

Figure 11: Illustration of the regions within the catalytic core and the $\mathrm{Ca}^{2+}$ binding site mentioned in the conformational dynamics of FXIII $A_{2}$ (1F13). FXIII $A_{2}$ (inset) has each domain labeled: $\beta$-sandwich (blue), catalytic core (red), $\beta$-barrel 1 (green) and $\beta$-barrel 2 (yellow) with the box representing the region of interest. The $\mathrm{Ca}^{2+}$ binding site (pink) and the ion binding helix (gold) are connected to the secondary cleavage site 513-514 (red sticks) within peptide 513 to 522 (dark blue) through the (green) connector. The influence on the catalytic triad (light blue sticks) is seen through the (green $\beta$-sheet) connector between peptide 533-550 (purple) and Tyr560 (yellow). FXIII monomer A is (black) and monomer B is (silver). Image constructed utilizing VMD (51).

As mentioned previously, the FXIII segment $513-522$ exhibits a decrease in deuteration in the presence of $1 \mathrm{mM} \mathrm{Ca}^{2+}$ and no change is observed for the segment 533-550. These results led to an interest in monitoring FXIII conformational dynamics after incubation in an environment of $2-50 \mathrm{mM} \mathrm{Ca}^{2+}$ at physiological $37^{\circ} \mathrm{C}$. Curiously, the protective effect on $513-522$ was lost and replaced with a steady increase in the exposure of 533-550. In the X-ray crystals, the FXIII active site is occluded by Y560 (Figure 11 yellow sticks) which resides only 10 residues from 533-550. Steady increases in $\mathrm{Ca}^{2+}$ concentration 
might promote movement of $\mathrm{Y} 560$ thus assisting C314 exposure to substrate. Xray crystallographers have previously predicted that $\beta$-barrel 1 must move somewhat away from the catalytic core during the activation process (42). Moreover, Y560 will need to be displaced from its $\mathrm{H}$-bonding interaction with catalytic C314. Since the active site region becomes more exposed at higher $\mathrm{Ca}^{2+}$ levels, it is possible the FXIII $533-550$ segment contributes a line of communication to this process. In further support of this proposal, Andersen and Faber observed their own increases in HDX based solvent exposure for FXIII peptides spanning 533-551, 556-559, and 560-573 upon formation of $\mathrm{FXIII}$ (46).

\section{Metal Influence on Dimer Interface}

The ion binding helix has direct interactions with the FXIII $A_{2}$ dimer interface. According to surface calculations, the dimer interface covers $2280 \AA^{2}$ and there is a $10.3-14.4 \mathrm{kcal} / \mathrm{mol}$ decrease in FXIII-A free energy upon

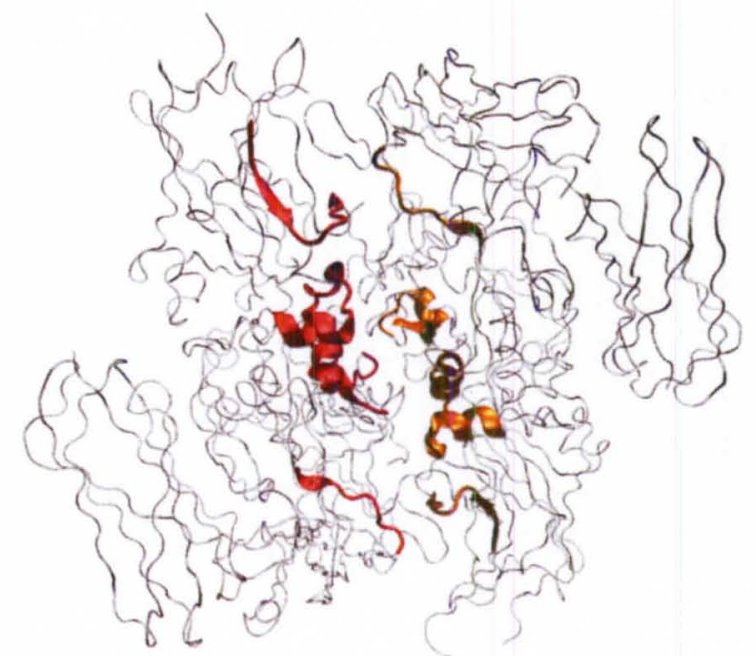

Figure 12: An illustration of all peptic peptides which yield sequence coverage along the dimer interface (100-111, 240-265 and 407-424) are labeled (red) for FXIII monomer A and (gold) for monomer B. Image constructed utilizing VMD (51). 
dimerization $(36,42)$. Key interactions between the two FXIII-A monomers are found between K113-D367', K257-E401' and R260-D404'. See Figure 12 for HDX coverage of the dimer interface. The exposure reported previously (43) around the dimer interface for peptide $240-247$ is also seen in the presence of just $1 \mathrm{mM} \mathrm{Ca}^{2+}$. This dimer interface exposure is further supported by increases in deuterium incorporation now recorded for 248-264 and 248-265. Solvent exposure around the dimer interface is also observed with $1 \mathrm{mM} \mathrm{Ba}^{2+}$ and $\mathrm{Cu}^{2+}$ but to a lesser extent by $\mathrm{Mg}^{2+}$. Although $100-111$ is also positioned along the dimer interface, this peptide segment exhibits protection from deuterium exchange. Only the most C-terminal portion is actually along the dimer interface. The remaining segment is directed into the $\beta$-sandwich region which exhibits protection. These results indicate that a series of different divalent ions can disturb interactions between the two FXIII-A monomers. Such events occur even at $\mathrm{Ca}^{2+}$ levels insufficient to support FXIII activation.

Further increases in solvent accessibility along the $A_{2}$ dimer interface (aa 240-264) could be observed when the FXIII was nonproteolytically activated at $37^{\circ} \mathrm{C}$ with $50 \mathrm{mM} \mathrm{Ca}^{2+}\left(\mathrm{FXIII}{ }^{\mathrm{Ca}}\right)$ or $500 \mathrm{mM} \mathrm{Na}^{+} / 2 \mathrm{mM} \mathrm{Ca}^{2+}\left(\mathrm{FXIII}{ }^{\mathrm{Na}}\right)$. Under different HDX conditions, this same region was seen to increase in accessibility when activated by lla as well as when inhibited by IAA and K9 DON (43). The disruption along the dimer interface due to increasing levels of cations may also be due to the noncanonical $\mathrm{Ca}^{2+}$ binding site reported for TG2 (S1 226-233) which is homologous to $264-271$ found on the dimer interface of FXIII (77). Interestingly, a Yb binding site (Figure 13 green) has been shown by X-ray 
crystallography to be in the vicinity of FXIII residue 271 (42). Yee et al. proposed that an opening in the FXIII $A_{2}$ dimer interface may allow for the lysine acylacceptor to access the catalytic core (27).

\section{Catalytic C314 Region and Substrate Recognition}

The exposure of the catalytic C314 is imperative for FXIII activity. Of the metals tested at $1 \mathrm{mM}, \mathrm{Ca}^{2+}$ promoted the largest increase in solvent accessibility for the FXIII 300-314 region. See orange peptide in Figure $13 . \mathrm{Ba}^{2+}$ exerted a mild increase on this region and $\mathrm{Mg}^{2+}$ remained essentially the same as zymogen. $\mathrm{Cu}^{2+}$ was unique in that it demonstrated substantial protection of 300-314 when compared to zymogen. These HDX results substantiate the lack of TGase activity observed in the presence of $\mathrm{Cu}^{2+}$ and suggest that the observed solvent protection might thwart substrate interactions around the catalytic cysteine.

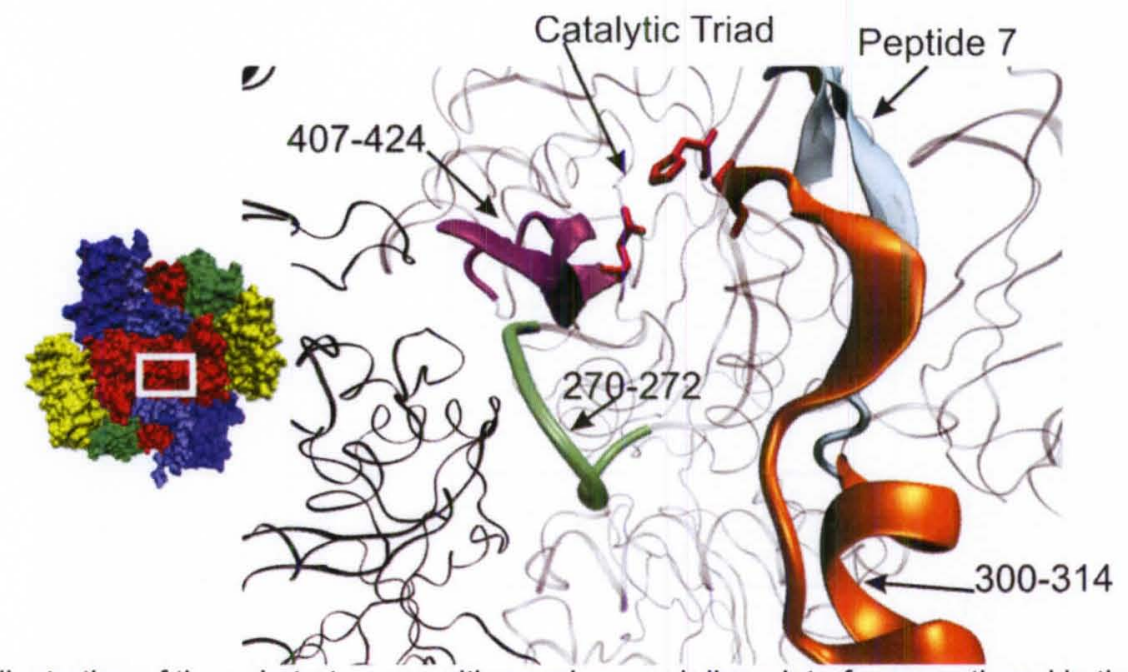

Figure 13: Illustration of the substrate recognition regions and dimer interface mentioned in the conformational dynamics of FXIII $A_{2}$ (1F13). FXIII $A_{2}$ (inset) has each domain labeled: $\beta$-sandwich (blue), catalytic core (red), $\beta$-barrel 1 (green) and $\beta$-barrel 2 (yellow) with the box representing the region of interest. The sequence coverage around the peptide 7 (cyan) the catalytic triad (red) and their close proximity to the dimer interface and the $\mathrm{Yb}$ binding site 270-272 (green). Pepsin peptide 300-314 is (orange) and 407-424 is (magenta). FXIII monomer A is (black) and monomer B is (silver). Image constructed utilizing VMD (51). 
Additional HDX results at physiological temperature revealed that $2 \mathrm{mM}$ $\mathrm{Ca}^{2+}$ had nearly the same effect on exposure of the $300-314$ region as activating FXIII with $50 \mathrm{mM} \mathrm{Ca}^{2+}\left(\mathrm{FXIII}^{\mathrm{Ca}}\right)$. Such results suggest that low $\mathrm{mM} \mathrm{Ca}^{2+}$ contribute to initial exposure of $\mathrm{C} 314$. Further conformational changes likely located within the catalytic core, the dimer interface, and/or $\beta$-barrel 1 must provide the vital link to achieve full FXIII activation and thus ability to target actions at C314.

Interestingly, segment $407-424$ exhibits increased deuteration at $1 \mathrm{mM}$ $\mathrm{Ca}^{2+}$ but loses this solvent exposure when the temperature is raised to $37^{\circ} \mathrm{C}$ and then $2-50 \mathrm{mM} \mathrm{Ca}^{2+}$ is employed. The $407-424$ segment may be influenced by a nearby non-prolyl cis peptide bond Q425-F426 that is in proximity to the dimer interface. Earlier work by our group (60) demonstrated that that FXIII C409 could be alkylated with NEM when the enzyme was nonproteolytically activated with 50 $\mathrm{mM} \mathrm{Ca}^{2+}$ and then re-equilibrated back to $1 \mathrm{mM}$. This ability was, however, lost when the exposed FXIII active site was blocked with an inhibitory Q-containing peptide (43). Weiss and coworkers proposed that conversion of a FXIII nonprolyl cis peptide bond to the more energetically favored trans could help drive larger scale conformational changes to fully expose the FXIII active site region (50). The current increases in $\mathrm{Ca}^{2+}$ concentration and temperature may have aided in establishing an environment that helps promote the next alterations in FXIII conformation. Such an environment may contain a more solvent protected FXIII 407-424 segment. 
Peptide 4 (72-97) in the $\beta$-sandwich and peptide 7 (190-230) in the catalytic core are proposed glutamine substrate recognition regions (71). Peptide 7 is represented by the cyan peptide in Figure 13. FXIII $A_{2}$ in $1 \mathrm{mM} \mathrm{Ca}^{2+}$ displayed enhanced solvent accessibility relative to zymogen for both 88-98 and 214-230. This FXIII exposure around the glutamine recognition region also appears to be $\mathrm{Ca}^{2+}$ specific as it induces the greatest effect compared to the other metals. By contrast, $\mathrm{Cu}^{2+}$ exhibited the smallest amount of exposure and thus appears to be least likely to allow for glutamine recognition. Within peptide 4 , there are differences in solvent exposure depending on the metal tested. In the peptide segment $83-99$, the percent incorporation with $1 \mathrm{mM} \mathrm{Ca}^{2+}$ is essentially the same as with zymogen whereas $\mathrm{Mg}^{2+}, \mathrm{Ba}^{2+}$, and $\mathrm{Cu}^{2+}$ show protection. Within the peptide segment $88-98, \mathrm{Ca}^{2+}$ promoted more exposure than $\mathrm{Mg}^{2+}$ and $\mathrm{Cu}^{2+}$. Another region of interest is the Lysine recognition site 646658 in $\beta$-barrel 2 (72). A peptide located $N$-terminally to this site, $632-646$, is only exposed in the presence of $1 \mathrm{mM} \mathrm{Ca}^{2+}$, whereas $\mathrm{Mg}^{2+}, \mathrm{Ba}^{2+}$ and $\mathrm{Cu}^{2+}$ all display protection in this region.

The proposed glutamine substrate binding site, peptide 7 (190-230), is located in close proximity to the $\mathrm{Ca}^{2+}$ binding site and comes within $3 \AA$ of a loop which connects $\mathrm{D} 438$ to $\mathrm{E} 485$ and $\mathrm{E} 490$ within the $\mathrm{Ca}^{2+}$ binding pocket. For the nonproteolytically activated forms $\mathrm{FXIII}^{\mathrm{Ca}}$ and $\mathrm{FXIIIa}{ }^{\mathrm{Na}}$, the residues $220-230$ exhibited further increases in deuterium incorporation in comparison to the 1-2 $\mathrm{mM} \mathrm{Ca}{ }^{2+}$ conditions. The peptide 7 substrate recognition sequence is another example of a FXIII region that undergoes initial conformational changes at 
physiological $\mathrm{Ca}^{2+}$ levels and then exhibits greater exposure at higher ion

concentrations.

\section{Low mM Metals: Activation Peptide}

The activation peptide (1-37) is cleaved by lla during physiological

activation. Residues 1-23 became protected in the presence of $1 \mathrm{mM} \mathrm{Ca}^{2+}$ as well as $1 \mathrm{mM} \mathrm{Mg}^{2+}, \mathrm{Ba}^{2+}$ and $\mathrm{Cu}^{2+}$. The activation peptide was also protected after nonproteolytic activation with $50 \mathrm{mM} \mathrm{Ca}^{2+}$ at $37^{\circ} \mathrm{C}$, albeit somewhat less than $1 \mathrm{mM} \mathrm{Ca}^{2+}$ at room temperature. Both the FXIII 1-23 and 32-40 segments exhibited protection in the HDX studies.

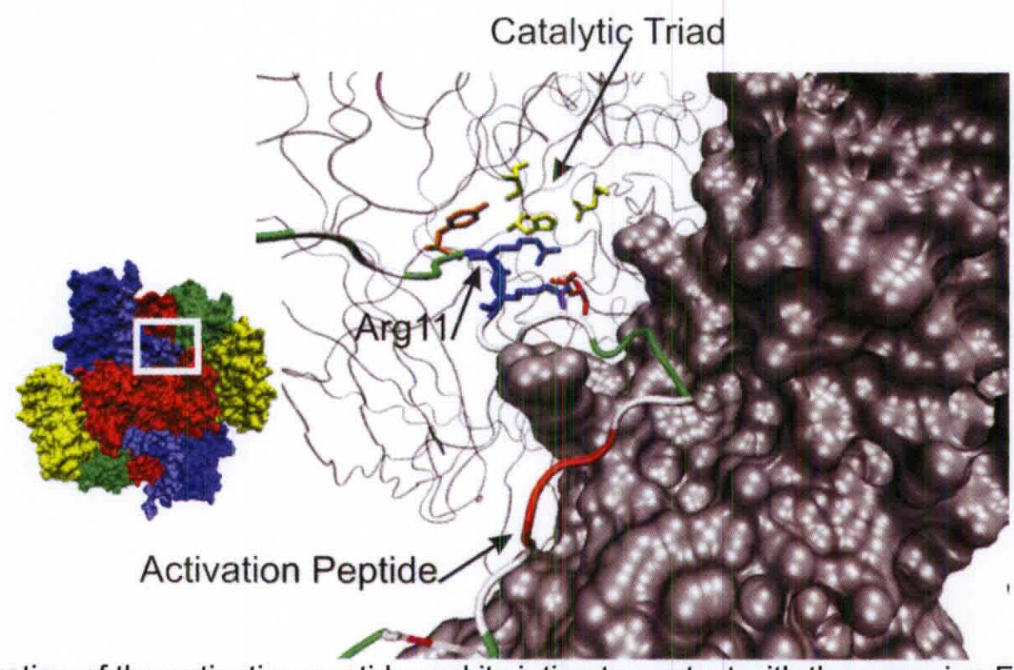

Figure 14: Illustration of the activation peptide and its intimate contact with the opposing FXIII-A monomer (1F13). FXIII $A_{2}$ (inset) has each domain labeled: $\beta$-sandwich (blue), catalytic core (red), $\beta$-barrel 1 (green) and $\beta$-barrel 2 (yellow) with the box representing the region of interest. Depiction of the $\mathrm{N}$-terminal activation peptide of FXIII monomer B (silver surface) labeled according to residue type (green = polar, white = nonpolar, red = acidic and blue = basic) and Arg11 and Arg12 are shown as (blue) sticks. The AP crosses the dimer interface and Asp373' from FXIII monomer A is hydrogen bonded to Arg11 in close proximity to the catalytic triad (yellow) and Tyr560 (orange). Image constructed utilizing VMD (51).

It was reported recently that the FXIII AP is free in solution after lla cleavage and available for binding to monoclonal antibodies specific to FXIII-AP (78). Our HDX results suggest that in the presence of $\mathrm{Ca}^{2+}$ the unhydrolyzed $\mathrm{AP}$ remains tightly associated with FXIII $A_{2}$. Figure 14 shows how the activation 
peptide segment straddles across both monomer units and that this segment contains a combination of polar and nonpolar residues. Moreover, there are acidic and basic side chains that can participate in stabilizing interactions with the FXIII surface. Interestingly, R11 (Figure 14 blue sticks) from the activation peptide segment is hydrogen bonded to D373' on the opposite unit of the dimer. This R11 - D373' interaction is also in close proximity to the FXIII catalytic triad and the vital Y560. Other hydrogen bonds also seem to anchor the FXIII AP above the catalytic core.

Earlier studies by Lewis et al reported that an additional 8 weak metal sites could be found on FXIII at higher $\mathrm{Ca}^{2+}$ concentrations. The AP region and/or a complementary segment on the $\beta$-sandwich domain may accommodate some of these divalent ions (70). Further studies will be needed to assess whether the AP segment may aid in channeling Gln substrates towards the catalytic core. An alternative possibility is that the binding of large physiological substrates will later assist in displacing this cleaved portion of FXIII from the vicinity of the transglutaminase active site.

Further Characterizing Nonproteolytic Activation

Polgar et al. demonstrated that FXIII $A_{2}$ can be activated albeit slowly by $150 \mathrm{mM} \mathrm{NaCl}$ and $2 \mathrm{mM} \mathrm{Ca}^{2+}(40)$. More efficient activation occurs when $\mathrm{NaCl}$ is raised to the $500-1000 \mathrm{mM}$ level. Low calcium, however, is still necessary. In the current work, the organic mono and divalent cations, $\mathrm{TMAC}^{+}$and $\mathrm{EDA}^{2+}$, were investigated for the first time. The $\mathrm{pH}$ of the TGase activity assay is 7.4 and $\mathrm{EDA}^{2+} \mathrm{p} K_{\mathrm{a}_{1}}$ is 7.56 and $\mathrm{p} K_{\mathrm{a}_{2}}$ is 10.71 ensuring a mostly divalent $(60 \%) \mathrm{EDA}^{2+}$ 
ion. As observed with $\mathrm{Na}^{+}, \mathrm{TMAC}^{+}$and $\mathrm{EDA}^{2+}$ each required some calcium to elicit FXIII transglutaminase activity. Curiously, TGase activity for the TMAC $\mathrm{Na}^{+}$, and $\mathrm{EDA}^{2+}$ conditions (all containing $2 \mathrm{mM} \mathrm{Ca}^{2+}$ ) were greater than that of $50 \mathrm{mM} \mathrm{Ca}^{2+}$, our standard nonproteolytic activation method. Such results further emphasize the important role of $\mathrm{Ca}^{2+}$. Moreover, the novel use of organic cations $\mathrm{EDA}^{2+}$ and $\mathrm{TMAC}^{+}$suggests that activation is not only driven by metal ion binding interactions. Unfortunately, ionization issues in the MALDI hindered ability to carry out HDX projects with FXIII $\mathrm{EDA}^{\mathrm{ED}}$ and FXIIIa ${ }^{\text {TMAC }}$. Overview of Results:

In the current project, the FXIII $\mathrm{A}_{2}$ was monitored in the presence of $\mathrm{Ca}^{2+}$, $\mathrm{Mg}^{2+}, \mathrm{Ba}^{2+}, \mathrm{Cu}^{2+}, \mathrm{Na}^{+}, \mathrm{EDA}^{2+}$, and $\mathrm{TMAC}^{+}$with concentrations ranging from 1-2 $\mathrm{mM}$ up to $50-500 \mathrm{mM}$ for nonproteolytic activation. Several regions were found where physiological $\mathrm{Ca}^{2+}$ concentrations initiate the changes needed for activity. As expected, $1 \mathrm{mM} \mathrm{Ca}^{2+}$ does lead to solvent protection for the FXIII region containing the 513-514 secondary cleavage site. Surprisingly, $\mathrm{Ba}^{2+}$ and $\mathrm{Cu}^{2+}$ could protect FXIII 513-522 and 533-550 from solvent even though these two divalent cations do not shield against secondary cleavage (57). By contrast, increasing concentrations of $\mathrm{Ca}^{2+}$ promoted exposure of 533-550. Such $\mathrm{Ca}^{2+}$ induced effects may play a role in exposing the FXIII reactive thiol group by helping to disrupt the $\mathrm{H}$-bond between $\mathrm{C} 314$ and $\mathrm{Y} 560$. When considering other cations, $\mathrm{Mg}^{2+}$ and $\mathrm{Ba}^{2+}$ could both promote nonproteolytic activation in the presence of low $\mathrm{mM} \mathrm{Ca}^{2+}$ whereas $\mathrm{Cu}^{2+}$ could not. The presence of $\mathrm{Cu}^{2+}$ seems to hinder exposure of the active site C 314 as well as the Gln substrate 
recognition region. Our HDX studies also revealed that $1 \mathrm{mM} \mathrm{Ca}^{2+}$ increased deuterium incorporation at the dimer interface, around the lysine recognition region (632-646), and at two glutamine substrate recognition regions, peptide 4 (83-99) and peptide 7 (214-230). These trends continued in nonproteolytically activated FXIII.

There has been much speculation about whether FXIII $A_{2}$ can adopt an open conformation similar to that reported by Pinkas et al. for TG2 (49). Conformational changes to activated FXIII $A_{2}$ can clearly be observed by mass spectrometry approaches. The gross increases in solvent accessibility that would be expected if the $\beta$-barrels had pulled fully away from contacting the catalytic core surface, however, are not observed. The fact that FXIII functions as a dimer may be a contributing element to the difference in behavior of TG2 and FXIII-A $A_{2}$. The dynamic nature of the FXIII in solution is also important to consider.

Calcium appears to play an intriguing regulatory role in FXIII $(33,56,58$, 79). Low $\mathrm{mM}$ levels already begin to initiate important conformational changes needed to expose the active site and make the enzyme ready to accommodate incoming substrates. Often as divalent cation concentration increases, the effects are also enhanced. Physiologically, FXIII is poised to respond to transient influxes of $\mathrm{Ca}^{2+}$ in the presence of a $\mathrm{Na}^{+}$containing environment $(40,52-53)$. Platelet FXIII $A_{2}$ is quite sensitive to such changes in solution environment and can be more readily activated nonproteolytically $(40,52-53)$. The initial conformation events that have been observed in the current study help set the 
stage for the larger conformational changes that are anticipated in the presence of a physiological substrate or inhibitor. 


\author{
CHAPTER III
}

COMPARING THE SOLVENT ACCESSIBILIT OF FXIII-A 2 TO THE OPEN AND CLOSED FORM OF TRANSGLUTAMINASE 2.

\title{
Introduction
}

Very few structural changes have been observed in FXIII when comparing the crystal structures of proteolytically activated and nonproteolytically activated FXIII to zymogen. TG2 on the other hand was recently crystallized bound to an inhibitory peptide substrate in an 'open' conformation $(49,51)$. Comparing these two enzymes in solution will allow for a much better judgment as to whether the

A

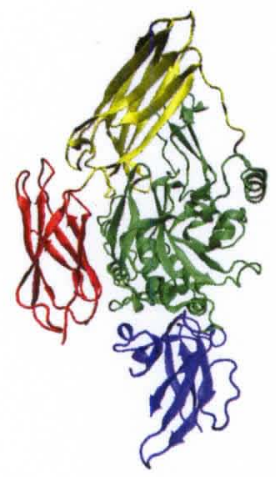

B

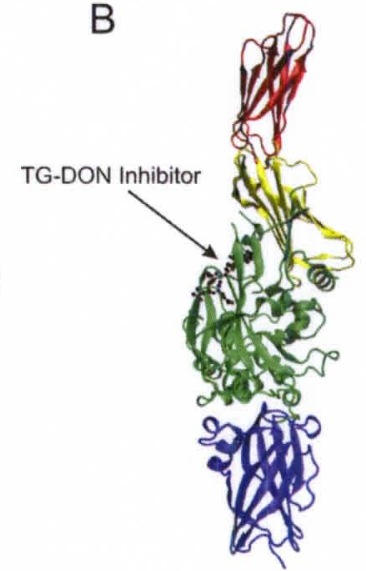

C

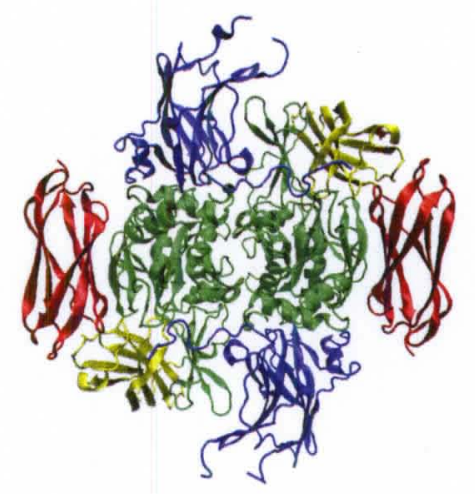

Figure 15: Structure of GDP-Bound and Inhibitor-Bound TG2 and FXIII-A $A_{2}$ Dimer. The Nterminal $\beta$-sandwich is in blue, catalytic core in green, and the C-terminal $\beta$-barrel 1 and $\beta$ barrel 2 in yellow and red respectively. A- GDP-bound (closed) TG2 (1KV3). B- TG2 inhibited (open) with substrate peptide inhibitor Ac-P(DON)LPF-NH ${ }_{2}$ (2Q3Z). C- Factor XIII-A dimer (1F13). 
FXIII-A $A_{2}$ dimer also goes through an 'open' conformation like the TG2 monomer when activated.

Comparing the conformational dynamics of FXIII to that of TG2 offers a unique opportunity. Although TG2 is physiologically a monomer, it contains the same 4 domains ( $\beta$-sandwich, catalytic core, $\beta$-barrel 1 and $\beta$-barrel 2 ) as well as the same general shape as a FXIII-A monomer. As with FXIII, TG2 is proposed to go through a major conformational change during activation or substrate binding, but unlike FXIII, TG2 bound to substrate has now been crystallized in an open conformation (Figure $15(49,80)$ ). TG2 is activated in the presence of millimolar $\mathrm{Ca}^{2+}$ but unlike FXIII it is inhibited by guanine nucleotides. The guanidine nucleotide binding site is located between the catalytic core and $\beta$ barrel 1 . When GTP is bound, the active site is blocked by two loops and the catalytic cysteine ( $\mathrm{C} 277)$ is $\mathrm{H}$-bonded to $\mathrm{Y} 516$ a neighboring tyrosine (81). In the absence of $\mathrm{Ca}^{2+}$, the GTPase activity of TG2 utilizes several G protein-coupled receptors to mediate intracellular signaling $(21,25)$.

The first step in comparing the solvent accessibility of FXIII to that of TG2 is identifying the peptides generated during TG2 pepsin digestion. Pepsin is a non-specific specific enzyme; therefore, it is difficult to predict cleavage products but their cleavage pattern is very reproducible when conducted under the same conditions ( $\mathrm{pH}$, Temp., etc.). Once the peptic peptides were identified and sequence coverage determined, HDX was used to determine the conformational dynamics of TG2 while unactivated (zymogen), activated in the presence of $\mathrm{Ca}^{2+}$ and substrate inhibited. 
Although Hausch was the first to inhibit transglutaminase activity by replacing the substrates reactive glutamine with 6-diazo-5-oxo-L-norlueucine (DON), Sabo et al.was the first to utilize DON to monitor the conformational dynamics of a transglutaminase with substrate bound $(43,82)$. DON is an electrophilic amino acid which acts as an inhibitor by covalently binding to the catalytic cysteine via a thioether linkage (Figure 16). The substrate peptide K9 (Ac-LGPG(DON)SLVIG-OMe) was synthesized and utilized to characterize the A

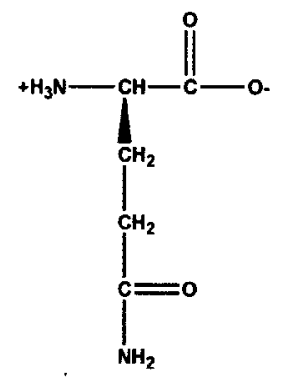

Glutamine

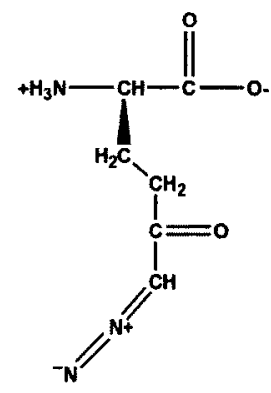

6-diazo-5-oxo-norleucine

B
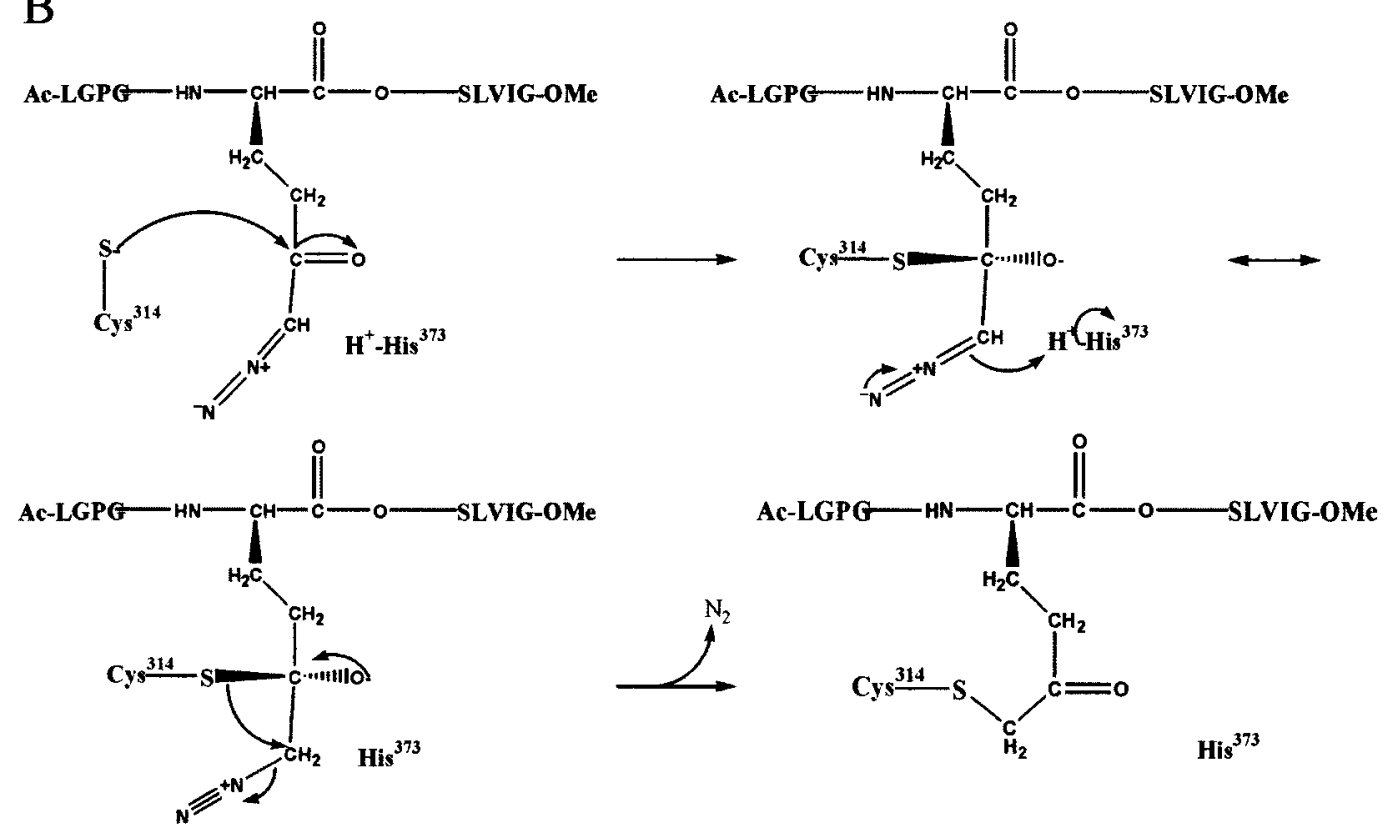

Figure 16: K9 DON Inhibition of FXIlla. A. The isosteres glutamine and 6-diazo-5-oxonorleucine. B. The proposed mechanism of inhibition proceeds through tetrahedral intermediate formation resulting from cysteine attack at the carbonyl group. The methylene carbon then abstracts a proton from the active site H373. Finally, a thioether is formed resulting in the release of $N_{2}$ and irreversible inhibition of $F X I I I a(43,80)$. 
conformational dynamics of FXIII-A $\mathrm{A}_{2}$ when activated and bound to inhibitor (43). HDX was utilized in the current study to monitor TG2 conformational dynamics during activation as a function of calcium concentration and when bound to TGDON, a gluten peptide (Ac-P(DON)LPF-NH ${ }_{2}$ ) inhibitor substrate. Surprisingly there was not a gross increase in deuterium incorporation across TG2. Within the catalytic core, several regions, $451-461$ and $369-377$, became protected when activated and inhibited. The regions $259-269$ and $475-487$ became more exposed only when TG2 was in an open conformation bound to inhibitor substrate. There are antibodies already available that are specific for the open conformation of TG2 and the open conformation is being targeted for pharmaceutical design. The knowledge gained in understanding the regions of TG2 that become more accessible to solvent when in the open conformation will assist in determining whether FXIII- $\mathrm{A}_{2}$ undergoes a similar open conformation that has been reported for TG2 (49). 


\section{Materials and Methods}

\section{Transglutaminase 2 and TG-DON Preparation}

Transglutaminase 2 was purchased from Zedira (Darmstadt, Germany). Lyophilized recombinant human TG2 $(1 \mathrm{mg})$ was reconstituted in $18 \mathrm{M} \Omega$ deionized water, then buffer exchanged into $25 \mathrm{mM}$ Tris-base at $\mathrm{pH}$ 7.4. The concentration of TG2 was determined on a Cary $100 \mathrm{UV} / \mathrm{vis}$ spectrophotometer. The absorbance was monitored at $280 \mathrm{~nm}$ and concentration calculated with the TG2 extinction coefficient of $106,020 \mathrm{M}^{-1} \mathrm{~cm}^{-1}$. The extinction coefficient was determined using Expasy ProtParam tool (http://web.expasy.org/protparam/). Aliquots $(12 \mu \mathrm{l})$ of $50 \mu \mathrm{M} \mathrm{TG} 2$ in $25 \mathrm{mM}$ Tris-base were stored at $-70^{\circ} \mathrm{C}$ until future use.

The gluten derived TG2 inhibitory peptide TG-DON (Ac-P(DON)LPF-NH $\left.{ }_{2}\right)$ was purchased from Zedira (Darmstadt, Germany). The lyophilized powder (10 $\mathrm{mg}$ ) was solubilized in $1.5 \mathrm{ml}$ DMSO and a sample was sent to AAA Service Laboratories (Damascus, OR) for analysis. The TG-DON concentration was determined to be $8.5 \mathrm{mM}$ and stored at $4^{\circ} \mathrm{C}$. Purity was assessed by HPLC and mass spectrometry approaches.

The $\alpha_{2}$ antiplasmin $\left(\alpha_{2}\right.$ AP-Q4N) derived substrate peptide ( ${ }^{1}$ NQENVSPLTLLKLGN ${ }^{15}$ ) was synthesized by SynPep Corp (Dublin, CA). The $\alpha_{2}$ AP peptide was reconstituted in $18 \mathrm{M} \Omega$ deionized water and the concentration (6.8 $\mathrm{mM}$ ) was confirmed by quantitative amino acid analysis (AAA Service Laboratory, Inc., Boring, OR). Purity was assessed by HPLC and mass spectrometry approaches. 
TG2 Transglutaminase Activity Assay

TG2 activity was determined using a modified version of the Dade-Behring Berichrom Assay (62-63). Briefly, this assay utilizes a coupled reaction involving both TG2 and glutamate dehydrogenase (GDH). Activated TG2 reacts with the acyl-donor $\mathrm{a}_{2} \mathrm{AP}-\mathrm{Q} 4 \mathrm{~N}$ releasing $\mathrm{NH}_{3}$ and the transglutaminase reaction concludes when the primary amine acyl-acceptor, glycine ethyl ester, forms an isopeptide bond with the $\alpha_{2} A P-Q 4 N$ peptide. This transglutaminase activity is monitored via the conversion of $\alpha$-ketoglutarate and $\mathrm{NH}_{3}$ into glutamate in the presence of reducing equivalents of $\mathrm{NADH}$. The oxidation of NADH results in decreased absorbance at $340 \mathrm{~nm}$.

TG2 aliquots were diluted to $10 \mu \mathrm{M}$ in $25 \mathrm{mM}$ Tris. For each assay, TG2 $(200 \mathrm{nM})$ was in solution with activator reagent $(163 \mu \mathrm{l})$, detector reagent $(250 \mu \mathrm{l})$ and $\mathrm{CaCl}_{2}(10 \mathrm{mM})$ (64). For monitoring TG-DON inhibition, TG-DON was also added $(0.01-1000 \mathrm{nM})$. The assay contents were placed in a Cary $100 \mathrm{UV} / \mathrm{vis}$ spectrophotometer and incubated for $8 \mathrm{~min}$ at $37^{\circ} \mathrm{C}$ for before the $\alpha_{2} \mathrm{AP}-\mathrm{Q} 4 \mathrm{~N}$ substrate peptide was added. Oxidation of $\mathrm{NADH}$ was monitored for 30 minutes at $340 \mathrm{~nm}$ and the activity was determined by the steepest part of the slope. The slope represents the velocity of the reaction as $\Delta \mathrm{abs} / \mathrm{min}$. TG-DON is solubilized in DMSO, therefore a control experiment was run to confirm that $2 \%$ DMSO did not affect activity of TG2 (data not shown). In previous studies, Factor XIII activity also was not affected with $2 \%$ DMSO (43). HDX Experimentation 
Hydrogen deuterium exchange was utilized to elucidate regions of TG2 that become more accessible to solvent when activated and inhibited. A $12 \mu \mathrm{l}$ aliquot of $50 \mu \mathrm{M}$ TG2 was allowed to come to room temperature. Due to TGDON being solubilized in DMSO, all samples were adjusted to the same

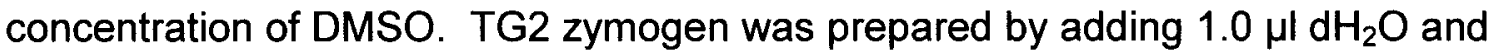
$0.3 \mu \mathrm{l}$ DMSO to a $12 \mu \mathrm{l}$ aliquot of $50 \mu \mathrm{M} \mathrm{TG} 2$ for an assay concentration of $45 \mu \mathrm{M}$ TG-2 in $2 \%$ DMSO. For $\mathrm{Ca}^{2+}$ activated TG2, $1.0 \mu \mathrm{l} 26.6 \mathrm{mM} \mathrm{CaCl}_{2}$ and $0.3 \mu \mathrm{l}$ DMSO were added to a $12 \mu \mathrm{l}$ aliquot of $50 \mu \mathrm{M}$ TG2 for an assay concentration of $45 \mu \mathrm{M} \mathrm{TG} 2$ in $2 \mathrm{mM} \mathrm{Ca}^{2+}$ and $2 \%$ DMSO. Lastly, the activated and inhibited TG2 was prepared by adding $1.0 \mu \mathrm{l} 26.6 \mathrm{mM} \mathrm{CaCl}_{2}$ and $0.3 \mu \mathrm{l} 8.5 \mathrm{mM}$ TG-DON to a $12 \mu \mathrm{l}$ aliquot of $50 \mu \mathrm{M}$ TG2 for an assay concentration of $45 \mu \mathrm{M}$ TG2 in $2 \mathrm{mM}$ $\mathrm{Ca}^{2+}, 191 \mu \mathrm{M}$ TG-DON and $2 \%$ DMSO. Each sample was incubated at $37^{\circ} \mathrm{C}$ for 10 min. then evaporated to dryness in a CentriVap Centrifugal Vacuum Concentrator (Labconco, Kansas City, MO) and placed at $-70^{\circ} \mathrm{C}$ until use.

The following HDX protocol was adapted from methodology established in the Komives laboratory (65-66). The dry FXIII aliquot was allowed to reach room temp before $12 \mu$ of $99.996 \% D_{2} \mathrm{O}$ (Cambridge Isotope Laboratories) was added yielding a final working concentration of $50 \mu \mathrm{M} \mathrm{TG} 2,2.2 \mu \mathrm{M} \mathrm{Ca}^{2+}$ and $213 \mu \mathrm{M}$ TG-DON. The samples were incubated at room temperature for 10 minutes before the HDX was quenched by adding $120 \mu$ l of chilled $0.1 \%$ TFA at pH 2.5 . The quenched reaction was then immediately transferred to a tube containing activated pepsin bound to $6 \%$ agarose (Thermo Scientific, Rockford, IL). Pepsin digestion occurred for 10 min on ice. Following digestion, the reaction mix was 
centrifuged for $30 \mathrm{sec}$ to separate the TG2 digest from the pepsin beads. Three 8.2 $\mu$ l aliquots were immediately frozen in liquid $\mathrm{N}_{2}$ and each reaction condition was performed three times.

In addition to investigating TG2 while inhibited with TG-DON, TG2 was also activated under different concentrations of $\mathrm{Ca}^{2+}(2,5$, and $10 \mathrm{mM})$ in the absence of DMSO, and it was determined that DMSO did not play a role in the conformational changes reported (data not shown).

HDX Analysis

A FXIII(a) HDX aliquot was thawed at room temperature and immediately mixed with an equal volume of $10 \mathrm{mg} / \mathrm{ml}$ a-cyano-hydroxycinnamic acid matrix (a-CHCA) (Aldrich) in 1:1:1 ethanol/ $\mathrm{CH}_{3} \mathrm{CN} / 0.1 \% \mathrm{TFA}$ at $\mathrm{pH} 2.2$, and $0.5 \mu \mathrm{l}$ was spotted on a chilled MALDI plate. The sample spot was then quickly dried by placing the MALDI plate into a SpeedVac. The plate was then immediately inserted into the MALDI-TOF-MS (Voyager DE-Pro, Applied Biosystems). Spectra were collected in reflector mode with 256 shots/spectrum. All peptides in the peptic digest were identified using GPS Explorer ${ }^{\mathrm{TM}}$ after MS/MS analysis on an Applied Biosystems 4700 in the laboratory of M. Merchant at University of Louisville. After digestion by pepsin, the TG2 peptides identified represent $37 \%$ coverage (Figure 17). Peptide identification was confirmed through observing the same peptide sequence in multiple independent digests, with an ion score preferably above 20 (peptic peptides Appendix D). These peptides focus on key TG2 regions found within the $\beta$-sandwich, the catalytic core, and the $\beta$-barrels. Additional studies with acid dependent type XIII protease did not significantly 
1 MAEELVLERCDLELETNGRDHHTADLCREKLVVRRGQPEWLTLHFEGRNYEASVDSLTFS 60

61 VVTGPAPSQEAGTKARFPLRDAVEEGDWTATVVDQQDCTLSLQLTTPANAPIGLYRLSLE 120

121 ASTGYQGSSFVLGHFILLFNAWCPADAVYLDSEEERQEYVLTQQGEIYQGSAKFIKNIPW 180

181 NFGQFEDGILDICLILLDVNPKFLKNAGRDCSRRSSPVYVGRVVSGMVNCNDDQGVLLGR 240

241 WDNNYGDGVSPMSWIGSVDILRRWKNHGCQRVKYGQCWVFAAVACTVLRCLGIPTRVVTN 300

301 YNSAHDQNSNLLIEYFRNEFGEIQGDKSEMIWNFHCWVESWMTRPDLQPGYEGWQALDPT 360

361 PQEKSEGTYCCGPVPVRAIKEGDLSTKYDAPFVFAEVNADVVDWIQQDDGSVHKSINRSL 420

421 IVGLKISTKSVGRDEREDITHTYKYPEGSSEEREAFTRANHINKLAEKEETGMAMRIRVG 480

481 QSMNMGSDEDVFAHITNNTAEEYVCRLLLCARTVSYNGILGPECGTKYLLNLNLEPESEK 540

541 SVPLCILYEKYRDCLTESNLIKVRALLVEPVINSYLLAERDLYLENPEIKIRILGEPKQK 540

601 RKLVAEVSLQNPLPVALEGCTETVEGAGLTEEQKTVEIPDPVEAGEEVKVRMDLLPLHMG 660

661 LHKLVVNEESDKLKAVKGERNVIIGPA 687

Figure 17: TG2 peptides observed by MALDI-TOF-MS after 10 minute pepsin digestion. The magenta area represent peptides observed for TG2 (37\% coverage).

improve sequence coverage. All the MALDI spectra derived from pepsin digests were analyzed using Data Explorer version 4.8(Applied Biosystems) and calibrated using two singly protonated reference peptides; monoisotopic mass 901.44 Da (residues 369-377) and 2042.18 Da + 11 deuterons at mass 2053.29 Da (residues 668-686).

Deuterium incorporation for each isotopic cluster was quantified as described by Sabo et al. (67). To determine the change in deuteration for TG2 under different conditions the percent deuteration for each peptide was calculated using equation 1 (page 23). In accordance with previous HDX data analysis, percent differences greater than $4.5 \%$ are considered significant (45, $67-69), 3-4.5 \%$ is moderate and $<3 \%$ is modest. 


\section{Results}

\section{TG2 Activity and Inhibition with TG-DON}

The kinetics of $\alpha_{2}$ AP as FXIII-A substrate are well established (83). It has also been demonstrated through a Q2N mutation that FXIII will not utilize the GIn in position 4 on the $\alpha_{2}$ AP peptide (83). To determine if TG2 also recognizes only the Gln in position 2, TG2 activity was monitored with $\alpha_{2} A P-Q 2 N$ as substrate. Like FXIII, TG2 was not catalytically active in the presence of $\alpha_{2} A P-Q 2 N$ (data not shown). The activity of TG2 was confirmed using $\alpha_{2} A P-Q 4 N$ as substrate. Following an 8 minute incubation in the presence of $10 \mathrm{mM} \mathrm{Ca}^{2+}$ at $37^{\circ} \mathrm{C}$ the substrate peptide $\alpha_{2} A P-Q 4 N$ was added to the cuvette. The inhibitory action of TG-DON on TG2 activity was determined by adding TG-DON to the cuvette during the 8 minute incubation prior to the addition of substrate. The TG2

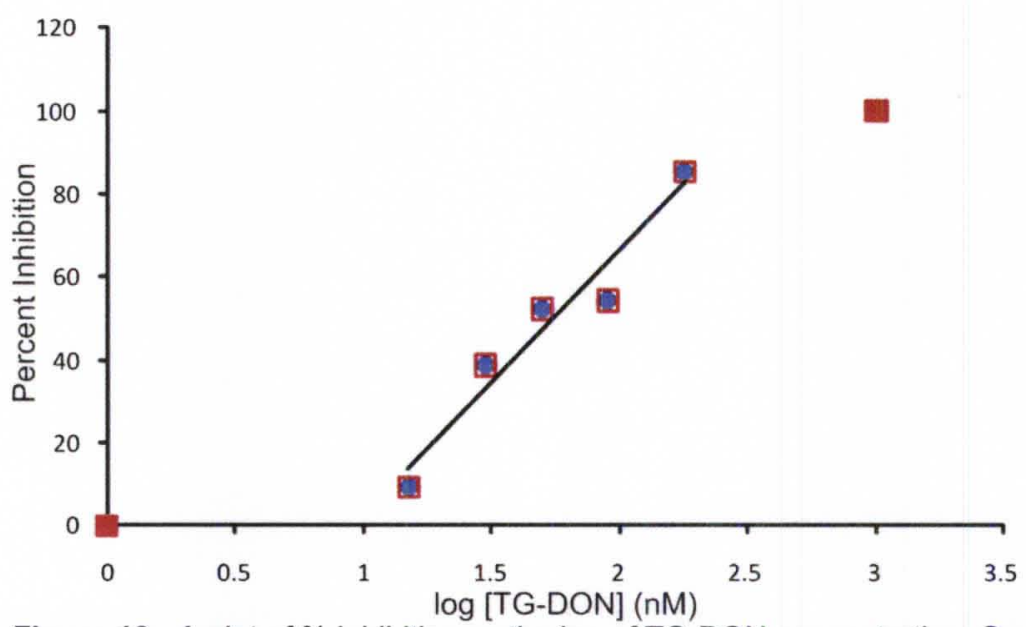

Figure 18: A plot of \% inhibition vs the log of TG-DON concentration. Seven separate trials were performed at varying inhibitor concentrations:TG2 in the absence of TG-DON; 15 nM TGDON; 30 nM TG-DON; 50 nM TG-DON; 90 nM K9 DON; 180 nM K9 DON; and 1000 nM K9 DON. The assay components, TG2, and the indicated amount of TG-DON were incubated for 8 min at $37^{\circ} \mathrm{C}$, at which point the substrate $\alpha_{2} A P-Q 4 N$ was introduced to the assay. All points on the curve are marked with red squares and the linear portion used for determining $I C_{50}=55 \mathrm{nM}$ is blue diamonds inside red squares. 
concentration during activation was $200 \mathrm{nM}$ and TG-DON was monitored over a concentration range of $0-1000 \mathrm{nM}$. By plotting the \% TGase inhibition versus $\log$ TG-DON concentration, a sigmoidal dose response curve is observed (Figure 18). With a TG2 concentration of $200 \mathrm{nM}$, the $\mathrm{IC}_{50}$ was calculated to be $55 \mathrm{nM}$ TG-DON.

MALDI-TOF-TOF for TG2 Pepsin Peptide Identificaiton

The first step in comparing the solvent accessibility of FXIII to that of TG2 was identifying the peptic peptides for TG2. Pepsin is a non-specific specific protease; therefore, it is difficult to predict cleavage products but the cleavage pattern is very reproducible when conducted under the same conditions $(\mathrm{pH}$, Temperature, etc.). Previously in our lab, the peptic peptides of FXIII were determined on an Applied Biosystems DE-PRO using Post-Source Decay (PSD) (45). MALDI-TOF-TOF MS allows for MS/MS capabilities. An Applied Biosystems 4700 was used for acquiring the MS and MS/MS spectra of TG2 digest products. The mass list was subsequently uploaded into GPS Explorer ${ }^{\mathrm{TM}}$ software available through Applied Biosystems. Using GPS Explorer ${ }^{\mathrm{TM}}$ to search the MASCOT ${ }^{\mathrm{TM}}$ database 16 peptides were identified representing $37 \%$ of TG2 (Figure 17 in Material and Methods).

HDX Analysis of TG2

Once TG-DON binding and inhibition were confirmed HDX was utilized to monitor the conformational dynamics of TG2 during activation and in the openinhibited conformation. TG2 has been crystallized in an open conformation leading to the hypothesis that a gross increase in solvent accessibility would be 
Table 3: Changes in Percent Deuteration ${ }^{a}$ : Active TG2 (2 mM Ca2+) and Inhibited TG2 (2 $\mathrm{mM}$ Ca2+ with TG-DON) relative to TG2 zymogen ${ }^{\text {a }}$.

\begin{tabular}{|c|c|c|c|c|}
\hline & Residues & Theo. $D_{\max }^{b}$ & $2 \mathrm{mM} \mathrm{Ca2+}$ & $2 m M$ Ca2+ TG-DON \\
\hline$\beta$-Sandwich & $\begin{array}{l}13-26 \\
27-40 \\
32-40\end{array}$ & $\begin{array}{c}15.8 \\
15.2 \\
9.4\end{array}$ & $\begin{array}{l}-0.3 \\
-0.7 \\
-0.8\end{array}$ & $\begin{array}{l}-0.4 \\
-0.6 \\
-0.4\end{array}$ \\
\hline Cat. Core & $\begin{array}{l}155-161 \\
238-254 \\
260-270 \\
316-330 \\
342-354 \\
370-378 \\
452-462 \\
453-462\end{array}$ & \begin{tabular}{c|}
8.2 \\
18.0 \\
13.0 \\
17.0 \\
12.5 \\
8.0 \\
12.7 \\
11.6
\end{tabular} & $\begin{array}{c}-0.4 \\
-0.4 \\
1.1 \\
0.0 \\
3.3 \\
-4.2 \\
-22.6 \\
-23.2\end{array}$ & $\begin{array}{c}-0.7 \\
0.2 \\
4.8 \\
-0.2 \\
3.6 \\
-4.0 \\
-23.4 \\
-23.7\end{array}$ \\
\hline$\beta$-Barrel 1 & $\begin{array}{l}476-488 \\
503-509 \\
548-555 \\
561-569\end{array}$ & $\begin{array}{c}14.9 \\
8.0 \\
9.4 \\
10.2 \\
\end{array}$ & $\begin{array}{l}0.2 \\
0.4 \\
2.0 \\
0.5\end{array}$ & $\begin{array}{c}5.3 \\
-0.1 \\
2.3 \\
1.1\end{array}$ \\
\hline$\beta$-Barrel 2 & $669-687$ & 20.3 & 6.0 & 8.2 \\
\hline \multicolumn{5}{|c|}{ 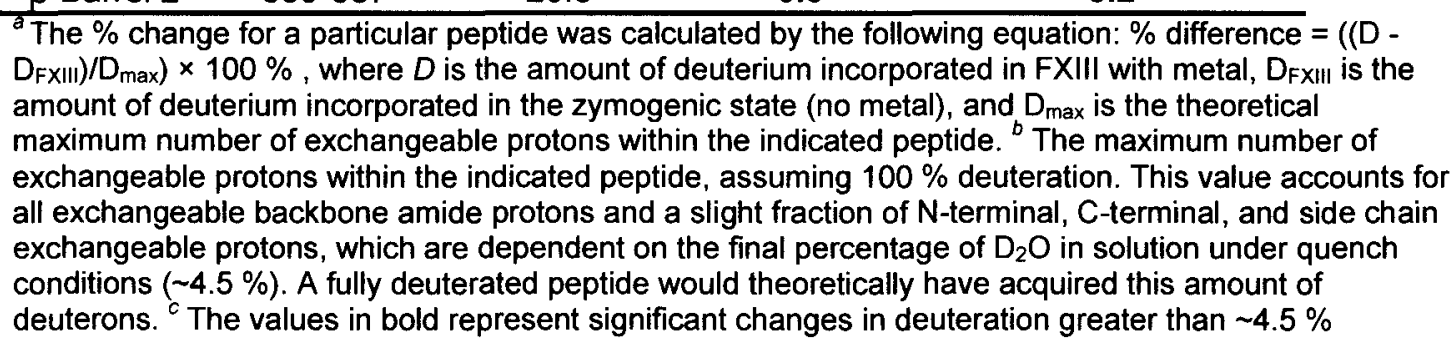 } \\
\hline
\end{tabular}

observed by HDX around the catalytic core and $\beta$-barrel interface. HDX allows for monitoring TG2 conformational changes in solution, eliminating any possible artifacts of the crystallization process. There were four peptides monitored within the $N$-terminal $\beta$-sandwich region, none of which displayed any significant changes in solvent accessibility. The catalytic core was better represented and several peptides displayed not only increases in solvent accessibility, but also protection (Table 3 and Figure 19).

There were 8 peptides analyzed representing $41 \%$ of the TG2 catalytic core $(139-471)$. The majority of the catalytic core did not show any large changes in solvent accessibility. Starting at the $\mathrm{N}$-terminal end of the catalytic 
core, peptide $260-270$ is the first to show a significant change in deuteration.

When compared to zymogen, TG2 activated with $2 \mathrm{mM} \mathrm{Ca}^{2+}$ only displayed a 1.1

$\%$ increase in solvent accessibility whereas the TG-DON inhibited form increased

4.8\%. Continuing toward the C-terminus, the next notable region was 342 - 354 , where both the $\mathrm{Ca}^{2+}$ activated and the TG-DON inhibited conformations had a moderate increase in solvent accessibility, 3.3 and $3.6 \%$ respectively. The next few regions all experienced protection. The first of which is $370-378$ which experienced a -4.2 and $-4.0 \%$ protection for $\mathrm{Ca}^{2+}$ activated and TG-DON inhibited respectively. There were two peptides spanning from 452 - 462 and they both became highly protected when both became protected $22.6-23.7 \%$

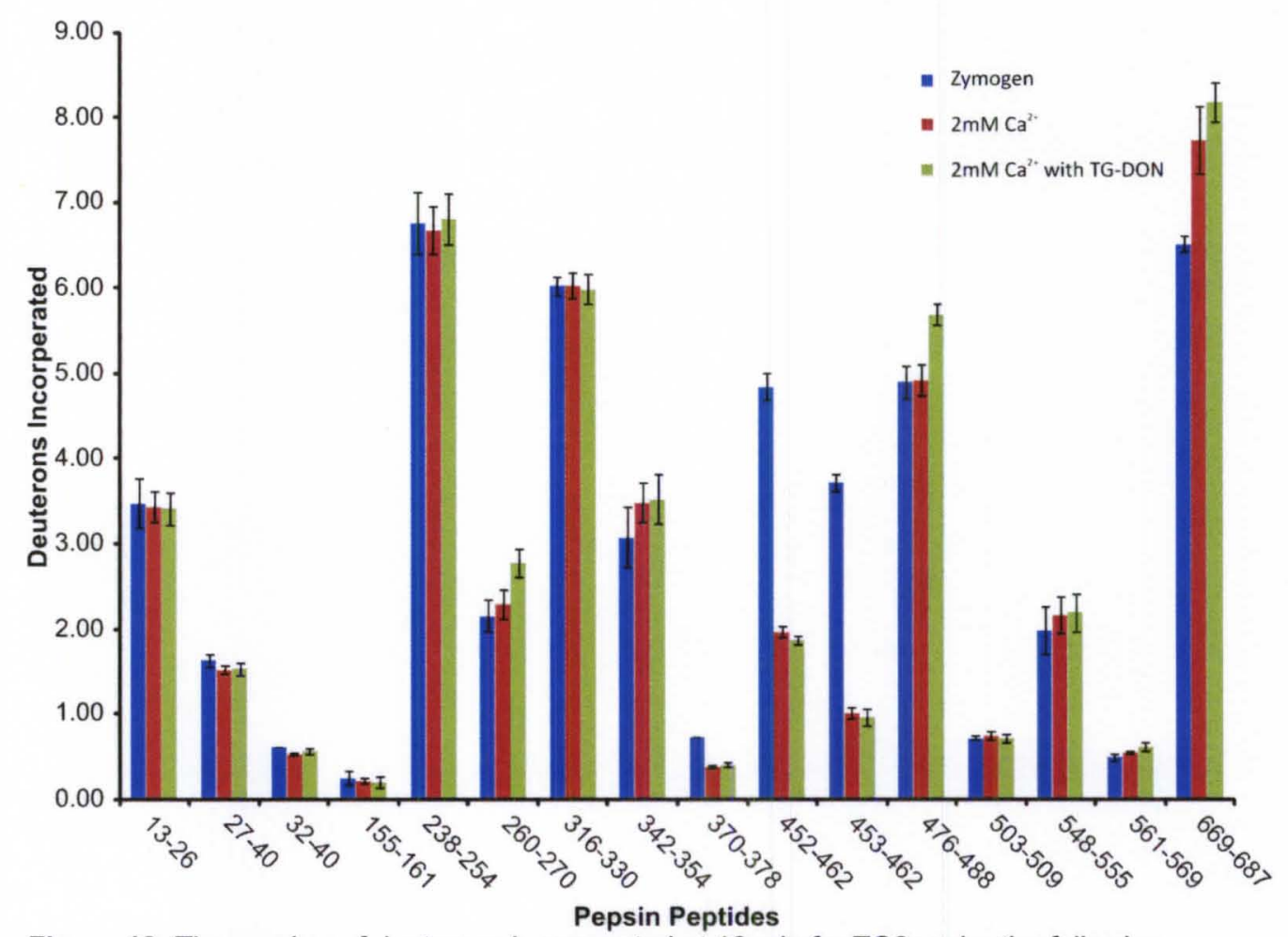

Figure 19: The number of deuterons incorporated at $10 \mathrm{~min}$ for TG2 under the following conditions zymogenic (no metal) TG2 (Blue), $2 \mathrm{mM} \mathrm{Ca}^{2+}$ (Red), $2 \mathrm{mM} \mathrm{Ca}^{2+}$ with TG-DON (Green). Error bars represent the standard deviation of the mean for 3 independent trials.

when activated or inhibited. 
Lastly there are the two $\beta$-barrels ( $\beta$-barrel $1472-584$ and $\beta$-barrel 2585 $-686)$. The 4 peptides that were analyzed in $\beta$-barrel 1 represented $65 \%$ of the 113 amino acids. Only one peptide, 476 - 488 had a significant change in solvent accessibility. Like the $260-270$ region, there was not any notable change in solvent accessibility when activated with $2 \mathrm{mM} \mathrm{Ca}^{2+}$, but there was an increase was observed when inhibited with TG-DON. When inhibited, there was a $5.3 \%$ increase in dueteration, whereas $\mathrm{Ca}^{2+}$ activated TG2 only saw a $0.2 \%$ increase. There was only one peptide (669 - 687) representing $19 \%$ of $\beta$-barrel 2 available for analysis. Peptide 669 - 687 also represented the $19 \mathrm{C}$-terminal amino acids of TG2. This region saw increases in solvent availability in both the $\mathrm{Ca}^{2+}$ activated and TG-DON inhibited TG2. The $\mathrm{Ca}^{2+}$ activated increased $6.0 \%$ and the TG-DON inhibited increased $8.2 \%$ when compared to zymogen. 


\section{Discussion}

Lorand and Graham coined transglutaminase 2 "the bête noir" of the transglutaminase family because it was the first of the TGase family discovered and its physiological role remains elusive (84). Progress in understanding the physiological role of TG2 in apoptosis, cell-matrix interactions and human diseases like Central Nervous System (CNS) neurodegenerative diseases, celiac disease and cancer has been nicely reviewed (84-86). Understanding the function and physiological role of TG2 has also been assisted with a greater understanding of its conformational dynamics $(49,87-89)$.

Two recent studies have utilized mass spectrometry to monitor the conformational dynamics of TG2 $(87,90)$. Greenberg, et al. monitored TG2 activity after lysine modification and determined that acetylation of Lys 444,468 and 663 were essential for inhibition (87). The other study identified a redoxsensitive cysteine triad (Cys 230, 370 and 371 ) which need to be fully reduced for TGase activity (90). Pinkas et al. has crystallized TG2 trapped in an open conformation (49). In the current work, the conformational dynamics of TG2 were monitored in solution, for the first time, through the utilization of HDX coupled with MALDI-TOF-MS.

TG2 HDX

Crystallography has been utilized to investigate the structure of TG2 both in the inhibited (open) and GDP-bound (closed) conformations $(49,91)$. It was apparent that within the $\beta$-sandwich domain there was very little structural variability (Figure 20). This lack of conformational dynamics was supported by 
HDX where there were no regions

within the $\beta$-sandwich that

exhibited significant changes in

solvent accessibility.

Catalytic Core

The catalytic core proved to

be more flexible during activation

and inhibition. In addition to the

catalytic triad (C277, H335 and

D358), it also contains the putative

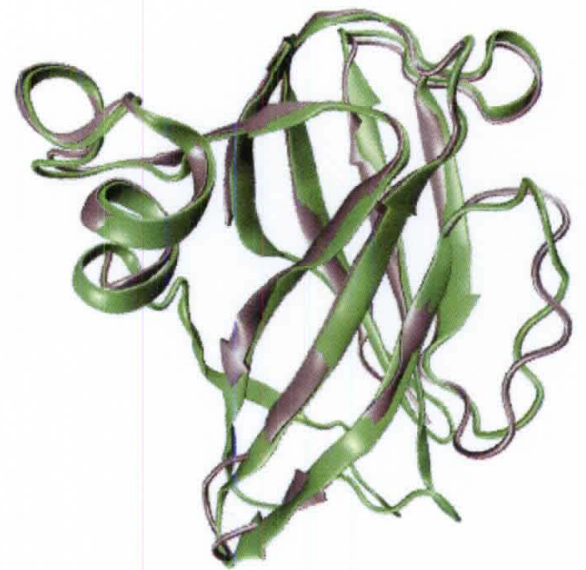

Figure 20: Overlay comparison of the TG2 $\beta$ Sandwich domain in the open and closed conformations. The green represents GDP-bound TG2 in the closed conformation and the grey is the TG-DON inhibited form of TG2. The overlay was performed using the MultiSeq tool in VMD(51).

$\mathrm{Ca}^{2+}$ binding sites, the redox-sensitive cysteine triad (C230, 370 and 371) and two of the three lysines (448 and 468) that are essential for inhibition after acetylation $(77,87,90)$. The exposure experienced from $260-270$ falls $\mathrm{N}$ terminally of the catalytic $\mathrm{C} 277$. During $\mathrm{Ca}^{2+}$ activation the $260-270$ region experienced a modest $(1.1 \%)$ increase in exposure whereas when bound to TGDON, there was a $4.8 \%$ increase in exposure. This region is represented by the magenta helix (Figure 21) and is separated from C277 by a short $\beta$-strand. The 260 - 270 helix does not change orientation in the GDP-bound versus TG-DON inhibited crystal structures; therefore, the differences observed in deuterium incorporation for activated versus TG-DON inhibited TG2 is due to the dislocation of the $\beta$-barrels. The residues of $260-270$ are $<10 \AA$ from residues $631-635$ of $\beta$-barrel 2 in the closed conformation. The movement of the two $\beta$-barrel 
domains during inhibition allows for the $4.8 \%$ increase in deuterium

incorporation.
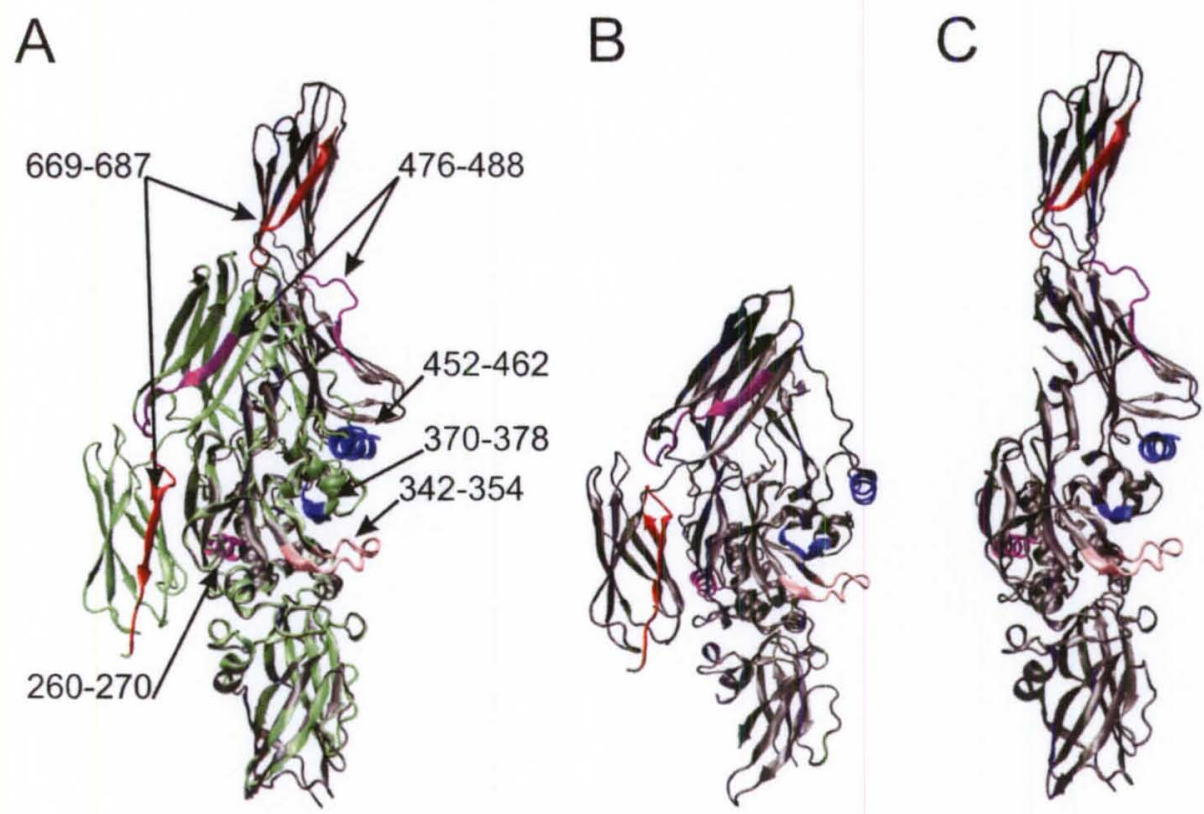

Figure 21: Overlay comparison of TG2 in the open and closed conformations. (A) The green represents GDP-bound TG2 in the closed conformation and the grey is the TG-DON inhibited form of TG2. Regions of interest are labeled according to protection or exposure to solvent after activation and inhibition. Peptides 452-462 and 370-378 (Blue) experienced solvent protection. Peptide 342-354 (pink) experienced moderate exposure. Peptides 260-270 and 476-488 (magenta) experienced exposure only after inhibition. Peptide 669-687 (red) experienced exposure. (B) GDP-bound TG2 in the closed conformation with the same labeling as (A). (C) Inhibited TG2 in the open conformation with the same labeling as (A).

In addition to C277, the catalytic triad also consists of H335 and D358.

Peptide $342-354$ falls between these two residues and $370-378$ falls 12

residues C-terminal of D358. Residues 342 - 354 experienced moderate

exposure when activated and when inhibited (Figure 21 and Figure 22 pink).

This peptide's conformation did not change in the GDP-bound structure when

compared to the TG-DON inhibited crystal structure. These observations

indicate that $342-354$ may become more exposed if the solvent accessibility of GDP-bound TG2 was compared to zymogen. The similar structure of $342-354$ in GDP-bound and TG-DON inhibited TG2 could be due to the importance of 
maintaining structural integrity between two of the three catalytic residues.

Unlike 342 - 354 which became more exposed and displayed a similar conformation in the two TG2 crystal structures, $370-378$ experienced moderate protection and a conformational

change when inhibited. A closer look at this region

demonstrated the intimate contact between $370-378$ and the catalytic D358. In Figure 22, $370-378$ (ball and stick, red $=$ closed and blue $=$ open $)$, the region observed in HDX was viewed with an extension, $355-369$ (new cartoon), which

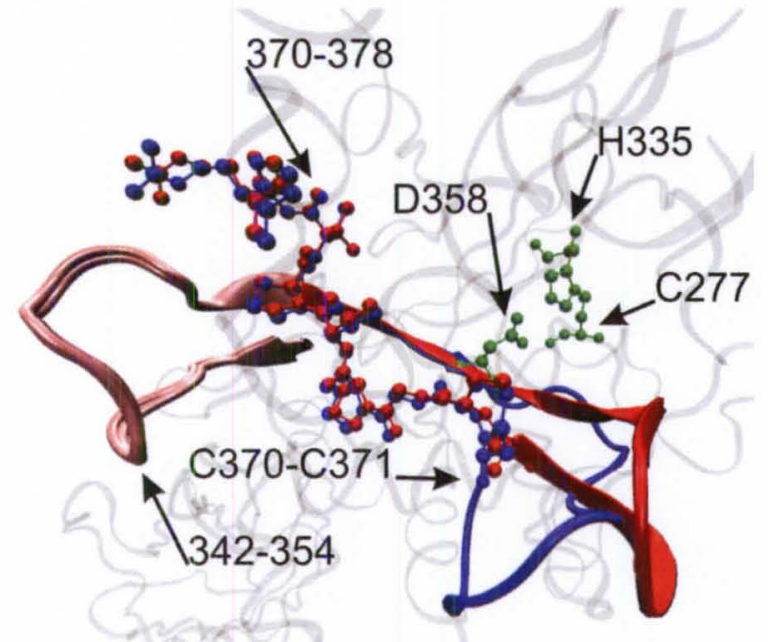

Figure 22: Illustration of the intimate connection between the catalytic triad and peptides $342-354$ and $370-378$. The catalytic triad (C277, H335 and D358) are green ball and stick. Peptide 342-354 (pink) is connected to 370-378 (ball and stick) via a linker containg D358. Peptide 342-354 and the linker are labeled according to conformation (red = closed and blue = open).

contains D358. The beta-hairpin motif on the $\mathrm{N}$-terminus of $370-378$ in the GDP-bound TG2 lost its character and became random coil in the TG-DON inhibited TG2 crystal structure. During HDX, the solvent exposure of $370-378$ decreases 4.2 and $4.0 \%$ for $\mathrm{Ca}^{2+}$ activated and TG-DON inhibited TG2 respectively when compared to zymogen. In the inhibited form of TG2 the Nterminus of $370-378$ underwent a conformational change and lay closer to the surface thus supporting the protection observed by HDX (Figure 22).

There is a disulfide bond reported between C370 and C371 in the open conformation of TG2 $(49,90)$. Stamnaes et al. monitored TG2 under different 
oxidizing (GSH/GSSG) conditions and determined that the vicinal C370 - C371

disulfide was prevalent in the open and oxidized form (90). The solvent protection and observed conformational change at the $\mathrm{N}$-terminus of $370-378$ help to substantiate the evidence of vicinal disulfide formation in the open conformation.

The $\mathrm{Ca}^{2+}$ binding site on TG2 has yet to be observed in crystal structures and has only been identified through modeling and mutations of noncanonical $\mathrm{Ca}^{2+}$ binding regions $(77,88)$. One such proposed $\mathrm{Ca}^{2+}$ binding site $(\mathrm{S} 2$, residues 445 - 455) was identified through sequence homology with the FXIII-A $\mathrm{Ca}^{2+}$ binding site (77). The $\mathrm{HDX}$ of this proposed $\mathrm{Ca}^{2+}$ binding region is

A

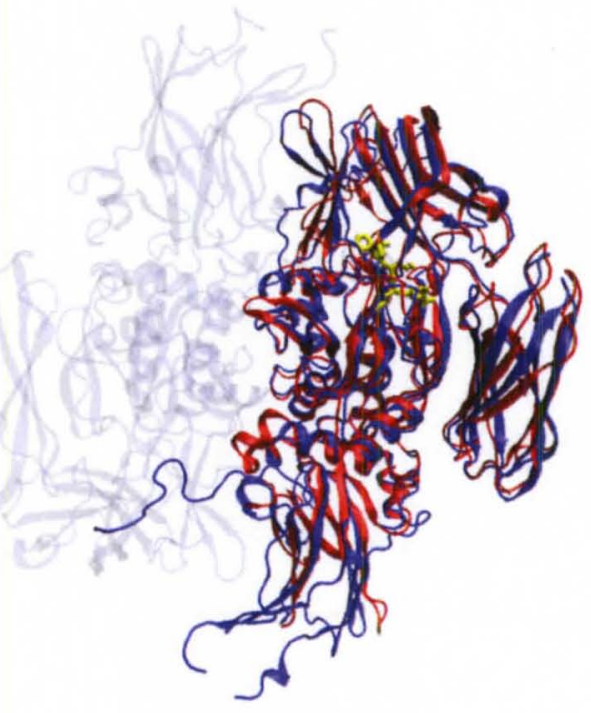

B

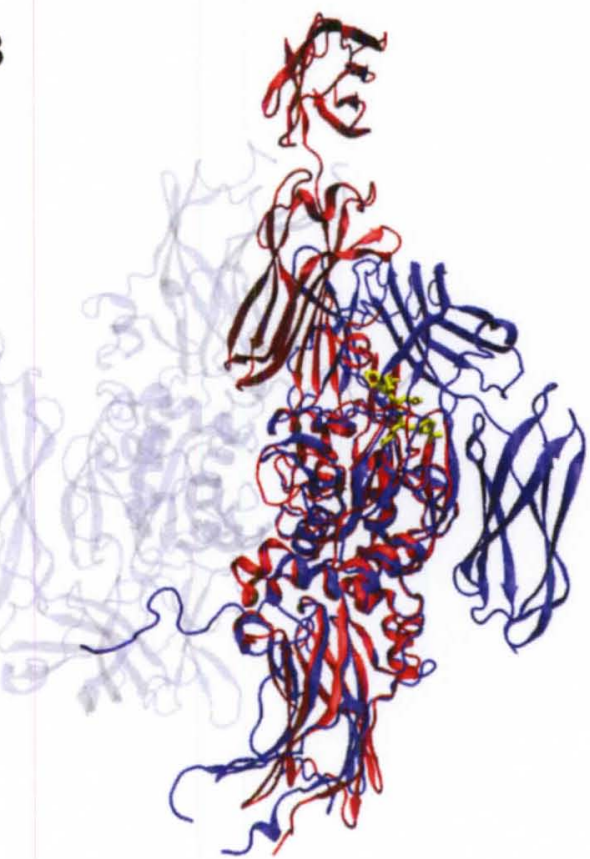

Figure 23; Overlay of TG2 and Factor XIII-A2. (A) The overlay of FXIII-A $\mathrm{A}_{2}$ and the closed conformation of TG2. One monomer of the FXIII-A $A_{2}$ dimer is shaded while the other is (blue). The GDP-bound (closed) TG2 is (red) and the catalytic triad is (yellow sticks). (B) The overlay of FXIII-A $A_{2}$ and the open conformation of TG2. One monomer of FXIII-A $A_{2}$ is shaded while the other is (blue). The TG-DON inhibited conformation of TG2 is overlaid in (red) and the catalytic triad is (yellow sticks). The overlay was performed using the MultiSeq tool in VMD(51). 
represented by $452-462$, an alpha-helix that lay in a flexible region between the catalytic core and $\beta$-barrel 1. The $>20 \%$ protection observed in for $452-462$ supports the evidence of a noncanonical $\mathrm{Ca}^{2+}$ binding site in this region.

In addition to the noncanonical $\mathrm{Ca}^{2+}$ binding site Lai et al. reported that K468 is one of three lysine residues that, when acetylated, inhibit TG2 activity (87). The decrease in TG2 activity after acetylation was attributed to the inability of this flexible region to change conformation and allow for the open conformation. The a-helix character of 452 - 462 in TGDON inhibited TG2 extends Cterminally when compared to GDP-bound TG2. This extension of $\alpha$-helix character would allow for more intra-helix $\mathrm{H}$-bonding thus decreasing the exchangeable protons. The $452-462$ region was represented by two peptides that shared 462 as their Cterminus. Both peptides experienced the same amount protection, further supporting the evidence that the $\mathrm{C}$ -

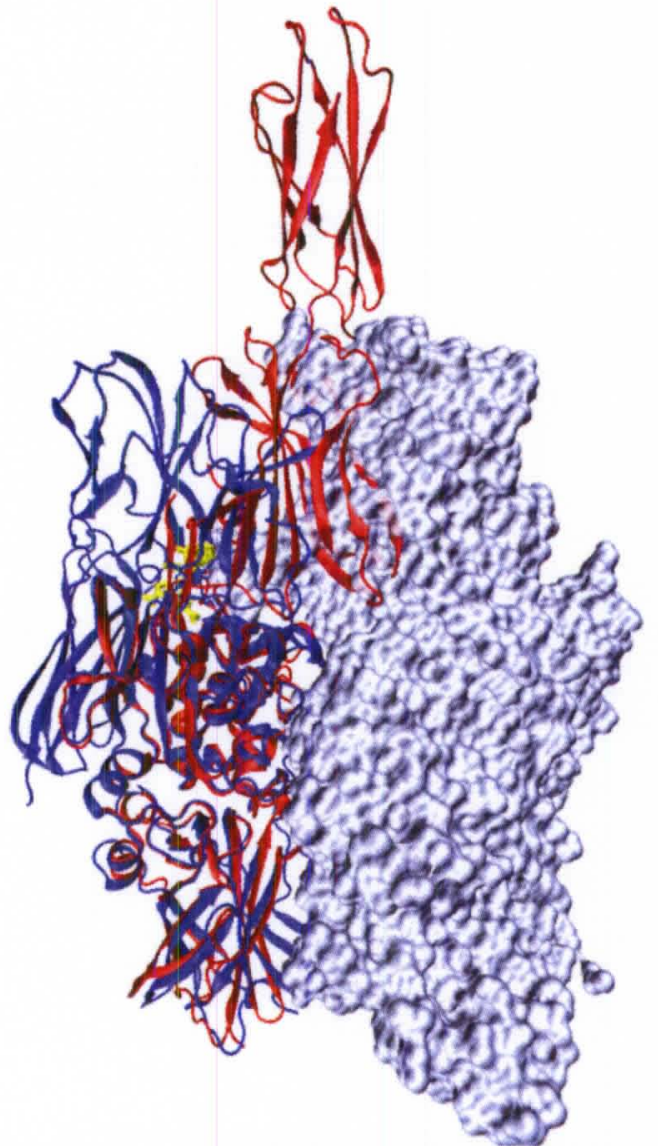

Figure 24: Overlay of FXIII-A $\mathrm{A}_{2}$ and the open conformation of TG2. One FXIII- $A_{2}$ monomer (blue) was aligned with TG-DON (yellow) inhibited TG2 (red). The other FXIII-A $A_{2}$ monomer displays a surface projection emphasizing the interference between the FXIII-A $\beta$-sandwich (surface) and the $\beta$-barrel 1 of TG2 in the open conformation. The overlay was performed using the MultiSeq tool in $\mathrm{VMD}(51)$. 
terminus is important for the observed protection. This helix lies on the backside of the 'hinge' during inhibition (opening) of TG2. Due to this placement it is not surprising that it would become more protected as the TG2 molecule opens.

The conformational dynamics of Factor XIII has been well characterized by HDX and chemical modification techniques $(43-44,46,60,92)$. TG2 and the FXIII-A monomer share a high degree of sequence and structural homology (Figure 23). Due to differences in sequence coverage a direct comparison between TG2 and FXIII HDX was not possible. One region of FXIII which has received lots of attention is the exposure of the dimer interface during activation and inhibition (43-44). The equivalent residues on TG2 were not observed. There has been debate as to whether FXIII-A2 undergoes a large conformational change when inhibited like that reported for TG $(46,49)$. The increase of exposure around the FXIII-A2 dimer interface would seem to support this hypothesis; but, if it is assumed that FXIII-A2 opens in a manner similar to TG2, it would have to overcome substantial steric hinderance between the $\beta$-sandwich region of monomer 1 and the $\beta$-barrel 1 of monomer 2 . When the FXIII-A2 dimer is overlaid on the open conformation of TG2 this blockage between the opposing monomers of FXIII become evident (Figure 24). Komáromi, et al. have attempted to model FXIII-A2 undergoing an open conformation and reported that they had to "eliminate two conformational bumpings by making cuts in the structure and manually deriving new positions for them" (93). Although the data provided to date would not suggest a major conformational change in FXIII-A2 during activation, more work needs to be done to place any certainty on this 
hypothesis. Since 2007 when TG2 was captured in the open conformation there have already been antibodies designed for the detection of the open conformation (Zedira, Darmstadt, Germany). The possibility of trapping FXIII-A2 in a particular conformation via specific antibodies or pharmaceutals would open new avenues for dealing with aberrant coagulation. 
CHAPTER IV

UTILIZATION OF HDX TECHNOLOGY TO ELUCIDATE THE FXIII A RESIDUES CRUCIAL FOR HETEROTETRAMER FORMATION WITH FXIII B.

\section{Introduction}

In plasma, factor XIII is a protransglutaminase with a tetrameric structure consisting of two $A$ subunits and two $B$ subunits $\left(F X I I I-A_{2} B_{2}\right)$, where the $B$ subunits act as a carrier for the catalytically active A subunits. All FXIII-A in plasma is found in the complexed $A_{2} B_{2}$ form, but the $B_{2}$ subunits are in excess and about $50 \%$ are in the uncomplexed $B_{2}$ form (94). To date there is not a crystal structure available for FXIII-B, but by electron microscopy they appear as thin flexible and kinked fibers (95). Factor XIII-B is a glycoprotein which contains $8.5 \%$ carbohydrate and has a molecular mass of $\sim 80 \mathrm{kDa}$. Structurally, the B subunit contains 10 "sushi-domain" repeats (Figure 25), each of which consist of 60 amino acids and 2 disulfide bridges (96-97). 


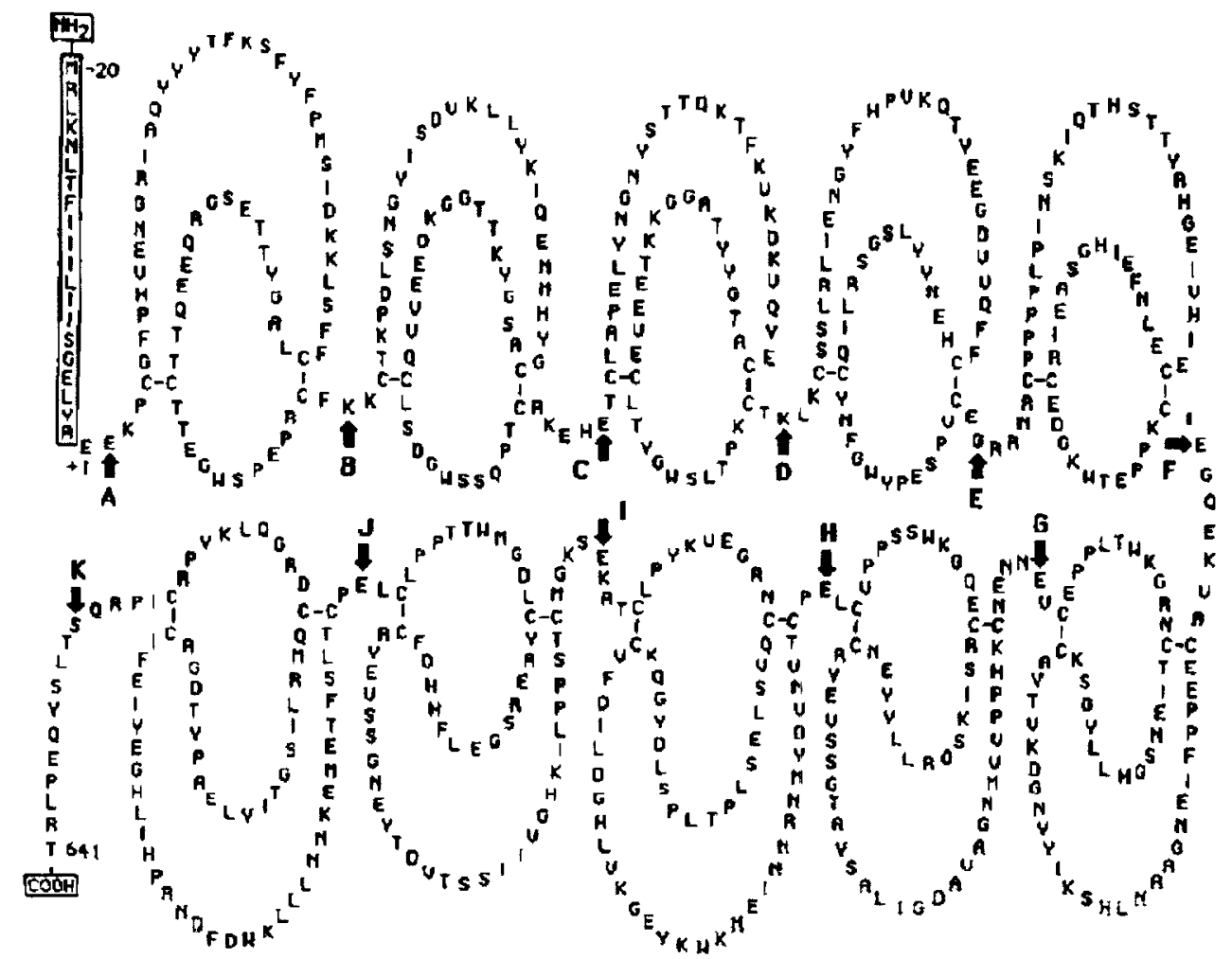

Figure 25: Primary sequence of FXIII $B_{2}$ illustrating the 10 sushi-domain repeats each containing 60 $A A$ and 2 disulfide bonds. This research was originally published in The Journal of Biological Chemistry. Ichinose, A., Bottenus, R.E., Davie, E.W. Structure of Transglutaminases. Journal of Biological Chemistry. 1990; 265:23 13411 - 13414. (C) the American Society for Biochemistry and Molecular Biology (96).

Factor XIII-A exists as the $\sim 320 \mathrm{kDa} \mathrm{A}_{2} \mathrm{~B}_{2}$ heterotetramer with a concentration of $14-28 \mu \mathrm{g} / \mathrm{ml}$ in plasma. The apparent binding constant for the association of $A_{2}$ and $B_{2}$ subunits was determined to be $4 \times 10^{7} M^{-1}(K d=0.025$ $\mu M)(25)$. Souri recently utilized several truncated forms of FXIII-B which contained different regions of the molecule (eg. sushi domains 1-9, 1-5, 1-4, 510 , etc.) to identify the sushi domains responsible for dimer $\left(B_{2}\right)$ formation and heterotetramer $\left(A_{2} B_{2}\right)$ formation. The fourth and ninth domains are crucial for dimer formation and the first sushi domain on the $\mathrm{N}$-terminus is needed for heterotetramer formation (Figure 23) (98). The exact residues on FXIII-B that are important to the $\mathrm{B}_{2}$-dimer and $\mathrm{A}_{2} \mathrm{~B}_{2}$-tetramer formation have not been 


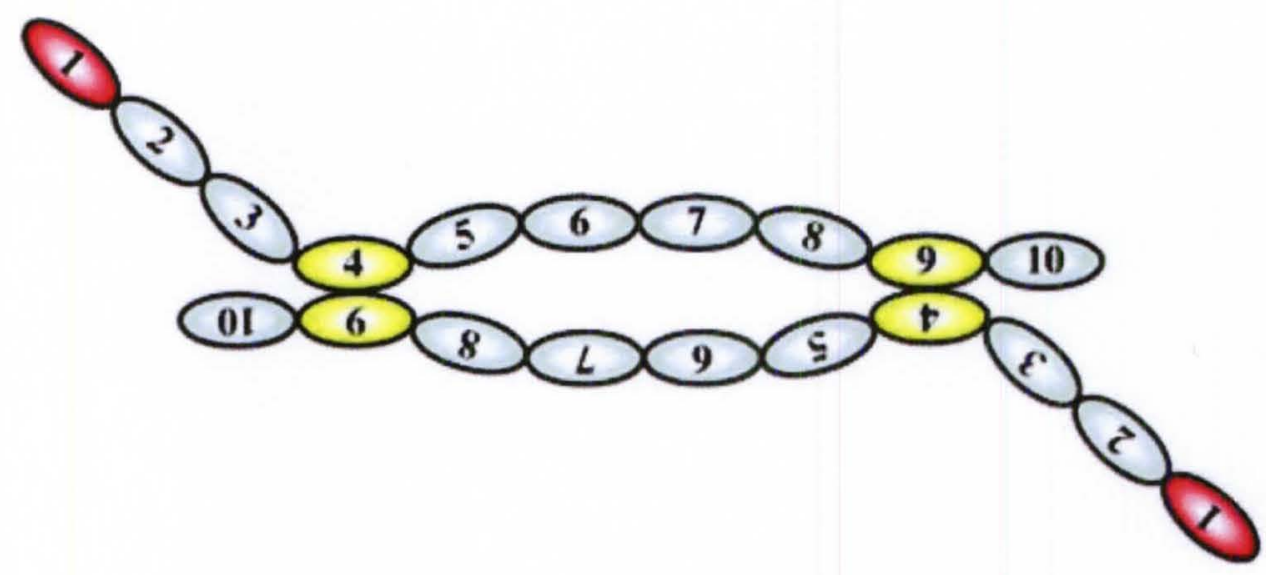

Figure 26: Cartoon model of FXIII B homodimer illustrating sushi-domains 4 and 9 (yellow) which are crucial for FXIII $B_{2}$ dimer formation and domain 1 (red) which is utilized in heterotetramer formation with FXIII $A_{2}$. Figure used from (98).

determined. When complexed with FXIII-B, FXIII-A is stabilized in plasma increasing its half-life. The concentration of FXIII-A decreased $40 \%$ after $2 \mathrm{hr}$ incubation at $37^{\circ} \mathrm{C}$. Further evidence of the stabilization of FXIII-A is the resistance to proteolytic attack by trypsin and thrombin observed when FXIII-A is bound to FXIII-B (99). When bound, the protective effects of the B-subunit are well documented, but the FXIII- $\mathrm{A}_{2}$ residues required for tetramer formation have yet to be elucidated. It is hypothesized that the beta-barrels play a major role in the tetramer formation because when truncated the tetramer is not observed by co-immunoprecipitation with an anti-XIII-B antibody (99).

In the current study hydrogen-deuterium exchange coupled with MALDITOF-MS was utilized to unveil the residues on FXIII-A that become less accessible to solvent when it associates with the $B_{2}$ subunit forming the $A_{2} B_{2}$ heterotetramer. Monitoring FXIII-A solvent protection by HDX aided in painting a more complete picture of how FXIII-A and FXIII-B interact in solution. These experiments demonstrated that although FXIII- $\mathrm{B}_{2}$ incurs $\mathrm{FXIII-A_{2 }}$ proteolytic 
resistance $14 \%$ sequence coverage was still observed. Even with decreased sequence coverage it was demonstrated that much of the FXIII-A molecule becomes protected in the presence of FXIII-B, but significant protection is observed around the dimer interface and both of the beta-barrels. This evidence supports earlier reports of $\beta$-barrel involvement in heterotetramer formation (9899). 


\section{Materials and Methods}

Factor XIII $A_{2}$ and Factor XIII $B_{2}$ Preparation

Recombinant human cellular FXIII $A_{2}$ was generously provided by Dr. Paul Bishop (ZymoGenetics, Inc., Seattle, WA). After reconstituting the lyophilized FXIII in $18 \mathrm{M} \Omega$ deionized water, FXIII was buffer exchanged into $25 \mathrm{mM}$ Trisbase at $\mathrm{pH}$ 7.4. The concentration of FXIII was determined on a Cary $100 \mathrm{UV} / \mathrm{vis}$ spectrophotometer. The absorbance was monitored at $280 \mathrm{~nm}$ and concentration calculated with the FXIII extinction coefficient of $1.49 \mathrm{ml} / \mathrm{mg} \mathrm{cm}$. Aliquots $(12 \mu \mathrm{l})$ of $34 \mu \mathrm{M} \mathrm{FXIII} \mathrm{in} 25 \mathrm{mM}$ Tris-base were stored at $-70^{\circ} \mathrm{C}$ until future use.

A $200 \mu \mathrm{g}$ ampoule of recombinant human FXIII B ${ }_{2}$ (Zedira, Darmstadt, Germany), lyophilized in the presence of $25 \mathrm{mM}$ Tris, was resuspended with 18 $\mathrm{M} \Omega$ deionized water to a concentration of $24 \mu \mathrm{M}$. Aliquots of $10 \mu \mathrm{l}$ were prepared and stored at $-20^{\circ} \mathrm{C}$ until use.

HDX Experimentation

Hydrogen deuterium exchange was utilized to elucidate points of contact between the FXIII $A_{2}$ and FXIII $B_{2}$. An aliquot of $24 \mu \mathrm{M} F$ XIII $B_{2}$ and $34 \mu \mathrm{M}$ FXIIIA $A_{2}$ were allowed to come to room temperature. For analysis of FXIII $A_{2}$ in solution, $6 \mu \mathrm{l} \mathrm{FXIII} \mathrm{was} \mathrm{added} \mathrm{to} 10 \mu \mathrm{l} 25 \mathrm{mM}$ Tris for a final concentration of 13 $\mu \mathrm{M}$ FXIII-A. To allow for FXIII $A_{2}$ and FXIII $B_{2}$ binding before HDX, $6 \mu \mathrm{I} 34 \mu \mathrm{M}$ FXIII $A_{2}$ was added to $10 \mu \mathrm{l} 24 \mu \mathrm{M} F$ XIII $B_{2}$ yielding $13 \mu \mathrm{M}$ FXIII $A$ and $15 \mu \mathrm{M}$ FXIII B in solution. All assays were then allowed to incubate for 20 minutes on ice. Following incubation, the assay tubes were then evaporated to dryness in 
the SpeedVac and then HDX was performed on the dry samples. The following HDX protocol was then adapted from methodology established in the Komives laboratory (65-66). The dry FXIII aliquot was allowed to reach room temp before $12 \mu \mathrm{l}$ of $99.996 \% \mathrm{D}_{2} \mathrm{O}$ (Cambridge Isotope Laboratories) was added yielding a final working concentration of $17 \mu \mathrm{M}$ FXIII A and $20 \mu \mathrm{M}$ FXIII B. The samples were incubated at room temperature for 10 minutes before the HDX was quenched by adding $120 \mu \mathrm{l}$ of chilled $0.1 \%$ TFA at $\mathrm{pH} 2.5$. The quenched reaction was then immediately transferred to a tube containing activated pepsin bound to $6 \%$ agarose (Thermo Scientific, Rockford, IL). Pepsin digestion occurred for $10 \mathrm{~min}$ on ice. Following digestion, the reaction mix was centrifuged for $30 \mathrm{sec}$ to separate the FXIII digest from the pepsin beads. Three $8.2 \mu \mathrm{I}$ aliquots were immediately frozen in liquid $\mathrm{N}_{2}$ and each reaction condition was performed three times.

\section{HDX Analysis}

A FXIII(a) HDX aliquot was thawed at room temperature and immediately mixed with an equal volume of $10 \mathrm{mg} / \mathrm{ml} \mathrm{a-cyano-hydroxycinnamic} \mathrm{acid} \mathrm{matrix}$ (a-CHCA) (Aldrich) in 1:1:1 ethanol/ $\mathrm{CH}_{3} \mathrm{CN} / 0.1 \% \mathrm{TFA}$ at $\mathrm{pH} 2.2$, and $0.5 \mu \mathrm{l}$ was spotted on a chilled MALDI plate. The sample spot was then quickly dried by placing the MALDI plate into a SpeedVac. The plate was then immediately inserted into the MALDI-TOF-MS (Voyager DE-Pro, Applied Biosystems). Spectra were collected in reflector mode with 256 shots/spectrum. All peptides in the peptic digest were previously identified by Brian T. Turner, Jr. (45) and/or 
confirmed by MS/MS analysis on an Applied Biosystems 4700. Peptide identification was confirmed through observing the same peptide sequence in multiple independent digests, with an ion score preferably above 20 (peptic peptides Appendix $C$ ). After digestion by pepsin, the FXIII $A_{2}$ peptides identified represent $40 \%$ coverage. These peptides focus on key FXIII regions found within the $\beta$-sandwich, the catalytic core, and the $\beta$-barrel 1 region. Additional studies with acid dependent type XIII protease did not significantly improve sequence coverage. All the MALDI spectra derived from pepsin digests were analyzed using Data Explorer (Applied Biosystems) and calibrated using two singly protonated reference peptides; monoisotopic mass $850.4787 \mathrm{Da}$ (residues 535-541) and quadraisotopic mass of $1375.7097 \mathrm{Da}$ (residues 220-230).

Deuterium incorporation for each isotopic cluster was quantified as described by Sabo et al. (67). To determine the change in deuteration for FXIII under different conditions the percent deuteration for each peptide was calculated using equation 1 (page 23). In accordance with previous HDX data analysis, percent differences greater than $4.5 \%$ are considered significant ( 45 , $67-69), 3-4.5 \%$ is moderate and $<3 \%$ is modest.

Native Gel

To mimic the HDX conditions, prior to running on the gel, $3 \mu \mathrm{l} 34 \mu \mathrm{M}$ FXIIIA was added to $5 \mu \mathrm{I} 24 \mu \mathrm{M}$ FXIII-B. A $13 \mu \mathrm{M}$ FXIII-A and $15 \mu \mathrm{M}$ FXIII-B aliquot was also prepared and all samples incubated for 20 minutes on ice. Each sample was then added to $16 \mu \mathrm{l}$ sample buffer (63 mM Tris pH6.8, $10 \%$ glycerol and $0.05 \%$ bromophenol blue). The samples were electrophoresed using a 4.0 
$\%$ polyacrylamide stacking gel $(125 \mathrm{mM}$ Tris-base, $\mathrm{pH}=6.8)$ on a $7.5 \%$ polyacrylamide resolving gel ( $375 \mathrm{mM}$ Tris-base, $\mathrm{pH}=8.8)$ and a running buffer of $5 \mathrm{mM}$ Tris Base and $38.4 \mathrm{mM}$ glycine $\mathrm{pH}=8.3$. The gels subsequently were stained with a $0.1 \%$ Coomassie blue solution containing $40 \%$ Methanol and 10 $\%$ Acetic acid. A Gel Doc ${ }^{\mathrm{TM}}$ XR+ System (Bio-Rad, Hercules, CA) was then utilized for gel imaging. 


\section{Results}

\section{FXIII-B induced proteolytic resistance}

All HDX that have been run in our lab to this point have included proteins with a molecular weight of no more than $\sim 160 \mathrm{kDa}(\mathrm{FXIII}-\mathrm{A}=\sim 80 \mathrm{kDa})$. The FXIII- $A_{2} B_{2}$ heterotetramer has a molecular weight of $\sim 320 \mathrm{kDa}(4 \times \sim 80 \mathrm{kDa})$. This increase in total protein mass along with the known proteolytic resistance of FXIII-A in the presence of FXIII-B (98-99) led to an exploratory experiment to determine the feasibility of performing HDX on this system. Following a 10 minute pepsin digest mimicking HDX conditions, peptides produced were
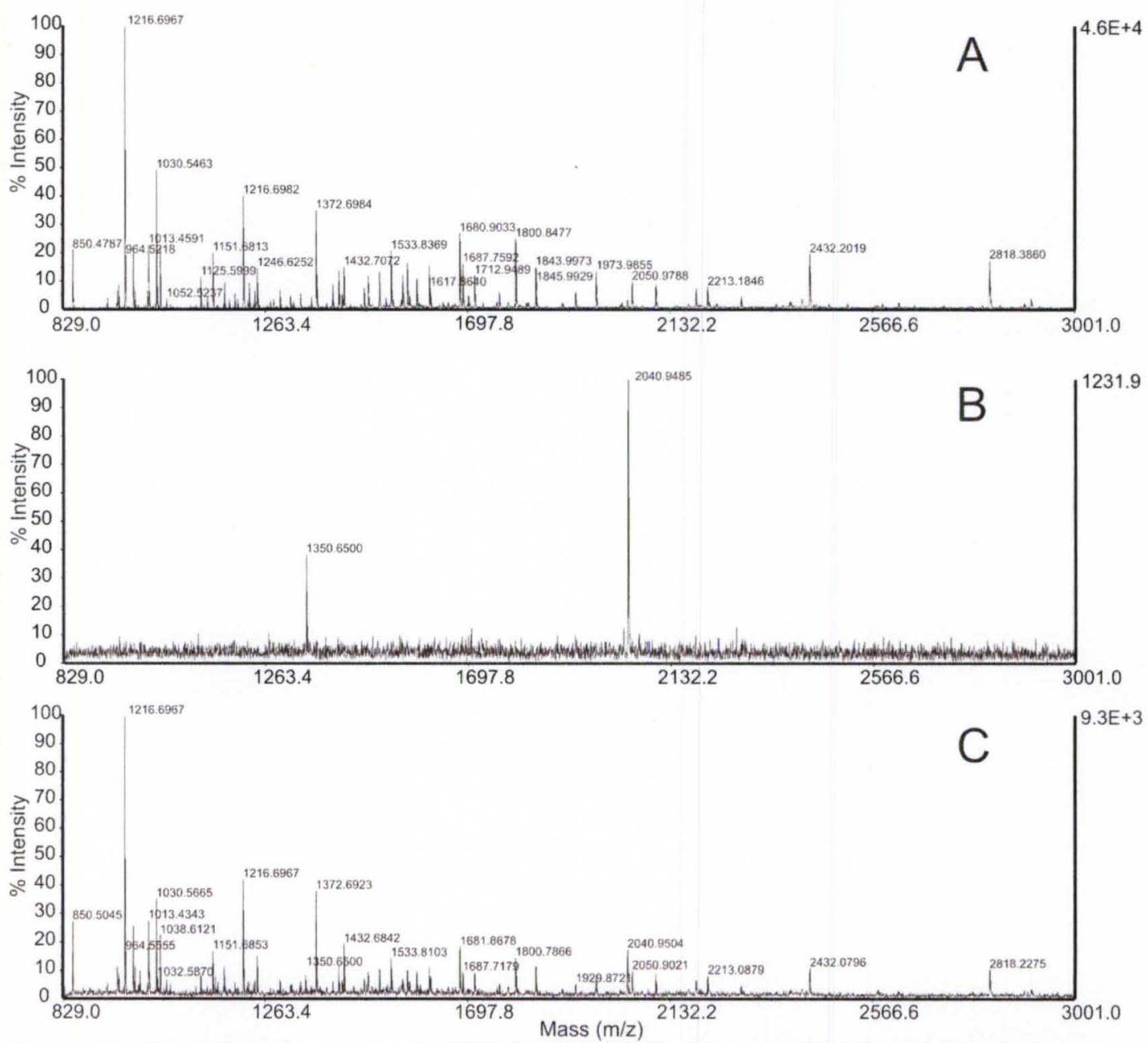

Figure 27: Representative spectra for FXIII- $A_{2}(A), F X I I I-B_{2}(B)$ and $F X I I I-A_{2} B_{2}(C)$ following pepsin digestion. 
monitored for FXIII-A, FXIII-B and FXIII-A $\mathrm{A}_{2} \mathrm{~B}_{2}$ on the MALDI-TOF-MS (Figure

27A-C). At $13 \mu \mathrm{M}, \mathrm{FXIII}-\mathrm{A}$ produced pepsin digest with many clear and

identifiable peaks (Figure 27A). FXIII-B contains 2 disulfide bonds per sushi

domain which infers an innate proteolytic resistance. Due to the proteolytic

resistance, only two major peaks $(\mathrm{m} / \mathrm{z}=1350.65$ and 2039.95$)$ are found in the

FXIII-B pepsin digest (Figure 27B). FXIII-B did elicit proteolytic protection upon

FXIII-A when in the heterotetramer form as seen in Figure $27 \mathrm{C}$. The intensity of

the most abundant ion $(\mathrm{m} / \mathrm{z}=1216.70)$ in Figure 27A is 5 fold higher than that for the spectra representing $13 \mu \mathrm{M}$ FXIII-A in the presence of $15 \mu \mathrm{M}$ FXIII-B.

Suppression within the FXIII- $A_{2} B_{2}$ pepsin digest resulted in a decrease in sequence coverage for the HDX. After digestion by pepsin, the FXIII-A $A_{2}$ peptides identified represent $40 \%$ coverage. These peptides focus on key FXIII regions

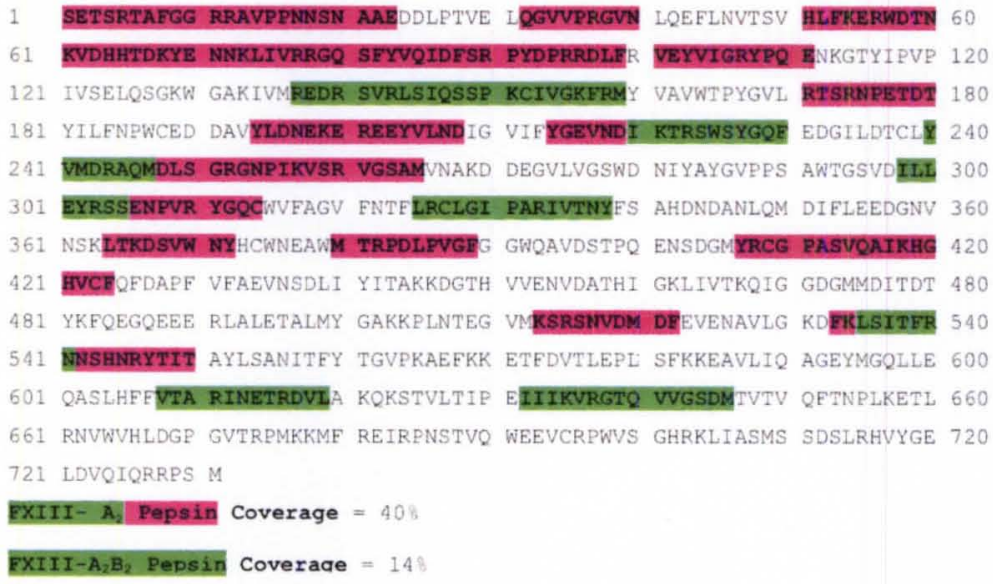

Figure 28: FXIII peptides observed by MALDI-TOF-MS after 10 minute pepsin digestion. The magenta and green represent peptides observed for FXIII-A2 (40\% coverage). The green peptides represent those observed in FXIII-A2B2 digests (14\%). found within the $\beta$ sandwich, the catalytic core, and the $\beta$-barrel 1 region. Although the peaks from the FXIII-A2 digest are observed in the FXIII- $\mathrm{A}_{2} \mathrm{~B}_{2}$ digest, there is a large decrease in sequence 
coverage due to lower sensitivity as well as peak interference (overlap of unidentified peptides). Following pepsin digestion, FXIII-A2 sequence coverage reduces to $14 \%$ when bound to FXIII-B2 (Figure 25).

Native Gel - Heterotetramer confirmation

FXIII- $A_{2}$ has a high affinity for FXIII- $B_{2}(K d=25 n M)$ and is found almost

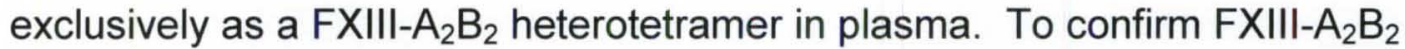
formation for HDX analysis native (no SDS) PAGE was utilized. By mirroring the solution conditions for HDX, native PAGE identified FXIII-A $\mathrm{A}_{2}$ and FXIII- $\mathrm{B}_{2}$ dimers as well as FXIII- $\mathrm{A}_{2} \mathrm{~B}_{2}$ heterotetramer formation in solution (Figure 26). FXIII- $\mathrm{A}_{2}$ migrated the furthest and FXIII- $\mathrm{B}_{2}$ having a similar molecular weight migrated in almost as far as FXIII-A $A_{2}$. The FXIII- $A_{2} B_{2}$ complex was confirmed in the higher molecular weight (slower migrating band).

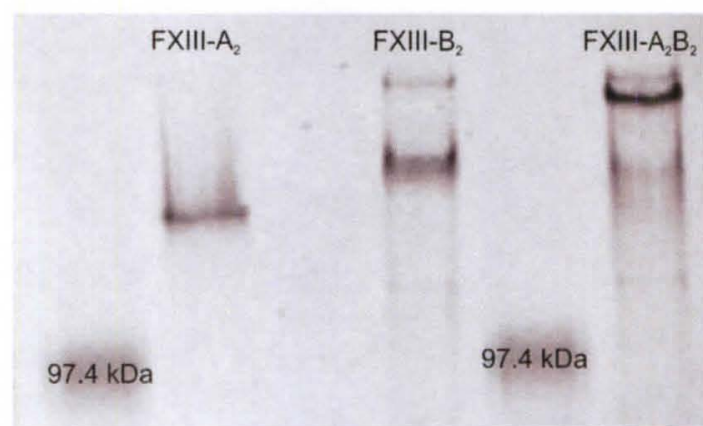

Figure 29: Native PAGE illustrating the formation of FXIII-A $\mathrm{B}_{2}$. Molecular weight standards are marked (97.4 kDa).
After confirming FXIII- $A_{2} B_{2}$ formation, HDX analysis was performed to monitor the regions of FXIII- $\mathrm{A}_{2}$ which become more protected in the presence of FXIII-B $B_{2}$. Due to the interactions between FXIII- $\mathrm{A}_{2}$ and FXIII- $B_{2}$ during heterotetramer formation, there was a substantial

decrease in sequence coverage. Even with this decrease in coverage, regions of FXIII- $\mathrm{A}_{2}$ important for heterotetrmer formation were monitored, for the first time, using HDX coupled with MALDI-TOF MS. 
FXIII- $A_{2} B_{2} H D X$ Analysis

To date FXIII- $A_{2} B_{2}$ has yet to be crystallized; therefore, HDX was utilized in an effort to ascertain the regions of FXIII- $\mathrm{A}_{2}$ that are involved in heterotetramer

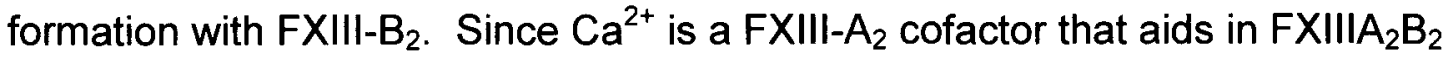
separation and FXIII-A $\mathrm{A}_{2}$ activation, all studies were run in the absence of divalent metal cations $(39,58)$. For the current conformational studies on FXIII $A_{2} B_{2}$ Table 4 displays changes in deuterium incorporation of FXIII-A $A_{2}$ zymogen compared to FXIII- $A_{2} B_{2}$. The total deuterium incorporated for each pepsin-derived peptide is displayed in Figure 30.

The $\beta$-sandwich region of FXIII-A $A_{2}$ contains the activation peptide as well as peptide 4 , a $\mathrm{Gln}$

substrate recognition

region. Due to the

decrease in FXIII-A

sequence coverage, there

were not any quantifiable

peptic peptides within the $\beta$ -

sandwich region. The

catalytic core contains

several regions of interest.

Another important putative

Gln substrate recognition, peptide $7(190-230)$, is
Table 4: Changes in Percent Deuteration: FXIII- $A_{2} B_{2}$ relative to FXIII-A zymogen ${ }^{a}$

\begin{tabular}{lcc}
\hline Residues & Theo. $\mathbf{D}_{\max }{ }^{\mathbf{b}}$ & FXIII- $\mathbf{A}_{\mathbf{2}} \mathbf{B}_{\mathbf{2}}$ \\
\hline $\mathbf{1 3 7 - 1 4 4}$ & 7.90 & -0.8 \\
$\mathbf{1 4 5 - 1 5 9}$ & 15.58 & -1.6 \\
$\mathbf{2 2 0 - 2 3 0}$ & 12.31 & $\mathbf{- 5 . 8}$ \\
$\mathbf{2 4 0 - 2 4 7}$ & 7.50 & $\mathbf{- 1 6 . 7}$ \\
$\mathbf{2 9 8 - 3 0 5}$ & 7.50 & $\mathbf{- 6 . 4}$ \\
$\mathbf{3 2 5 - 3 3 8}$ & 14.40 & 0.9 \\
$\mathbf{3 2 8 - 3 3 8}$ & 9.5 & -2.4 \\
$\mathbf{5 3 5 - 5 4 1}$ & 6.5 & -1.2 \\
$\mathbf{6 0 7 - 6 1 9}$ & 12.80 & $\mathbf{- 1 4 . 5}$ \\
$\mathbf{6 3 2 - 6 4 6}$ & 14.70 & $\mathbf{- 1 0 . 6}$ \\
\hline
\end{tabular}

${ }^{a}$ The $\%$ change for a particular peptide was calculated by the following equation: \% difference $=\left(\left(\mathrm{D}-\mathrm{D}_{\mathrm{FX} I 11}\right) / \mathrm{D}_{\max }\right) \times 100 \%$, where $D$ is the amount of deuterium incorporated in FXIII with metal, $D_{F X I I I}$ is the amount of deuterium incorporated in the zymogenic state (no metal), and $\mathrm{D}_{\max }$ is the theoretical maximum number of exchangeable protons within the indicated peptide. ${ }^{b}$ The maximum number of exchangeable protons within the indicated peptide, assuming $100 \%$ deuteration. This value accounts for all exchangeable backbone amide protons and a slight fraction of $\mathrm{N}$-terminal, $\mathrm{C}$-terminal, and side chain exchangeable protons, which are dependent on the final percentage of $\mathrm{D}_{2} \mathrm{O}$ in solution under quench conditions $(\sim 4.5 \%$ ) A fully deuterated peptide would theoretically have acquired this amount of deuterons. ${ }^{c}$ The values in bold represent significant changes in deuteration greater than $\sim 4.5 \%$ 


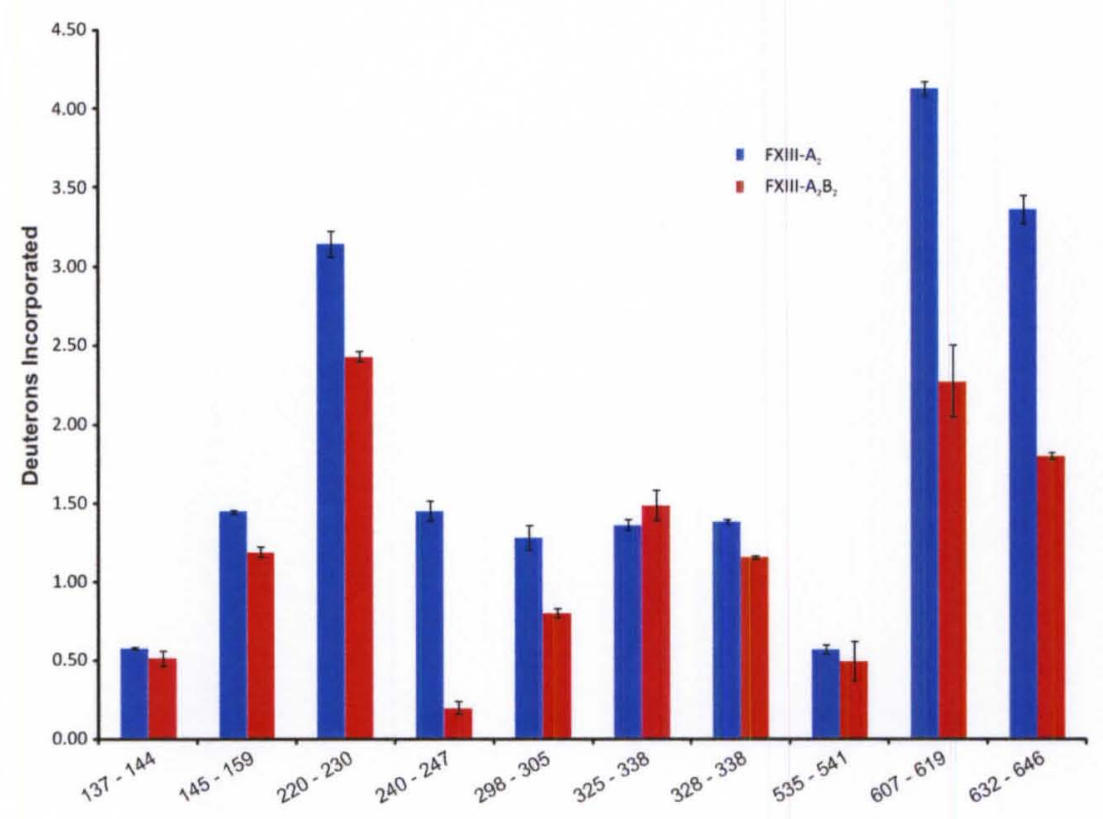

Pepsin Peptides

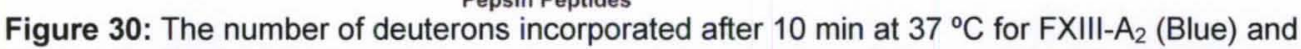
FXIII- $A_{2} B_{2}$ (Red) Error bars represent the standard deviation of the mean for 3 independent trials.

found in the catalytic core(71). In the peptide 7 region, 220-230, FXIII-A experienced significant $5.8 \%$ protection from solvent when bound to FXIII-B. One of the regions which displayed the most protection when bound to FXIII-B was the dimer interface. FXIII-A (240-247), underwent a $16.7 \%$ decrease in solvent accessibility. There were two other regions of the catalytic core represented (298-305) which showed a $6.4 \%$ decrease in solvent accessibility and (325-338) which did not change significantly. FXIII-A peptide 325-338 was the only peptide quantified that displayed an increase $(0.9 \%)$ in HDX exposure. Peptide 328-338 found within 325-338 experienced $2.3 \%$ protection, thus residues 325,326 and/or 327 must be exposed to explain the $0.9 \%$ exposure of 325-338.

The $\beta$-barrels have always been poorly represented during HDX analysis $(43-44,60)$, but they appear to be quite important for FXIII- $A_{2} B_{2}$ heterotetramer 
formation. Protection within the FXIII- $A_{2} \beta$-barrel 1 is evident in the $14.5 \%$ protection at peptide 607-619. This large increase in protection also carries over into $\beta$-barrel 2 where peptide $632-646$, found on the $\mathrm{N}$-terminal end of a proposed Lys recognition region (646-658) (73), is protected $10.6 \%$ when bound to FXIII-B 


\section{Discussion}

Factor XIII has been studied extensively over the past $30+$ years and the knowledge gained is extensive. Ironically very little has been done to determine how FXIII-A and FXIII-B interact. The recent work of Souri et al. has shed new light on the sushi domains that are essential for $F X I I I-B_{2}$ dimer formation as well as FXIII-A $A_{2} B_{2}$ heterotetramer formation (98). The FXIII-A regions necessary for heterotetramer formation have also been investigated and it was determined that the $\beta$-barrels play an integral role (99). These studies have aided in generating a better understanding of FXIII- $A_{2} B_{2}$ formation but there still needs to be more work done to identify the binding epitopes involved on both FXIII-A and FXIII-B.

The preliminary experiments to determine feasibility yielded evidence that FXIII-B is inherently resistant to pepsin proteolysis. After 10 min digestion on ice, FXIII-B only produced two quantifiable peptides $(\mathrm{m} / \mathrm{z}=1350.65$ and 2039.95). This was promising considering that they did not overlap with any of our known peptic peptides produced in FXIII-A. Although the digest containing only FXIII-B did not produce any peptides that overlapped with FXIII-A digests, there were some peaks lost in the FXIII- $A_{2} B_{2}$ digest due to suppression and/or overlap of unidentified peptides. The protective effect of FXIII-B on the proteolytic action of pepsin on FXIII-A was observed in all FXIII- $A_{2} B_{2}$ digests. FXIII-B has been shown to stabilize FXIII-A in vitro (98-99). FXIII-B protected wild type and, to a lesser extent, Y283C FXIII-A from trypsin digestion, but this protection was not seen in a 1464stop mutant which does not contain the $\beta$-barrels (99). A decrease in 
FXIII-A proteolytic degradation due to heterotetramer formation was not only observed with trypsin, but also with the more unspecific proteinase $\mathrm{K}(98)$. The intensity of FXIII- $A_{2} B_{2}$ pepsin digests decreases nearly 5 fold in comparison to FXIII-A $A_{2}$ This signal suppression can be attributed in part to the proteolytic resistance of FXIII- $A_{2}$ when associated with FXIII- $B_{2}$. The protective effect of FXIII-B induced a decrease in digestion efficiency leading to a decrease in FXIIIA sequence coverage from $40 \%$ to $14 \%$ (Figure 31 ).

Once it was determined that HDX was possible with the FXIII- $A_{2} B_{2}$ complex, a native gel was utilized to confirm heterotetramer formation. FXIII- $B_{2}$ was the smallest of the components and thus migrated the furthest. FXIII$\mathrm{A}_{2}$ produced a nice band just above that of FXIII- $\mathrm{B}_{2}$ and heterotetramer formation is confirmed in the slower migrating FXIII$A_{2} B_{2}$ band (Figure 26). $A$

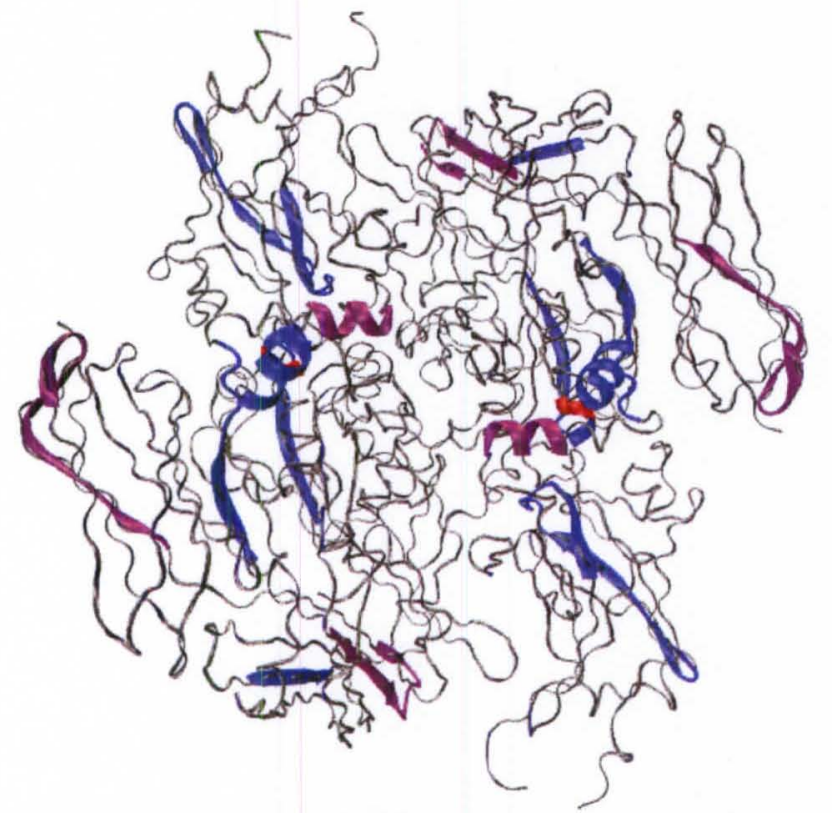

Figure 31: Illustration of FXIII- $A_{2} B_{2} H D X$ sequence coverage. FXIII-A $A_{2}(1 F 13)$ is represented by the ribbons. The cartoon peptides represent $14 \%$ of the molecule. Protection is noted in (blue), significant protection (purple) and exposure (red). Image was constructed utilizing VMD (51)

1:1 ratio of $F X I I I-A_{2}$ to $F X I I I-B_{2}$ has been confirmed in previous studies (9899).Therefore, a faint FXIII- $B_{2}$ band is observed in the FXIII- $A_{2} B_{2}$ lane due to a small excess of FXIII-B $B_{2}(15 \mu \mathrm{M})$ versus FXIII-A $(13 \mu \mathrm{M})$. 
The utilization of

HDX to monitor the

conformational dynamics of

FXIII- $\mathrm{A}_{2}$ when associated

with FXIII-B ${ }_{2}$ allows for

identifying regions that

become protected due to

the heterotetramer

formation. It has been

reported that the $\beta$-barrels

play a crucial role in FXIII-

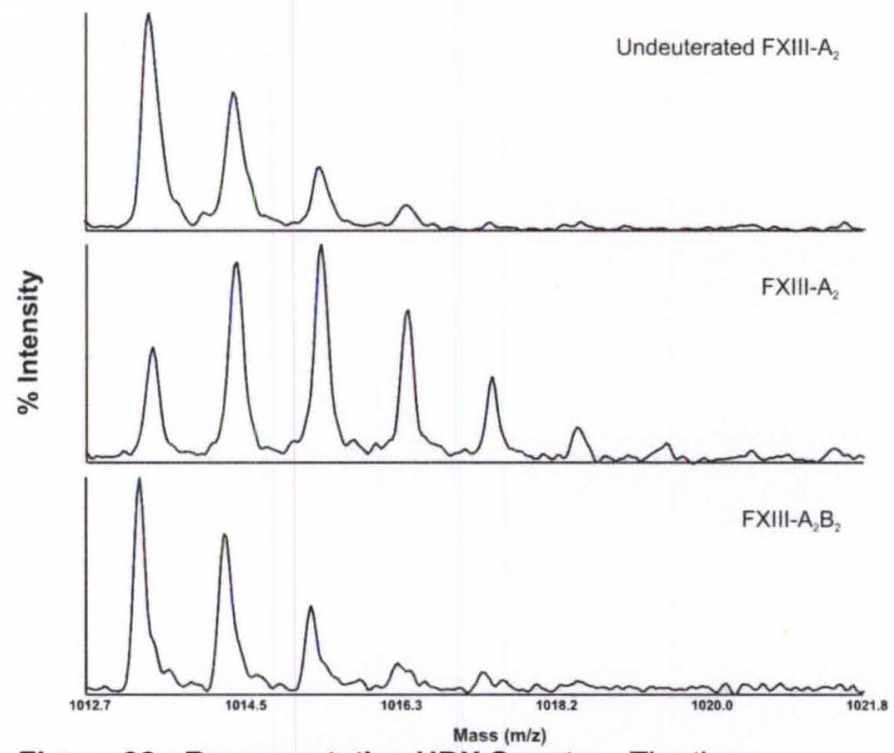

Figure 32: Representative HDX Spectra. The three representative $\mathrm{FXIII-A_{2 }}$ spectra illustrate the isotopic clusters of peptide 240-247 after $10 \mathrm{~min}$. HDX. The FXIII state is listed in the figure. This peak cluster represents a peptide found on the FXIII- $\mathrm{A}_{2}$ dimer interface. Protection due to heterotetramer formation was observed for FXIII- $A_{2} B_{2}$.

$A_{2} B_{2}$ formation (99), but it is unknown as to how the rest of the FXIII-A molecule is affected. The sequence coverage of FXIII- $\mathrm{A}_{2}$ can be seen in Figure 31.

Nearly the whole molecule becomes protected when interacting with FXIII- $\mathrm{B}_{2}$.

Within the catalytic core there were three peptides which underwent significant ( $>4.5 \%$ ) protection. The first regions of importance was the putative GIn binding site, peptide 7 (190-230), represented by peptide $220-230$. This region has been shown to display exposure when activated proteolytically and non-proteolytically, in the presence of physiological concentrations of $\mathrm{Ca}^{2+}$, and when inhibited (43-44). It is thought that FXIII- $A_{2} B_{2}$ is associated with fibrinogen in plasma. By limiting substrate recognition at peptide 7, FXIII-B could be protecting against FXIII-A2 utilizing the fibrinogen as substrate. One peptide falls along the dimer interface (240-247) and it becomes substantially protected in the 
heterotetramer form as seen by the isotopic cluster shift to a lighter mass (Figure 32). The dimer interface becomes more exposed as FXIII-A $A_{2}$ becomes activated so therefore, by remaining protected, FXIII- $\mathrm{B}_{2}$ is limiting the ability of FXIII to aberrantly become activated in plasma. The catalytic cysteine was not represented, but peptide $298-305$ resides 9 residues $\mathrm{N}$-terminally were protected $6.4 \%$ yet again illustrating the protective nature of $F X I I I-B_{2}$ on $F X I I I-A_{2}$.

Prior to the elucidation of the FXIII-A $\mathrm{A}_{2}$ crystal structure, Lai et al. utilized several different C-terminally truncated FXIII-A mutants to define the minimum structure required for transglutaminase activity (56). When truncated at K513 and A502, FXIII-A affinity for small GIn containing substrates only decreases 2 fold, but the turnover rate experiences a 4 fold decrease. This decrease in turnover when the $\beta$-barrels are truncated and complete abolishment of activity when truncated at the $\mathrm{Ca}^{2+}$ binding site demonstrates not only the importance the $\beta$-barrels play in primary amine recognition/turnover, but also the necessity of an intact $\mathrm{Ca}^{2+}$ binding site within the catalytic core (56). Souri et al. has nicely demonstrated the importance of the $\beta$-barrels in heterotetramer formation (9899). FXIII-A 1464stop as well as two mutants that excluded either $\beta$-barrel 1 or $\beta$ barrel 2 were all unable to form FXIII- $A_{2} B_{2}$ heterotetramers. In the present study 607-619 in $\beta$-barrel 1 and 632-646 in $\beta$-barrel 2 experienced 14.5 and $10.6 \%$ protection from solvent when bound to FXIII-B. These results further substantiate the hypothesis that the $\beta$-barrels are essential for heterotetramer formation and give specific residues involved in the FXIII-A $-F X I I I-B_{2}$ interaction. 
It is known that in plasma FXIII- $\mathrm{A}_{2} \mathrm{~B}_{2}$ is largely associated with fibrinogen (100). The binding site of fibrinogen on FXIII-A and/or FXIII-B has yet to be identified. Lai et al. demonstrated that even when truncated at Y481, FXIII-A still binds to fibrinogen even though it has lost $>99 \%$ of FXIIla activity (56). It was proposed that the FXIII-A fibrinogen binding site was independent of the substrate recognition site (71). The protection seen in the $\beta$-barrels would allow for FXIII-A - FXIII-B interactions while still bound to fibrinogen.

Although FXIII-B has not been crystallized, $\beta 2$-glycoprotein I ( $\beta 2$-GPI) contains similar short consensus repeats also referred to as GP-Is or sushi domains.

Topological comparisons

confirmed structural

similarity between the 4 sushi domains of $\beta 2-G P I$ and the sushi domains in 3 other proteins (r.m.s.d $<2.5 \AA$ ) (101). Using the sushi domains of $\beta 2$ -

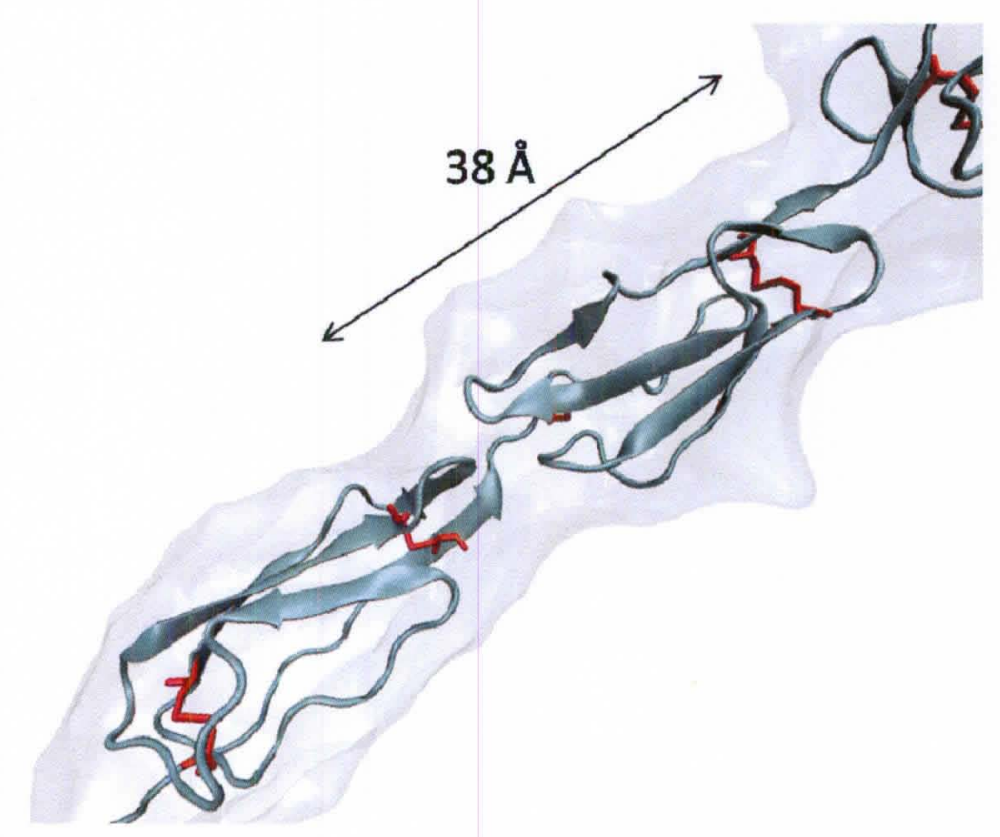

Figure 33: $\beta 2$-glycoprotein I-Representative sushi domain. The two representative sushi domains are illustrated as (cyan) cartoon ribbons with the four cysteines involved in disulfide bond denoted as (red) sticks. Each sushi domain is $\sim 38 \times 17 \times 20 \AA$ so therefore, the 10 FXIII-B sushi domains would span $\sim 380 \AA(101)$.

GPI as a model, it was

determined that a typical sushi domain is $38 \AA \times 20 \AA \times 17 \AA$ (Figure 30 ), therefore, an elongated FXIII-B would be $\sim 380 \AA$ in length (101). The FXIII- $A_{2}$ 
dimer has a length of $\sim 102 \AA$ and each $\beta$-barrel is approximately the same size as a sushi domain (41-42). If FXIII- $B_{2}$ took the orientation proposed by Souri et al. (Figure 30) where the first sushi domain of each FXIII-B was interacting with the $\beta$-barrels and domains 4 and 9 were involved in FXIII- $B_{2}$ dimer interactions, FXIII- $B_{2}$ would stretch $>380 \AA$. Since the FXIII- $A_{2}$ dimer is only $102 \AA$, the FXIII$B_{2}$ dimer would create a D-shaped loop tethered by the first sushi domain to opposing FXIII-A $A_{2} \beta$-barrels. Judging by the HDX results, the FXIII-B loop elicits protection across FXIII- $A_{2}$, but solvent protection is primarily observed at the $\beta$ barrels (the putative FXIII-B2 binding site) and the dimer interface.

HDX has allowed for the first glimpse of the regions of FXIII-A that play

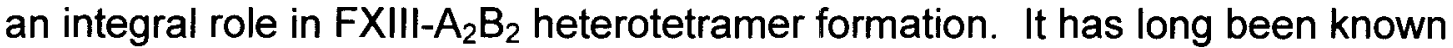
that when bound to FXIII-B,$F X I I I-A_{2}$ is protected from proteolytic degradation. The near global HDX protection observed in FXIII-A in the FXIII- $A_{2} B_{2}$ heterotetramer, brings forth new evidence of the protection bestowed upon FXIII$A_{2}$. The data presented here further emphasizes the importance of the FXIII-A $\beta$-barrels in the interaction between FXIII-A $A_{2}$ and FXIII- $B_{2}$. These studies aid in presenting a clearer picture of $F X I I I-A_{2} B_{2}$ heterotetramer formation. $A$ better understanding of the residues involved in this interaction could potentially be used to promote either the FXIIIA $A_{2} B_{2}$ formation for prevention of aberrant clotting or the release of FXIII-A for pro-coagulant activity. 
Chapter V

DEVELOPMENT OF A FIBRINOGEN ALPHA-C EXPRESSION SYSTEM

UTILIZING MINIMAL MEDIA AND SUBSEQUENT ALPHA-C STRUCTURAL

ANALYSIS VIA NMR

\section{Introduction}

Factor XIII works in concert with thrombin (Ila) and fibrinogen during the last steps of the blood coagulation cascade. Fibrinogen (Figure 34) a $340 \mathrm{kDa}$

A
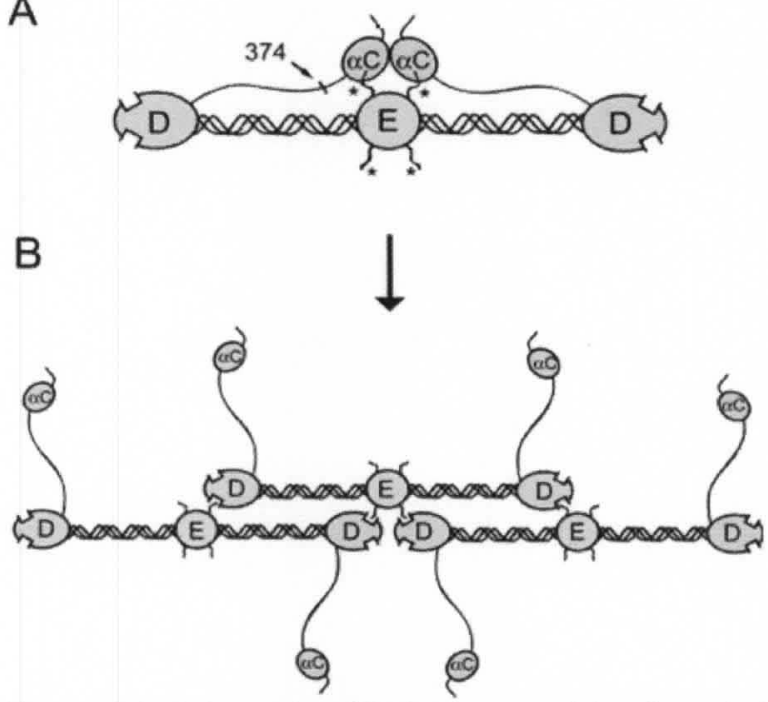

Figure 34: Schematic of fibrionogen and the dissociation of the $\mathrm{aC}$ domain when converted to fibrin(102). A. A Fibrinogen molecule with the D-E-D nodules linked by the coiled coil connectors and the $\mathrm{\alpha C}$ domains interacting with each other and the central $E$ domain via the B peptides. Residue 374 has been labeled to give a point of reference. B. Diagram of a fibrin protofibril where the $\mathrm{aC}$ domains have dissociated after Fbg peptides $A$ and $B$ (marked with

* in A) were cleaved by lla. protein consists of three chains

$(A \alpha, B \beta$ and $y)$ forming the homodimer $(\mathrm{A} \alpha \mathrm{B} \beta \mathrm{Y})_{2}$. The rodlike dimer of trimers has a central E-region which contains the $\mathrm{N}$ termini of the fibrinogen chains.

The two $A \alpha, B \beta$ and $y$ chains extend in opposing directions from the E-region in a coiled coil and terminate in two globular Dregions. Thrombin is responsible for cleaving fibrinopeptides $A$ and

$\mathrm{B}$ (marked with * in Figure $34 \mathrm{~A}$ ) from the $\mathrm{N}$-termini of the $\mathrm{A} \alpha$ and $\mathrm{B} \beta$ chains to form fibrin I and fibrin II respectively (103). The resultant fibrin monomers can align $\mathrm{E}$ and D-regions to form a soft clot. Thrombin, as mentioned previously, is 
also responsible for activating FXIII by cleaving the activation peptide at R37G38. Fibrin acts as a cofactor for the activation of FXIII by decreasing the concentration of $\mathrm{Ca}^{2+}$ needed for FXIII-A $\mathrm{A}_{2}$ to dissociate from FXIII-B $\mathrm{B}_{2}$ and also accelerating the activation peptide cleavage by $1 \mathrm{la}(29,31,74)$.

The C-terminal portion of the Aa chain extends beyond the D-region to form the $\alpha C$-domain (104). The aC-domain (221-610) interacts intramolecularly with each other and the central E-region of fibrinogen until fibrin assembly. During assembly, they dissociate from the E-region and interact intermolecularly promoting lateral aggregation of protofibrils in a process catalyzed by FXIII (105107). The process of aggregation begins with $Y$-chain dimerization of fibrin and is followed by $\alpha$-chain polymerization (108). The sites of intramolecular cross-

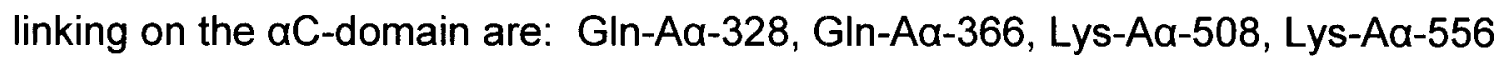
and Lys-Aa-562 (109). There are several crystal structures of fibrinogen, but the $\mathrm{aC}$-domain has been elusive in structural characterization (110-111). The $\mathrm{aC}$ domain, primarily bovine $374-538$ (human equivalent $392-610$ ) has been studied extensively by NMR. These studies have led to the discovery of a $\beta$ hairpin which is held together by a disulfide linkage $\mathrm{C} 423-\mathrm{C} 453$ at its base (102, 112-113). The crosslinking of fibrin monomers by FXIII-A is critical to the formation of stable insoluble clots, yet to date there have not been any studies exploring the structure of the aC-domain in the presence of FXIII-A $\mathrm{A}_{2}$. Fibrinogen $\alpha$ C-domain (233-425) expression 
In order to monitor the conformational dynamics of the fibrinogen $\alpha \mathrm{C}$ -

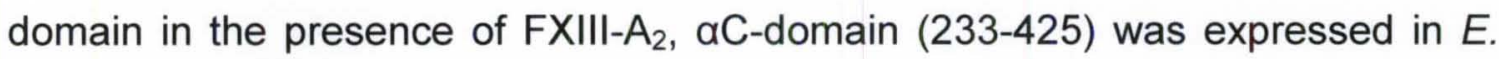
coli. The gene coding for aC (233-425) was cloned into the pGEX-6P-1 vector which has a glutathione-S-transferase (GST) tag as well as ampicillin resistance. There were no mutations made to the primary sequence, so the purified DNA was transformed into DH5a E. coli to create DNA stocks. After collecting DNA via mini prep, the DNA was subsequently transformed into BL21 Gold (DE3) cells for protein expression. The BL21 Gold (DE3) competent cells are the suggested host for pGEX-6P-1 plasmids.

Once $\alpha \mathrm{C}$ expression and purification was confirmed (Figure 35) using conventional methods (LB broth), a method for protein expression was developed utilizing minimal media. Minimal media allows for growth with only the essential nutrients. Providing only the essential nutrients allows for maximizing the incorporation of isotopic labels not found in standard media. To enable future studies using NMR, it was imperative to develop a method of expressing the aC (233425) domain in the presence of isotopically labeled media $\left({ }^{15} \mathrm{~N},{ }^{13} \mathrm{C}\right.$

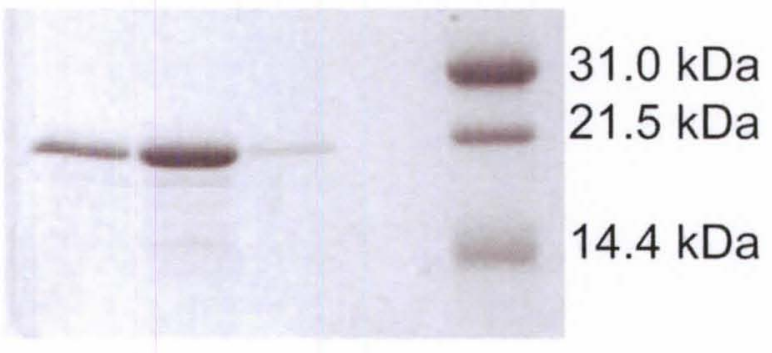

Figure 35: aC Domain (233-425) Purification Confirmation. This is a representative PAGE gel confirming the presence of aC after expression in BL21 Gold cells and purification using GST affinity columns on an AKTA prime system.

and/or ${ }^{2} \mathrm{H}$ ). Due to the high cost of isotopically labeled nutrient sources, there have been several methods designed to maximize growth with limited nutrients (114-116). The aC domain (233-425) was successfully expressed in BL21-Gold 
cells using a method adapted from Marley et. al and Paliy, et. al with minimal media containing ${ }^{15} \mathrm{NH}_{4} \mathrm{Cl}(114-115)$.

NMR methods for analyzing unstructured proteins

Over the past few decades there have been tremendous advancements in NMR technology allowing for protein analysis. NMR has situated itself at the forefront as a method of elucidating protein structure, monitoring molecular dynamics, ligand-receptor interactions, and kinetics (117). To date over 8300 NMR-derived protein structures have been submitted to the Protein Data Bank (PDB). One benefit to NMR analysis of proteins is the ability to gather structural information that is not available through contemporary crystallization techniques. Proteins that do not crystallize well such as membrane bound proteins or proteins that contain flexible regions (118) that are not visible using X-ray technology are of particular interest.

Of particular importance in the initiation of protein structural analysis is the $1 \mathrm{D}{ }^{1} \mathrm{H}$ NMR and the ${ }^{15} \mathrm{~N}-\mathrm{HSQC}$ (Heteronuclear Single-Quantum Correlation) spectrum (119). The $1 \mathrm{D}^{1} \mathrm{H}$ NMR simply displays signals for each proton and the $2 \mathrm{D}{ }^{15} \mathrm{~N}-\mathrm{HSQC}$ shows a unique signal for each proton bound to a ${ }^{15} \mathrm{~N}$. In a $2 \mathrm{D}$ ${ }^{15} \mathrm{~N}-\mathrm{HSQC}$ experiment, the applied magnetization is transferred from any ${ }^{1} \mathrm{H}$ attached to a ${ }^{15} \mathrm{~N}$ nuclei, to the ${ }^{15} \mathrm{~N}$ through scalar coupling (J-coupling). The chemical shift then evolves and is detected after it is transferred back to the ${ }^{1} \mathrm{H}$ (Figure 36). The resulting 2D spectrum contains $a^{15} \mathrm{~N}$ axis and $\mathrm{a}^{1} \mathrm{H}$ axis, and the ${ }^{1} \mathrm{H}$ chemical shifts depend upon the chemical environment (electron density) surrounding it. Therefore each backbone amide (except proline) gives rise to a 


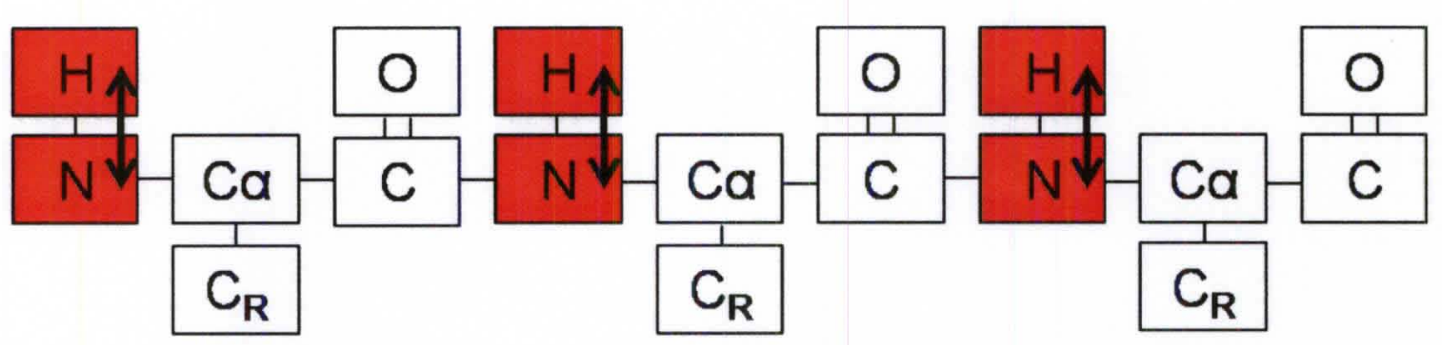

Figure 36: Representative tripeptide displaying the magnetization transfer during a ${ }^{15} \mathrm{~N}-\mathrm{HSQC}$. The amide $\mathrm{N}$ and $\mathrm{H}$ are red to illustrate the atoms involved in the transfer of magnetization. The arrow indicates the travel of energy from the ${ }^{1} \mathrm{H}$ to the ${ }^{15} \mathrm{~N}$ then back to the ${ }^{1} \mathrm{H}$ for detection. The ' $\mathrm{C}_{R}$ ' represents the amino acid side chains.

unique resonance at the ${ }^{15} \mathrm{~N}$ and ${ }^{1} \mathrm{H}$ frequencies for that individual amide group. The more shielded the amide is the more its signal will shift upfield and vice versa. By observing the dispersion of the ${ }^{15} \mathrm{~N}-\mathrm{HSQC}$ chemical shifts, one can estimate whether a protein is unstructured or contains any defined structure.

The aC domain (233-425) contains 2 glutamine residues Gln-Aa-328 and GIn-Aa-366 that are known substrates for FXIII-A2 during blood coagulation. Prior to transglutmainase activity, the $\mathrm{aC}$ domain of fibrin is also known to associate with FXIII-A2B2. The $\mathrm{aC}$ domain is believed to be unstructured in solution therefore eluding crystallography (40-41). The minimal binding domain of hypoxia-inducible transcription factor HIF-1a is an example of an unstructured protein in solution that adopts a structure after it binds, with $\mathrm{nM}$ affinity, to CBP (CREB-binding protein) $(118,120)$. This conformational change in HIF-1a is easily observed through the dispersion of the ${ }^{15} \mathrm{~N}-\mathrm{HSQC}$ chemical shifts after addition of CBP to ${ }^{15} \mathrm{~N}$ labeled HIF-1a. Using these same techniques the $\mathrm{aC}$ domain (233-425) of fibrin was expressed in minimal media to produce ${ }^{15} \mathrm{~N}$ - 
labeled protein. ${ }^{15} \mathrm{~N}-\mathrm{HSQC}$ was then utilized to monitor peak dispersion (structure formation) when FXIII-A $A_{2}$ was added to the solution. 


\section{Materials and Methods}

Preparation of $\alpha C$ domain (233-425) DNA stocks

The aC-domain (233-425) pGEX-6P-1 plasmid (GE Healthcare, Piscataway, NJ) DNA was a generous gift from Helen Philippou, Robert Ariens and Kerrie Smith at the University of Leeds, Leeds Institute of Genetics Health and Therapeutics (121). It should be noted that all materials used during transformation, expression and purification were sterile. DH5a E. coli cells (Invitrogen, Carlsbad, CA) were transformed to build an aC (233-425) DNA stocks. The first step in transforming the DH5a cells was transferring $\sim 5 \mathrm{ng} \mathrm{aC}$ (233-425) DNA into a $50 \mu$ laliquot of DH5a competent cells in a $1.5 \mathrm{ml}$ tube.

This DNA/E. coli mix was placed on ice and incubated for $30 \mathrm{~min}$. Following 30 min on ice the cells were heat shocked for 20 seconds in a $42{ }^{\circ} \mathrm{C}$ water bath and then returned to the ice for an additional $2 \mathrm{~min}$. The transformation was then brought to temperature with the addition of $950 \mu 137^{\circ} \mathrm{C}$ LB media (Appendix C). The cell mix was then incubated for $1 \mathrm{hr}$ at $37^{\circ} \mathrm{C}$ while shaking at $225 \mathrm{rpm}$. After incubation, if transformation efficiency is low, the cells could be concentrated by spinning for $2 \mathrm{sec}$ at $10,000 \mathrm{rpm}$ and removing $500 \mu \mathrm{l}$ supernatant prior to resuspending cells. In most cases this step was unnecessary and $75 \mu \mathrm{l}$ of the transformed cells were plated on LB agar + Ampicillin plates (Appendix C). The plates incubated for $16 \mathrm{hr}$ at $37^{\circ} \mathrm{C}$. Following transformation, a single intact colony was utilized to inoculate $4 \mathrm{ml} \mathrm{LB}$ agar containing $100 \mu \mathrm{g} / \mathrm{ml}$ Ampicillin. The $4 \mathrm{ml}$ culture was incubated at $37{ }^{\circ} \mathrm{C}$ with shaking at $225 \mathrm{rpm}$ for $16 \mathrm{hr}$. The 
DNA was then purified using the QIAprep Miniprep kit (Qiagen Inc., Valencia , CA). Following purification, the DNA concentration was determined using a Nanodrop ND-1000 Spectrophotometer (Thermo Scientific, Wilmington, DE). A typical DNA yield for an aC-DNA Miniprep was $60-80 \mathrm{ng} / \mathrm{ml}$. Purified DNA was also sent for sequencing using pGEX-6P-1 $3^{\prime}$ and 5 ' primers (GE Healthcare, Piscataway, NJ).

Transformation of $\alpha C$ domain (233-425) in BL21 Gold (DE3) cells

Once DNA stocks were established, BL21 Gold (DE3) cells were utilized to express the aC-domain (233-425) for purification. A $1-2 \mu$ l aliquot totaling 10 - $25 \mathrm{ng}$ DNA was added to a $14 \mathrm{ml}$ round bottom tube with $100 \mu \mathrm{l}$ BL21-Gold (DE3) E. coli cells (Agilent Technologies, La Jolla, Ca). The BL21 cells were incubated on ice for $30 \mathrm{~min}$ then heat shocked in a water bath for $20 \mathrm{sec}$ at $42^{\circ} \mathrm{C}$. Following the $20 \mathrm{sec}$ heat shock, the cells were placed on ice for additional 2 $\min$. The cells were then brought to temperature through the addition of $1 \mathrm{ml}$ prewarmed $\left(42^{\circ} \mathrm{C}\right) \mathrm{SOC}$ media (Appendix C). After addition of SOC media, the cells $(150 \mu \mathrm{l})$ were then plated on LB agar + Ampicillin and incubated overnight at $37^{\circ} \mathrm{C}$.

Following incubation, a colony of cells was transferred to $5 \mathrm{ml} \mathrm{LB}$ broth containing $100 \mu \mathrm{g} / \mathrm{ml}$ Ampicillin. The liquid culture was placed in the incubator where the cells were incubated at $37^{\circ} \mathrm{C}$ for $16-20 \mathrm{hr}$ and then utilized to establish BL21-Gold cell stocks. Stocks were made by adding $5 \mathrm{ml} 50 \%$ glycerol to the cells then aliquoting and storing them at $-70^{\circ} \mathrm{C}$ for later use. 
Expressing aC (233-425) in BL21 Gold (DE3) cells

The expression of $\alpha C$ (233-425) in transformed BL21 stocks can take place in any number of volumes. For example sake the steps used for a $2 \mathrm{~L}$ culture are described. First a $200 \mathrm{ml}$ starter (or seed) culture was grown overnight after inoculation with the frozen BL21 stocks. The starter culture consisted of $180 \mathrm{ml}$ LB broth, $20 \mathrm{ml}$ phosphate buffer and $200 \mu \mathrm{l} 100 \mathrm{mg} / \mathrm{ml}$ Ampicillin (Appendix C). The following day prior to addition of cells, $1800 \mathrm{ml}$ Terrific Broth, $200 \mathrm{ml}$ phosphate buffer and $2 \mathrm{ml} 100 \mathrm{mg} / \mathrm{ml}$ Ampicillin was prepared in a $4 \mathrm{~L}$ flask. An aliquot was taken and analyzed on the Cary 50 UVNIS to establish a baseline $\left(A_{600}=0\right)$. Once a baseline was established for $T$ $=0$, the $200 \mathrm{ml}$ starter culture was added to the flask.

The culture $(2 \mathrm{~L})$ was shaken $(225 \mathrm{rpm})$ at $37^{\circ} \mathrm{C}$ until the absorbance (cell growth) $A_{600}=0.8-1.0$. At which time $2.2 \mathrm{ml}$ of $1 \mathrm{M}$ Isopropyl $\beta-\mathrm{D}-1-$ thiogalactopyranoside (IPTG), a molecular mimic of a lactose metabolite, was added to the culture. The addition of IPTG triggers lac operon transcription on the pGEX-6P-1plasmid leading to the over expression of aC (233-425). After addition of IPTG, the culture was shaken $(180 \mathrm{rpm})$ at $30^{\circ} \mathrm{C}$ for 16 hours. The cells were then transferred to centrifuge bottles and harvested during sequential 10 min spins at $5000 \mathrm{~g}$ and $4{ }^{\circ} \mathrm{C}$. The harvested cell pellets were resuspended in $180 \mathrm{ml}$ ice cold E. coli wash buffer (Appendix C) by stirring ( $800 \mathrm{rpm}$ ) for $\sim 40$ min. Lastly, the cell suspension was transferred to $50 \mathrm{ml}$ conical tubes and centrifuged in a swinging bucket rotor $45 \mathrm{~min}$ at $5000 \mathrm{~g}$ and $4{ }^{\circ} \mathrm{C}$. The 
supernatant was discarded and the cell pellets were placed at $-20^{\circ} \mathrm{C}$ until purification.

Expressing ${ }^{15} \mathrm{~N}-\alpha C$ (233-425) in BL21 Gold (DE3) cells with Minimal Media

For isotopically labeling aC (233-425) the transformed BL21 cells were grown in minimal media. The recipes used for M9 salts and M9 media were adapted from Marley et al. and Paliy, et al (114-115). The seed culture for minimal media expression was grown in standard LB broth. For a $2 \mathrm{~L}$ culture, two sterile $50 \mathrm{ml}$ conical tubes containing $36 \mathrm{ml}$ LB broth were inoculated with the frozen stocks of transformed BL21 cells. The starter culture was grown overnight at $37^{\circ} \mathrm{C}$ while shaking at $225 \mathrm{rpm}$. The following morning the two starter cultures were spun at $5000 \mathrm{~g}$ for $10 \mathrm{~min}$ and the LB broth was discarded. Being careful not to disturb the peliet, the pellet was

Table 5: Components of 1 L. 5× M9 salts and $1 \times$ concentration. The mass needed for $1 \mathrm{~L} 5 \times \mathrm{M} 9$ salts and the final salt concentration after dilution to $1 x$.

washed $2 \times$ with $5 \mathrm{ml}$

1X M9 salts (Table

5). The pellet was

\begin{tabular}{lcc}
\hline & Mass $(\mathrm{g})$ & $1 \times(\mathrm{mM})$ \\
\hline $\mathrm{KH}_{2} \mathrm{PO}_{4}$ & 30 & 44 \\
$\mathrm{Na}_{2} \mathrm{HPO}_{4}$ & 108 & 96 \\
$\mathrm{NaCl}$ & 2.5 & 9 \\
\hline
\end{tabular}

then resuspended in $2 \mathrm{ml} 1 \times \mathrm{M} 9$ salts and transferred to $2 \mathrm{~L}$ of $\mathrm{M} 9$ media (Table 5 and Table 6). Prior to addition of cells to the M9 media, an aliquot was taken and used to establish an $A_{600}$ baseline. Cell growth was monitored and the cells were induced with the addition of $2 \mathrm{ml} 1 \mathrm{M} \mathrm{IPTG}$. The cells were then incubated overnight at $30^{\circ} \mathrm{C}$ while shaking at $180 \mathrm{rpm}$. The $2 \mathrm{~L}$ culture was then spun down, washed and spun down again before freezing in the same manner previously described for growth in LB broth. 
Table 6: Recipe for M9 minimal media. This table outlines all the components added to 1 $\mathrm{L}$ of $\mathrm{M} 9$ growth media. For each component, the volume added as well as the final concentration are given.

\begin{tabular}{ccc}
\hline Volume $(\mathrm{ml})$ & M9 Media component & Final Concentration \\
\hline 200 & $5 \times \mathrm{M}$ salts & $1 \times$ \\
1.00 & $1 \mathrm{M} \mathrm{MgSO}_{4}$ & $1.0 \mathrm{mM}$ \\
1.00 & $200 \mathrm{mM} \mathrm{CaCl}_{2}$ & $0.2 \mathrm{mM}$ \\
4.00 & ${ }^{a} 0.25 \mathrm{~g} / \mathrm{ml} \mathrm{NH}_{4} \mathrm{Cl}$ & $1.0 \mathrm{~g} / \mathrm{l}$ \\
0.05 & $20 \% \mathrm{Thiamine} \mathrm{HCl}$ & $0.001 \%$ \\
1.00 & $100 \mathrm{mg} / \mathrm{ml} \mathrm{Ampicillin}$ & $0.1 \mathrm{mg} / \mathrm{ml}$ \\
40.0 & $20 \%$ Dextrose & $0.8 \%$ \\
753 & Sterile dH ${ }_{2} \mathrm{O}$ & \\
\hline ressing isotopically labeled proteins, ${ }^{15} \mathrm{NH} 4 \mathrm{Cl}$ was utilized as opposed to $\mathrm{NH}_{4} \mathrm{Cl}$.
\end{tabular}

Purification of $\alpha C$ (233-425)

The $\alpha \mathrm{C}(233-425)$ produced during growth in LB media or M9 media was purified using the same process. Hot water was run over tubes and a spatula utilized to help transfer the frozen pellets to a clean $500 \mathrm{ml}$ beaker. The tubes were rinsed with and resuspended in ice cold PBS (4 ml PBS/100 ml culture - 88 $\mathrm{ml}$ PBS for 2L). The cells were stirred gently at room temperature to resuspend. Once resuspended, $888 \mu 100 \mathrm{mg} / \mathrm{ml}$ Lysozyme [1 $\mathrm{mg} / \mathrm{ml}$ ] (the [ ] indicate assay concentration) and $88.8 \mu \mathrm{l} 1 \mathrm{M} \mathrm{DTT}[1 \mathrm{mM}]$ was added to the cell suspension and incubated at room temperature, stirring gently, for $30 \mathrm{~min}$. The protease inhibitors listed in Table 7 were added to the cell suspension and incubated for $30 \mathrm{~min}$ at $4{ }^{\circ} \mathrm{C}$ stirring gently. Following incubation with protease inhibitors, $222 \mu \mathrm{l}$ $100 \mathrm{mM}$ phenylmethylsulfonyl fluoride $[0.5 \mathrm{mM}]$ and $449 \mu \mathrm{l} 10 \%$ Sodium Deoxycholate were added and the cell mix was stirred for 30 more minutes. The addition of $20 \%$ Triton X100 [1\%] was done forcefully then $47.3 \mu 110 \mathrm{mg} / \mathrm{ml}$ DNase $1[5 \mu \mathrm{g} / \mathrm{ml}]$ was introduced with $472.5 \mu \mathrm{l} 1 \mathrm{M} \mathrm{MgCl}_{2}[5 \mathrm{mM}]$. This was 
Table 7: The protease inhibitors utilized in during purification of recombinant protein.

\begin{tabular}{ccc}
\hline $\begin{array}{c}\text { Added to 2 } \\
\text { Culture }\end{array}$ & Protease Inhibitor & Assay Concentration \\
\hline $88.9 \mu \mathrm{l}$ & $2 \mathrm{mg} / \mathrm{ml}$ Aprotinin & {$[2 \mu \mathrm{g} / \mathrm{ml}]$} \\
$88.9 \mu \mathrm{l}$ & $1 \mathrm{mM}$ Pepstatin A & {$[1 \mu \mathrm{M}]$} \\
$222 \mu \mathrm{l}$ & $4 \mathrm{M}$ Leupeptin & {$[10 \mu \mathrm{M}]$} \\
$0.069 \mathrm{~g}$ & Benzamidine & {$[4 \mathrm{mM}]$} \\
\hline
\end{tabular}

incubated for $10 \mathrm{~min}$ at $4^{\circ} \mathrm{C}$ with stirring then quenched with the addition of 1.15 $\mathrm{ml} 0.5 \mathrm{M}$ EDTA [ $6 \mathrm{mM}]$. The cell suspension was then transferred to centrifuge tubes and spun for 20 minutes at $22,000 \mathrm{~g}$. The supernatant was filtered using $0.22 \mu \mathrm{m}$ Supor machV $150 \mathrm{ml}$ filter-flasks (Nalgene, Rochester, NY).

The purification process takes advantage of GST that is co-expressed with the $\alpha \mathrm{C}(233-425)$. Utilizing an AKTA prime system, with GST specific GSTrap $^{\text {TM }}$ HP Columns (GE Healthcare, Piscataway, NJ) the GST-aC was trapped on the column (Figure 37). After overnight digestion with PreScission Protease, 1 unit per $100 \mu \mathrm{g}$ protein, (GE Healthcare, Piscataway, $\mathrm{NJ}$ ) the free $\alpha \mathrm{C}$ $(233-425)$ was eluted from the column. To ensure a clean sample for future NMR studies the unlabeled $\alpha C(233-425)$ product was cleaned using a Detergent Removal Spin Column (Pierce, Rockford, IL). The ${ }^{15} \mathrm{~N}$ aC $(233-425)$ was dialyzed into $1 \times$ PBS and all recombinant $\alpha C$ was concentrated using an Amicon Ultra centrifugal concentration devices MWCO $=10,000$ (Millipore, Billerica, MA). The concentration was established using an extinction coefficient of $41,480 \mathrm{M}^{-1} \mathrm{~cm}^{-1}$ calculated using ExPASy ProtParam (http://web.expasy.org/protparam/). 
A

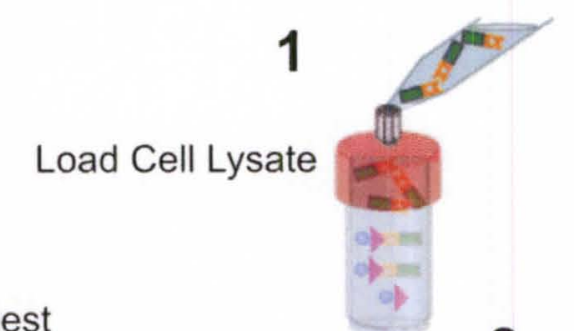

On-Column Digest

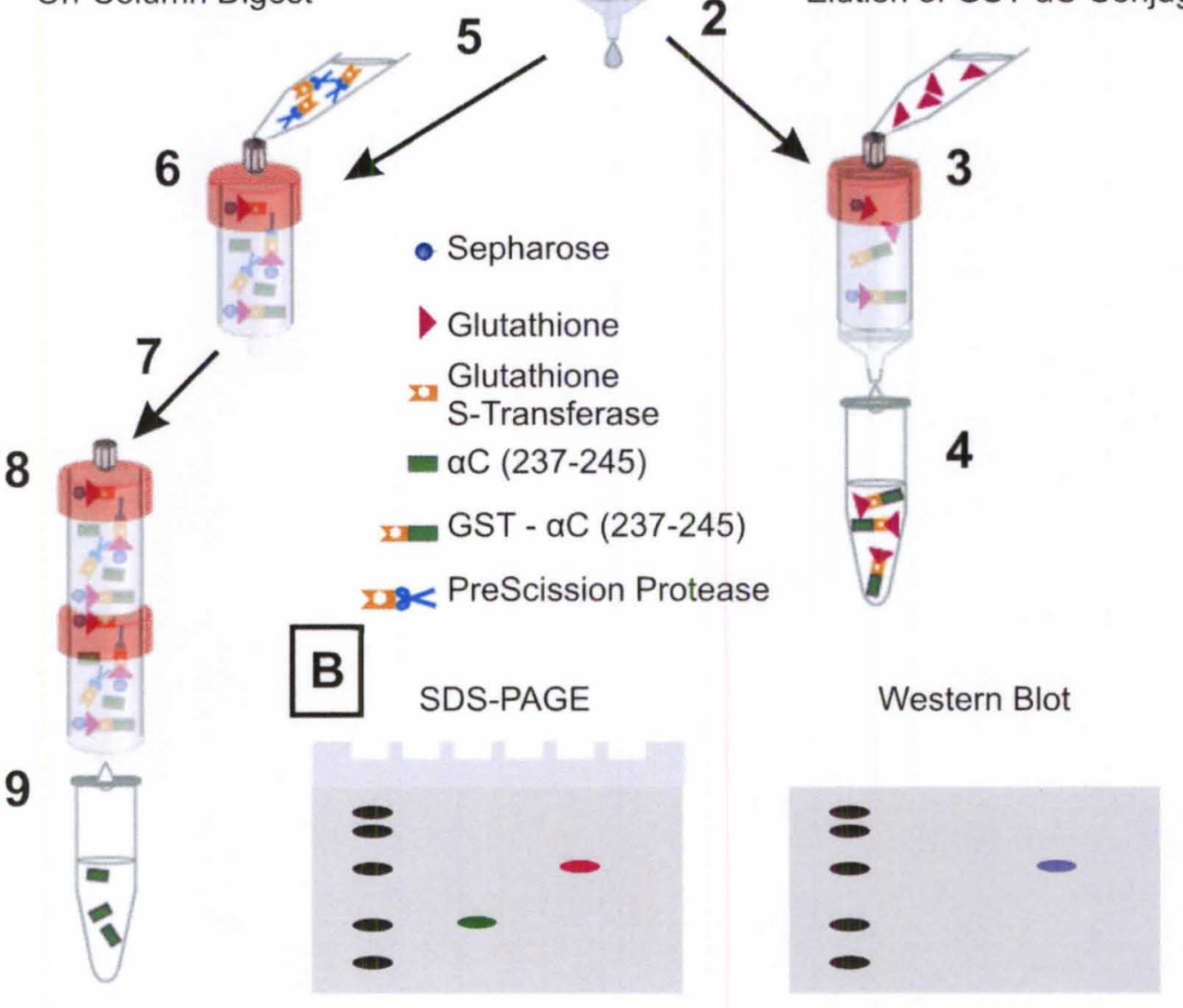

Figure 37: Visualization of $\alpha \mathrm{C}$ (233-425) purification following expression in BL21 Gold E. coli cells.

A) There are two different schemes shown; one for eluting aC (233-425) still bound to the GST linker (steps $2-4)$, and the other describes an on column digest to elute just $\alpha \mathrm{C}$ (233-425) from the GSTrap column (steps 5 -9). Step (1) is the addition of cell lysate to the GSTrap (GST affinity column). For elution of the GST- $a$ C conjugate, the column was rinsed (2), and reduced glutathione is pumped across the column (3) releasing the GST- $\alpha \mathrm{C}$ from the glutathionesepharose column material. The GST- $\alpha \mathrm{C}(233-425)$ fraction is collected and stored at $4{ }^{\circ} \mathrm{C}$ until further analysis (4). For on-column digests, after the addition of cell lysate the column was rinsed (5). PreScission protease was then added to the column (6) and allowed to incubate at $4{ }^{\circ} \mathrm{C}$ overnight (7). The following morning an additional GSTrap column was added to capture any possible GST that may be released (8) and the recombinant $\alpha \mathrm{C}$ (233-425) was eluted and stored at $4{ }^{\circ} \mathrm{C}$ until further analysis (9).

B) Representative SDS-PAGE gel and Western Blot image. In the SDS-PAGE gel the green band represents $\alpha C$ (233-425) and the pink band GST- $\alpha \mathrm{C}$ (233-425). The GST specific Western Blot is representative of a blot containing un-cleaved GST- aC (233-425). 
SDS-PAGE and Western Blot

All expression products were confirmed using SDS-PAGE. The $15 \%$ resolving gel was prepared by combining $2.4 \mathrm{ml} \mathrm{dH} 2 \mathrm{O}, 2.5 \mathrm{ml} 1.5 \mathrm{M}$ Tris $(\mathrm{pH}=8.8), 5.0 \mathrm{ml}$ $30 \%$ acrylamide and $0.1 \mathrm{ml} 10 \%$ SDS. The acrylamide polymerization was initiated with the addition of $50 \mu \mathrm{l}$ Ammonium Persulfate (APS) and $5.0 \mu \mathrm{l}$ Tetramethylethylenediamine (TEMED). The $4.0 \%$ stacking gel which was poured on top of the resolving gel consisted of: $6.1 \mathrm{ml} \mathrm{dH} 2 \mathrm{O}, 2.5 \mathrm{ml} 0.5 \mathrm{M}$ Tris $(\mathrm{pH}=6.8), 1.3 \mathrm{ml} 30 \%$ acrylamide and $0.1 \mathrm{ml} 10 \% \mathrm{SDS}$. Once again, the polymerization was initiated with $50 \mu \mathrm{I}$ APS and $10 \mu \mathrm{l}$ TEMED. Samples were mixed with non-reducing sample buffer. The Bio-Rad MiniGels (Hercules, CA) were run at $200 \mathrm{~V}$ for $1 \mathrm{hr}$. The PAGE gel was then either subjected to Western Blot or Coomassie Blue $(0.1 \%$ Coomassie Blue, $40 \% \mathrm{MeOH}, 10 \%$ Acetic Acid and $50 \% \mathrm{dH}_{2} \mathrm{O}$ ) staining. For staining, gels were placed in Coomassie Blue stain overnight then destained for approximately 3 hours in Destain $(40 \% \mathrm{MeOH}$, $10 \%$ Acetic Acid and $50 \% \mathrm{dH}_{2} \mathrm{O}$ ). Gels were then imaged using a Gel Doc ${ }^{\mathrm{TM}}$ XR+ System (Bio-Rad, Hercules, CA).

After SDS-PAGE, western blots were utilized to confirm the presence of GST - aC (233 - 245) or determine whether all GST was removed after oncolumn PreScission protease digestion. The gel, fiber pad, filter paper, and nitrocellulose membrane (NitroBind, Osmonics Inc.) were equilibrated by soaking them in the Western Blot Transfer Buffer (25 mM Tris, $192 \mathrm{mM}$ Glycine and $20 \%$

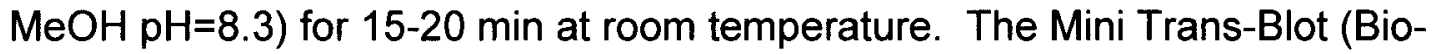


Rad Hercules, CA) was loaded as per instructions and run at $100 \mathrm{~V}$ for $1 \mathrm{Hr}$ at 4 ${ }^{\circ} \mathrm{C}$. To decrease non-specific binding, the nitrocellulose membrane was placed in a $3 \%$ BSA Blocking Solution in PBST with stirring overnight at room temperature. The following morning, the membrane was washed in PBST then placed in a solution containing 1:5000 $\alpha$-GST-HRP (horseradish peroxidase) (GE Healthcare, Piscataway, $\mathrm{NJ}$ ). After a series of 5 rinses in $1 \times$ PBST, the membrane was developed using 1-Step TMB-Blotting solution, a blue TMB $\left(3,3^{\prime}, 5,5^{\prime}\right.$-tetramethylbenzidine) peroxidase which is an HRP substrate (Pierce, Rockford, IL). Membranes were then dried and visualized on the Gel Doc ${ }^{\mathrm{TM}} \mathrm{XR}+$ System (Bio-Rad, Hercules, CA).

${ }^{15} N^{-1} H H S Q C$

After the ${ }^{15} \mathrm{~N}$ labeled and unlabeled $\mathrm{aC}$ domain $(233-425)$ were dialyzed and concentrated, they were loaded into NMR Shigemi tubes. The concentration in the NMR ranged from $<100 \mu \mathrm{M}$ for the unlabeled $\alpha \mathrm{C}$ domain $(233-425)$ to $>$ $200 \mu \mathrm{M}$ for the ${ }^{15} \mathrm{~N}$ labeled material and all samples were run in the presence of $10 \% \mathrm{D}_{2} \mathrm{O}$. The $\mathrm{pH}$ of NMR samples was not adjusted below 6.6 due to a theoretical pl of 6.28 (http://web.expasy.org/protparam/). Two NMR experiments were run to establish the feasibility of determining $a C$ domain $(233-425)$ structure. $\alpha \mathrm{C}$ domain $(233-425)$ was first analyzed with a Watergate ${ }^{1} \mathrm{H}$ TOSCY and then the ${ }^{15} \mathrm{~N}-\alpha \mathrm{C}$ domain $(233-425)$ was utilized in ${ }^{15} \mathrm{~N}-{ }^{1} \mathrm{H}$ HSQC. To determine if the $\mathrm{aC}$ domain $(233-425)$ adopts a defined compact structure in the presence of $\mathrm{FXIII- \textrm {A } _ { 2 }}$ a ${ }^{15} \mathrm{~N}-{ }^{1} \mathrm{H}$ HSQC was performed. For this analysis, 350 $\mu \mathrm{l}$ of $210 \mu \mathrm{M}{ }^{15} \mathrm{~N}$ aC $(233-425)$ was used to solubilize $3.2 \mathrm{mg}$ dialyzed and lyophilized FXIII-A . The final concentrations in the NMR tube were $110 \mu \mathrm{M}$ 
FXIII- $A_{2}$ and $210 \mu \mathrm{M}{ }^{15} \mathrm{~N}$ aC $(233-425)$. All NMR experiments were performed on a 16.4 Tesla 700 Varian Inova MHz NMR or an 18.8 Tesla $800 \mathrm{MHz}$ Varian Inova NMR.

The NMR data shown in Chapter V were collected on the Varian Inova $800 \mathrm{MHz}$ spectrometer which is equipped with a triple resonance probe and pulsed-field triple axis gradients. ${ }^{1} \mathrm{H}-{ }^{15} \mathrm{~N}$ HSQC experiments were run on the ${ }^{15} \mathrm{~N} \alpha C$ (233-425) in the absence and presence of unlabeled FXIII $A_{2}$. For both systems, the ${ }^{1} \mathrm{H}$ frequency was $799.708 \mathrm{~Hz}$ and the sweep width was set at 12000 whereas the ${ }^{15} \mathrm{~N}$ frequency was $81.043 \mathrm{~Hz}$ and the sweep width was set at 2300. Spectra were collected with 1800 complex points in the ${ }^{1} \mathrm{H}$ dimension and 100 increments in the ${ }^{15} \mathrm{~N}$ dimension. The number of transients was 8 . All HSQC experiments were run at $20^{\circ} \mathrm{C}$ and water was used as a reference. The NMR data were processed using NMRPipe and NMRDraw version 6.5 (123) and the results visualized using Sparky version 3.1(124). The ppm reference for the center of both 2D spectra was $4.821 \mathrm{ppm}$ and $119.990 \mathrm{ppm}$. Sparky was also used to overlay the HSQC spectrum of ${ }^{15} \mathrm{~N}$ aC $(233-425)$ on to the spectrum of the protein-protein complex involving ${ }^{15} \mathrm{~N} \alpha \mathrm{C}(233-425)$ and FXIII $A_{2}$. The Overlay Views dialog box was used. Both spectra had been subjected to the same experimental parameters, referencing, and data processing strategies. 


\section{Results}

$\alpha C(233-425)$ Expression in LB Broth

To establish an expression system in the lab two systems were chosen for method development, FXIII- $A_{2}$ and aC (233 - 425). The aC (233-425) gene was cloned into pGEX-6P-1 vectors (Figure 38). The location of the $\alpha \mathrm{C}$ gene insert was ideal for PreScission protesase cleavage and GST cleavage during purification.

The first objective was to transform the cloned pGEX-6P-1 vector into

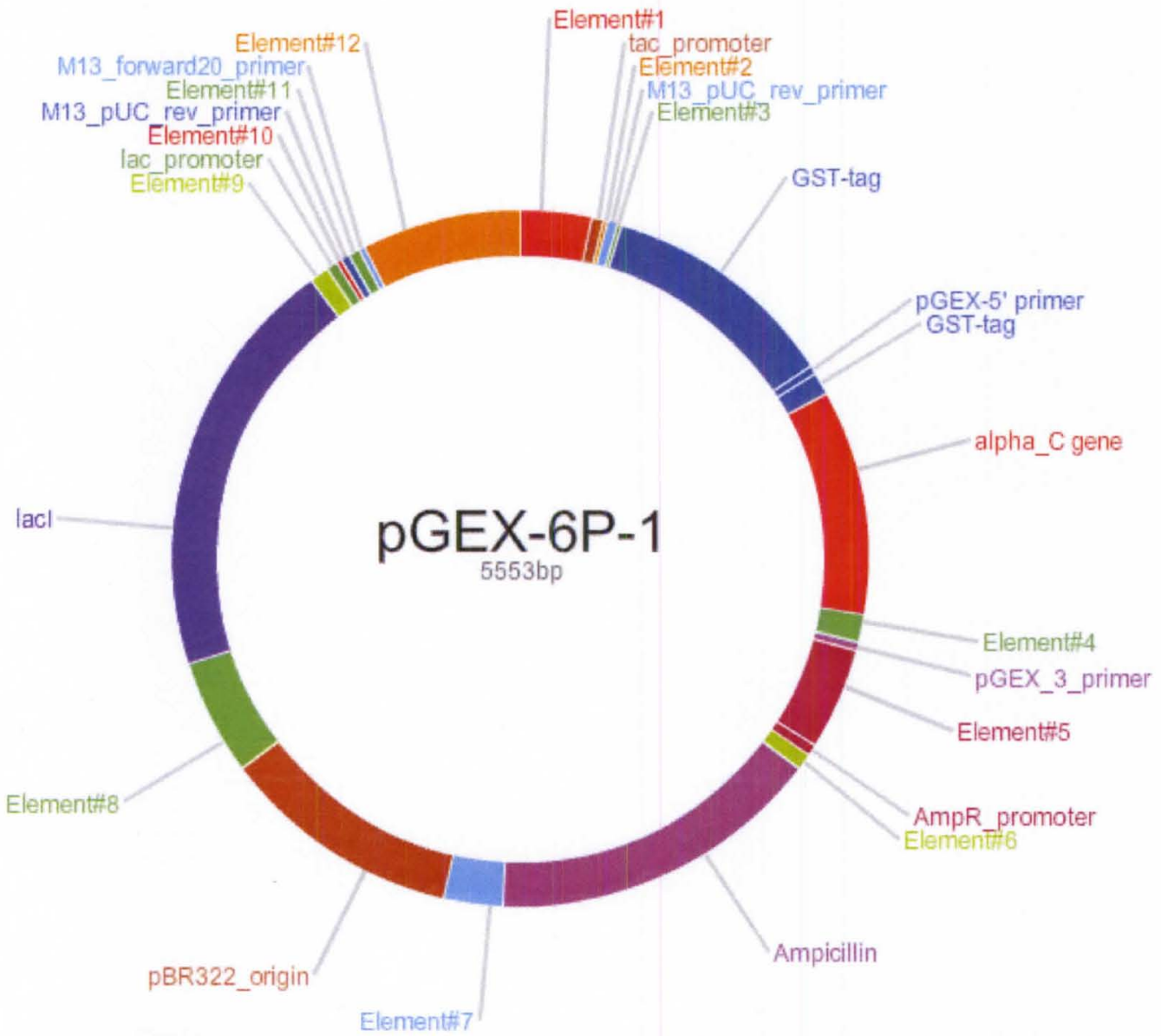

Figure 38: Map of pGEX-6P-1 vector. Elements of interest are labeled. The elements to note are the tac_promotor (Orange), GST-tag which contains the pGEX-5' primer (Blue), alpha_C gene (Red). pGEX_3_primer and the Ampicillin gene (Purple). Image created using DNA 2.0 Gene Designer 2.0 software. 
DH5 $\alpha$ cells in order to build a series of DNA stocks. Using the transformation

methods set forth by Smith et. al., the DNA yields from a standard MiniPrep were consistently $60-80 \mathrm{ng} / \mathrm{ml}(121)$. This allowed for the storage of several DNA aliquots to be used in future studies. To confirm that the purified DNA contained the aC $(233-425)$ gene, it was sequenced using the pGEX-6P-1 $3^{\prime}$ and $5^{\prime}$

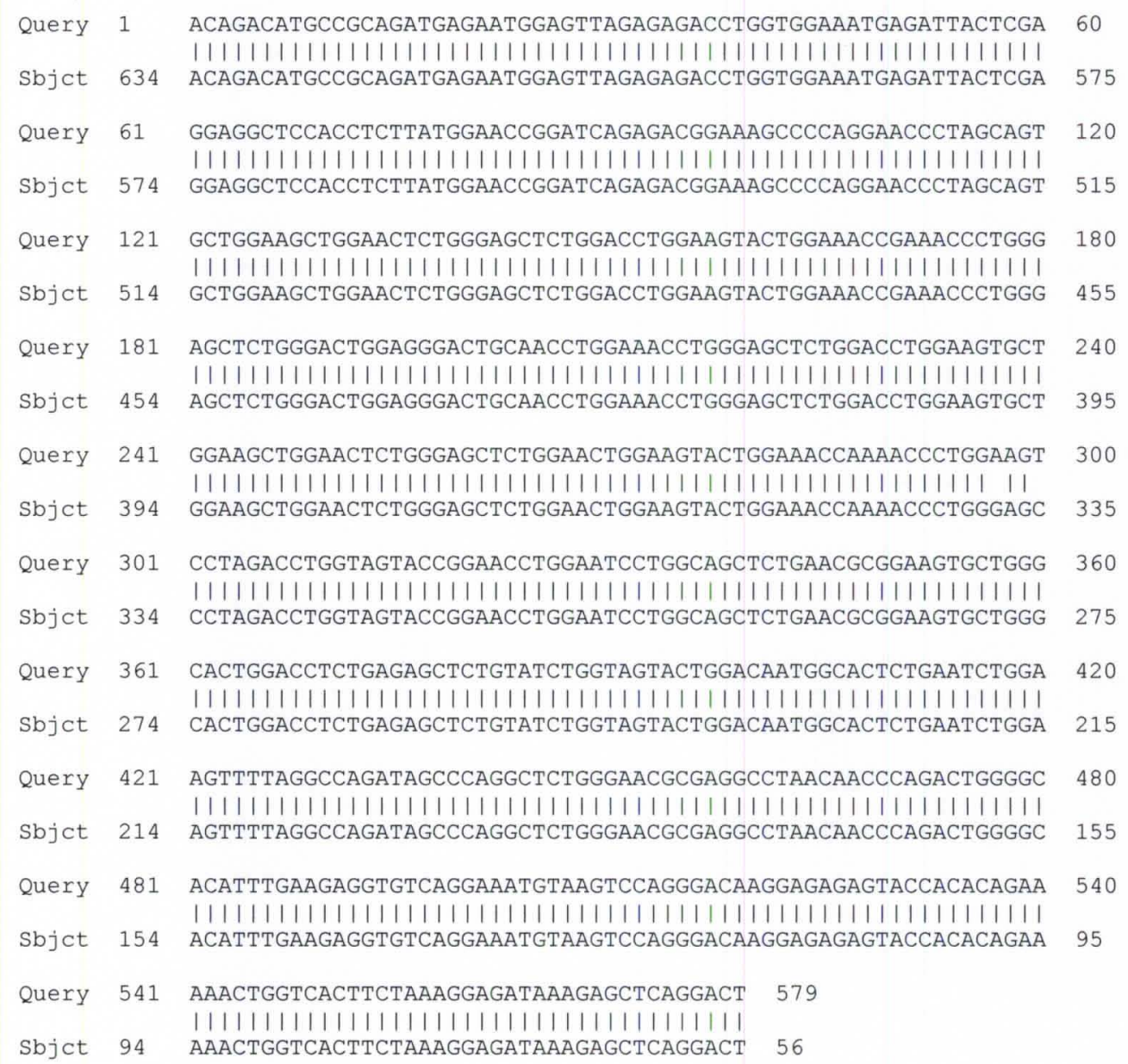

Figure 39: Alignment of 3' primer sequence data (top) and aC (233 - 425) gene sequence (bottom). primers (3' CCGGGAGCTGCATGTGTCAGAGG and 5' GGGCTGGCAAGCCACGTTTGGTG) (Figure 38). Both the 5' and 3' primers produced sequence data that confirmed the entire $\alpha \mathrm{C}(233-245)$ construct sequence (Figure 39). The two mis-hits (nucleotide 297 and 300) in Figure 39 
were both mutations that did not lead to a change in amino acid. The $\mathrm{G}$ to $\mathrm{A}$ (GG(A)) mutation at nucleotide 297 remains a glycine and the $C$ to $T(A G(T))$ mutation at nucleotide 300 remains a serine.

Initially, protein expression took place in standard LB media. The DNA collected from DH5 $\alpha$ cells was successfully transformed into BL21 Gold (DE3) cells. During the first attempts at collecting purified protein from a $2 \mathrm{~L}$ culture, the decision was made to elute the uncleaved GST- $\alpha \mathrm{C}(233$ - 425) from the GSTrap column. The next step was developing a method to release the GST from the $a \mathrm{C}$ $(233-425)$. This was done via an on-column digest (Figure 37) which utilized PreScission protease. PreScission protease selectively cleaves between Gln and Gly in a sequence (Leu Glu Val Leu Phe Gln | Gly Pro) found N-terminally to aC (233 - 425). Therefore, after

transformation and purification the cell

extract was loaded on the GSTrap column and then the column was removed from the AKTAprime system. At which time

PreScission protease was added and incubated on-column overnight at $4{ }^{\circ} \mathrm{C}$ to allow for cleavage between aC (233 - 425) $M W=19582 \mathrm{~g} / \mathrm{mol}$ and GST $\mathrm{MW}=27,000$ $\mathrm{g} / \mathrm{mol}$. The GST and GST-tagged PreScission protesase remain on the columan and the cleaved $\alpha \mathrm{C}(233-425)$

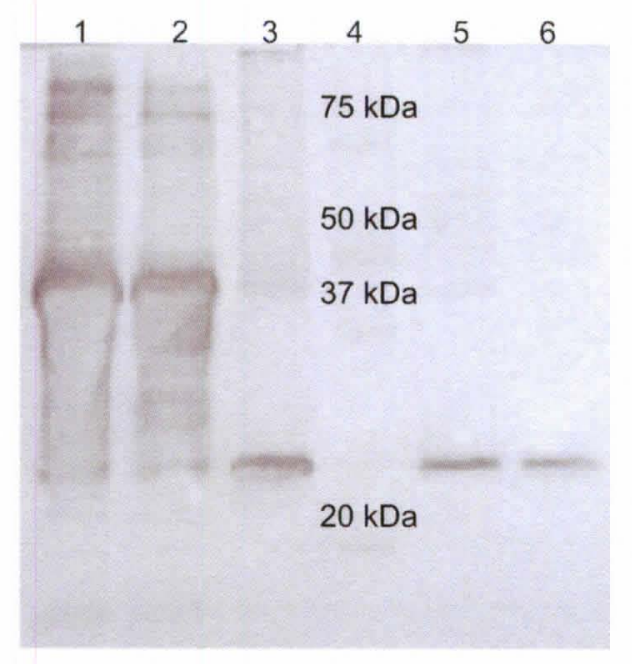

Figure 40: Representative GST specific Western Blot. Lane (1) was collected from the waste line while loading the column with cell extract. (2) was the cell extract. (3) was the concentrated $\alpha \mathrm{C}$ product with GST contaminant. (4) was the ladder. (5 and 6) were collected during GST elution. 
was eluted the following morning. SDS-PAGE and a GST-specific western blot were run to evaluate the expression and purification process (Figure 40). The GST-specific western blot exposed that GST was eluting with the aC (233 - 425); therefore, in future protein elutions an additional GSTrap column was added post on-column digestion to sequester any GST that may be co-eluting with $\mathrm{aC}(233$ 425).

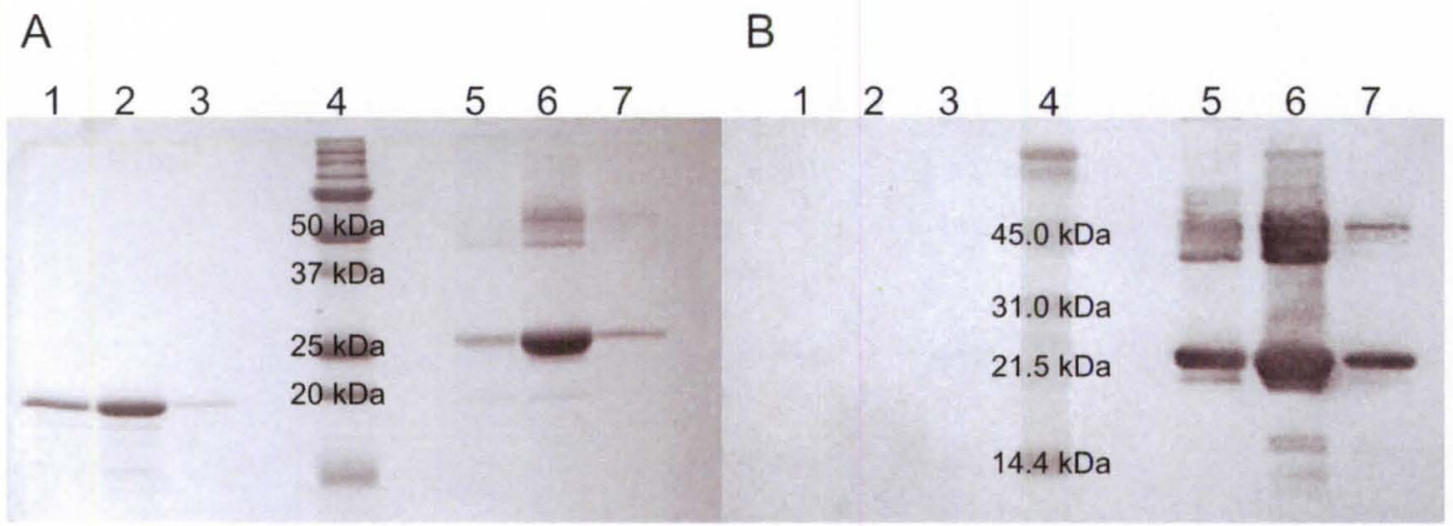

Figure 41: Representative SDS-PAGE and western blot confirming proper aC (233 - 425) purification and GST cleavage. (A) The first three wells in the SDS-PAGE illustrate the $\alpha \mathrm{C}(233-425)$ was sufficiently cleaved and eluted. The GST elution illustrated in wells $5-7$ demonstrated minimal residual $\mathrm{aC}(233-425)$. This carryover was not affected by increasing the column wash-time/volume between $\mathrm{aC}(233-425)$ and GST elution. (B) Wells $1-3$ of the GST-specific western blot demonstrated a clean aC $(233-425)$ elution free from GST. Wells $5-7$ contain the cleaved GST fraction.

\section{Expresssion in minimal media}

Expression in minimal media proved to be just as successful as $\alpha \mathrm{C}(233-$ 425) expression in under standard nutrient conditions. The true yields of both LB and minimal media expressions are difficult to report due to loss of material during storage, dialysis, and surfactant removal but both methods were successful in producing enough protein for NMR analysis. During minimal media expression and purification, the GST that had eluted with purified $\alpha \mathrm{C}(233-425)$ previously was eliminated (Figure 41B). One issue that did occur in all 
expressions was mild carryover after $\alpha C(233-425)$ elution. The $\alpha C(233-425)$ that eluted with the GST was seen as a faint band around $20 \mathrm{kDa}$ in the GST fractions (Figure 41A). This band occurred even after increasing the column wash time following aC $(233-425)$ elution. Another possible issue is the band in the western blot around $45 \mathrm{kDa}$. This could possibly be conjugated GST-aC (mw $=46,582 \mathrm{kDa}$ ). If that were the case, additional PreScission protease should yield more aC $(233-425)$ and decrease the intensity of the band at $45 \mathrm{kDa}$. This was attempted and the $45 \mathrm{kDa}$ band was still quite prominent; therefore, the large band around $45 \mathrm{kDa}$ may be due to non-specific binding. The $\mathrm{aC}(233-$ 425) expression system proved to be quite versatile and allowed for protein production under standard nutrient conditions and under minimal media with ${ }^{15} \mathrm{~N}$ $\mathrm{NH}_{4} \mathrm{Cl}$.

$\alpha C(233-425){ }^{15} \mathrm{~N}-H S Q C$

Although the ultimate goal of expressing aC $(233-425)$ in ${ }^{15} \mathrm{~N}$-labeled minimal media was to enable NMR observations, the first NMR experiments utilized unlabeled $\alpha C(233-425)$. A valuable lesson was learned during our first TOSCY analysis of unlabeled aC $(233-425)$. There was substantial contamination from a small molecule in our sample. After reviewing the purification procedures, the problem was identified as Triton $X-100$ which is added to help solubilize the protein before centrifugation. The Triton X-100 concentration is $1 \%$ and the critical micelle concentration is $0.1 \%$. The Triton $\mathrm{X}$ 100 micelles contain 628 Triton units/micelle therefore has an apparent molecular weight of $\sim 90 \mathrm{kDa}$. This apparent molecular weight caused the Triton 


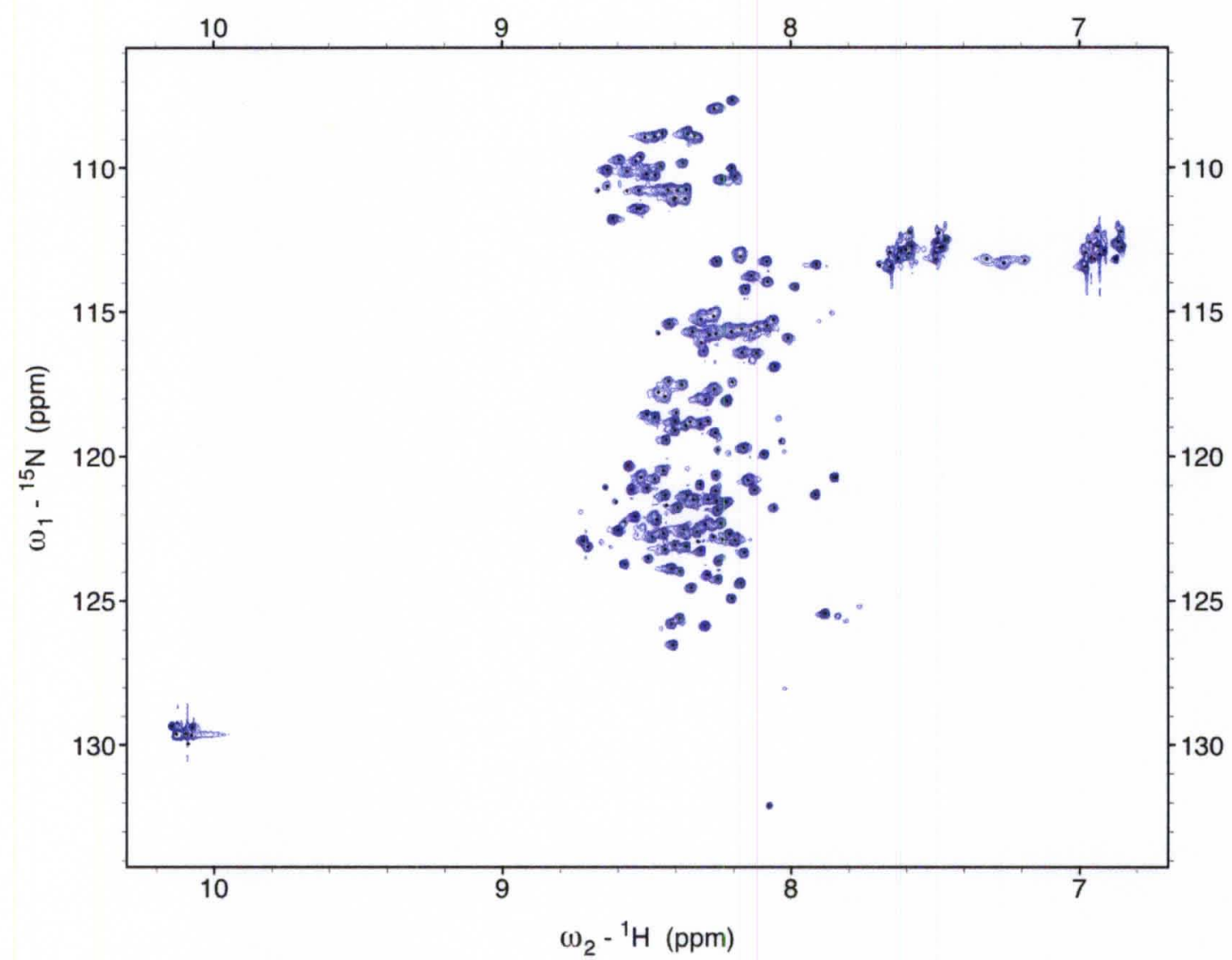

Figure 42: ${ }^{15} \mathrm{~N}-\mathrm{HSQC}$ spectra of ${ }^{15} \mathrm{~N}-\mathrm{aC}(233-425)$ conducted on an $800 \mathrm{MHz}(18 \mathrm{~T})$ Varian Inova NMR at $20^{\circ} \mathrm{C}$. The assignments can be viewd in appendix $\mathrm{F}$.

$\mathrm{X}-100$ to concentrate with our protein of interest. In order to thwart this problem in the future two different techniques were utilized, 10,000 MWCO dialysis into 1 $\times$ PBS and/or Detergent Removal Spin Columns.

The first ${ }^{15} \mathrm{~N}-\mathrm{HSQC}$ spectra for ${ }^{15} \mathrm{~N}-\alpha \mathrm{C}(233-425)$ were attained on a 16.4 T Varian NMR equipped with a triple resonance cryoprobe and confirmed on the 18 T Varian NMR (Figure 42). The concentration of $\alpha \mathrm{C}(233-425)$ was 210 $\mu \mathrm{M}$ and $\mathrm{pH}=6.6$. As this was an aqueous sample, Watergate was utilized for water suppression. These experiments prove that even at sub $\mathrm{mM}$ concentrations of aC $(233-425)$ one can still observe the ${ }^{1} \mathrm{H}-{ }^{15} \mathrm{~N}-\mathrm{HSQC}$ transitions. aC $(233-425)$ contains 193 residues, 17 of which are proline, so therefore there are 176 amide hydrogens. In addition to the amide backbone, 
there are 13 asparagines, 3 glutamines (each with two proton signals for the same nitrogen) and 7 tryptophans. Therefore, there should be a total of 199 observable ${ }^{15} \mathrm{~N}-{ }^{1} \mathrm{H}$ signals. The $\alpha \mathrm{C}(233-425){ }^{15} \mathrm{~N}-\mathrm{HSQC}$ successfully identified approximately 184 of the 199 possible signals (Appendix F). Of the $7 \operatorname{Trp}$ all but one was visible and 13 of the 19 Asn and Gln were visible.

Once an HSQC for aC $(233-425)$ was established, the next goal was to observe $\mathrm{aC}(233-425)$ in the presence of FXIII-A $\mathrm{A}_{2}$ (Figure 43). The same 210 $\mu \mathrm{M} \alpha \mathrm{C}(233-425)$ sample used previously was utilized to solubilize $3.2 \mathrm{mg}$ FXIII-A $A_{2}$. The sample precipitated out of solution and sonication for $5 \mathrm{~min}$ at

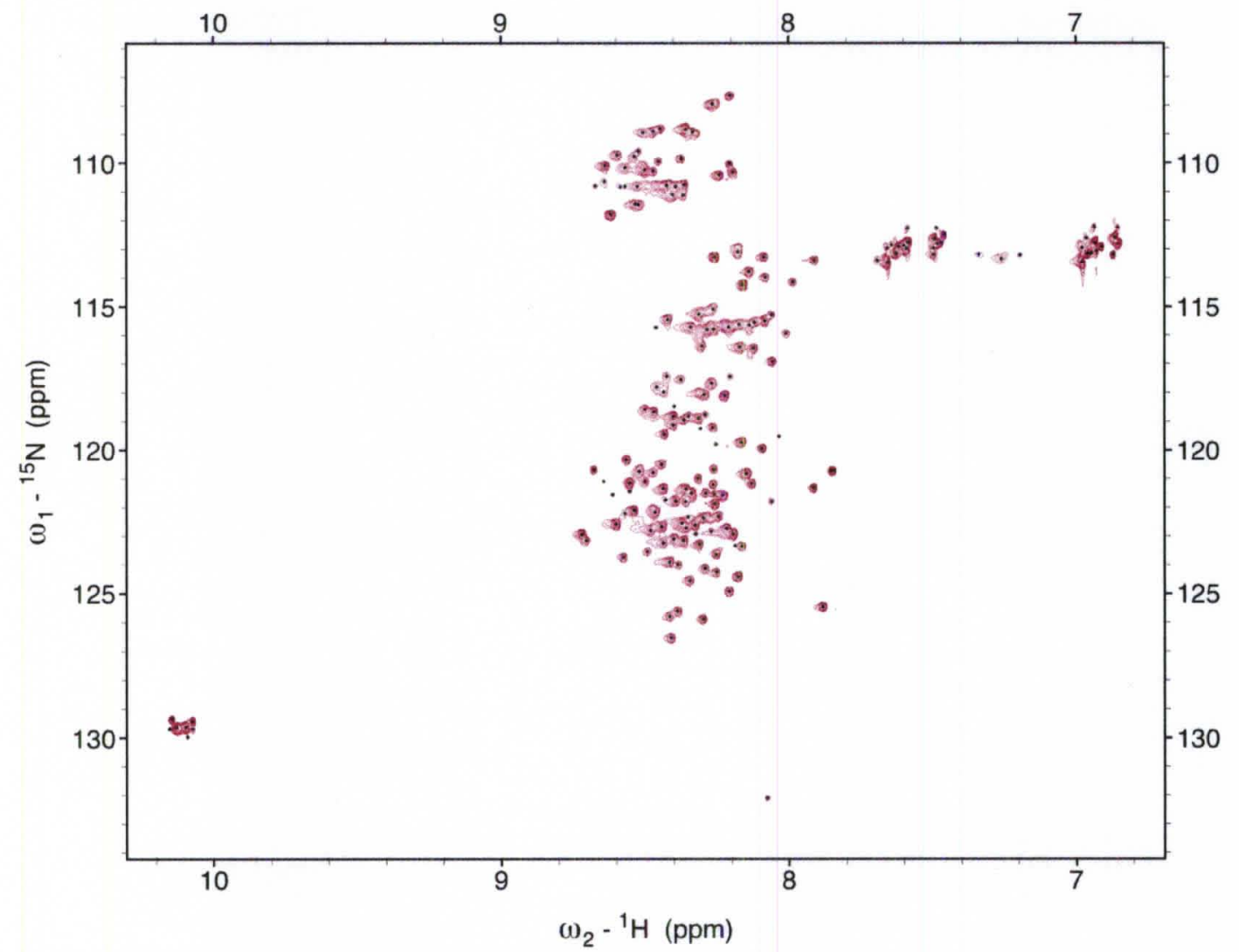

Figure 43: ${ }^{15} \mathrm{~N}-\mathrm{HSQC}$ spectra of ${ }^{15} \mathrm{~N}-\alpha \mathrm{C}(233-425)$ in the presence of FXIII- $\mathrm{A}_{2}$ conducted at $20{ }^{\circ} \mathrm{C}$ on an $800 \mathrm{MHz}(18 \mathrm{~T})$ Varian Inova NMR. The assignments can be viewed in Appendix G. 
room temperature did not increase solubility. The $\mathrm{pH}$ of the $\mathrm{aC}(233-425)-$ FXIII- $A_{2}$ solution was confirmed to be 6.6 and was spun at $14,000 \mathrm{rpm}$. The supernatant was then transferred to a Shigemi tube for NMR analysis. Due to precipitation and analysis of the supernatant the final concentration of $\mathrm{aC}$ and FXIII- $A_{2}$ was unknown, but assumed to be less than the starting concentrations $(210 \mu \mathrm{M}$ and $110 \mu \mathrm{M}$ respectively). The structure of $\alpha \mathrm{C}(233-425)$ in the presence of FXIII-A $A_{2}$ was also investigated using an $18 \mathrm{~T}$ Varian NMR. When aligned with the ${ }^{15} \mathrm{~N}-\alpha \mathrm{C}(233-425){ }^{1} \mathrm{H}-{ }^{15} \mathrm{~N}-\mathrm{HSQC},{ }^{15} \mathrm{~N}-\alpha \mathrm{C}(233-425)$ with FXIII$A_{2}$ did not exhibit any major peak shifts but several new peaks appeared. In the HSQC for $\alpha \mathrm{C}(233-425)$ in the presence of FXIII-A $A_{2}$, approximately 191 of the 199 possible $\mathrm{N}-\mathrm{H}$ stretches were observed (Appendix $\mathrm{G}$ ). This was an increase of 7 over the 184 signals observed for $\alpha \mathrm{C}(233-425)$. The most notable new peaks are at $(\mathrm{N}-120.7 \mathrm{ppm}, \mathrm{H}-8.68 \mathrm{ppm})$ and $(\mathrm{N}-121.8 \mathrm{ppm}, \mathrm{H}-8.36 \mathrm{ppm})$ as well as the Trp residue ( $\mathrm{N}-129.7 \mathrm{ppm}, \mathrm{H}-10.15 \mathrm{ppm})$. It is quite promising to see that even under conditions where some material may have been lost during precipitation, $\alpha C(233-425)$ still appears to gain some structure when in the presence of FXIII-A . . 


\section{Discussion}

Many kinetic and conformational dynamic studies require specific mutations. Thus it is imperative to establish and maintain functional expression systems. The $\mathrm{aC}(233-425) \mathrm{DNA}$ that was given to our lab has now been successfully transformed into both $\mathrm{DH} 5 \alpha$ and BL21 DE3 (gold) E. coli cells. The

transformed DNA was then utilized to express and purify aC $(233-425)$ for ${ }^{15} \mathrm{~N}$ HSQC NMR analysis.

$\alpha C(233-425)$ Expression

The DNA utilized had the $\alpha C(233-425)$ gene cloned into a pGEX-6P-1 vector. The pGEX-6P-1 vector has several benefits. Expression of $\alpha \mathrm{C}(233-$ $425)$ in the pGEX-6P-1 vector is induced with IPTG. aC $(233-425)$ expression is under the control of the tac promoter which is induced by IPTG, a lactose mimic. The pGEX-6P-1 plasmid also contains a $/ a c l^{q}$ gene which produces a repressor for the tac gene inhibiting expression until IPTG is added. To ease in protein purification following expression, the protein of interest is co-expressed with a GST tag. The GST tag is then subsequently utilized in affinity chromatography to capture the GST- $\alpha$ C product. Another benefit of the pGEX$6 \mathrm{P}-1$ is that it contains an Ampicillin resistance gene to assist in confirming transformation in your cells of interest.

Two different cell lines were chosen for $\alpha \mathrm{C}$ expression, the first of which was DH5a. The DH5a cells chosen were the Subcloning Efficiency Competent 
Cells which allowed for an efficient transformation and subsequent production of purified DNA for future transformations. One lesson learned during this process was that if the transformation does not produce nice round colonies and the plates are covered with a lawn of $E$. coli, the Ampicillin may have expired. According to Ryan, et al. Ampicillin starts to lose activity after 30 days at $4{ }^{\circ} \mathrm{C}$ (125). Following expression in DH5a cells the DNA that had been successfully purified was transformed into BL21 cells for aC $(233-425)$ expression and purification.

The E. coli strain BL21 gold (DE3) is frequently used as a host for protein expression studies. Bhandari and Gowrishankar reported on the robustness of the BL21 gold expression options (126). BL21 cells also limit the degradation of expressed proteins because they naturally lack genes for the proteases lon and ompT (127). Although transformation efficiency was never calculated, a nice selection of colonies was always observed. The reported efficiency for pUC18 DNA is $\geq 1 \times 10^{8} \mathrm{cfu}$ (colony forming units)/ $/ \mathrm{gg}$ of DNA (Stratagene, La Jolla, CA).

Once transformed into the BL21 gold cells, selected colonies were then grown in liquid LB broth to create glycerol stocks of cells ready for aC (233425) expression. The glycerol stocks were stored at $-70^{\circ} \mathrm{C}$ and a sterile loop was utilized to seed cultures for expression. After an overnight incubation, the starter culture was utilized to seed a larger (1-2 $L)$ culture. There are several variables which are critical to maximizing the amount of aC $(233-425)$ expressed, one of which is the proper cell density for induction. During log phase, the doubling time of E.coli is typically between $20-30$ min in nutrient rich 
media. It is during this time that cells are in the optimal condition for expressing

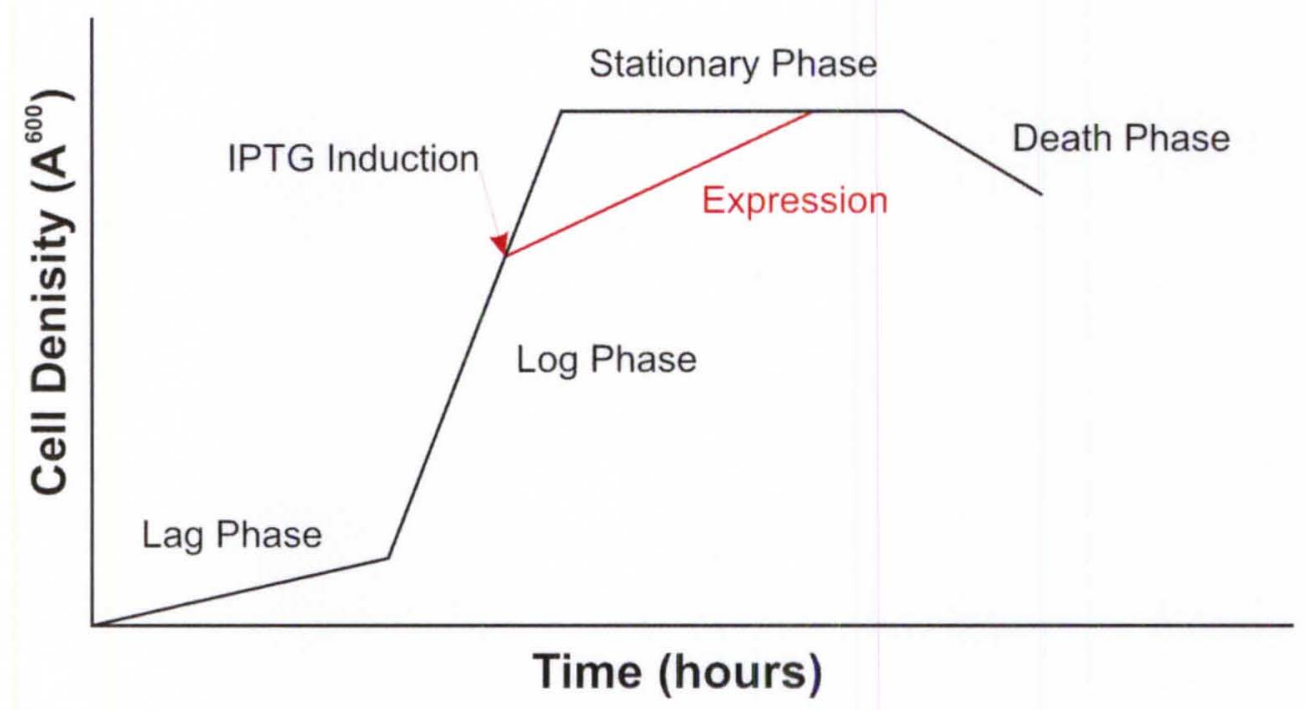

Figure 44: Theoretical $E$. coli growth curve. Lag phase displays the slow growth after inoculation. During Log Phase, the cell doubling time is around $20-30 \mathrm{~min}$. This is the optimal time for induction of protein expression. During stationary phase, nutrients are limited, cells become stressed and protein degradation can occur. During death phase cells begin to die due to lack of nutrients and cell density decreased over time.

protein (Figure 41). During LB broth, nutrient rich, expressions the cells were induced at $A^{600}=1.0$ and 0.6 for the minimal media expressions. The slope during log phase decreased from $0.54 \mathrm{~A}^{600}$ /hour for growth in nutrient rich media to $0.20 \mathrm{~A}^{600}$ /hour in minimal media. This $>2.5$ fold decrease in slope led to much longer incubation times prior to induction for the minimal media expressions.

To ensure induction during log phase, the induction for minimal media took place at $A^{600}=0.6$ since nutrient rich growth appears to be approaching stationary phase when $A^{600}=1.0$ (Figure 42). After IPTG addition both nutrient rich and minimal media cultures were allowed to incubate overnight at $30^{\circ} \mathrm{C}$. This is another variable which could possibly be optimized. By decreasing the rpm of the shaker from 225 to $180 \mathrm{rpm}$ and decreasing the temperature from 37 
${ }^{\circ} \mathrm{C}$ to $30{ }^{\circ} \mathrm{C}$, it slows the growth. It may be worth investigating shorter induction periods at $37^{\circ} \mathrm{C}$ and $225 \mathrm{rpm}$. The last issue concerning expression which was

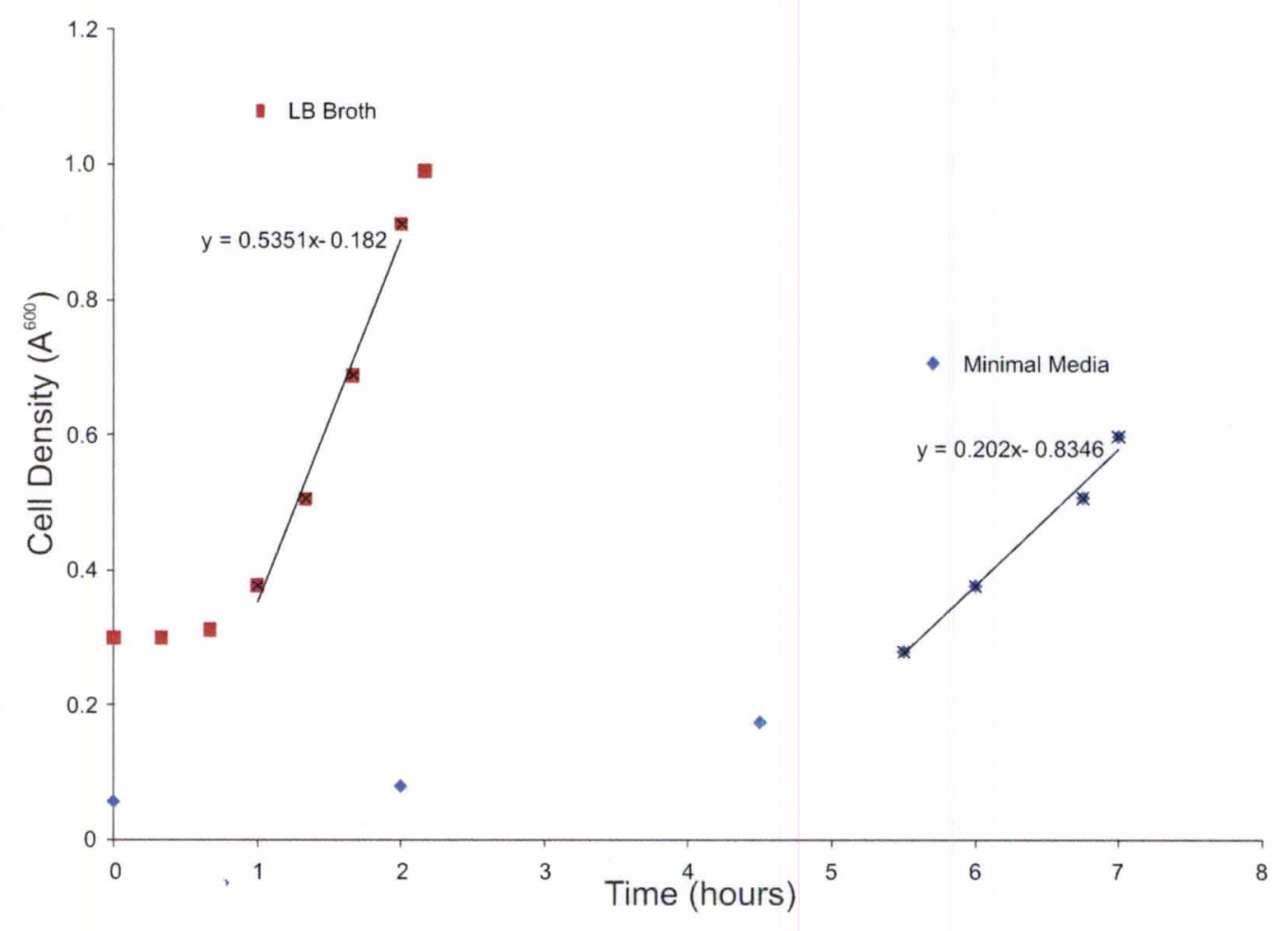

Figure 45: Representative growth curves for BL21 gold (DE3) cells grown in LB broth and minimal media. LB Broth is represented by the (Red Squares) and the time points utilized for calculating the slope of the Log Phase are marked with a black $(X)$. The minimal media growth curve is represented by (blue diamonds) and the time points utilized for calculating the slope of the Log Phase are marked with a black $(\mathrm{X})$.

overcome involved the use of Triton-100 during the purification process. When viewing the first $\alpha \mathrm{C}(233-425)$ NMR Triton X100 was the only molecule visible. The Triton X100 was concentrated when using 10,000 MWCO centrifuge concentrators to purify the aC $(233-425)$. To thwart this in future aC $(233-$ 425) expressions, both detergent removal spin columns and dialysis were utilized successfully. Further experimentation could be performed to determine which process is the most effective.

$\alpha C(233-425){ }^{15} \mathrm{~N}-\mathrm{HSQC}$ 
It was first reported in 1981 that the 242-424 region of the fibrinogen $\alpha$ chain was involved in reducing the concentration of $\mathrm{Ca}^{2+}$ needed for $\mathrm{FXIII-A}$ to dissociate from FXIII-B $B_{2}(74)$. Until recently very little research has been dedicated to further investigating this interaction between the aC-domain (Cterminal portion of the fibrin $\alpha$-chain) and FXIII-A. There have been a series of manuscripts which have focused on determining whether bovine and/or human aC-domain contains compact structure $(102,113,128)$. Burton et al. utilized NMR to identify and characterize a $\beta$-hairpin with a disulfide (C423-C453) restricting the base in bovine aC $(374-538)(102)$. The $\beta$-hairpin was then further characterized with a shorter aC fragment (406 - 483) focusing in on the compact structure (113). During this study aC (406 - 483) was fully characterized by NMR which allowed for the identification of a second loose $\beta$ hairpin that is formed by residues $459-476$. It was also determined by size exclusion chromatography and analytical ultracentrifugation that the $\mathrm{aC} \mathrm{(406-}$ 483) fragment formed oligomers in a concentration dependant manner, starting at concentrations as low as $3 \mathrm{mg} / \mathrm{ml}(113)$.

More recently, the knowledge gained from investigating the structure of bovine $\mathrm{aC}$ has been utilized to better understand human $\mathrm{\alpha C}$ structure. It was determined that human $\mathrm{aC}(425-503)$ and $(392-610)$ form oligomers as a function of concentration like their bovine counterparts (128). The NMR analysis of human aC (425 - 503), equivalent to bovine $(406-483)$, was limited due to the oligomerization. Since bovine $\alpha \mathrm{C}(406-483)$ had been fully characterized, the human $a \mathrm{C}{ }^{15} \mathrm{~N}-\mathrm{HSQC}$ was compared to the bovine. This comparison 
revealed that human $\alpha \mathrm{C}(425-503)$ contained several of the same peakes that were essential for $\beta$-hairpin formation in bovine $\alpha \mathrm{C}(113,128)$.

These human $\mathrm{aC}$ structural studies utilizing NMR have yet to describe how aC interacts with FXIII. Smith et al. has extensively studied the human aC $(233-425)$ fragment and how it interacts with unactivated FXIII-A, activated FXIII- $A_{2}$ and FXIII- $A_{2} B_{2}$ (121). Surface plasmon resonance (SPR) was used to study a series of truncated $\mathrm{aC}$ fragments and it was determined that activated

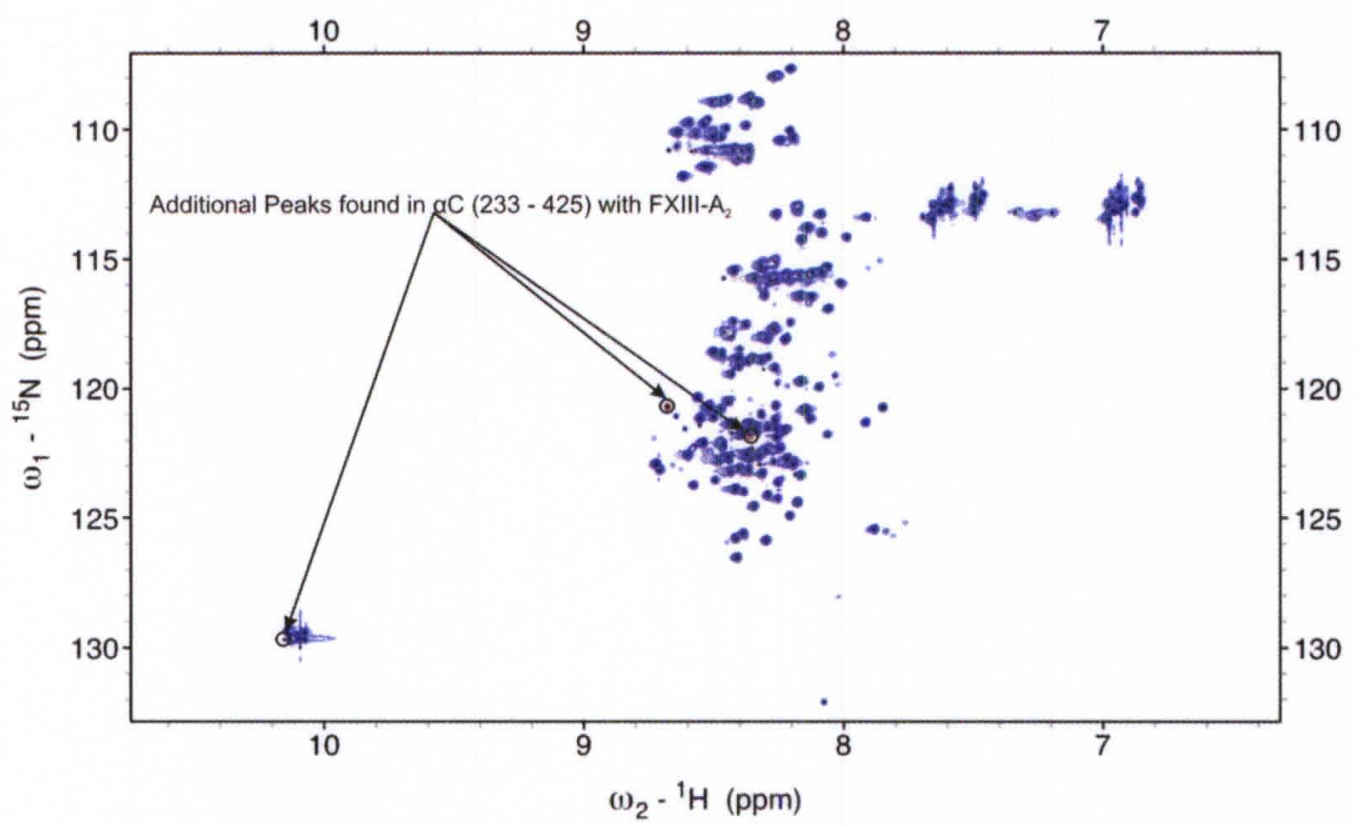

Figure 46: ${ }^{15} \mathrm{~N}-\mathrm{HSQC}$ spectra of ${ }^{15} \mathrm{~N}-\mathrm{aC}(233-425)$ overlaid on the spectra for ${ }^{15} \mathrm{~N}-\mathrm{aC}(233-425)$ in the presence of FXIII-A $\mathrm{A}_{2}$. The ${ }^{15} \mathrm{~N}-\mathrm{HSQC}$ spectrum for $\mathrm{aC}(233-425)$ is displayed in (blue) and $\mathrm{aC}(233-425)$ in the presence of FXIII- $A_{2}$ in (pink). The three peaks noted are only visible in the ${ }^{15} \mathrm{~N}-\mathrm{HSQC}$ spectra for aC (233-425) with FXIII-A $A_{2}$. The image was produced using the overlay command within Sparky 3 (124).

FXIII- $A_{2}$ had low $\mu \mathrm{M}$ affinity for $\mathrm{aC}$ and it was localized to residues $389-403$.

FXIII- $A_{2} B_{2}$ was found to have low $n M$ affinity and surprisingly, FXIII- $A_{2}$ zymogen did not bind to any of the $\mathrm{aC}$ fragments (121). The ${ }^{15} \mathrm{~N}-\mathrm{HSQC}$ data reported here investigates $\mathrm{aC}(233-425)$ both in the presence and absence of FXIII- $\mathrm{A}_{2}$ zymogen. After the addition of $\alpha \mathrm{C}$ to the lyophilized FXIII- $\mathrm{A}_{2}$ there was a 
substantial amount of precipitate formed. It is unknown whether the precipitate correlates to aC oligomerization or possible aC-FXIII- $\mathrm{A}_{2}$ interactions. The sample for aC with FXIII-A ${ }_{2}^{15} \mathrm{~N}-\mathrm{HSQC}$ analysis had to be spun down and only the supernatant was analyzed. The increase in total peaks identified for the $\mathrm{aC}$ $(233-425)$ with FXIII-A $A_{2}$ as seen in the overlay of the two spectra, alludes to an interaction that induces structure in $\mathrm{aC}$ (Figure 46). Our lab has now established an $\mathrm{aC}(233-425)$ expression system in standard media as well as minimal media capable of producing enough protein for NMR analysis. These NMR studies have now opened the door to further investigation of $\alpha \mathrm{C}(233-425)$ in the presence of FXIII-A2. 


\section{CHAPTER VI}

\section{CONCLUSIONS AND FUTURE DIRECTIONS}

Factor XIII is one of the last enzymes involved in the blood coagulation cascade and is responsible for cross-linking fibrin monomers to form an insoluble clot. Through the utilization of HDX coupled with MALDI-TOF MS, great strides have been made in clarifying FXIII- $A_{2}$ conformational dynamics during activation. The importance of FXIII during haemostasis has led to increased interest in acquiring a greater understanding of how this enzyme interacts with its substrates and cofactors in solution. Therefore, in addition to studying FXIII, NMR was utilized to investigate the FXIII- $A_{2}$ substrate fibrin aC (233-425).

The conformational dynamics of Factor XIII-A $\mathrm{A}_{2}$ (zymogen, FXIIIalla, $\mathrm{FXIIIa}^{\mathrm{Ca}}$, and inhibited with K9-Don) in solution has been extensively studied using $\operatorname{HDX}(43,45-46)$. One area that lacked understanding was how the FXIII$\mathrm{A}_{2}$ structure was dependent upon physiological $\mathrm{Ca}^{2+}$ concentrations. Chapter II describes the first objective of this project; to investigate the conformational

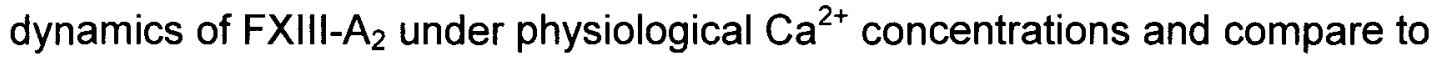
other metals of interest $\left(\mathrm{Mg}^{2+}, \mathrm{Ba}^{2+}\right.$, and $\left.\mathrm{Cu}^{2+}\right)$. $\mathrm{HDX}$ analysis identified regions of $\mathrm{FXIII-A} \mathrm{A}_{2}$ where $\mathrm{Ca}^{2+}$ elicited unique conformational differences when compared to the other metals studied. There were also several regions of FXIII-A $A_{2}$ where all metals examined yielded similar conformational changes. Due to the similarities 
observed for these metals, they were then tested to determine whether they could promote non -proteolytic FXIII-A $A_{2}$ activity.

In addition to studying FXIII- $\mathrm{A}_{2}$ non-proteolytic activation in the presence of $50 \mathrm{mM} \mathrm{Ca}^{2+}, \mathrm{Mg}^{2+}, \mathrm{Ba}^{2+}$ and $\mathrm{Cu}^{2+}$, we also investigated $500 \mathrm{mM} \mathrm{Na}^{+}$and two organic cations $\mathrm{EDA}^{2+}$ and $\mathrm{TMAC}^{+}$. When investigating each cation individually only $50 \mathrm{mM} \mathrm{Ca}^{2+}$ could promote FXIII-A $\mathrm{A}_{2}$ activity. The cations $\mathrm{Mg}^{2+}, \mathrm{Ba}^{2+}, \mathrm{Na}^{+}$,

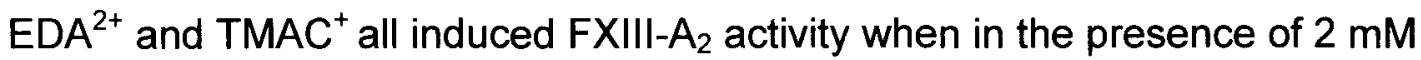
$\mathrm{Ca}^{2+}$. Low $\mathrm{mM} \mathrm{Ca}^{2+}$ was essential for non-proteolytic activation. The activity observed using organic cations $\left(\mathrm{EDA}^{2+}\right.$ and $\mathrm{TMAC}^{+}$) revealed that non-proteolytic activation is not unique to metal ions (44). HDX analysis was then utilized to study the conformational dynamics of non-proteolytically activated FXIII-A2. These analyses revealed that exposure around the active site and substrate recognition regions was initiated by physiological $\mathrm{Ca}^{2+}$ concentrations and set the stage for the larger conformational changes observed after activation.

While working on the FXIII-A $\mathrm{A}_{2}$ conformational dynamics presented in Chapter II, Pinkas et al. reported that TG2 is trapped in an open conformation when bound to a substrate-based inhibitor (49). It has been debated whether FXIII- $A_{2}$ undergoes a similar conformational change; therefore, in Chapter III HDX was utilized to compare the solvent accessibility of TG2 zymogen to that of activated TG2 and also inhibited TG2. Evidence of the open conformation was observed as increased exposure on the $\beta$-barrels and regions of the catalytic core. When comparing FXIII-A $A_{2}$ to the open conformation of TG2 it becomes obvious that in order to exist in an open conformation, FXIII- $\mathrm{A}_{2}$ must overcome 
substantial steric strain between the $\beta$-sandwich of one FXIII-A $A_{2}$ monomer and $\beta$ barrel 2 of the opposing monomer. If FXIII- $A_{2}$ does adopt an open conformation, it could lead to further developments in pharmaceuticals and antibodies specific for the open conformation which could be utilized in correcting aberrant FXIII-A during coagulation.

In regards to comparing TG2 and FXIII- $\mathrm{A}_{2}$ conformational dynamics, future work could take a few directions. Y238C FXIII, a physiological mutant, is a monomer. Now that our lab has established expression systems, it may be possible to express FXIII-A Y283C and compare FXIII-A in the monomer form to TG2. The Y283C monomer would be utilized because, as mentioned in Chapter III, the 'open' conformation seen in TG2 may be sterically impossible for the FXIII-A $A_{2}$ dimer. Although the Y283C mutant is physiological, it is not stable in whole plasma, having a shorter $t_{1 / 2}$ than wild type FXIII (99). If the Y283C mutant shows promise, mutation of the homologous TG2 residues may create a stable monomer, which one day might be used as a therapeutic for FXIII deficiency and thus combat postoperative bleeding

After exploring FXIII- $\mathrm{A}_{2}$ during activation and comparing FXIII- $\mathrm{A}_{2}$ to the open versus closed conformation for TG2, our focus shifted in Chapter IV to the conformational dynamics of FXIII-A $A_{2}$ bound to $F X I I I-B_{2}$ but before activation. It has been proposed that the two $\beta$-barrels of FXIII- $A_{2}$ interact with FXIII- $B_{2}$ in the heterotetramer since heterotetramer formation does not occur when the $\beta$-barrels are truncated (99). This evidence is supported by the significant protection from HDX observed in the $\beta$-barrels. If the FXIII- $B_{2}$ dimer assumes the structure 
proposed by Souri, et al. its length would be $>380 \AA$ (98). The FXIII-A $A_{2}$ structure is only $102 \AA$ in length, and therefore, protection was observed throughout FXIII$A_{2}$. One FXIII-A $A_{2}$ region of interest during activation, the dimer interface, becomes significantly protected when bound to FXIII- $\mathrm{B}_{2}$, which would limit FXIII$A_{2}$ activation.

To further investigate the FXIII- $A_{2} B_{2}$ heterotetramer, a more intensive HDX method could be performed. Our MALDI-TOF MS method works well, but increased sensitivity and increased sequence coverage is observed using LCICR-MS (129-130). Develop an HDX method on an LC-FT-ICR-MS would open many new doors. Historically, LC methods were avoided during HDX experimentation due to increased hydrogen back-exchange during the aqueous separation prior to MS detection. This problem is now avoided by keeping the column and transfer lines chilled to $0^{\circ} \mathrm{C}$ and also the advances in $\mathrm{LC}$ and column technology that have led to shorter run times $(1 \mathrm{~min})$. One factor limiting HDX with MALDI-TOF-MS detection is the mass of the protein and number of proteins being analyzed due to the increase in peptides produced and the inability to distinguish them. A liquid chromatography separation of peptides prior to MS detection allows for the identification of peptides of similar mass that overlap in a MALDI-MS.

Now that the regions of FXIII-A $A_{2}$ which contact FXIII- $B_{2}$ in the FXIII-A $A_{2} B_{2}$ heterotetramer have been established, it would be of interest to utilize NMR to determine the regions of $\alpha \mathrm{C}(233-425)$ that contact on $\mathrm{FXIII-} \mathrm{A}_{2} \mathrm{~B}_{2}$. The $\alpha \mathrm{C}(233$ -425 ) region is involved in the dissociation of FXIII-A $A_{2}$ and FXIII- $B_{2}$, and may 
adopt a unique conformation when interacting with the $F X I I I-A_{2} B_{2}$ heterotetramer. It would be nice to compare the analysis of $\mathrm{aC}(233-425)$ in the presence of FXIII- $A_{2} B_{2}$ to the results reported in chapter $V$ for $\alpha C(233-425)$ in the presence of FXIII-A .

Lastly, in chapter V, a method was established for expressing aC (233 425 ) in both standard LB broth for bulk protein production and minimal media for isotopically labeling the protein to enable NMR analysis. The $\mathrm{aC}(233-425)$ domain was chosen because of its known involvement in fibrin crosslinking by FXIII-A $A_{2}$. Not only does $\alpha \mathrm{C}(233-425)$ contain 3 FXIII-A $A_{2}$ Gln substrate residues, it also acts as a cofactor in the dissociation of $\mathrm{FXIII-A} \mathrm{A}_{2} \mathrm{~B}_{2}$ as well $(74$, 109). The $a C(233-425)$ region is largely unstructured and has yet to be visualized via crystallography (110-111).

In an attempt to determine if the $\alpha \mathrm{C}(233-425)$ region adopts a compact structure when interacting with FXIII- $\mathrm{A}_{2},{ }^{15} \mathrm{~N}-\alpha \mathrm{C}(233-425)$ was expressed in minimal medial and subjected to ${ }^{15} \mathrm{~N}-\mathrm{HSQC}$ NMR analysis. An increase in the total number of peaks indicates that the interaction between $\alpha \mathrm{C}(233-425)$ and FXIII- $A_{2}$ induces a more structured $\alpha C$. While these NMR studies were being developed, Smith et al. used SPR techniques, to demonstrate that $\alpha \mathrm{C}$ (233425) has low $n M$ affinity for $F X I I I-A_{2} B_{2}$ and FXIIla but does not interact with FXIII$A_{2}$ zymogen (121). These results lead to the question of whether $\alpha \mathrm{C}(233-425)$ adopts a more defined structure when interacting with activated FXIII- $\mathrm{A}_{2}$ or FXIII$A_{2} B_{2}$ versus the $F X I I I-A_{2}$ analyzed. 
Once an $\alpha \mathrm{C}(233-425)$ compact structure is defined, several different heteronuclear methods will be used to assign the HSQC spectrum. TOCSY $-{ }^{15} \mathrm{~N}$ $\mathrm{HSQC}$ can be run with a singly labeled ${ }^{15} \mathrm{~N}$ - aC-domain. Further analysis could be conducted with doubly labeled ${ }^{13} \mathrm{C}-{ }^{15} \mathrm{~N}-\alpha \mathrm{C}-(233-425)$. The benefit of the doubly labeled protein is the ability to determine sequential residues using HNCO and $\mathrm{HN}(\mathrm{CA}) \mathrm{CO}$ pulse sequences. For assigning more atoms in the amino acid side chains, other 3D experiments such as: $H N C A, H N(C O) C A, H N(C A) C B$ and HN(COCA)CB can be used (131).

To determine the structure, NOE assignments could then be elucidated using HSQC-NOESY, ${ }^{15} \mathrm{~N}-{ }^{13} \mathrm{C}$ edited NOESY and ${ }^{13} \mathrm{C}-{ }^{13} \mathrm{C}$-edited NOESY. The residual dipolar couplings (RDCs) of the amide backbone could be determined using ${ }^{1} \mathrm{H}-{ }^{15} \mathrm{~N}$ IPAP-HSQC with and without Pfl phage for spin alignment (102). The NOE data gathered could then be entered into XPLOR-NIH (132) for structural calculations using the angle restraints calculated by TALOS (133). It

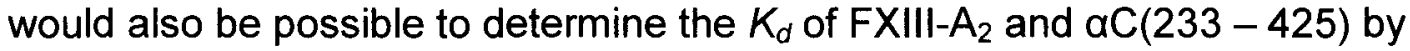
titrating in FXIII-A $\mathrm{A}_{2}$ and monitoring the shift in resonances for the residues that contact FXIII. TG2 could also be titrated in to determine if $\alpha \mathrm{C}(233-425)$ interacts with the two transglutaminases differently. This comparison would aid in identifying differences in TG2 and FXIII substrate specificity.

These studies have shed light upon the illusive FXIII conformational dynamics. This research presents a more global and functional understanding of how FXIII interacts with substrates and allosteric effectors like $\mathrm{Ca}^{2+}$ in solution. These discoveries may lead to better control of FXIII-A activity during 
thrombosis and atherosclerosis through a more knowledgeable route of drug discovery/design. 


\section{REFERENCES}

1. Voet, D. a. V., J.G. (1995) Biochemistry, Wiley and Sons, Inc., New York, NY.

2. Smith, S. A. (2009) The Cell-Based Model of Coagulation, Journal of Veterinary Emergency and Critical Care 19, 3-10.

3. Macfarlane, R. G. (1964) An Enzyme Cascade in the Blood Clotting Mechanism, and its Function as a Biochemical Amplifier, Nature 202, 498499.

4. Davie, E. W., and Ratnoff, O. D. (1964) Waterfall Sequence for Intrinsic Blood Clotting Science (New York, N.Y.) 145, 1310-1312.

5. Bolton-Maggs, P. H. B., and Pasi, K. J. (2003) Haemophilias A and B, Lancet 361, 1801-1809.

6. Hoffman, M., and Monroe, D. M., 3rd. (2001) A cell-based model of hemostasis, Thrombosis and Haemostasis 85, 958-965.

7. Martin, D. M., Boys, C. W., and Ruf, W. (1995) Tissue factor: molecular recognition and cofactor function, The FASEB Journal: Official Publication of the Federation of American Societies for Experimental Biology 9, 852859.

8. Monroe, D. M., Hoffman, M., and Roberts, H. R. (1996) Transmission of a procoagulant signal from tissue factor-bearing cell to platelets, Blood Coagulation \& Fibrinolysis: An International Journal in Haemostasis and Thrombosis 7, 459-464.

9. Díaz-Ricart, M., Estebanell, E., Lozano, M., Aznar-Salatti, J., White, J. G., Ordinas, A., and Escolar, G. (2000) Thrombin facilitates primary platelet adhesion onto vascular surfaces in the absence of plasma adhesive proteins: studies under flow conditions, Haematologica 85, 280-288.

10. Coughlin, S. R. (2001) Protease-activated receptors in vascular biology, Thrombosis and Haemostasis 86, 298-307.

11. Fay, P. J. (2006) Factor VIII structure and function, International Journal of Hematology 83, 103-108.

12. Oliver, J. A., Monroe, D. M., Roberts, H. R., and Hoffman, M. (1999) Thrombin activates factor $\mathrm{XI}$ on activated platelets in the absence of factor XII, Arteriosclerosis, Thrombosis, and Vascular Biology 19, 170-177.

13. Weisel, J. W. (2005) Fibrinogen and fibrin, Advances in Protein Chemistry 70, 247-299.

14. Lorand, L. (2001) Factor XIII: Structure, activation, and interactions with fibrinogen and fibrin, Annals of the New York Academy of Sciences 936, 291-311. 
15. Sakata, Y., and Aoki, N. (1980) Cross-Linking of Alpha-2-Plasmin Inhibitor to Fibrin by Fibrin-Stabilizing Factor, Journal of Clinical Investigation 65, 290-297.

16. Bajzar, L. (2000) Thrombin activatable fibrinolysis inhibitor and an antifibrinolytic pathway, Arteriosclerosis, Thrombosis, and Vascular Biology 20, 2511-2518.

17. Ichinose, A., and Aoki, N. (1982) Reversible Cross-Linking of Alpha-2Plasmin Inhibitor to Fibrinogen by Fibrin-Stabilizing Factor, Biochimica Et Biophysica Acta 706, 158-164.

18. DeLano, W. L. (2002) The PyMOL Molecular Graphics System, DeLano Scientific, San Carlos, CA.

19. Adany, R., Belkin, A., Vasilevskaya, T., and Muszbek, L. (1985) Identification of Blood-Coagulation Factor-Xiii in Human PeritonealMacrophages, European Journal of Cell Biology 38, 171-173.

20. Muszbek, L., Adany, R., Szegedi, G., Polgar, J., and Kavai, M. (1985) Factor-XIII of Blood-Coagulation in Human-Monocytes, Thromb. Res. 37, 401-410.

21. Adany, R., Glukhova, M. A., Kabakov, A. Y., and Muszbek, L. (1988) Characterization of Connective-Tissue Cells Containing Factor-Xiii Subunit-A, Journal of Clinical Pathology 41, 49-56.

22. Barkan G., G., A. (1923) Zur Frage der Reversibilitat der Fibringerinnung II, Biochem Ztschr 139, 291-301.

23. Lorand, L. (1950) Fibrin Clots, Nature 166, 694-695.

24. Muszbek, L., Yee, V. C., and Hevessy, Z. (1999) Blood coagulation factor XIII: Structure and function, Thromb. Res. 94, 271-305.

25. Radek, J. T., Jeong, J. M., Wilson, J., and Lorand, L. (1993) Association of the a-Subunits of Recombinant Placental Factor-Xiii with the Native Carrier B-Subunits from Human Plasma, Biochemistry 32, 3527-3534.

26. Schwartz, M. L., Pizzo, S. V., Hill, R. L., and Mckee, P. A. (1973) Human Factor-Xiii from Plasma and Platelets - Molecular-Weights, Subunit Structures, Proteolytic Activation, and Crosslinking of Fibrinogen and Fibrin, Journal of Biological Chemistry 248, 1395-1407.

27. Yee, V. C., Pedersen, L. C., Letrong, I., Bishop, P. D., Stenkamp, R. E., and Teller, D. C. (1994) 3-Dimensional Structure of a Transglutaminase Human Blood-Coagulation Factor-Xiii, Proceedings of the National Academy of Sciences of the United States of America 91, 7296-7300.

28. Hornyak, T. J., and Shafer, J. A. (1992) Interactions of Factor-Xiii with Fibrin as Substrate and Cofactor, Biochemistry 31, 423-429.

29. Naski, M. C., Lorand, L., and Shafer, J. A. (1991) Characterization of the Kinetic Pathway for Fibrin Promotion of Alpha-Thrombin-Catalyzed Activation of Plasma Factor-Xiii, Biochemistry 30, 934-941.

30. Chung, S. I., Lewis, M. S., and Folk, J. E. (1974) Relationships of Catalytic Properties of Human Plasma and Platelet Transglutaminases (Activated Blood-Coagulation Factor-Xiii) to Their Subunit Structures, Journal of Biological Chemistry 249, 940-950. 
31. Janus, T. J., Lewis, S. D., Lorand, L., and Shafer, J. A. (1983) Promotion of Thrombin-Catalyzed Activation of Factor-Xiii by Fibrinogen, Biochemistry 22, 6269-6272.

32. Hedner, U., Bergentz, S. E., Nilsson, I. M., Lunden, R., and Lorand, L. (1974) Experimental Studies on Inhibition of Factor-Xiii Invivo, Circulation 50, 294-294.

33. Curtis, C. G., Brown, K. L., Credo, R. B., Domanik, R. A., Gray, A., Stenberg, P., and Lorand, L. (1974) Calcium-dependent unmasking of active center cysteine during activation of fibrin stabilizing factor, Biochemistry 13, 3774-3780.

34. Pedersen, L. C., Yee, V. C., Bishop, P. D., Letrong, I., Teller, D. C., and Stenkamp, R. E. (1994) Transglutaminase Factor-XIII Uses ProteinaseLike Catalytic Triad to Cross-Link Macromolecules, Protein Science 3, 1131-1135.

35. Micanovic, R., Procyk, R., Lin, W., and Matsueda, G. R. (1994) Role of histidine 373 in the catalytic activity of coagulation factor XIII, The Journal of Biological Chemistry 269, 9190-9194.

36. Souri, M., Yee, V. C., Kasai, K., Kaneshiro, T., Narasaki, K., Castaman, G., and Ichinose, A. (2001) Novel Y283C mutation of the A subunit for coagulation factor XIII: molecular modelling predicts its impaired protein folding and dimer formation, British Journal of Haematology 113, 652-654.

37. Tamaki, T., and Aoki, N. (1981) Cross-Linking of Alpha-2-Plasmin Inhibitor and Fibronectin to Fibrin by Fibrin-Stabilizing Factor, Biochimica Et Biophysica Acta 661, 280-286.

38. Lorand, L., Credo, R. B., and Janus, T. J. (1981) Factor-Xiii (FibrinStabilizing Factor), Methods in Enzymology 80, 333-341.

39. Credo, R. B., Curtis, C. G., and Lorand, L. (1978) Ca2+-Related Regulatory Function of Fibrinogen, P Natl Acad Sci USA 75, 4234-4237.

40. Polgar, J., Hidasi, V., and Muszbek, L. (1990) Non-Proteolytic Activation of Cellular Protransglutaminase (Placenta Macrophage Factor-Xiii), Biochemical Journal 267, 557-560.

41. Yee, V. C., Pedersen, L. C., Bishop, P. D., Stenkamp, R. E., and Teller, D. C. (1995) Structural Evidence That the Activation Peptide Is Not Released Upon Thrombin Cleavage of Factor-XIII, Thromb. Res. 78, 389-397.

42. Fox, B. A., Yee, V. C., Pedersen, L. C., Le Trong, I., Bishop', P. D., Stenkamp, R. E., and Teller, D. C. (1999) Identification of the calcium binding site and a novel ytterbium site in blood coagulation factor XIII by X-ray crystallography, Journal of Biological Chemistry 274, 4917-4923.

43. Sabo, T. M., Brasher, P. B., and Maurer, M. C. (2007) Perturbations in factor XIII resulting from activation and inhibition examined by solution based methods and detected by MALDI-TOF MS, Biochemistry 46, 10089-10101.

44. Woofter, R. T., and Maurer, M. C. (2011) Role of calcium in the conformational dynamics of factor XIII activation examined by hydrogendeuterium exchange coupled with MALDI-TOF MS, Archives of Biochemistry and Biophysics 512, 87-95. 
45. Turner, B. T., and Maurer, M. C. (2002) Evaluating the roles of thrombin and calcium in the activation of coagulation factor XIII using H/D exchange and MALDI-TOF MS, Biochemistry 41, 7947-7954.

46. Andersen, M. D., and Faber, J. H. (2011) Structural characterization of both the non-proteolytic and proteolytic activation pathways of coagulation Factor XIII studied by hydrogen-deuterium exchange mass spectrometry, International Journal of Mass Spectrometry 302, 139-148.

47. Wales, T. E., and Engen, J. R. (2006) Hydrogen exchange mass spectrometry for the analysis of protein dynamics, Mass Spectrometry Reviews 25, 158-170.

48. Englander, S. W. (2006) Hydrogen exchange and mass spectrometry: A historical perspective, Journal of the American Society for Mass Spectrometry 17, 1481-1489.

49. Pinkas, D. M., Strop, P., Brunger, A. T., and Khosla, C. (2007) Transglutaminase 2 Undergoes a Large Conformational Change upon Activation, PLoS Biol 5, e327.

50. Weiss, M. S., Metzner, H. J., and Hilgenfeld, R. (1998) Two non-proline cis peptide bonds may be important for factor XIII function, FEBS Letters 423, 291-296.

51. Humphrey, W., Dalke, A., and Schulten, K. (1996) VMD: Visual molecular dynamics, Journal of Molecular Graphics 14, 33-38.

52. Muszbek, L., Polgar, J., and Boda, Z. (1993) Platelet Factor-Xiii Becomes Active without the Release of Activation Peptide during Platelet Activation, Thrombosis and Haemostasis 69, 282-285.

53. Muszbek, L., Haramura, G., and Polgar, J. (1995) Transformation of Cellular Factor-Xiii into an Active Zymogen Transglutaminase in Thrombin-Stimulated Platelets, Thrombosis and Haemostasis 73, 702705.

54. Mary, A., Achyuthan, K. E., and Greenberg, C. S. (1988) B-Chains Prevent the Proteolytic Inactivation of the a-Chains of Plasma Factor-Xiii, Biochimica Et Biophysica Acta 966, 328-335.

55. Takahashi, N., Takahashi, Y., and Putnam, F. W. (1986) Primary Structure of Blood Coagulation Factor-XIIIA (Fibrinoligase, Transglutaminase) from Human Placenta Proceedings of the National Academy of Sciences of the United States of America 83, 8019-8023.

56. Lai, T. S., Achyuthan, K. E., Santiago, M. A., and Greenberg, C. S. (1994) Carboxyl-Terminal Truncation of Recombinant Factor-Xiii a-Chains Characterization of Minimum Structural Requirement for Transglutaminase Activity, Journal of Biological Chemistry 269, 24596-24601.

57. Mary, A., Achyuthan, K. E., and Greenberg, C. S. (1988) The Binding of Divalent Metal-Ions to Platelet Factor-Xiii Modulates Its Proteolysis by Trypsin and Thrombin, Archives of Biochemistry and Biophysics 261, 112121.

58. Hornyak, T. J., and Shafer, J. A. (1991) Role of Calcium-lon in the Generation of Factor-Xiii Activity, Biochemistry 30, 6175-6182. 
59. Hornyak, T. J., Bishop, P. D., and Shafer, J. A. (1989) Alpha-ThrombinCatalyzed Activation of Human-Platelet Factor-Xiii - Relationship between Proteolysis and Factor-Xiiia Activity, Biochemistry 28, 7326-7332.

60. Turner, B. T., Sabo, T. M., Wilding, D., and Maurer, M. C. (2004) Mapping of factor XIII solvent accessibility as a function of activation state using chemical modification methods, Biochemistry 43, 9755-9765.

61. Kristiansen, G. K., and Andersen, M. D. (2011) Reversible Activation of Cellular Factor XIII by Calcium, Journal of Biological Chemistry 286, 98339839.

62. Fickenscher, K., Aab, A., and Stuber, W. (1991) A Photometric Assay for Blood-Coagulation Factor-Xiii, Thrombosis and Haemostasis 65, 535-540.

63. Karpati, L., Penke, B., Katona, E., Balogh, I., Vamosi, G., and Muszbek, L. (2000) A modified, optimized kinetic photometric assay for the determination of blood coagulation factor XIII activity in plasma, Clinical Chemistry 46, 1946-1955.

64. Marinescu, A., Cleary, D. B., Littlefield, T. R., and Maurer, M. C. (2002) Structural features associated with the binding of glutamine-containing peptides to Factor XIII, Archives of Biochemistry and Biophysics 406, 920.

65. Mandell, J. G., Falick, A. M., and Komives, E. A. (1998) Identification of protein-protein interfaces by decreased amide proton solvent accessibility, Proceedings of the National Academy of Sciences of the United States of America 95, 14705-14710.

66. Mandell, J. G., Falick, A. M., and Komives, E. A. (1998) Measurement of amide hydrogen exchange by MALDI-TOF mass spectrometry, Analytical Chemistry 70, 3987-3995.

67. Sabo, T. M., Farrell, D. H., and Maurer, M. C. (2006) Conformational analysis of gamma ' peptide (410-427) interactions with thrombin anion binding exosite II, Biochemistry 45, 7434-7445.

68. Wang, L. T., Lane, L. C., and Smith, D. L. (2001) Detecting structural changes in viral capsids by hydrogen exchange and mass spectrometry, Protein Science 10, 1234-1243.

69. Chen, J. W., and Smith, D. L. (2001) Amide hydrogen exchange shows that malate dehydrogenase is a folded monomer at $\mathrm{pH} 5$, Protein Science 10, 1079-1083.

70. Lewis, B., Freyssinet, J-M., Holbrook, J. (1978) An Equilibrium Study of Metal Ion Binding to Human Plasma Coagulation Factor XIII, Biochem. J. 169, 397-402.

71. Achyuthan, K. E., Slaughter, T. F., Santiago, M. A., Enghild, J. J., and Greenberg, C. S. (1993) Factor-Xiiia-Derived Peptides Inhibit Transglutaminase Activity - Localization of Substrate Recognition Sites, Journal of Biological Chemistry 268, 21284-21292.

72. Mitkevich, O. V., Sobel, J. H., Shainoff, J. R., Vlasik, T. N., Kalantarov, G. F., Trakht, I. N., Streltsova, Z. A., and Samokhin, G. P. (1996) Monoclonal antibody directed to a fibrinogen A alpha\#529-539 epitope inhibits alpha- 
chain crosslinking by transglutaminases, Blood Coagulation \& Fibrinolysis 7, 85-92.

73. Mitkevich, O. V., Shainoff, J. R., DiBello, P. M., Yee, V. C., Teller, D. C., Smejkal, G. B., Bishop, P. D., Kolotushkina, I. S., Fickenscher, K., and Samokhin, G. P. (1998) Coagulation factor XIlla undergoes a conformational change evoked by glutamine substrate - Studies on kinetics of inhibition and binding of XIIla by a cross-reacting antifibrinogen antibody, Journal of Biological Chemistry 273, 14387-14391.

74. Credo, R. B., Curtis, C. G., and Lorand, L. (1981) Alpha-Chain Domain of Fibrinogen Controls Generation of Fibrinoligase (Coagulation Factor-Xiiia) - Calcium-Ion Regulatory Aspects, Biochemistry 20, 3770-3778.

75. Ichinose, A., and Davie, E. W. (1988) Characterization of the Gene for the a Subunit of Human Factor-Xiii (Plasma Transglutaminase), a BloodCoagulation Factor, Proceedings of the National Academy of Sciences of the United States of America 85, 5829-5833.

76. Ambrus, A., Banyai, I., Weiss, M. S., Hilgenfeld, R., Keresztessy, Z., Muszbek, L., and Fesus, L. (2001) Calcium binding of transglutaminases: A Ca-43 NMR study combined with surface polarity analysis, Journal of Biomolecular Structure \& Dynamics 19, 59-74.

77. Király, R., Csosz, E., Kurtán, T., Antus, S., Szigeti, K., Simon-Vecsei, Z., Korponay-Szabó, I. R., Keresztessy, Z., and Fésüs, L. (2009) Functional significance of five noncanonical $\mathrm{Ca} 2+$-binding sites of human transglutaminase 2 characterized by site-directed mutagenesis, The FEBS Journal 276, 7083-7096.

78. Ortner, E., Schroeder, V., Walser, R., Zerbe, O., and Kohler, H. P. (2010) Sensitive and selective detection of free FXIII activation peptide: a potential marker of acute thrombotic events, Blood 115, 5089-5096.

79. Cooke, R. D., Pestell, T. C., and Holbrook, J. J. (1974) Calcium and Thiol Reactivity of Human Plasma Clotting Factor-Xiii, Biochemical Journal 141, 675-682.

80. Brocklehurst, K., and Malthouse, J. P. (1978) Mechanism of the reaction of papain with substrate-derived diazomethyl ketones. Implications for the difference in site specificity of halomethyl ketones for serine proteinases and cysteine proteinases and for stereoelectronic requirements in the papain catalytic mechanism.

81. Nakaoka, H., Perez, D. M., Baek, K. J., Das, T., Husain, A., Misono, K., Im, M. J., and Graham, R. M. (1994) G(H) - a Gtp-Binding Protein with Transglutaminase Activity and Receptor Signaling Function, Science 264, 1593-1596.

82. Hausch, F., Halttunen, T., Mäki, M., and Khosla, C. (2003) Design, Synthesis, and Evaluation of Gluten Peptide Analogs as Selective Inhibitors of Human Tissue Transglutaminase, Chemistry \& Biology 10 , 225-231.

83. Cleary, D. B., and Maurer, M. C. (2006) Characterizing the specificity of activated factor XIII for glutamine-containing substrate peptides,

Biochimica Et Biophysica Acta-Proteins and Proteomics 1764, 1207-1217. 
84. Lorand, L., and Graham, R. M. (2003) Transglutaminases: Crosslinking enzymes with pleiotropic functions, Nature Reviews Molecular Cell Biology $4,140-156$.

85. Griffin, M., Casadio, R., and Bergamini, C. M. (2002) Transglutaminases: nature's biological glues, Biochemical Journal 368, 377-396.

86. Caccamo, D., Currò, M., and lentile, R. (2010) Potential of transglutaminase 2 as a therapeutic target, Expert Opinion on Therapeutic Targets 14, 989-1003.

87. Lai, T. S., Davies, C., and Greenberg, C. S. (2010) Human tissue transglutaminase is inhibited by pharmacologic and chemical acetylation, Protein Sci 19, 229-235.

88. Casadio, R., Polverini, E., Mariani, P., Spinozzi, F., Carsughi, F., Fontana, A., de Laureto, P. P., Matteucci, G., and Bergamini, C. M. (1999) The structural basis for the regulation of tissue transglutaminase by calcium ions, European Journal of Biochemistry 262, 672-679.

89. Cervellati, C., Franzoni, L., Squerzanti, M., Bergamini, C. M., Spinozzi, F., Mariani, P., Lanzara, V., and Spisni, A. (2009) Unfolding studies of tissue transglutaminase, Amino Acids 36, 633-641.

90. Stamnaes, J., Pinkas, D. M., Fleckenstein, B., Khosla, C., and Sollid, L. M. (2010) Redox regulation of transglutaminase 2 activity, The Journal of Biological Chemistry 285, 25402-25409.

91. Liu, S. P., Cerione, R. A., and Clardy, J. (2002) Structural basis for the guanine nucleotide-binding activity of tissue transglutaminase and its regulation of transamidation activity, Proceedings of the National Academy of Sciences of the United States of America 99, 2743-2747.

92. Turner, B. T., Sabo, T. M., Wilding, D., and Maurer, M. C. (2004) Mapping the solvent accessibility of Factor XIII as a function of activation state using chemical modification methods, Protein Science 13, 89-89.

93. KomÁRomi, I., Bagoly, Z., and Muszbek, L. (2011) Factor XIII: novel structural and functional aspects, Journal of Thrombosis and Haemostasis 9, 9-20.

94. Yorifuji, H., Anderson, K., Lynch, G., Van de Water, L., and McDonagh, J. (1988) B protein of factor XIII: differentiation between free B and complexed B, Blood 72, 1645-1650.

95. Carrell, N. A., Erickson, H. P., and Mcdonagh, J. (1989) ElectronMicroscopy and Hydrodynamic Properties of Factor-Xiii Subunits, Journal of Biological Chemistry 264, 551-556.

96. Ichinose, A., Bottenus, R. E., and Davie, E. W. (1990) Structure of transglutaminases, Journal of Biological Chemistry 265, 13411-13414.

97. Lozier, J., Takahashi, N., and Putnam, F. W. (1984) Complete Amino-Acid Sequence of Human-Plasma Beta-2 Glycoprotein I, Proceedings of the National Academy of Sciences of the United States of America-Biological Sciences 81, 3640-3644.

98. Souri, M., Kaetsu, H., and Ichinose, A. (2008) Sushi domains in the B subunit of factor XIII responsible for oligomer assembly, Biochemistry 47 , 8656-8664. 
99. Souri, M., and Ichinose, A. (2001) Impaired protein folding, dimer formation, and heterotetramer assembly cause intra- and extracellular instability of a Y283C mutant of the a subunit for coagulation factor XIII, Biochemistry 40, 13413-13420.

100. Greenberg, C. S., and Shuman, M. A. (1982) The Zymogen Forms of Blood-Coagulation Factor-Xiii Bind Specifically to Fibrinogen, Journal of Biological Chemistry 257, 6096-6101.

101. Schwarzenbacher, R., Zeth, K., Diederichs, K., Gries, A., Kostner, G. M., Laggner, P., and Prassl, R. (1999) Crystal structure of human beta2glycoprotein I: implications for phospholipid binding and the antiphospholipid syndrome, The EMBO Journal 18, 6228-6239.

102. Burton, R. A., Tsurupa, G., Medved, L., and Tjandra, N. (2006) Identification of an ordered compact structure within the recombinant bovine fibrinogen alpha C-domain fragment by NMRT, Biochemistry 45 , 2257-2266.

103. Mosesson, M. W., Siebenlist, K. R., and Meh, D. A. (2001) The Structure and Biological Features of Fibrinogen and Fibrin, Annals of the New York Academy of Sciences 936, 11-30.

104. Weisel, J., Stauffacher, C., Bullitt, E., and Cohen, C. (1985) A model for fibrinogen: domains and sequence, Science 230, 1388-1391.

105. Veklich, Y. I., Gorkun, O. V., Medved, L. V., Nieuwenhuizen, W., and Weisel, J. W. (1993) Carboxyl-terminal portions of the alpha chains of fibrinogen and fibrin. Localization by electron microscopy and the effects of isolated alpha $\mathrm{C}$ fragments on polymerization, Journal of Biological Chemistry 268, 13577-13585.

106. Weisel, J. W., and Medved, L. (2001) The Structure and Function of the alpha-C Domains of Fibrinogen, Annals of the New York Academy of Sciences 936, 312-327.

107. Medved, L. V., Gorkun, O. V., Manyakov, V. F., and Belitser, V. A. (1985) The Role of Fibrinogen alpha-C-Domains in the Fibrin Assembly Process, Febs Letters 181, 109-112.

108. Siebenlist, K. R., and Mosesson, M. W. (1992) Factors Affecting GammaChain Multimer Formation in Cross-Linked Fibrin, Biochemistry 31, 936941.

109. Shainoff, J. R., Urbanic, D. A., and Dibello, P. M. (1991) Immunoelectrophoretic Characterizations of the Cross-Linking of Fibrinogen and Fibrin by Factor-Xiiia and Tissue Transglutaminase Identification of a Rapid Mode of Hybrid Alpha-Chain Gamma-Chain Cross-Linking That Is Promoted by the Gamma-Chain Cross-Linking, Journal of Biological Chemistry 266, 6429-6437.

110. Kollman, J. M., Pandi, L., Sawaya, M. R., Riley, M., and Doolittle, R. F. (2011) Crystal Structure of Human Fibrinogen, Biochemistry 48, 3877 3886.

111. Madrazo, J., Brown, J. H., Litvinovich, S., Dominguez, R., Yakovlev, S., Medved, L., and Cohen, C. (2001) Crystal structure of the central region of bovine fibrinogen (E-5 fragment) at 1.4-angstrom resolution, Proceedings 
of the National Academy of Sciences of the United States of America 98, 11967-11972.

112. Tsurupa, G., Tsonev, L., and Medved, L. (2002) Structural organization of the fibrin(ogen) alpha C-domain, Biochemistry 41, 6449-6459.

113. Burton, R. A., Tsurupa, G., Hantgan, R. R., Tjandra, N., and Medved, L. (2007) NMR solution structure, stability, and interaction of the recombinant bovine fibrinogen alpha C-domain fragment, Biochemistry 46, 8550-8560.

114. Marley, J., Lu, M., and Bracken, C. (2001) A method for efficient isotopic labeling of recombinant proteins, Journal of Biomolecular NMR 20, 71-75.

115. Paliy, O., and Gunasekera, T. S. (2007) Growth of E. coli BL21 in minimal media with different gluconeogenic carbon sources and salt contents, Applied Microbiology and Biotechnology 73, 1169-1172.

116. Sambrook, J., Fritsch, E. F., and Maniatis, T. (1989) Molecular Cloning: $A$ Laboratory Manual, Cold Spring Harbor Laboratory Pr.

117. Cavanagh, J., Fairbrother, Wayne J., Palmer, Arthur G. III, Skelton Nicholas J. (1996) Protein NMR Spectroscopy: Principles and Practice, Academic Press, New York.

118. Dyson, H. J., and Wright, P. E. (2004) Unfolded proteins and protein folding studied by NMR, Chemical Reviews 104, 3607-3622.

119. Bodenhausen, G., and Ruben, D. J. (1980) Natural abundance nitrogen15 NMR by enhanced heteronuclear spectroscopy, Chemical Physics Letters 69, 185-189.

120. Dames, S. A., Martinez-Yamout, M., De Guzman, R. N., Dyson, H. J., and Wright, P. E. (2002) Structural basis for Hif-1a/CBP recognition in the cellular hypoxic response, Proceedings of the National Academy of Sciences 99, 5271-5276.

121. Smith, K. A., Adamson, P. J., Pease, R. J., Brown, J. M., Balmforth, A. J., Cordell, P. A., Ariëns, R. A. S., Philippou, H., and Grant, P. J. (2011) Interactions between factor $\mathrm{XIII}$ and the alphaC region of fibrinogen, $B L O O D$ 117, 3460-3468.

122. Biosciences, A. (2002) GST Gene Fusion System Handbook, Amersham Biosciences.

123. Delaglio, F., Grzesiek, S., Vuister, G., Zhu, G., Pfeifer, J., and Bax, A. (1995) NMRPipe: A multidimensional spectral processing system based on UNIX pipes, Journal of Biomolecular NMR 6.

124. Goddard, T. D., and Kneller, D. G. (2008) SPARKY 3, University of California, San Francisco.

125. Ryan, K. J., Needham, G. M., Dunsmoor, C. L., and Sherris, J. C. (1970) Stability of Antibiotics and Chemotherapeutics in Agar Plates, Applied Microbiology 20, 447-451.

126. Bhandari, P., and Gowrishankar, J. (1997) An Escherichia coli host strain useful for efficient overproduction of cloned gene products with $\mathrm{NaCl}$ as the inducer, Journal of Bacteriology 179, 4403-4406.

127. Yadava, R. S., Kumar, R., and Yadava, P. K. (2005) Expression of lexA targeted ribozyme in Escherichia coli BL-21 (DE3) cells, Molecular and Cellular Biochemistry 271, 197-203. 
128. Tsurupa, G., Hantgan, R. R., Burton, R. A., Pechik, I., Tjandra, N., and Medved, L. (2009) Structure, stability, and interaction of the fibrin(ogen) alphaC-domains, Biochemistry 48, 12191-12201.

129. Zhang, H.-M., Kazazic, S., Schaub, T. M., Tipton, J. D., Emmett, M. R., and Marshall, A. G. (2008) Enhanced digestion efficiency, peptide ionization efficiency, and sequence resolution for protein hydrogen/deuterium exchange monitored by Fourier transform ion cyclotron resonance mass spectrometry, Analytical Chemistry 80, 90349041.

130. Lanman, J., and Prevelige, P. E., Jr. (2004) High-sensitivity mass spectrometry for imaging subunit interactions: hydrogen/deuterium exchange, Current Opinion in Structural Biology 14, 181-188.

131. Rule, G. S., Hitchens, Kevin T. (2006) Fundamentals of Protein NMR Spectroscopy, Vol. 5, Springer, Netherlands.

132. Schwieters, C. D., Kuszewski, J. J., Tjandra, N., and Clore, G. M. (2003) The Xplor-NIH NMR molecular structure determination package, J Magn Reson 160, 65-73.

133. Cornilescu, G., Delaglio, F., and Bax, A. (1999) Protein backbone angle restraints from searching a database for chemical shift and sequence homology, J Biomol NMR 13, 289-302. 
APPENDIX A: A DETAILED DIAGRAM OUTLINING THE BLOOD COAGULATION CASCADE

Kindly provided by Abcam plc, Cambridge, England abcam

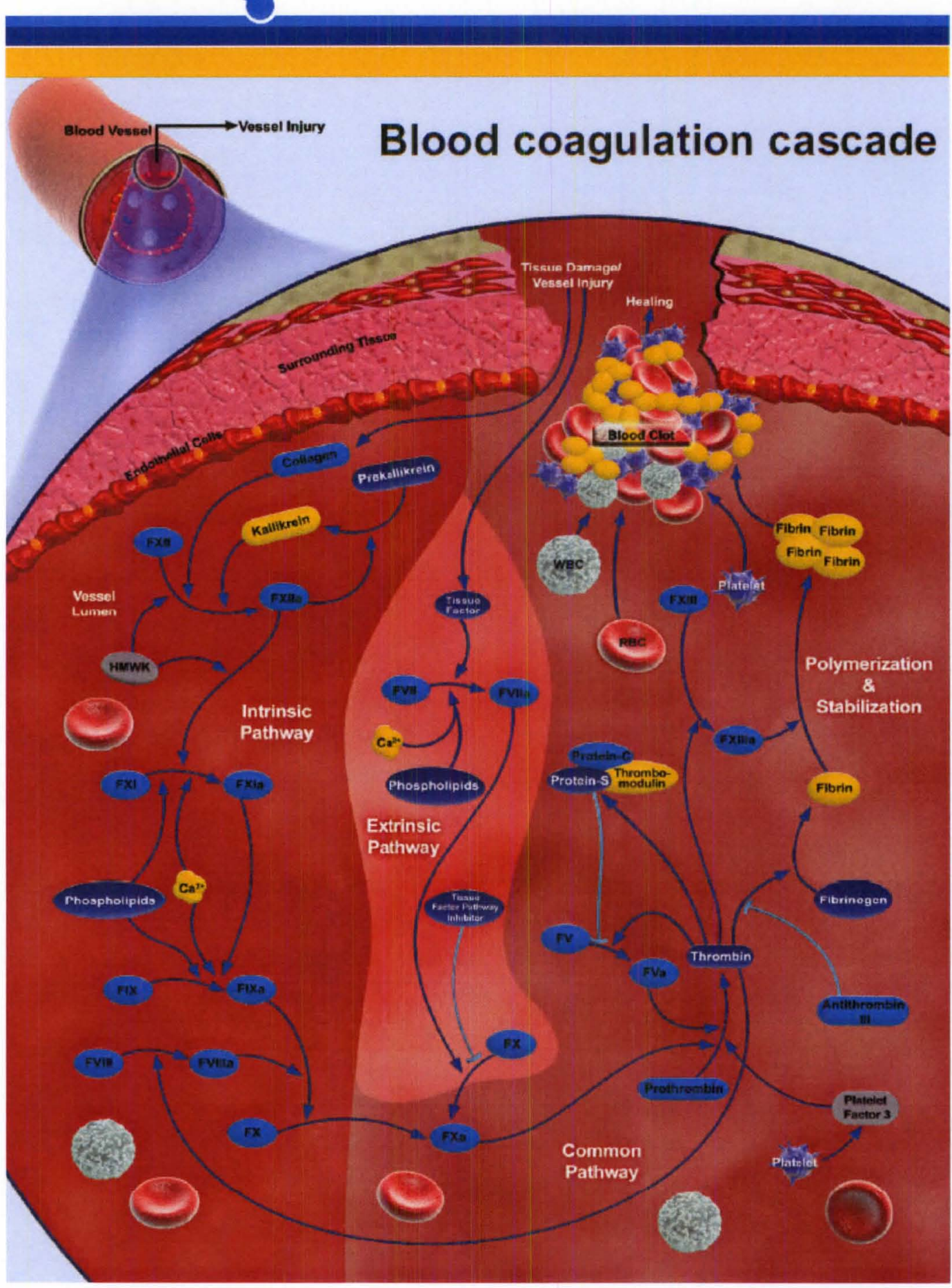


APPENDIX B: ABBREVIATIONS FOR BLOOD COAGULATION ENZYMES

\begin{tabular}{ll} 
Abbreviation & Enzyme \\
\hline II & Prothrombin \\
IIa & Thrombin \\
FV & Factor V \\
FVa & Activated Factor V \\
FVII & Factor VII \\
FVIla & Activated Factor VII \\
FVIII & Factor VIII \\
FVIIla & Activated Factor VIII \\
FIX & Factor IX \\
FIXa & Activated Factor IX \\
FX & Factor X \\
FXa & Activated Factor X \\
FXI & Factor XI \\
FXla & Activated Factor XI \\
FXII & Factor XII \\
FXIIa & Activated Factor XII \\
FXIII & Factor XIII \\
FXIIla & Activated Factor XIII \\
VWF & von Willebrand Factor
\end{tabular}


APPENDIX C: FXIII-A $A_{2}$ PEPTIC PEPTIDES

\begin{tabular}{|c|c|c|}
\hline Peptide & $\mathbf{m} / \mathbf{z}^{\mathbf{a}}$ & Amino Acid Sequence \\
\hline $1-23$ & 2431.30 & SETSRTAFGGRRAVPPNNSNAAE \\
\hline $32-40$ & 926.15 & QGVVPRGVN \\
\hline $53-74$ & 2819.06 & FKERWDTNKVDHHTDKYENNKL \\
\hline $75-82$ & 963.03 & IVRRGQSF \\
\hline $83-99$ & 2188.48 & YVQIDFSRPYDPRRDLF \\
\hline $88-98$ & 1422.78 & FSRPYDPRRDL \\
\hline $100-111$ & 1509.78 & RVEYVIGRYPQE \\
\hline $137-144$ & 1031.09 & REDRSVRL \\
\hline $145-159$ & 1682.03 & SIQSSPKCIVGKFRM \\
\hline $171-180$ & 1177.20 & RETSRNPETDT \\
\hline $194-208$ & 1930.10 & YLDNEKEREEYVLND \\
\hline $214-230$ & 2051.24 & YGEVNDIKTRSWSYGQF \\
\hline $220-230$ & 1372.70 & IKTRSWSYGQF \\
\hline $240-247$ & 1014.09 & YVMDRAQM \\
\hline $248-264$ & 1713.97 & DLSGRGNPIKVSRVGSA \\
\hline $248-265$ & 1845.15 & DLSGRGNPIKVSRVGSAM \\
\hline $298-305$ & 980.97 & ILLEYRSS \\
\hline $300-314$ & 1800.83 & LEYRSSENPVRYGQC \\
\hline $325-338$ & 1589.88 & LRCLGIPARIVTNY \\
\hline $328-338$ & 1217.36 & LGIPARIVTNY \\
\hline $364-372$ & 1126.21 & PVPIVSELQSG \\
\hline $380-389$ & 1133.28 & MTRPDLPVGF \\
\hline $407-424$ & 1972.96 & YRCGPASVQAIKHGHVCF \\
\hline $513-522$ & 1198.55 & KSRSNVDMDF \\
\hline $533-550$ & 2213.56 & FKLSITFRNNSHNRYTIT \\
\hline $535-541$ & 851.05 & LSITFRN \\
\hline $607-619$ & 1534.65 & FVTARINETRDVL \\
\hline $632-646$ & 1616.98 & IIIKVRGTQVVGSDM \\
\hline
\end{tabular}

a all $\mathrm{m} / \mathrm{z}$ values represent the average mass of the undeuterated cluster. 
APPENDIX D: TG2 PEPTIC PEPTIDES

\begin{tabular}{|c|c|l|}
\hline Peptide & $\mathbf{m}^{\mathbf{a}} \mathbf{z}^{\mathbf{a}}$ & \multicolumn{1}{|c|}{ Amino Acid Sequence } \\
\hline $12-25$ & 2431.30 & ELETNGRDHHTADL \\
\hline $26-39$ & 926.15 & CREKLVVRRGQPFW \\
\hline $31-39$ & 2819.06 & VVRRGQPFW \\
\hline $154-160$ & 963.03 & ERQEYVL \\
\hline $237-253$ & 2188.48 & LGRWDNNYGDGVSPMSW \\
\hline $259-269$ & 1422.78 & ILRRWKNHGCQ \\
\hline $315-329$ & 1509.78 & FRNEFGEIQGDKSEM \\
\hline $341-353$ & 1031.09 & MTRPDLQPGYEGW \\
\hline $369-377$ & 1682.03 & CCGPVPVRA \\
\hline $451-461$ & 1177.20 & EREAFTRANHL \\
\hline $452-461$ & 1930.10 & REAFTRANHL \\
\hline $475-487$ & 2051.24 & RIRVGQSMNMGSD \\
\hline $502-508$ & 1372.70 & VVCRLLL \\
\hline $547-554$ & 1014.09 & YEKYRDCL \\
\hline $560-568$ & 1713.97 & IKVRALLVE \\
\hline $668-686$ & 1845.15 & ESDKLKAVKGFRNVIIGPA \\
\hline
\end{tabular}

a all $\mathrm{m} / \mathrm{z}$ values represent the average mass of the undeuterated cluster. 
APPENDIX E: LB BROTH AND LB AGAR RECIPES

Table 1: $500 \mathrm{ml}$ LB Broth

$5.0 \mathrm{~g}$ Tryptone

$2.5 \mathrm{~g}$ Yeast Extract

$5.0 \mathrm{~g} \mathrm{NaCl}$

Add $500 \mathrm{ml} \mathrm{dH} \mathrm{H}_{2} \mathrm{O}$ and stir to mix. $\mathrm{pH}$ the solution to 7.5 with $10 \mathrm{M} \mathrm{NaOH}$ and autoclave before use. If Ampicillin is needed, add prior to inoculation.

Table 2: $500 \mathrm{ml}$ LB Agar

$5.0 \mathrm{~g}$ Tryptone

$2.5 \mathrm{~g}$ Yeast Extract

$5.0 \mathrm{~g} \mathrm{NaCl}$

$7.5 \mathrm{~g} \mathrm{Agar}$

Add $500 \mathrm{ml} \mathrm{dH}_{2} \mathrm{O}$ and stir to mix. $\mathrm{pH}$ the solution to 7.5 with $10 \mathrm{M} \mathrm{NaOH}$ and autoclave. Let cool to $\sim 50{ }^{\circ} \mathrm{C}$ and add $500 \mu \mathrm{l} 100 \mathrm{mg} / \mathrm{ml} \mathrm{Ampicillin.} \mathrm{Pour} \mathrm{plates}$ and allow them to cool at room temperature. Cap and store at $4{ }^{\circ} \mathrm{C}$.

Table 3: $1 \mathrm{~L}$ SOB Medium

$\begin{array}{ll}20.0 \mathrm{~g} & \text { Tryptone } \\ 5.0 \mathrm{~g} & \text { Yeast Extract }\end{array}$

$0.5 \mathrm{~g} \quad \mathrm{NaCl}$

Add $1 \mathrm{~L} \mathrm{dH} \mathrm{H}_{2} \mathrm{O}$ and autoclave. After the $\mathrm{SOB}$ solution has cooled, add $10 \mathrm{ml}$ filter sterilized $1 \mathrm{M} \mathrm{MgCl}_{2}$ and $10 \mathrm{ml}$ filter sterilized $1 \mathrm{M} \mathrm{MgSO}_{4}$.

Table 4: $1 \mathrm{~L}$ SOC Medium (make immediately before use)

Add $2 \mathrm{ml}$ filter sterilized $20 \%$ glucose solution to $100 \mathrm{ml} \mathrm{SOB}$ Medium.

Table 5: $1 \mathrm{~L}$ Phosphate Buffer

$23.2 \mathrm{~g} \quad \mathrm{KH}_{2} \mathrm{PO}_{4}$

$164 \mathrm{~g} \quad \mathrm{~K}_{2} \mathrm{HPO}_{4}$

Dilute to $1 \mathrm{~L}$ in volumetric flask. Transfer to autoclave safe bottle and autoclave.

Table 6: 2 L Terrific Broth

\begin{tabular}{ll}
\hline $24.0 \mathrm{~g}$ & Tryptone \\
$48.0 \mathrm{~g}$ & Yeast Extract \\
$8 \mathrm{ml}$ & Glycerol
\end{tabular}

$8 \mathrm{ml} \quad$ Glycerol

Add 1.8 $\mathrm{L}$ of $\mathrm{dH}_{2} \mathrm{O}$ and autoclave. Before use add $200 \mathrm{ml}$ Phosphate Buffer. 
Appendix $E$ (continued)

Table 7: 1 LE. coli Wash Buffer

$20 \mathrm{ml} \quad 1 \mathrm{M}$ Tris $\mathrm{pH}=8$

$10 \mathrm{ml} \quad 5 \mathrm{M} \mathrm{NaCl}$

Dilute to $1 \mathrm{~L}$ with $\mathrm{dH}_{2} \mathrm{O}$.

Table 8: 1 LPhosphate Buffered Saline (1 $\times$ PBS)

$8.0 \mathrm{~g} \quad \mathrm{NaCl}(137 \mathrm{mM})$

$0.2 \mathrm{~g} \quad \mathrm{KCl}(2.7 \mathrm{mM})$

$1.42 \mathrm{~g} \quad \mathrm{Na}_{2} \mathrm{HPO}_{4}(10 \mathrm{mM})$

$0.24 \mathrm{~g} \quad \mathrm{KH}_{2} \mathrm{PO}_{4}(2 \mathrm{mM})$

$187.5 \mathrm{~g}$ glycerol (15\%)

Add $800 \mathrm{ml} \mathrm{dH} 2 \mathrm{O}$ to the bottle and $\mathrm{pH}$ to 7.4 then fill to $1 \mathrm{~L}$. Autoclave or filter sterilize before use. 
APPENDIX F: NMR PEAKLIST FOR $\alpha \mathrm{c}(233-425)$

\begin{tabular}{|c|c|c|c|c|c|}
\hline assignment & ${ }^{15} \mathrm{~N} \mathrm{ppm}$ & ${ }^{1} \mathrm{H}$ ppm & assignment & ${ }^{15} \mathrm{~N} \mathrm{ppm}$ & ${ }^{1} \mathrm{H}$ ppm \\
\hline 1 & 112.288 & 7.488 & 41 & 122.081 & 8.541 \\
\hline 2 & 129.646 & 10.131 & 42 & 121.855 & 8.256 \\
\hline 3 & 129.613 & 10.099 & 43 & 121.761 & 8.393 \\
\hline 4 & 129.416 & 10.075 & 44 & 121.775 & 8.061 \\
\hline 5 & 129.358 & 10.147 & 45 & 121.583 & 8.226 \\
\hline 6 & 126.523 & 8.409 & 46 & 121.534 & 8.26 \\
\hline 7 & 125.876 & 8.3 & 47 & 121.481 & 8.337 \\
\hline 8 & 125.794 & 8.415 & 48 & 121.481 & 8.29 \\
\hline 9 & 125.606 & 8.387 & 49 & 121.333 & 8.438 \\
\hline 10 & 125.449 & 7.883 & 50 & 121.342 & 8.359 \\
\hline 11 & 124.919 & 8.207 & 51 & 121.318 & 7.916 \\
\hline 12 & 124.547 & 8.346 & 52 & 121.199 & 8.262 \\
\hline 13 & 124.393 & 8.177 & 53 & 121.146 & 8.554 \\
\hline 14 & 124.24 & 8.254 & 54 & 121.162 & 8.128 \\
\hline 15 & 124.107 & 8.292 & 55 & 121.097 & 8.5 \\
\hline 16 & 123.979 & 8.386 & 56 & 120.978 & 8.316 \\
\hline 17 & 123.884 & 8.416 & 57 & 120.813 & 8.15 \\
\hline 18 & 123.734 & 8.577 & 58 & 120.78 & 8.472 \\
\hline 19 & 123.634 & 8.253 & 59 & 120.731 & 8.52 \\
\hline 20 & 123.542 & 8.494 & 60 & 120.724 & 7.854 \\
\hline 21 & 123.342 & 8.164 & 61 & 120.724 & 7.844 \\
\hline 22 & 123.266 & 8.313 & 62 & 120.653 & 8.262 \\
\hline 23 & 123.229 & 8.437 & 63 & 120.49 & 8.443 \\
\hline 24 & 123.136 & 8.705 & 64 & 120.328 & 8.564 \\
\hline 25 & 123.073 & 8.402 & 65 & 119.935 & 8.095 \\
\hline 26 & 123.113 & 8.366 & 66 & 119.712 & 8.166 \\
\hline 27 & 122.928 & 8.721 & 67 & 119.491 & 8.036 \\
\hline 28 & 122.916 & 8.195 & 68 & 119.439 & 8.434 \\
\hline 29 & 122.867 & 8.237 & 69 & 119.189 & 8.263 \\
\hline 30 & 122.794 & 8.482 & 70 & 119.113 & 8.402 \\
\hline 31 & 122.801 & 8.271 & 71 & 118.949 & 8.366 \\
\hline 32 & 122.685 & 8.216 & 72 & 118.892 & 8.316 \\
\hline 33 & 122.661 & 8.443 & 73 & 118.813 & 8.403 \\
\hline 34 & 122.608 & 8.327 & 74 & 118.825 & 8.35 \\
\hline 35 & 122.566 & 8.6 & 75 & 118.781 & 8.29 \\
\hline 36 & 122.527 & 8.373 & 76 & 118.655 & 8.472 \\
\hline 37 & 122.358 & 8.298 & 77 & 118.56 & 8.501 \\
\hline 38 & 122.309 & 8.245 & 78 & 118.49 & 8.399 \\
\hline 39 & 122.262 & 8.577 & 79 & 118.085 & 8.223 \\
\hline 40 & 122.183 & 8.465 & 80 & 118.056 & 8.296 \\
\hline
\end{tabular}


APPENDIX F (continued)

$\begin{array}{cccccc}\text { assignment } & { }^{15} \mathbf{N ~ p p m} & { }^{1} \mathrm{H} \mathrm{ppm} & \text { assignment } & { }^{15} \mathrm{~N} \mathrm{ppm} & { }^{1} \mathrm{H} \mathrm{ppm} \\ 81 & 117.93 & 8.437 & 121 & 113.028 & 6.93 \\ 82 & 117.785 & 8.46 & 122 & 112.951 & 7.658 \\ 83 & 117.67 & 8.268 & 123 & 112.946 & 7.499 \\ 84 & 117.529 & 8.378 & 124 & 112.961 & 6.979 \\ 85 & 117.445 & 8.204 & 125 & 112.9 & 7.62 \\ 86 & 117.409 & 8.425 & 126 & 112.9 & 6.912 \\ 87 & 116.908 & 8.058 & 127 & 112.863 & 7.602 \\ 88 & 116.445 & 8.122 & 128 & 112.78 & 7.484 \\ 89 & 116.373 & 8.304 & 129 & 112.771 & 7.474 \\ 90 & 116.414 & 8.171 & 130 & 112.782 & 6.853 \\ 91 & 116.084 & 8.312 & 131 & 112.751 & 7.586 \\ 92 & 115.915 & 8.01 & 132 & 112.753 & 6.935 \\ 93 & 115.809 & 8.284 & 133 & 112.616 & 7.494 \\ 94 & 115.701 & 8.342 & 134 & 112.597 & 6.964 \\ 95 & 115.703 & 8.209 & 135 & 112.569 & 6.864 \\ 96 & 115.656 & 8.139 & 136 & 112.5 & 7.462 \\ 97 & 115.618 & 8.173 & 137 & 112.234 & 6.937 \\ 98 & 115.548 & 8.12 & 138 & 112.233 & 6.856 \\ 99 & 115.494 & 8.084 & 139 & 111.791 & 8.619 \\ 100 & 115.437 & 8.422 & 140 & 111.438 & 8.522 \\ 101 & 115.268 & 8.313 & 141 & 111.424 & 8.534 \\ 102 & 115.29 & 8.062 & 142 & 111.112 & 8.406 \\ 103 & 115.172 & 8.27 & 143 & 111.099 & 8.368 \\ 104 & 114.23 & 8.159 & 144 & 111.059 & 8.4 \\ 105 & 114.144 & 7.987 & 145 & 110.806 & 8.527 \\ 106 & 113.969 & 8.082 & 146 & 110.783 & 8.426 \\ 107 & 113.768 & 8.139 & 147 & 110.802 & 8.393 \\ 108 & 113.454 & 7.656 & 148 & 110.761 & 8.364 \\ 109 & 113.451 & 6.979 & 149 & 110.412 & 8.242 \\ 110 & 113.37 & 7.913 & 150 & 110.272 & 8.47 \\ 111 & 113.313 & 7.261 & 151 & 110.317 & 8.195 \\ 112 & 113.265 & 8.258 & 152 & 110.239 & 8.5 \\ 113 & 113.264 & 8.087 & 153 & 110.139 & 8.568 \\ 114 & 113.181 & 7.498 & 154 & 110.086 & 8.639 \\ 115 & 113.174 & 6.948 & 155 & 110.029 & 8.206 \\ 116 & 113.186 & 6.872 & 156 & 109.947 & 8.452 \\ 117 & 113.092 & 8.177 & 157 & 109.852 & 8.374 \\ 118 & 113.144 & 7.629 & 158 & 109.783 & 8.538 \\ & 113.129 & 6.961 & 109.709 & 8.596 \\ 19 & 112.983 & 7.592 & 109.625 & 8.523\end{array}$




\section{APPENDIX F (continued)}

$\begin{array}{ccc}\text { assignment } & { }^{15} \mathbf{N ~ p p m} & { }^{1} \mathbf{H} \text { ppm } \\ 161 & 108.948 & 8.506 \\ 162 & 108.918 & 8.472 \\ 163 & 108.909 & 8.334 \\ 164 & 108.823 & 8.358 \\ 165 & 108.815 & 8.446 \\ 166 & 107.949 & 8.267 \\ 167 & 107.658 & 8.205 \\ 168 & 132.122 & 8.078 \\ 169 & 119.788 & 8.254 \\ 170 & 113.171 & 7.322 \\ 171 & 112.27 & 7.589 \\ 172 & 129.965 & 10.091 \\ 173 & 110.822 & 8.569 \\ 174 & 110.805 & 8.669 \\ 175 & 110.651 & 8.637 \\ 176 & 115.748 & 8.46 \\ 177 & 115.782 & 8.262 \\ 178 & 121.697 & 8.432 \\ 179 & 121.084 & 8.644 \\ 180 & 121.578 & 8.608 \\ 181 & 122.942 & 8.325 \\ 182 & 113.242 & 7.19 \\ 183 & 113.396 & 7.694 \\ 184 & 129.669 & 10.078\end{array}$


APPENDIX G: NMR ASSIGNMENTS FOR $a C(233$ - 425) IN THE PRESENCE OF FXIII-A

\begin{tabular}{ccc} 
assignment & ${ }^{15} \mathbf{N ~ p p m}$ & ${ }^{1} \mathrm{H} \mathrm{ppm}$ \\
1 & 111.454 & 8.522 \\
2 & 129.976 & 10.092 \\
3 & 129.693 & 10.154 \\
4 & 129.664 & 10.13 \\
5 & 129.684 & 10.075 \\
6 & 129.628 & 10.097 \\
7 & 129.425 & 10.074 \\
8 & 129.368 & 10.146 \\
9 & 126.534 & 8.409 \\
10 & 125.884 & 8.299 \\
11 & 125.802 & 8.414 \\
12 & 125.615 & 8.387 \\
13 & 125.458 & 7.883 \\
14 & 124.927 & 8.208 \\
15 & 124.561 & 8.346 \\
16 & 124.404 & 8.177 \\
17 & 124.251 & 8.253 \\
18 & 124.122 & 8.292 \\
19 & 124.001 & 8.385 \\
20 & 123.902 & 8.416 \\
21 & 123.742 & 8.576 \\
22 & 123.644 & 8.254 \\
23 & 123.559 & 8.493 \\
24 & 123.359 & 8.163 \\
25 & 123.332 & 8.188 \\
26 & 123.236 & 8.437 \\
27 & 123.278 & 8.312 \\
28 & 123.149 & 8.706 \\
29 & 123.152 & 8.367 \\
30 & 123.089 & 8.4 \\
31 & 122.939 & 8.72 \\
32 & 122.931 & 8.324 \\
33 & 122.924 & 8.195 \\
34 & 122.796 & 8.481 \\
35 & 122.835 & 8.27 \\
36 & 122.703 & 8.358 \\
37 & 122.723 & 8.218 \\
38 & 122.667 & 8.442 \\
39 & 122.568 & 8.601 \\
\hline & & \\
\hline 3 & &
\end{tabular}

$\begin{array}{ccc}\text { assignment } & { }^{15} \mathbf{N ~ p p m} & { }^{1} \mathrm{H} \mathrm{ppm} \\ 40 & 122.615 & 8.327 \\ 41 & 122.546 & 8.373 \\ 42 & 122.37 & 8.297 \\ 43 & 122.344 & 8.35 \\ 44 & 122.319 & 8.246 \\ 45 & 122.204 & 8.57 \\ 46 & 122.168 & 8.465 \\ 47 & 122.105 & 8.54 \\ 48 & 121.869 & 8.258 \\ 49 & 121.807 & 8.359 \\ 50 & 121.792 & 8.061 \\ 51 & 121.737 & 8.429 \\ 52 & 121.777 & 8.394 \\ 53 & 121.583 & 8.23 \\ 54 & 121.558 & 8.612 \\ 55 & 121.541 & 8.26 \\ 56 & 121.499 & 8.339 \\ 57 & 121.496 & 8.29 \\ 58 & 121.427 & 8.554 \\ 59 & 121.362 & 8.359 \\ 60 & 121.347 & 8.438 \\ 61 & 121.327 & 7.915 \\ 62 & 121.214 & 8.264 \\ 63 & 121.156 & 8.554 \\ 64 & 121.17 & 8.131 \\ 65 & 121.089 & 8.645 \\ 66 & 121.107 & 8.5 \\ 67 & 120.99 & 8.316 \\ 68 & 120.822 & 8.149 \\ 69 & 120.751 & 8.52 \\ 70 & 120.789 & 8.472 \\ 71 & 120.69 & 8.677 \\ 72 & 120.732 & 7.853 \\ 73 & 120.73 & 7.843 \\ 74 & 120.665 & 8.261 \\ 75 & 120.503 & 8.444 \\ 76 & 120.339 & 8.564 \\ 77 & 119.946 & 8.096 \\ 78 & 119.806 & 8.255\end{array}$


APPENDIX G: (continued)

\begin{tabular}{|c|c|c|c|c|c|}
\hline assignment & ${ }^{15} \mathrm{~N} \mathrm{ppm}$ & ${ }^{1} \mathrm{H}$ ppm & assignment & ${ }^{15} \mathrm{~N} \mathrm{ppm}$ & ${ }^{1} \mathrm{H} \mathrm{ppm}$ \\
\hline 79 & 119.722 & 8.166 & 118 & 115.084 & 8.264 \\
\hline 80 & 119.521 & 8.034 & 119 & 114.245 & 8.16 \\
\hline 81 & 119.45 & 8.433 & 120 & 114.156 & 7.987 \\
\hline 82 & 119.254 & 8.308 & 121 & 113.987 & 8.083 \\
\hline 83 & 119.204 & 8.265 & 122 & 113.778 & 8.139 \\
\hline 84 & 119.13 & 8.402 & 123 & 113.461 & 7.656 \\
\hline 85 & 118.96 & 8.366 & 124 & 113.448 & 6.979 \\
\hline 86 & 118.905 & 8.316 & 125 & 113.383 & 7.913 \\
\hline 87 & 118.821 & 8.403 & 126 & 113.383 & 7.693 \\
\hline 88 & 118.834 & 8.349 & 127 & 113.329 & 7.261 \\
\hline 89 & 118.772 & 8.291 & 128 & 113.273 & 8.258 \\
\hline 90 & 118.672 & 8.471 & 129 & 113.276 & 8.088 \\
\hline 91 & 118.586 & 8.5 & 130 & 113.21 & 7.195 \\
\hline 92 & 118.479 & 8.398 & 131 & 113.153 & 7.628 \\
\hline 93 & 118.102 & 8.224 & 132 & 113.184 & 7.497 \\
\hline 94 & 118.068 & 8.296 & 133 & 113.188 & 7.339 \\
\hline 95 & 117.966 & 8.435 & 134 & 113.192 & 6.87 \\
\hline 96 & 117.815 & 8.459 & 135 & 113.093 & 8.177 \\
\hline 97 & 117.683 & 8.269 & 136 & 113.143 & 6.961 \\
\hline 98 & 117.543 & 8.377 & 137 & 113.054 & 6.929 \\
\hline 99 & 117.435 & 8.424 & 138 & 112.979 & 7.591 \\
\hline 100 & 117.445 & 8.205 & 139 & 112.962 & 7.658 \\
\hline 101 & 116.921 & 8.059 & 140 & 112.961 & 7.497 \\
\hline 102 & 116.429 & 8.171 & 141 & 112.95 & 6.978 \\
\hline 103 & 116.456 & 8.122 & 142 & 112.912 & 7.619 \\
\hline 104 & 116.386 & 8.303 & 143 & 112.87 & 7.601 \\
\hline 105 & 115.934 & 8.01 & 144 & 112.914 & 6.911 \\
\hline 106 & 115.822 & 8.284 & 145 & 112.853 & 7.643 \\
\hline 107 & 115.791 & 8.262 & 146 & 112.769 & 7.585 \\
\hline 108 & 115.729 & 8.462 & 147 & 112.792 & 7.483 \\
\hline 109 & 115.719 & 8.342 & 148 & $\$ 12.78$ & 7.471 \\
\hline 110 & 115.712 & 8.21 & 149 & 112.774 & 6.934 \\
\hline 111 & 115.641 & 8.138 & 150 & 112.793 & 6.853 \\
\hline 112 & 115.619 & 8.173 & 151 & 112.638 & 7.492 \\
\hline 113 & 115.548 & 8.12 & 152 & 112.617 & 6.964 \\
\hline 114 & 115.499 & 8.084 & 153 & 112.603 & 6.864 \\
\hline 115 & 115.452 & 8.422 & 154 & 112.513 & 7.46 \\
\hline 116 & 115.285 & 8.314 & 155 & 112.28 & 7.587 \\
\hline 117 & 115.295 & 8.061 & 156 & 112.263 & 7.486 \\
\hline
\end{tabular}


APPENDIX G: (continued)

$\begin{array}{ccc}\text { assignment } & { }^{15} \mathbf{N ~ p p m} & { }^{1} \mathrm{H} \mathrm{ppm} \\ 157 & 112.236 & 6.937 \\ 158 & 112.247 & 6.855 \\ 159 & 111.803 & 8.618 \\ 160 & 111.438 & 8.534 \\ 161 & 111.1 & 8.405 \\ 162 & 111.111 & 8.367 \\ 163 & 110.832 & 8.585 \\ 164 & 110.818 & 8.569 \\ 165 & 110.817 & 8.527 \\ 166 & 110.823 & 8.393 \\ 167 & 110.811 & 8.672 \\ 168 & 110.793 & 8.425 \\ 169 & 110.771 & 8.363 \\ 170 & 110.654 & 8.64 \\ 171 & 110.423 & 8.242 \\ 172 & 110.324 & 8.194 \\ 173 & 110.289 & 8.47 \\ 174 & 110.258 & 8.5 \\ 175 & 110.167 & 8.568 \\ 176 & 110.102 & 8.639 \\ 177 & 110.033 & 8.206 \\ 178 & 109.955 & 8.451 \\ 179 & 109.861 & 8.374 \\ 180 & 109.792 & 8.537 \\ 181 & 109.725 & 8.596 \\ 182 & 109.61 & 8.522 \\ 183 & 108.952 & 8.506 \\ 184 & 108.918 & 8.471 \\ 185 & 108.928 & 8.334 \\ 186 & 108.827 & 8.446 \\ 187 & 108.843 & 8.358 \\ 188 & 107.952 & 8.266 \\ 189 & 107.669 & 8.204 \\ 190 & 113.158 & 6.947 \\ 191 & 132.113 & 8.077\end{array}$


(Not defined in coagulation schematic and in alphabetical order)

1D- One Dimensional

2D- Two Dimensional

$a_{2}$ AP- $a_{2}$-antiplasmin

a-CHCA- a-cyano-4-hydroxycinnamic acid

AP- Activation Peptide

AUC- Analytical Ultracentrifugation

DMSO- Dimethylsulfoxide

DON- 6-diazo-5-oxo-norleucine

FXIIIalla - Thrombin Activated FXIII

FXIIIa ${ }^{\mathrm{Ca}}$ - Calcium Activated FXIII

GDH- Glutamate Dehydrogenase

GDP- Guanine Diphosphate

GEE- Glycine ethyl ester

HDX- Hydrogen Deuterium Exchange

HSQC- Heteronuclear Single Quantum Coherence

IAA- lodoacetamide

IC50- Concentration Resulting in 50\% Inhibition

IPAP- Inphase Antiphase

Kd- Dissociation Equilibrium Constant

MALDI- Matrix-Assisted Laser Desorption-Ionization

MS- Mass Spectrometry

MWCO- Molecular Weight Cut-Off

$\mathrm{m} / \mathrm{z}$ - Mass to Charge ratio

NMR- Nuclear Magnetic Resonance

$\mathrm{NADH}$ - Nicotinamide adenine dinucleotide

TGase- Transglutaminase

TG2- Transglutaminase 2

TOF- Time-of-Flight

TOCSY- Total Correlation Spectroscopy

TFA- Trifluoroacetic acid 
CURRICULUM VITAE

\author{
RICHARD T. WOOFTER II, PhD Candidate \\ 6083 Triple Crown Drive \\ Medina, $\mathrm{OH} 44256$ \\ Ricky.Woofter@gmail.com
}

\title{
EDUCATION:
}

PhD CHEMISTRY

Department of Chemistry

University of Louisville

Louisville, KY (December 2011)

BACHELOR OF SCIENCE DEGREE IN CHEMISTRY (ACS Certified):

College of Charleston

Charleston, SC (May 2001)

BACHELOR OF SCIENCE DEGREE IN BIOCHEMISTRY (ACS Certified):

College of Charleston

Charleston, SC (May 2000)

\section{RESEARCH EXPERIENCE:}

\section{GRADUATE RESEARCH:}

Department of Chemistry, University of Louisville, Louisville, KY

Utilized LC-ESI-MS for the analysis of volatile compounds released from A549 lung epithelial cells, 2006-2007.

\section{THESIS:}

Investigated Factor XIII structural dynamics during activation and inhibition utilizing MALDI-TOF-MS and NMR techniques, 2008-present

Utilized hydrogen-deuterium exchange (HDX) in conjunction with MALDITOF-MS to investigate Factor XIII conformational dynamics due to ionic concentration as well as nonproteolytically activation

Utilized HDX coupled with MALDI-TOF-MS to compare the conformational dynamics of FXIII-A $A_{2}$ to transglutaminase 2 when activated, unactivated and bound to substrate. 
Determine the residues on FXIII-A that are crucial for FXIII-A $\mathrm{A}_{2}$ heterotetramer formation using HDX technology. Also investigate the role of the fibrinogen aC-domain in the dissociation of FXIII-B.

Utilize ${ }^{1} \mathrm{H}^{15} \mathrm{~N}$ HSQC to probe whether the aC-domain contains any compact structure when interacting with recombinant C314A FXIII- $A_{2}$.

\section{GOVERNMENT:}

Marine Biotoxins Program, Center for Coastal Environmental Health and Biomolecular Research NOAA/NOS, Charleston, SC

Developed a radioimmunoassay (RIA) for the detection of brevetoxin, a potent neurotoxin produced by the marine algae Karenia brevis, in the blood of exposed animals, 2001-2002

Investigated possible therapeutic effects of cholestyramine in brevetoxin exposed mice, 2002-2003

Utilized the RIA to assist the Analytical Response Team in toxin analysis of tissue samples during marine mammal mortality events, 2002-2006

Monitored the uptake and elimination of brevetoxin in the blood of striped mullet (Mugil Cephalis), 2003-2004

Determined the distribution of brevetoxin within specific lipoprotein fractions of mouse blood and human plasma, 2003-2006

\section{UNDERGRADUATE RESEARCH:}

Department of Chemistry and Biochemistry, College of Charleston, Charleston, SC

Synthesized silyl-substituted organometallic fluorene compounds with $\mathrm{Dr}$. Jason Overby, Summer 2000

Department of Geology, College of Charleston, Charleston, SC

Observed the migrating and homing behavior of the chitin Acanthopleura granulata in San Salvador, Bahamas with Dr. James Carew, Spring 2001

Department of Physics and Astronomy, College of Charleston, Charleston, SC Studied the relationship between mass and velocity in Cub Scout pinewood derby cars with Dr. Laney Mills, Spring, 1999

\section{INDUSTRY:}

Charleston Technical Center, Westvaco, North Charleston, SC Assigned a project formulating new inks and toners as a co-op research analyst, 1999 and 2001 


\section{TEACHING EXPERIENCE:}

GEMS Science Fellow, Jefferson County Public Schools, Louisville, KY

Co-taught in a $7^{\text {th }}$ grade science classroom, Fall 2008-Spring 2010

Adjunct Faculty, Chemistry Department, Trident Technical College, North Charleston, SC

Chemistry 110 Lab and tutorial, Spring 2002, Spring 2003, and Fall 2004

\section{TEACHING ASSISTANT:}

Department of Chemistry, University of Louisville, Louisville, KY

Chemistry Recitation and Laboratory, 2007-2008

Department of Chemistry and Biochemistry, College of Charleston, Charleston, SC

Analytical and Organic Laboratory, 1998-2000

\section{AWARDS AND HONORS:}

- Groundwork Education in Mathematics and Science (GEMS) Fellow, National Science Foundation GK-12 Program, Partnership between University of Louisville and Jefferson County Public Schools, 2008-2010

- Departmental Fellow, University of Louisville Chemistry Department, 2006-2007

- Eagle Scout plus Bronze Palms, Troop 28 Spartanburg, SC

\section{ACADEMIC SERVICE AND PROFESSIONAL AFFILIATIONS:}

- Information Officer and founding member of the Arts and Sciences

Graduate Student Union, University of Louisville, 2010-2011

-Member, Protein Society, 2009-present

-President, Chemistry Graduate Student Association, University of Louisville, 2008-present

-Member, Gamma Delta Chapter of Alpha Chi Sigma, Professional Chemistry Fraternity, 1998-present

-Chair, Social Committee, National Ocean Service Marine Biotoxins Program, 2002-2006

-Member, International Society for the Study of Harmful Algae, 2002-2006

-Mentor, South Carolina Junior Academy of Science, Academic Magnet High School, 2001-2006

-Science Fair Judge, Charleston area schools, 2000-2006

-Master Alchemist, Gamma Delta Chapter of Alpha Chi Sigma, Chemistry Fraternity, 2000-2001

\section{PUBLICATIONS:}

Woofter, R., Maurer, M. Investigating the Importance of Calcium in the Conformational Dynamics of Factor XIII Activation by Utilizing Hydrogen/Deuterium Exchange in Conjunction with MALDI-TOF-MS. Arch Biochem Biophys. 2011 Aug 1;512(1):87-95. 
Woofter, R., Ramsdell, J. Distribution of Brevetoxin to Lipoproteins in human plasma. Toxicon, 2007. 1:49(7): p. 1010-8.

Woofter, R., Brendtro, K., Ramsdell, J. Uptake and Elimination of Brevetoxin in Blood of Striped Mullet (Mugil Cephalis) after Aqueous Exposure to Karenia Brevis, Environ Health Perspect, 2005. 113(1): p. $11-16$.

Woofter, R., Spiess, P., Ramsdell, J. Distribution of brevetoxin (PbTx-3) in mouse plasma: association with high-density lipoproteins. Environ Health Perspect. 2005. 113(11):1491-6.

Ramsdell, J., Woofter, R., Colman, J., Dechraou, MYB., Dover, S., Pandos, B., Gordon, C.J. Protective effects of cholestyramine on oral exposure to the red tide toxin brevetoxin, FASEB JOURNAL, 2003. 17 (4): A613-A613 Part 1 Suppl. S.

Woofter, R., Bottein Dechraoui, Y., Garthwaite, I., Towers, N., Gordon, C., Cordova, J, Ramsdell, J. Measurement of Brevetoxin Levels by Radioimmunoassay of Blood Collection Cards after Acute, Long-Term, and Low Dose Exposure in Mice. Environ Health Perspect, 2003. 111(13): p. 1595-1600.

Overby, J., Woofter, R., Rheingold, A., Incarvito, C., and Sommer, $\mathrm{R}$. The synthesis and structural characterization of silyl-substituted fluorenes. J Chem Crystallogr, 2003. 33(5-6): p. 357-364.

Woofter, R. Experiment XVI In: Organic Chemistry Laboratory Manual $2^{\text {nd }}$ Edition; An Introduction to Organic Laboratory: Techniques of Synthesis and Characterization, Part B $-2^{\text {nd }}$ Semester (Heldrich, Frederick J.); Wiley and Sons, Inc. 2000; p.69-71.

Woofter, R. Experiment XVII In: Organic Chemistry Laboratory Manual $2^{\text {nd }}$ Edition; An Introduction to Organic Laboratory: Techniques of Synthesis and Characterization, Synthesis of

Tetraphenylcyclopentadienone, Part B $-2^{\text {nd }}$ Semester (Heldrich, Frederick J.); Wiley and Sons, Inc. 2000; p. 81-84.

\section{PRESENTATIONS:}

Invited Talks:

Woofter, R., Sexton, B., Rich, C. Developing and Implementing Meaningful NSF GK-12 Partnerships: Some Do's and Don'ts. American Association for the Advancement of Science/National Science Foundation Annual GK-12 Conference, Washington D C, March, 26-28, 2010. 


\section{Posters:}

Woofter, R., Sexton, B., Rich, C. GEMS Fellows and Teachers Focus on Strategies for Informed Science Instruction: Formative Assessment Activities that Complement Inquiry-Based Curricula. American Association for the Advancement of Science/National Science Foundation Annual GK-12 Conference, Washington D C, March, 26-28, 2010.

Woofter, R., Sabo, M., Maurer, M. Investigating Factor XIII Structural Changes in the Presence of Mono and Divalent Cations Utilizing HDX coupled with MALDI-TOF-MS. $23^{\text {rd }}$ Symposium of the Protein Society, Boston, MA. July 25-29, 2009.

Ramsdell, J., Woofter, R., Brendtro, K. Uptake and Elimination of Brevetoxin in Blood of Striped Mullet (Mugil Cephalis) after Aqueous Exposure to Karenia Brevis. $11^{\text {th }}$ International Conference on Harmful Algae, Cape Town, South Africa. November 14-19, 2004.

Spiess, P., Woofter, R., Ramsdell, J. The Distribution of Brevetoxin (PbTx-3) to Specific Lipoprotein Fractions in Mouse Blood. Second Symposium on Harmful Marine Algae in the U.S., Woods Hole, MA. December 9-13, 2003.

Woofter, R., Brendtro, K., Miles, C., Towers, N., Ramsdell, J. Biomonitoring Blood Brevetoxin in Striped Mullet (Mugil cephalis) after Sub-lethal Laboratory Exposure to Karenia Brevis. Second Symposium on Harmful Marine Algae in the U.S., Woods Hole, MA. December 9-13, 2003.

Ramsdell, J., Woofter, R., Colman, J., Bottein Dechraoui, M-Y, Dover, S., Pandos, B., Gordon, C. Protective effects of cholestyramine on oral exposure to the red tide toxin brevetoxin. Experimental Biology 2003 Translating the Genome, San Diego, CA, April, 11-15, 2003.

Woofter, R., Bottein Dechraoui, M-Y., Garthwaite, I., Towers, N., Gordon, C., Cordova, J, Ramsdell, J. Comparison of Receptor and Immunoassays for Brevetoxin Detection in the Blood of Exposed Animals. $X^{\text {th }}$ International Conference on Harmful Algea, St. Pete Beach, FL. October, 21-25, 2002. 